\title{
Computational Methods For \\ Light Scattering By Metallic NANOPARTICLES
}

\author{
BY \\ Walter Ross Campbell Somerville \\ A thesis \\ submitted to Victoria University of Wellington \\ in fulfilment of the requirements for the degree of \\ Doctor of Philosophy
}

Victoria University of Wellington 2014 



\section{Abstract}

The unifying theme of this thesis is that of light scattering by particles, using computational approaches. This contributions here are separated into two main areas. The first consists of examining the behaviour of the extended boundary-condition method and $T$-matrix method, and providing a modified set of equations to use to calculate the relevant integrals. From this, some linear relations between integrals were found, which hint at the possibility of a more efficient means of performing these calculations. As well as this, the severe numerical problems associated with this method were investigated, and the primary source of these problems was identified in the case of two commonly-used shapes, spheroids and offset spheres. The cause of these numerical problems is that dominant, leading terms in the power series expansion of the integrands integrate identically to zero, but in practice, numerical calculations have insufficient precision to compute this exactly, and the overwhelming errors from this lead to drastically incorrect results. Following this identification, a new formulation of the integrals for spheroids is presented, which allows the much easier treatment of spheroids, approaching the level of ease of calculations for spheres in Mie theory. This formulation replaces some terms in the integrands with modified terms, that do not contain the parts of the power series that cause problems. As these should integrate to zero, we are able to remove them from the integrand without affecting the correct result.

The second area of this thesis is concerned with calculations of the near-field for systems of interest in plasmonics, and specifically in surface-enhanced Raman spectroscopy. Here, the enhancement of the electric field in the vicinity of a metallic surface has a large effect on measured signals. The contribution of this thesis is to study the geometric parameters that influence the distribution of the field enhancement at the particle's resonance, specifically focusing on different effects caused 
by the overall shape of the particle, as opposed to those effects due to the local shape of the particle in regions of high enhancement. It is shown that the overall shape determines the location of the resonance, while the local shape determines how strongly the enhancement is localised. Understanding the factors that influence the enhancement localisation will help in guiding the design of suitable plasmonic substrates. 


\section{Acknowledgements}

As I sit here, approaching rapidly the end, I owe thanks to many people who ensured that I had such a fun and productive time over the last three years. Primarily, I would like to thank my supervisor, Eric Le Ru, for making this $\mathrm{PhD}$ interesting and fun, through his rigorous-but-laid-back attitude to science (and life), as evidenced by the many trips to plunge into the less-than-tropical Wellington Harbour.

Secondly, I would like to acknowledge Pablo Etchegoin, initially my secondary supervisor, who did not live to see the end of this thesis. However Pablo provided much inspiration, and his willingness to discuss science, or any other topic, made the Raman Lab at VUW an amazing place to be part of, and I hope this atmosphere continues. I feel one of his quotes is appropriate here, "No one ever finishes their thesis, they abandon it."

All of the other group members have made working here fun. Baptiste Auguié, through assisting with my research, making cakes and generally fomenting mischief is responsible for a lot. A fond tradition of the group, driven by myself and Baptiste, was frequent cakes. For the reader's culinary pleasure, there are a collection of tested recipes beginning each chapter. Camille Artur has been a great neighbour. The other group members, Stefan, Ben, Brendan, Peter, James, Harry, Olly, Rene, Marina, Luke, Sam, Antoine, Lina, Rowan, Srikanth, Dmitri, Felix, Stanley and Chris also all embraced and enhanced the spirit of the Raman Lab. And of course Joe Trodahl, who comes in every morning hoping one of his students is there, but usually having to settle for some inane conversation with me.

One of the small things to stop us from taking science too seriously was the filming of the as-yet unrecognised masterpiece Mini Shark vs Tiny Octopus, which was a true collaborative effort. Thanks to all the other participants, Shrividya, the two Bens, Martin, Camille and Natalie. Thank you Matthias Meyer, both for many interesting discussions while you were still in Wellington, and helping me with blender from far across the sea.

To Alex Bouhelier and Eric Finot, and the rest of the ICB, thank you for hosting me in Dijon. 
I thank the Royal Society of New Zealand for providing funding through a Marsden Grant. I also thank the MacDiarmid Institute and MESA for the support they have provided.

To the various SCPS staff who provided assistance, I am grateful. Especially I would like to thank Dan for his always entertaining attitude and Ben and Krista for encouraging some crazy running adventures, Natasha for many lunchtime conversations, Christina for reading some of (and then leaving on a bus) this thesis, Eva for offering some wisdom regarding thesis-writing, and Andrea for many weekends shared writing our theses. I also thank Petrik, for assuming the role of secondary supervisor, and providing feedback on this thesis.

I thank all of my friends, especially Peter and Ben for many the times spent watching sport or playing pool throughout our undergraduate and postgraduate studies.

Lastly, I would like to thank my parents, for their unceasing support during all of my studies. They must be happy all of us children have now concluded our studies, even if I took much longer than my siblings. 


\section{Contents}

Abstract i

Acknowledgements iii

Contents v v

\begin{tabular}{|l|l}
\hline List of Figures & ix
\end{tabular}

Glossary

List of Symbols $\quad$ xiii

1 Introduction 1

1.1 Motivation and Goals . . . . . . . . . . . . . . . . . . . 1

1.2 Outline . . . . . . . . . . . . . . . . . . . . . . 2

2 Light Scattering 5

2.1 Applications . . . . . . . . . . . . . . . . . . . 5

2.2 General Light Scattering . . . . . . . . . . . . . . . . . . . . 6

2.2 .1 Field Expressions . . . . . . . . . . . . . . . . 6

2.2 .2 Maxwell's Equations . . . . . . . . . . . . . . . . 7

2.2 .3 Plane Waves . . . . . . . . . . . . . . . . . . . . . . . 11

2.2 .4 Boundary Conditions . . . . . . . . . . . . . . . . . . 12

2.2 .5 Scattering Quantities . . . . . . . . . . . . . . . 13

2.2 .6 Far-Field Measurements . . . . . . . . . . . . . . . . . 16

2.3 Surface-Enhanced Raman Spectroscopy . . . . . . . . . . . . . . . . . 18

2.3 .1 Raman Scattering . . . . . . . . . . . . . . . . . . 19 
Contents

$2.3 .2 \quad$ Surface-Enhanced Raman Spectroscopy . . . . . . . . . . . . . 20

2.4 Computational Techniques . . . . . . . . . . . . . . . . 22

2.4 .1 Mie Theory . . . . . . . . . . . . . . . . . . 23

$2.4 .2 \quad$ T-matrix Method . . . . . . . . . . . . . . . . . . . 24

$2.4 .3 \quad$ Finite-Element Method . . . . . . . . . . . . . . . . . . . . . 25

2.4 .4 Other Methods . . . . . . . . . . . . . . . . . . 26

3 Mie Theory and the T-matrix Method 29

3.1 Helmholtz Equation in Spherical Coordinates . . . . . . . . . . . . . 29

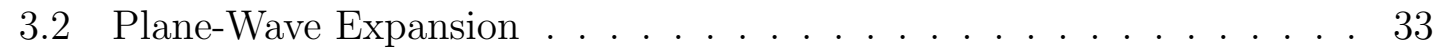

3.3 Mie Theory . . . . . . . . . . . . . . . . . . . . . . . . . . 36

3.3.1 Boundary Conditions . . . . . . . . . . . . . . . . . . . 37

3.3 .2 Scattering Quantities . . . . . . . . . . . . . . . . . 38

$3.4 \quad$ T-matrix Principles . . . . . . . . . . . . . . . . . . . . . 38

$3.4 .1 \quad$ Implementation Details . . . . . . . . . . . . . . . . . 41

3.4 .2 Axisymmetric Particles . . . . . . . . . . . . . . . . . . . . . 43

3.4 .3 Surface Fields . . . . . . . . . . . . . . . . . . . . . . . . . . . 44

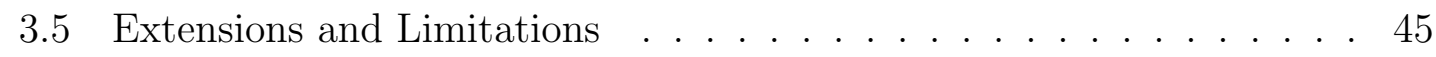

3.5.1 Multiple Scattering . . . . . . . . . . . . . . . . . . . . 45

3.5 .2 Layered Scattering . . . . . . . . . . . . . . . . . 46

3.5 .3 Numerical Problems . . . . . . . . . . . . . . . . . . . . . . . 46

3.5.4 Fundamental Problems . . . . . . . . . . . . . . . . . . . . 47

3.6 Overview of this Work . . . . . . . . . . . . . . . . . . . . . . . . . . . . . 48

$4 \quad T$-matrix Formalism and the Extended Boundary-Condition Method 51

4.1 New Integral Expressions . . . . . . . . . . . . . . . . . . . . 51

$4.1 .1 \quad$ Simplified Expressions $\ldots \ldots \ldots$. . . . . . . . . . . . 52

4.1 .2 Useful Relations . . . . . . . . . . . . . . . . . . . . . . . . . 54

4.1 .3 Derivation of $\mathbf{U}^{12}$ and $\mathbf{U}^{21}$. . . . . . . . . . . . . . 55

4.1 .4 Derivation of $\mathbf{U}^{11}$ and $\mathbf{U}^{22}$. . . . . . . . . . . . . . . . 58

$4.1 .5 \quad$ Benefits of the New Expressions . . . . . . . . . . . . . . . . 62

$4.1 .6 \quad$ Summary of which Expressions are Used . . . . . . . . . . . . 62 
$4.2 \quad T$-matrix Recursion Relations . . . . . . . . . . . . . . . . . 63

4.2 .1 Finding Relations . . . . . . . . . . . . . . . . . 64

4.2 .2 Obtaining Exact Relations . . . . . . . . . . . . . . 66

4.2 .3 Using the Relations . . . . . . . . . . . . . . . . . . 79

4.3 Summary $\ldots \ldots \ldots \ldots \ldots$

5 Problematic Spheroids 183

5.1 Geometries . . . . . . . . . . . . . . . . . . 84

5.1 .1 Spheroids . . . . . . . . . . . . . . . . . 84

5.1 .2 Offset Sphere . . . . . . . . . . . . . . . . . . . 86

5.1 .3 Cylinder . . . . . . . . . . . . . . . . 87

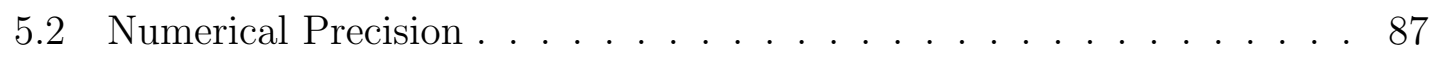

5.3 The Problem with Spheroids . . . . . . . . . . . . . . . . . . . . . . 89

5.4 Proof that Spheroid Integrals Contain Terms Integrating to Zero . . . 96

$5.4 .1 \quad$ Necessary Relations . . . . . . . . . . . . . . . . . . 96

$5.4 .2 \quad$ Integrals $\mathbf{L}^{1}$ and $\mathbf{L}^{2} \ldots \ldots \ldots \ldots$. . . . . . . . . . . 98

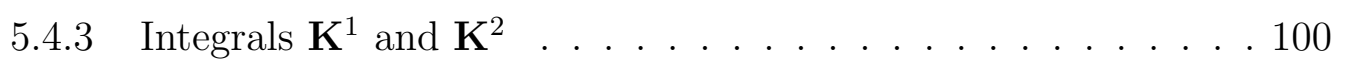

5.4 .4 Integrals $\mathbf{L}^{3}$ and $\mathbf{L}^{4} \ldots \ldots \ldots 10 . \ldots \ldots$

5.5 Inversion of the Correct Matrices . . . . . . . . . . . . . . . . . . 105

5.6 Spheroid Integral Forms . . . . . . . . . . . . . . . . . . . . . . . 109

5.6 .1 Basic Idea . . . . . . . . . . . . . . . . . . . . 110

5.6 .2 Series Method . . . . . . . . . . . . . . . . . 111

5.6 .3 Recursion to Fill the Matrix . . . . . . . . . . . . . . . . . . . 118

5.6 .4 Derivatives . . . . . . . . . . . . . . . . . 120

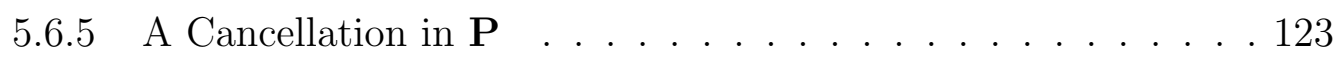

5.6 .6 Demonstration of Improved Performance . . . . . . . . . . . . . 124

5.7 Discussion and Outlook . . . . . . . . . . . . . . . . . . . 131

6 Application of Electromagnetic Calculations to Plasmonics 133

6.1 Bipyramids and Bicones . . . . . . . . . . . . . . . . 134

6.1 .1 Geometry . . . . . . . . . . . . . . . . 135

6.1 .2 Simulation Techniques . . . . . . . . . . . . . . 137

6.1 .3 Results . . . . . . . . . . . . . . . . . . . . . . . . . . . 139 
$6.2 \quad$ Chains of Spheres . . . . . . . . . . . . . . . . . . . . . . . . 142

6.3 EF Localisation . . . . . . . . . . . . . . . . . . . . . . . . . . . . . . 144

6.3 .1 Different Metrics . . . . . . . . . . . . . . . . . . 146

6.3 .2 Shape Effect . . . . . . . . . . . . . . . . . . . . . . . . . . 148

6.3 .3 Effect of Different Parameters . . . . . . . . . . . . . . . 152

6.3 .4 Implications of Different Metrics. . . . . . . . . . . . . . . . . 159

\begin{tabular}{lll}
\hline 7 & Discussion & 161
\end{tabular}

\begin{tabular}{ll}
\hline Appendices & 165
\end{tabular}

\begin{tabular}{|ll|}
\hline A Bessel Function Series Expressions & 165
\end{tabular}

\begin{tabular}{|l|l|}
\hline B Angular Function Definitions and Relations & 167
\end{tabular}

B.1 Function Definitions . . . . . . . . . . . . . . . . . . . . . . 167

B.2 Numerical Computation of the Functions . . . . . . . . . . . . . . . . 169

B.3 Relations Used in Proofs . . . . . . . . . . . . . . . . . . . . . . . . . 170

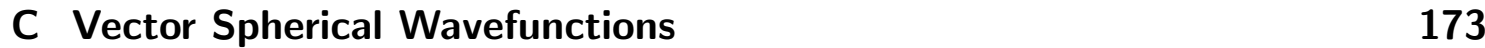

\begin{tabular}{ll|l|l|l|l}
\hline D & $J$-integral Expressions & 175
\end{tabular}

E Proof that Leading Terms Make No Contribution in Offset-Sphere Inte$\begin{array}{lr}\text { grals } & 177\end{array}$

E.1 Some Definitions . . . . . . . . . . . . . . . . . . . . . . 177

E.2 Proofs . . . . . . . . . . . . . . . . . . . . . . . . 181

E.2.1 Integrals $\mathbf{K}^{1}$ and $\mathbf{K}^{2}$. . . . . . . . . . . . . . . . . . . . . . . 181

E.2.2 $\quad$ Integrals $\mathbf{L}^{1}$ and $\mathbf{L}^{2}$. . . . . . . . . . . . . . . . . . . . . . . 182

E.2.3 Integrals $\mathbf{L}^{3}$ and $\mathbf{L}^{4} \ldots \ldots \ldots \ldots$. . . . . . . . . . . . . . . . . . . . . . . . . .

F Demonstration of Problems Near $s=1 \quad 187$

\begin{tabular}{ll}
\hline References & 191
\end{tabular}

viii 


\section{List of Figures}

2.1 Plane wave incident on a scatterer . . . . . . . . . . . . . . . . . 12

$2.2 \quad$ Schematic of extinction measurement . . . . . . . . . . . . . . . . 17

2.3 Angular-dependent scattering experiment . . . . . . . . . . . . . . . . 18

$2.4 \quad$ Dark-field microscopy schematic . . . . . . . . . . . . . . . . . . . . . 19

4.1 Depiction of Special Case 2

4.2 Depiction of General Case 1 . . . . . . . . . . . . . . . . . . . . 73

4.3 Depiction of General Case 2 . . . . . . . . . . . . . . . . . . . . 80

5.1 Convergence behaviour of scattering cross section . . . . . . . . . . . 91

5.2 Integrand and integral for offset sphere, spheroid and cylinder . . . . 93

5.3 Integral scaling behaviour . . . . . . . . . . . . . . . 95

5.4 Numerical performance of double-precision inversion to obtain T . . . 107

5.5 Numerical problems in the naïve series implementation for $F_{n k}^{+}$. . . . 113

$5.6 \quad$ Improved numerical performance for new series implementation for $F_{n k}^{+} 117$

5.7 Comparison of error accumulated by different recursion schemes . . . 121

5.8 Agreement of $\mathbf{P}$ and $\mathbf{Q}$ using old MATLAB code, which does not take cancellations into account . . . . . . . . . . . . . . . . . . 125

5.9 Agreement of $\mathbf{P}$ and $\mathbf{Q}$ using new MATLAB code . . . . . . . . . . 126

5.10 Numerical performance of new double-precision inversion to obtain T 128

5.11 The size of the converged sub-matrix of $\mathbf{T}$ as the size of $\mathbf{P}$ and $\mathbf{Q}$ is varied, for different values of required relative precision $\alpha$. The maximum order for the upper- and lower-triangular parts of $\mathbf{T}$ are shown, but by symmetry the lower triangle may be obtained from the upper triangle. . . . . . . . . . . . . . . . . . . . . . 129

5.12 Time taken for different implementations to calculate $\mathbf{P}$ and $\mathbf{Q}$. . . . 130 
6.1 TEM image of a bipyramid . . . . . . . . . . . . . . . . . . . . . . . 134

6.2 Schematic of a bicone as modelled in this thesis . . . . . . . . . . . 136

6.3 Overlay of bicone geometry onto TEM image . . . . . . . . . . . . . . 137

6.4 Angular dependence of $F$ on the surface of a bicone, accounting for orientation-averaging . . . . . . . . . . . . . . . . . . . . 140

6.5 SERS enhancement factor near a bicone . . . . . . . . . . . . . . . . 141

6.6 Comparison of simulation results from COMSOL and generalised Mie theory for a silver dimer . . . . . . . . . . . . . . . . . . . . . . . . 143

6.7 Extinction spectra of chains of spheres, as simulated by COMSOL . . 145

6.8 A selection of spheroids . . . . . . . . . . . . . . . . . . . . . . . . 149

6.9 A selection of bicones of varying parameters . . . . . . . . . . . . . . 150

6.10 A collection of modified spheroids . . . . . . . . . . . . . . . 151

6.11 Variation of properties of spheroids of different size and material . . . 153

6.12 Variation of properties of bicones of different shapes . . . . . . . . . . 154

6.13 Difference in local $F$ near bicones of different tip curvatures . . . . . 155

6.14 Various properties of different modified spheroids . . . . . . . . . . . 157

6.15 Various properties of different modified spheroids having the same resonance . . . . . . . . . . . . . . . . . 158 


\section{Glossary}

$\begin{array}{ll}\text { T-matrix } & \text { Transition matrix. } \\ \text { 2D } & \text { 2-dimensional. } \\ \text { 3D } & \text { 3-dimensional. } \\ \text { CTAB } & \text { Cetyltrimethylammonium bromide. } \\ \text { Cup } & \text { 250 ml. } \\ \text { DDA } & \text { Discrete-dipole approximation. } \\ \text { DNA } & \text { Deoxyribonucleic acid. } \\ \text { EBCM } & \text { Extended boundary-condition method. } \\ \text { EF } & \text { Enhancement factor. } \\ \text { FEM } & \text { Finite-element method. } \\ \text { GMP } & \text { GNU Multiple Precision Arithmetic Library. } \\ \text { GNU } & \text { GNU's Not Unix. } \\ \text { LU } & \text { Lower Upper. } \\ \text { PML } & \text { Perfectly matched layer. } \\ \text { RAM } & \text { Random-access memory. } \\ \text { SERS } & \text { Surface-enhanced Raman spectroscopy. } \\ \text { SI } & \text { International System of Units. } \\ \text { SIE } & \text { Surface-integral equation. } \\ \text { SM-SERS } & \text { Single-molecule surface-enhanced Raman spec- } \\ \text { Tbsp } & \text { troscopy. } \\ \text { TEM } & \text { Tablespoon, } 15 \text { ml. } \\ \text { tsp } & \text { Teaspoon, 5 ml. } \\ \text { VSWF } & \text { Vector spherical wavefunction. } \\ & \end{array}$





\section{List of Symbols Used in this Thesis}

$\begin{array}{ll}A & \text { A value being compared (assumed less accurate) } \\ A & \text { Collection of various terms for recursion relation } \\ A^{\prime} & \text { Collection of various terms for recursion relation } \\ B & \text { A value being compared (assumed more accurate) } \\ B & \text { Collection of various terms for recursion relation } \\ B^{\prime} & \text { Collection of various terms for recursion relation } \\ \vec{B} & \text { Magnetic induction vector (real) } \\ \vec{B}_{n m}(\theta) & \text { Vector for generating VSWF } \\ \vec{B}_{n m}(\theta, \phi) & \text { Vector for generating VSWF } \\ \vec{B} & \text { Magnetic induction vector (complex) } \\ \vec{C}_{n m}(\theta) & \text { Vector for generating VSWF } \\ \vec{C}_{n m}(\theta, \phi) & \text { Vector for generating VSWF } \\ C_{n} & \text { Prefactor for associated Legendre function, with } \\ & \text { implicit degree } m, \text { including the Condon-Shortley } \\ D & \text { phase. } \\ D & \text { Offset amount for offset sphere } \\ \vec{D} & \text { Semiaxis length of spheroid for bipyramid (along } \\ \vec{D} & \vec{z}) \\ \vec{D}_{1} & \text { Electric displacement vector (real) } \\ \vec{D}_{2} & \text { Electric displacement vector (complex) } \\ & \text { Electric displacement field vector outside particle } \\ & \text { (complex) } \\ & \text { Electric displacement field vector inside particle } \\ & \text { (complex) }\end{array}$


List of Symbols

E

$\vec{E}$

$\vec{E}$

$\vec{E}_{\mathrm{Ext}}$

$\vec{E}_{\text {Inc }}$

$\vec{E}_{\mathrm{Loc}}$

$\vec{E}_{\mathrm{L}}$

$\vec{E}_{\mathrm{R}}$

$\vec{E}_{\mathrm{Sca}}$

$\vec{E}_{+}$

$\overrightarrow{E_{-}}$

$\vec{E}_{0}$

$\vec{E}_{633}$

$\vec{E}_{705}$

$\vec{E}$

$\underline{\vec{E}}_{0}$

$\vec{E}_{1}$

$\vec{E}_{2}$

$E_{0}$

$E_{\phi+}$

$E_{\phi-}$

$E_{\theta+}$

$E_{\theta-}$

$E_{r+}$

$E_{r-}$

$\mathcal{E}$

$\mathrm{EF}$

$\mathrm{EF}_{\text {max }}$

$\langle\mathrm{EF}\rangle$

F
Semiaxis length of spheroid for bipyramid (along $\vec{x}, \vec{y})$

Electric field vector (real)

Electric field vector (complex)

External electric field vector (complex)

Incident electric field vector (complex)

Local electric field vector

Electric field vector at incident wavelength (complex)

Electric field vector at Raman wavelength (complex)

Scattered electric field vector (complex)

Electric field vector on outside of surface (complex)

Electric field vector on inside of surface (complex)

Incident electric field magnitude vector (complex)

Electric field vector at $\lambda=633 \mathrm{~nm}$ (complex)

Electric field vector at $\lambda=705 \mathrm{~nm}$ (complex)

Electric field vector (complex)

Complex electric field amplitude vector

Electric field vector outside particle (complex)

Electric field vector inside particle (complex)

Incident electric field magnitude (complex)

$\phi$-component of electric field outside surface

$\phi$-component of electric field inside surface

$\theta$-component of electric field outside surface

$\theta$-component of electric field inside surface

$r$-component of electric field outside surface

$r$-component of electric field inside surface

Operator returning even part of function

Enhancement factor

Maximum enhancement factor

Enhancement factor averaged over entire surface

SERS enhancement factor 
$\langle F\rangle$

$\langle F\rangle$

$F^{+}$

$F_{n k}$

$\vec{H}$

$\vec{H}_{\text {Inc }}$

$\vec{H}_{\text {Int }}$

$\vec{H}_{\mathrm{Sca}}$

$\underline{\vec{H}}$

$\underline{\vec{H}}_{0}$

$\vec{H}_{1}$

$\vec{H}_{2}$

J

$\mathbf{J}^{11}, \mathbf{J}^{12}, \mathbf{J}^{21}, \mathbf{J}^{22}$

$\vec{J}$

$\vec{J}_{f}$

$\underline{\vec{J}}_{f}$

$\hat{K}_{n k}^{1}$

$\hat{K}_{n k}^{2}$

$\mathbf{K}^{1}$

$\mathbf{K}^{2}$

$K_{n k p}^{1}$

$K_{n k p}^{2}$

$\hat{L}_{n k}^{1}$

$\hat{L}_{n k}^{2}$

$\mathbf{L}^{1}$

$\mathbf{L}^{2}$

$\mathbf{L}^{3}$

$\mathbf{L}^{4}$

$\mathbf{L}^{5}$

$\mathbf{L}^{6}$
Average value of $F$ over all incident orientations Average value of $F$ over entire surface

The terms with non-negative exponent of $F$

A function containing Riccati-Bessel functions

Magnetic field vector (real)

Incident magnetic field vector (complex)

Internal magnetic field vector (complex)

Scattered magnetic field vector (complex)

Magnetic field vector (complex)

Complex magnetic field amplitude vector

Magnetic field vector outside particle (complex)

Magnetic field vector inside particle (complex)

The $J$-matrix

The blocks of the $J$-matrix

Current density (real)

Free current density (real)

Free current density (complex)

The integrand of $K_{n k}^{1}$, excluding the prefactor $a_{n} a_{k}$

The integrand of $K_{n k}^{2}$, excluding the prefactor $a_{n} a_{k}$

Matrix of integrals

Matrix of integrals

Element from power-series expansion with exponent $p$

Element from power-series expansion with exponent $p$

The integrand of $L_{n k}^{1}$, excluding the prefactor $a_{n} a_{k}$

The integrand of $L_{n k}^{2}$, excluding the prefactor $a_{n} a_{k}$

Matrix of integrals

Matrix of integrals

Matrix of integrals

Matrix of integrals

Matrix of integrals

Matrix of integrals 
List of Symbols

\begin{tabular}{|c|c|}
\hline $\mathbf{L}^{7}$ & Matrix of integrals \\
\hline $\mathbf{L}^{8}$ & Matrix of integrals \\
\hline $\mathbf{L}^{31}$ & Matrix of integrals \\
\hline $\mathbf{L}^{32}$ & Matrix of integrals \\
\hline$\tilde{L}_{n}^{1}$ & Vector of integrals \\
\hline$\tilde{L}_{n}^{2}$ & Vector of integrals \\
\hline$\tilde{L}_{n}^{3}$ & Vector of integrals \\
\hline$L_{n k p}^{1}$ & $\begin{array}{l}\text { Element from power-series expansion with expo- } \\
\text { nent } p\end{array}$ \\
\hline$L_{n k p}^{3}$ & $\begin{array}{l}\text { Element from power-series expansion with expo- } \\
\text { nent } p\end{array}$ \\
\hline$L_{n k p}^{31}$ & $\begin{array}{l}\text { Element from power-series expansion with expo- } \\
\text { nent } p\end{array}$ \\
\hline$L_{n k p}^{32}$ & $\begin{array}{l}\text { Element from power-series expansion with expo- } \\
\text { nent } p\end{array}$ \\
\hline$M_{\text {Loc }}$ & Local electric field intensity enhancement factor \\
\hline$\vec{M}$ & Magnetisation (real) \\
\hline$\vec{M}$ & Magnetic VSWF \\
\hline$\vec{M}^{(1)}$ & Regular magnetic VSWF \\
\hline$\vec{M}^{(3)}$ & Irregular magnetic VSWF \\
\hline$\langle M\rangle$ & Average value of $M$ over entire surface \\
\hline$N$ & Maximum multipole order \\
\hline$\vec{N}$ & Electric VSWF \\
\hline$\vec{N}^{(1)}$ & Regular electric VSWF \\
\hline$\vec{N}^{(3)}$ & Irregular electric VSWF \\
\hline$P(i)$ & Polynomial in $i$ \\
\hline $\mathcal{P}^{+}$ & $\begin{array}{l}\text { Operator returning terms in power series with non- } \\
\text { negative power }\end{array}$ \\
\hline $\mathcal{P}^{-}$ & $\begin{array}{l}\text { Operator returning terms in power series with neg- } \\
\text { ative power }\end{array}$ \\
\hline $\mathcal{P}_{N}(\cos \theta)$ & Polynomial of degree up to $N$ in $\cos \theta$ \\
\hline $\mathbf{P}$ & $\begin{array}{l}\text { The } P \text {-matrix, relating scattered and internal field } \\
\text { coefficients }\end{array}$ \\
\hline
\end{tabular}




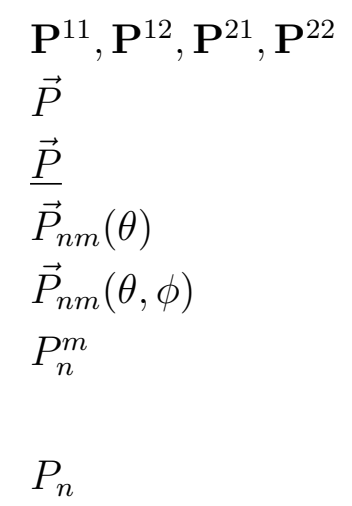

Q

$\mathrm{Q}^{11}, \mathrm{Q}^{12}, \mathrm{Q}^{21}, \mathrm{Q}^{22}$

$Q_{\text {Abs }}$

$Q_{\text {Ext }}$

$Q_{\text {Sca }}$

$R$

$R(r)$

$\mathbf{R}$

$R_{\text {experiment }}$
$R_{\text {simulation }}$

$R_{\text {tip only }}$

$\operatorname{Rg} \mathbf{J}$

$\operatorname{Rg} \mathbf{J}^{11}, \operatorname{Rg} \mathbf{J}^{12}$,

$\operatorname{Rg} \mathbf{J}^{21}, \operatorname{Rg} \mathbf{J}^{22}$

$S$

$S_{\text {Inc }}$

$\vec{S}$

$\vec{S}_{\text {Sca }}$

$\mathrm{T}$
The blocks of the $P$-matrix

Polarisation density vector (real)

Polarisation density vector (complex)

Vector for generating VSWF

Vector for generating VSWF

Associated Legendre function of degree $m$ and order $n$

Associated Legendre function, with implicit degree $m$ and argument $\cos \theta$

The $Q$-matrix, relating incident and internal field coefficients

The blocks of the $Q$-matrix

Absorption efficiency

Extinction efficiency

Scattering efficiency

Ratio of maximum to average enhancement factors

Radial part of VSWF

Matrix relating incident and internal field coefficients

Experimentally measured value of $R$

Value of $R$ from simulation, over entire particle surface

Value of $R$ from simulation considering only the tip

The regular part of the $J$-matrix

The blocks of the regular part of the $J$-matrix

Surface area

Incident power density

Time-averaged Poynting vector (real)

Time-averaged scattering Poynting vector (real)

The $T$-matrix, relating incident and scattered field coefficients 
List of Symbols

\begin{tabular}{|c|c|}
\hline $\mathbf{T}^{11}, \mathbf{T}^{12}, \mathbf{T}^{21}, \mathbf{T}^{22}$ & The blocks of the $T$-matrix \\
\hline$T_{n}$ & $\theta$-derivative of associated Legendre function, with \\
\hline & implicit degree $m$ and argument $\cos \theta$ \\
\hline $\mathbf{U}$ & The $U$-matrix, the irregular part of $\mathbf{Q}$ \\
\hline $\mathbf{U}^{11}, \mathbf{U}^{12}, \mathbf{U}^{21}, \mathbf{U}^{22}$ & The blocks of the $U$-matrix \\
\hline $\mathcal{U}$ & Operator returning odd part of function \\
\hline$V$ & Volume of particle \\
\hline$W_{\mathrm{Abs}}$ & Power absorbed \\
\hline$W_{\text {Ext }}$ & Power extinguished \\
\hline$W_{\text {Sca }}$ & Power scattered \\
\hline$X$ & Arbitrary function \\
\hline$\vec{X}$ & A vector field (real) \\
\hline$\underline{\vec{X}}$ & A vector field (complex) \\
\hline$\underline{X}_{0}$ & Complex field amplitude vector \\
\hline$Z$ & Transformed radial component of VSWF \\
\hline$a$ & Length of semiaxis along $x$ - and $y$ - axes \\
\hline$a$ & Radius of a sphere \\
\hline$a$ & Arbitrary value \\
\hline$\vec{a}$ & Coefficient vector of incident field, magnetic terms \\
\hline$a_{x}$ & $\begin{array}{l}\text { Fraction of surface area responsible for } x \% \text { of the } \\
\text { signal }\end{array}$ \\
\hline$a_{80}$ & $\begin{array}{l}\text { Fraction of surface area responsible for } 80 \% \text { of the } \\
\text { signal }\end{array}$ \\
\hline$a_{\text {in }}$ & Coefficient for Riccati-Bessel function \\
\hline$a_{n m}$ & Coefficients of incident field, magnetic terms \\
\hline$b$ & Arbitrary value \\
\hline$b$ & Quantity relating to geometry of offset sphere \\
\hline$\vec{b}$ & Coefficient vector of incident field, electric terms \\
\hline$b_{i k}$ & Coefficient for Riccati-Bessel function \\
\hline$b_{n m}$ & Coefficients of incident field, electric terms \\
\hline$c$ & Length of semiaxis along $z$-axis \\
\hline$c$ & Vacuum speed of light \\
\hline$c$ & Arbitrary value \\
\hline
\end{tabular}


$\vec{c}$

$c_{i q n k}$

$c_{q}$

$c_{n m}$

$\vec{d}$

$d_{n} \equiv d_{n m} \equiv d_{0 m}^{n}(\theta)$

$d_{n}$

$d_{n m}$

e

e

$e_{p}$

$\tilde{e}_{p}$

$f$

$f$

f

f

$f^{\prime}$

$f^{\prime \prime}$

$f_{1}$

$f_{2}$

$f_{3}$

$f_{\theta}$

$g$

$g$

$g_{\theta}$

$h$

$h$

$h$

$h_{T}$

$h_{n}^{(1)}$
Coefficient vector of scattered field, magnetic terms

Coefficient

Coefficient

Coefficients of scattered field, magnetic terms

Coefficient vector of scattered field, electric terms

Wigner $d$-function

Prefactor

Coefficients of scattered field, electric terms

Eccentricity of spheroid

Arbitrary value

Even part of $x_{\theta} x^{p}$

Even part of $x^{p}$

A function

Generating function for VSWFs

Arbitrary value

Odd part of offset sphere geometry

A function

A function

A function

A function

A function

Derivative with respect to $\theta$ of $f$

Arbitrary value

Even part of offset sphere geometry

Derivative with respect to $\theta$ of $g$

Aspect ratio, ratio of maximum to minimum distances from the origin

Arbitrary value

Arbitrary function

Aspect ratio of spheroid in tip of bipyramid

Spherical Hankel function of the first kind, order

$n$ 
List of Symbols

\begin{tabular}{|c|c|}
\hline & cal Hankel function of the second kind, order \\
\hline & $n$ \\
\hline \multirow{2}{*}{$\begin{array}{l}j \\
j_{n}\end{array}$} & Arbitrary function \\
\hline & Regular spherical Bessel function of order $n$ \\
\hline$k$ & Matrix index \\
\hline$k$ & Wavenumber \\
\hline$\vec{k}$ & Wavevector \\
\hline$\vec{k}_{1}$ & Incident wavevector \\
\hline$k_{1}$ & Wavenumber in surrounding medium \\
\hline$k_{2}$ & Wavenumber in particle \\
\hline$m$ & Matrix index, often implicit \\
\hline \multirow[t]{2}{*}{$m$} & Separation constant, representing projected angu- \\
\hline & lar momentum \\
\hline$m^{\prime}$ & $\begin{array}{l}\text { Matrix index, assumed equal to } m \text { for axisymmet- } \\
\text { ric particles }\end{array}$ \\
\hline$n$ & Matrix index \\
\hline$n$ & Parameter in geometry of modified spheroid \\
\hline$n$ & Refractive index (real part) \\
\hline$n$ & $\begin{array}{l}\text { Separation constant, representing total angular } \\
\text { momentum }\end{array}$ \\
\hline$\hat{n}$ & Unit normal vector \\
\hline$\hat{n}_{A}$ & Unit normal vector to surface $A$ \\
\hline$n_{1}$ & Refractive index in surrounding medium (real) \\
\hline$n_{2}$ & Refractive index in particle (complex) \\
\hline$n_{\phi}$ & Unit normal vector, $\phi$ component \\
\hline$n_{\theta}$ & Unit normal vector, $\theta$ component \\
\hline$n_{r}$ & Unit normal vector, $r$ component \\
\hline$p$ & Integer exponent and index \\
\hline \multirow{2}{*}{$\begin{array}{l}p \\
q\end{array}$} & Parameter in geometry of modified spheroid \\
\hline & Integer exponent and index \\
\hline$q_{\min }$ & $\begin{array}{l}\text { Minimum value } q \text { may take to result in non- } \\
\text { negative exponent }\end{array}$ \\
\hline
\end{tabular}




\begin{tabular}{|c|c|}
\hline$r(\theta)$ & $\begin{array}{l}\text { Angle-dependent distance from origin to particle } \\
\text { surface }\end{array}$ \\
\hline$r$ & Radial distance \\
\hline$\hat{r}$ & Unit vector in the $r$-direction \\
\hline$\vec{r}$ & Position vector \\
\hline$r_{A}$ & $\begin{array}{l}\text { Area-equivalent sphere radius, radius of sphere } \\
\text { having the same surface area as particle }\end{array}$ \\
\hline$r_{\max }$ & Maximum radial distance \\
\hline$r_{\min }$ & Minimum radial distance \\
\hline$r_{p}$ & $\begin{array}{l}\text { Position of intersection of cone and spheroid in } \\
\text { bipyramid }\end{array}$ \\
\hline$r_{V}$ & $\begin{array}{l}\text { Volume-equivalent sphere radius, radius of sphere } \\
\text { having the same volume as particle }\end{array}$ \\
\hline$r_{\theta}$ & Derivative of $r$ with respect to $\theta$ \\
\hline$s$ & Relative refractive index \\
\hline$t$ & Time \\
\hline$u_{p}$ & Odd part of $x_{\theta} x^{p}$ \\
\hline$\tilde{u}_{p}$ & Odd part of $x^{p}$ \\
\hline$u_{r b}$ & Coefficient \\
\hline$v$ & Speed of wave propagation \\
\hline$w$ & Any Riccati-Bessel function \\
\hline$w$ & Any associated Legendre function \\
\hline$w_{n}$ & Any Riccati-Bessel function of explicit order $n$ \\
\hline$w_{r}$ & Coefficient \\
\hline$x$ & Local size parameter \\
\hline$\hat{x}$ & Unit vector of $x$-axis \\
\hline$x_{\max }$ & $\begin{array}{l}\text { The size parameter, maximum value of } x \text { for the } \\
\text { particle }\end{array}$ \\
\hline$x_{\theta}$ & Derivative of $k_{1} r$ with respect to $\theta$. \\
\hline$\hat{y}$ & Unit vector of $y$-axis \\
\hline$y_{n}$ & Irregular spherical Bessel function of order $n$ \\
\hline$z$ & $\begin{array}{l}\text { Position of intersection of cone and spheroid in } \\
\text { bipyramid }\end{array}$ \\
\hline
\end{tabular}


List of Symbols

\begin{tabular}{|c|c|}
\hline$\hat{z}$ & Unit vector of $z$-axis \\
\hline$z_{n}$ & Any spherical Bessel function \\
\hline$\vec{\Gamma}$ & Magnetic susceptibility \\
\hline$\vec{\Delta}$ & Electric susceptibility \\
\hline$\Delta$ & Difference in product of Riccati-Bessels \\
\hline$\Theta(\theta)$ & $\theta$-dependent part of VSWF \\
\hline$\Phi(\phi)$ & $\phi$-dependent part of VSWF \\
\hline$\Omega$ & A subset of a surface \\
\hline$\alpha$ & Coefficient whose value is not important \\
\hline$\alpha$ & $\begin{array}{l}\text { Metric representing the agreement between two } \\
\text { values }\end{array}$ \\
\hline$\alpha$ & Proportionality factor for recursion relation \\
\hline$\alpha$ & Angle used in bipyramid geometry \\
\hline$\alpha_{n m}$ & Coefficients of internal field, magnetic terms \\
\hline$\alpha_{q n k}(x)$ & Term in power series with some prefactor \\
\hline $\bar{\alpha}_{n k}$ & Value of $\alpha_{q n k}$ for $q=q_{\min }$ \\
\hline$\vec{\alpha}$ & Coefficient vector of internal field, magnetic terms \\
\hline$\beta$ & Coefficient whose value is not important \\
\hline$\beta$ & Proportionality factor for recursion relation \\
\hline$\beta$ & Angle used in bipyramid geometry \\
\hline$\beta^{\prime}$ & Coefficient whose value is not important \\
\hline$\beta^{\prime \prime}$ & Coefficient whose value is not important \\
\hline$\beta_{j q}$ & Coefficient \\
\hline$\beta_{n m}$ & Coefficients of internal field, electric terms \\
\hline$\vec{\beta}$ & Coefficients of internal field, electric terms \\
\hline$\gamma$ & Prefactor \\
\hline$\gamma$ & Proportionality factor for recursion relation \\
\hline$\gamma_{q n k}$ & Coefficient \\
\hline$\delta$ & Proportionality factor for recursion relation \\
\hline$\varepsilon$ & Relative permittivity \\
\hline$\varepsilon_{0}$ & Permittivity of free space \\
\hline$\varepsilon_{1}$ & Relative dielectric function of surrounding medium \\
\hline$\varepsilon_{2}$ & Relative dielectric function of particle \\
\hline
\end{tabular}


$\xi_{n}$

$\pi_{n} \equiv \pi_{n m}(\theta)$

$\rho$

$\rho_{f}$

$\underline{\rho}_{f}$

$\left\langle\sigma_{\mathrm{Abs}}\right\rangle$

$\left\langle\sigma_{\text {Ext }}\right\rangle$

$\left\langle\sigma_{\text {Sca }}\right\rangle$

$\sigma_{\mathrm{Abs}}$

$\sigma_{\text {Ext }}$

$\sigma_{\text {Sca }}$

$\tau_{n} \equiv \tau_{n m}(\theta)$

$\phi$

$\phi$

$\phi_{p}$

$\hat{\phi}$

$\chi$

$\chi_{n}$

$\psi_{n}$
Coefficient whose value is not important

Coefficient whose value is not important

Unit vector in the $\theta$-direction

Polar angle

Polar angle of incident field

Coefficient whose value is not important

Refractive index (complex part)

Wavelength

Coefficient whose value is not important

Coefficient whose value is not important

Relative permeability

Permeability of free space

Coefficient whose value is not important

Difference in two values of $F$

Riccati-Hankel of the first kind of order $n$

Angular function

Charge density

Free charge density (real)

Free charge density (complex)

Orientation-averaged absorption cross section

Orientation-averaged extinction cross section

Orientation-averaged scattering cross section

Absorption cross section

Extinction cross section

Scattering cross section

Angular function

Azimuthal angle

Coefficient whose value is not important

Azimuthal angle of incident field

Unit vector in the $\phi$-direction

Electric susceptibility

Irregular Riccati-Bessel function of order $n$

Regular Riccati-Bessel function of order $n$ 
List of Symbols

$\begin{array}{ll}\omega & \text { Angular frequency } \\ \omega_{L} & \text { Angular frequency of incident light } \\ \omega_{R} & \text { Angular frequency of Raman-scattered light }\end{array}$

xxiv 


\section{Introduction}

This thesis presents a collection of results, all having the unifying theme of light scattering by particles. The results may broadly be categorised in two groups, firstly improvements to the extended boundary-condition method (EBCM), and secondly the study of electromagnetic enhancement in the vicinity of metal particles.

Most of the content of this thesis has already been published. It may be found in Refs. [1 7]. Throughout this thesis, all instances of references in this range (7 or less) are to works that form part of this thesis.

\subsection{Motivation and Goals}

The field of computational light scattering finds many uses, but the types of problems that are able to be treated is invariably limited by computational constraints. These may take the form of time constraints, or numerical limitations on the specifics of the problems that may be solved. Irrespective of the method used to consider the light scattering, there is always some limitation. The extended boundary-condition method, which is the subject of much of this thesis, is a fairly general light scattering method that promises to be one of the best choices for solving light scattering problems, especially by axisymmetric particles. This is due to its ability to easily consider all orientations of the problem, and to treat multiple scattering configurations. In addition, the range of shapes it can in principle treat is broad. Of course, it has its own limitations, one of which is a loss of numerical precision, which limits the numerical convergence of results, and in practice, the size and aspect ratio of particles that may be studied. Thus, it was desired to examine these convergence issues, to see where they arise from, and if possible to resolve them. In addition, 


\section{Introduction}

the calculation of certain results, such as local fields, present greater numerical difficulty that other (far-field) quantities, and hence when near-field results are required, attaining convergence can be more challenging.

One particular area where light scattering calculations are of great importance is the field of plasmonics, which concerns light interacting with metal surfaces. The subset of plasmonics of particular interest to this thesis is surface-enhanced Raman spectroscopy (SERS), where the Raman signal is enhanced approximately to the fourth power of the electric field enhancement. This can allow the Raman signal from single molecules to be observed in some cases, which is an active area of research in this research group, which was led by the late Pablo Etchegoin, along with Eric Le Ru. In order to make sense of SERS signals, it is necessary to have an understanding of the surface distribution of the SERS enhancement factors, which is best understood through numerical simulations. One question that is difficult to answer experimentally is how many molecules contribute to a given signal. If the distribution of enhancement factors is better understood, then substrates with more favourable distribution for the specific experiment being carried out can be sought. This would usually relate to how localised the regions of large enhancement are, which leads to one of the primary aims of this work, to understand the factors that effect the localisation of SERS hot-spots.

\subsection{Outline}

Light scattering is the main topic of this thesis, and fittingly Chapter 2 begins by introducing that field, covering both the uses of studying light scattering, as well as various methods that are used. Following that, Chapter 3 includes some detailed background of Mie theory, the EBCM and the $T$-matrix method. The latter of these are the prime focus of theoretical results in this work.

The first chapter of results is Chapter 4, which presents new forms of the integral expressions used in the EBCM for axisymmetric particles. This also contains linear relations between matrix elements from the EBCM, which might allow faster, more efficient calculation of the $T$-matrix. The work in this chapter on the integral expres- 


\subsection{Outline}

sions was published in Ref. [1, and the work on the linear relations was the subject of a talk at the New Zealand Institute of Physics Conference, 2011, in Wellington, but still requires more work to be applied.

The second chapter of results, Chapter 5, focuses almost exclusively on light scattering by spheroids. The EBCM has long been known to suffer from numerical problems, and here we study and identify the cause of these problems for spheroids (and offset spheres), and provide a method to remove the cause of these problems. This work was the focus of Refs. [3, 6], and was presented at the 6th International Conference on Surface Plasmon Photonics, 2013, Ottawa, Canada.

The third and final chapter of results is Chapter 6. This covers the application of light-scattering calculations to plasmonic systems. This is both to support experimental results, as well as to examine the behaviour of field enhancement factors in the vicinity of hot-spots, which are regions of high enhancement. The experimentally-based projects for which some computational support was provided are published in Refs. [2, 5], while the work on hot-spot behaviour has been presented at the 9th Australasian Conference on Vibrational Spectroscopy, 2011, Wellington, the 12th International Conference on Nanotechnology IEEE NANO, 2012, Birmingham, England[4], and the 6th International Conference on Advanced Materials and Nanotechnology, 2013, Auckland.

Chapter 7 presents a brief discussion of the importance of the results, and future work that might stem from this body of work, and concludes the body of this thesis. 



\section{Light Scattering}

』The field of light scattering has a long history, and finds application in many areas of science, along with consequent differences in approach and notation. In this thesis, we focus only on understanding light scattering by particles, for the most part single particles, but with some consideration to scattering by collections of particles. While the term light is used, the results are applicable to all scattering by electromagnetic radiation, not just that which falls in the visible range.

This chapter begins by considering light scattering by particles, and concludes by looking at particular aspects of light scattering of interest in surface-enhanced Raman spectroscopy (SERS). There are many excellent books on these topics, and this chapter provides only an overview of topics relevant to this thesis. For a more detailed and exhaustive explanation of light scattering by particles, the works of Bohren and Huffman[8] and Mishchenko, Travis and Lacis [9] are excellent resources. For the SERS component, aspects of light scattering of interest to SERS (but excluding the mechanics of Raman scattering) are briefly discussed, and for more detail, references such as Le Ru and Etchegoin[10] should be consulted.

\subsection{Applications}

Among the many possible applications of light scattering, a few are presented briefly here. One common use is atmospheric measurements, where scattering of visible to

Pavlova Preheat oven to $150^{\circ} \mathrm{C}$. Beat 4 egg whites in a bowl until soft peaks have formed. Slowly add 1 cup white sugar while beating, until glossy. Sift 3 tsp of cornflour over this, and fold through with 1 tsp vinegar. Place on a baking tray on baking paper, in a heaped pile. Place in oven, and set temperature to $120^{\circ} \mathrm{C}$. Cook for $1 \mathrm{~h}$, then turn off and let cool in oven. Top with whipped cream and seasonal fruit. 


\section{Light Scattering}

infrared light by mainly water droplets and ice crystals in clouds provides information about the size of droplets and location of the clouds[11, 12]. Equally, radio waves reflecting off objects may be used to locate objects, as in radar. While this primarily focuses on analysing the reflection of the incident light, that is not always the case. In astronomical measurements, the observed light must pass through interstellar regions that contain cosmic dust, which scatters the light, and attenuates it [13]. Thus, modelling how light from distant objects is affected by this dust is important for astronomers to obtain correct intensities for these objects. Light-scattering methods are also used to examine how light propagates through the branches and leaves of trees[14]. As well as these far-field effects, it is possible to consider near-field effects, or the combination of the two. The modelling of how a mobile phone operates near a person's head is one such case[15]. Here, both the influence of something in the near-field on the transmitted and received signal is considered, as well as the intensity of the fields in the near field. It is also possible to model light scattering by red blood cells [16]. Another example of near-field interest is in surface-enhanced spectroscopies, where the influence of a metallic surface on the electric field leads to enhanced light scattering by molecules of interest [17, 18]. While the reason for the enhanced scattering is not covered in this thesis, studying the field enhancement is a prime area of focus.

\subsection{General Light Scattering}

The basis for considering light scattering is to solve for the electric and magnetic fields at points in and around the particle of interest, for some incident electric and magnetic fields. Typically, the incident fields correspond to some propagating electromagnetic radiation, satisfying the wave equation.

\subsubsection{Field Expressions}

We begin by making the assumption, appropriate for light scattering problems, that the solution is time-harmonic. That is, the field has a sinusoidal dependence in time 
with angular frequency denoted $\omega$. We may then write some field $\vec{X}(\vec{r}, t)$ as

$$
\vec{X}(\vec{r}, t)=\operatorname{Re}\left(\underline{\vec{X}}(\vec{r}, \omega) e^{-i \omega t}\right)
$$

where $\underline{\vec{X}}(\vec{r}, \omega)$ represents a complex field, and we make use of the $e^{-i \omega t}$ convention for the time dependence. Physical quantities, however, make use of the real field $\vec{X}(\vec{r}, t)$. For non-harmonic fields, these may be composed from the harmonic fields by taking the superposition of harmonic fields, following Fourier analysis. That is, we may consider the complex field to be the Fourier components of the real field, such that the real field may be expressed as

$$
\vec{X}(\vec{r}, t)=\frac{1}{\sqrt{2 \pi}} \int_{-\infty}^{\infty} \underline{\vec{X}}(\vec{r}, \omega) e^{-i \omega t} \mathrm{~d} \omega
$$

with the requirement that $\underline{\vec{X}}(\vec{r},-\omega)=\underline{\vec{X}}(\vec{r}, \omega)^{*}$, where ${ }^{*}$ represents complex conjugation. Similarly, the complex components may be obtained from the real field as

$$
\underline{\vec{X}}(\vec{r}, \omega)=\frac{1}{\sqrt{2 \pi}} \int_{-\infty}^{\infty} \vec{X}(\vec{r}, t) e^{+i \omega t} \mathrm{~d} t .
$$

The result of the above is that in this thesis fields are treated as monochromatic, harmonic ones, but other forms of field may be constructed from them, with results following from the linearity of Maxwell's equations, and the assumed linear response of the materials we are using.

\subsubsection{Maxwell's Equations}

The solutions to the light-scattering problem must obey Maxwell's Equations, which may be written (in SI units) as

$$
\begin{aligned}
\nabla \cdot \vec{E} & =\frac{\rho}{\varepsilon_{0}} \\
\nabla \times \vec{E}+\frac{\partial \vec{B}}{\partial t} & =0 \\
\nabla \cdot \vec{B} & =0
\end{aligned}
$$




\section{Light Scattering}

$$
\nabla \times \vec{B}=\mu_{0}\left(\vec{J}+\varepsilon_{0} \frac{\partial \vec{E}}{\partial t}\right)
$$

These contain several terms, namely the electric field $\vec{E}$, the magnetic induction $\vec{B}$, the permittivity of free space $\varepsilon_{0}$, and the permeability of free space $\mu_{0} . \quad \rho$ is the charge density, and $\vec{J}$ is the current density. These equations are valid on a microscopic scale (the so-called "microscopic Maxwell's equations"). It is, however, challenging to apply these to complicated matter made of atoms, with local chargeand current-density. In this case, it is preferable to instead make use of the "macroscopic Maxwell's equations",

$$
\begin{aligned}
\nabla \cdot \vec{D} & =\rho_{f} \\
\nabla \times \vec{E}+\frac{\partial \vec{B}}{\partial t} & =0 \\
\nabla \cdot \vec{B} & =0 \\
\nabla \times \vec{H} & =\vec{J}_{f}+\frac{\partial \vec{D}}{\partial t} .
\end{aligned}
$$

Here the fields $\vec{D}$ and $\vec{H}$ are the electric displacement and magnetic field respectively. These take into account the contribution of the internal charges, that is, the response of the material to the incident field. These are related to the electric field $\vec{E}$ and the magnetic induction $\vec{B}$ by

$$
\begin{aligned}
& \vec{D}=\varepsilon_{0} \vec{E}+\vec{P} \\
& \vec{H}=\frac{\vec{B}}{\mu_{0}}-\vec{M}
\end{aligned}
$$

where $\vec{P}$ is the electric polarisation and $\vec{M}$ is the magnetisation. The quantities $\rho_{f}$ and $\vec{J}_{f}$ are the charge density and current density that are associated with external charges (that is, the ones that generate the incident field, and so are zero in the regions that we consider), while $\rho$ and $\vec{J}$ (as in Eqs. 2.4 and 2.7) respectively) include the internal charges also.

It is possible to write these Maxwell's equations in terms of the Fourier compo- 
nents of the field, as

$$
\begin{aligned}
\nabla \cdot \underline{\vec{D}} & =\underline{\rho}_{f} \\
\nabla \times \underline{\vec{E}}-i \omega \mu_{0} \underline{\vec{H}} & =0 \\
\nabla \cdot \underline{\vec{H}} & =0 \\
\nabla \times \underline{\vec{H}} & =\underline{\vec{J}}_{f}-i \omega \underline{\vec{D}},
\end{aligned}
$$

where here we work with the frequency-dependent complex fields.

In order to make use of the macroscopic Maxwell's equations, we must be able to relate the response of the material $\vec{P}, \vec{M}$ with the excitation $\vec{E}$ and $\vec{H}$. For this we use the so-called constitutive relations,

$$
\begin{aligned}
& \underline{\vec{B}}(\omega)=\mu_{0} \mu(\omega) \underline{\vec{H}}(\omega) \\
& \underline{\vec{P}}(\omega)=\varepsilon_{0} \chi(\omega) \underline{\vec{E}}(\omega)
\end{aligned}
$$

with the permeability $\mu$ and the electric susceptibility $\chi$ being material properties of the medium. Thus the fine details of the response of the material, the polarisation and the magnetisation, are included in the susceptibility and permeability. The regime being considered here assumes that the response (that is, $\underline{\vec{B}}, \underline{\vec{P}}$ ) is linear with respect to the field, and so $\mu$ and $\chi$ are independent of the field. This assumption does rule out some materials from consideration (such as those with large secondorder responses), but it covers the vast majority of materials, including those of interest to this thesis (gold and silver primarily). We also assume that the response is local in space (independent of the field at other points), but non-local in time (with proper consideration to the time-harmonic nature of the fields). We note that by using the expression for the polarisability, we may write the expression for the electric displacement as

$$
\underline{\vec{D}}=\varepsilon_{0}(\underline{\vec{E}}+\chi \underline{\vec{E}})=\varepsilon_{0} \varepsilon(\omega) \underline{\vec{E}}
$$

where we define the relative dielectric function (often referred to simply as the 


\section{Light Scattering}

dielectric function)

$$
\varepsilon(\omega)=1+\chi(\omega)
$$

It is worth noting that the dielectric function is in general a function of frequency, and can have a strong frequency dependence. We make the assumption that the materials we use are non-magnetic, that is, both $\mu=1$ and $\vec{M}=\overrightarrow{0}$, and so the permeability is not frequency dependent. We also assume that the particles are embedded in a homogeneous, isotropic, non-absorbing medium.

The charge and current terms in Eqs. 2.14 -2.17) are the external ones, and so are zero in the region of interest. These equations then reduce to the Helmholtz equation [8] (or vector wave equation) for the divergence-less electric field,

$$
\left\{\begin{array}{l}
\nabla^{2} \vec{E}(\vec{r}, \omega)+k^{2} \vec{E}(\vec{r}, \omega)=\overrightarrow{0} \\
\nabla \cdot \vec{E}=0
\end{array}\right.
$$

which is valid only on the complex fields as discussed earlier. Here, the value $k$ is the wave vector, and is given by

$$
k^{2}=\frac{\omega^{2}}{c^{2}} \varepsilon(\omega)
$$

where $c$ is the speed of light in vacuum. In the surrounding (and non-absorbing medium), the speed of the wave propagation is given by

$$
v=\frac{\omega}{k \sqrt{\varepsilon(\omega)}}
$$

where $\sqrt{\varepsilon(\omega)}=n(\omega)$, the refractive index. For materials with absorption (which might include the material of the particle, but excludes the surrounding medium), then instead we have the complex refractive index

$$
\sqrt{\varepsilon(\omega)}=n(\omega)+i \kappa(\omega)
$$

where $n(\omega)$ represents the speed of propagation through the medium, and $\kappa(\omega)$ 
represents the absorption of light in the medium. We restrict ourselves to nonactive media, so that $\kappa(\omega) \geq 0$.

\subsubsection{Plane Waves}

In the case of incident fields, we work with plane waves. Other incident waveforms may be constructed by considering superpositions of plane waves, and again making use of the linearity of Maxwell's equations. Plane waves are waves which vary along some incident wavevector $\vec{k}$, but do not vary perpendicular to $\vec{k}$. We have, for the incident waves, only real $\vec{k}$. With the requirement that these waves are harmonic in both space and time, we may write an equation for these waves as

$$
\underline{\vec{X}}(\vec{r})=\underline{\vec{X}}_{0} e^{i \vec{k} \cdot \vec{r}}
$$

(the time-dependence is introduced when obtaining the real field). Here $\underline{X}_{0}$ is a complex constant vector. If we assume that the electric field is defined as

$$
\underline{\vec{E}}=\underline{\vec{E}}_{0} e^{i \vec{k} \cdot \vec{r}}
$$

then by using Eq. 2.15 we can obtain $\underline{\vec{H}}$ as

$$
\underline{\vec{H}}=\underline{\vec{H}}_{0} e^{i \vec{k} \cdot \vec{r}}
$$

Note that here, $\underline{\vec{H}}_{0}$ is not independent of $\underline{\vec{E}}_{0}$.

Now that we have some incident wave, we need to consider the situation of interest. That is, these incident waves must be incident on some scatterer. Schematically, this may be seen in Fig. 2.1. Here we have a plane wave incident on a single, arbitrary scatterer. We shall assume for simplicity that the scatterer is homogeneous, and the response of it (that is, $\varepsilon(\omega)$ ) is isotropic, which is an assumption used in all of this thesis.

The polarisation of the incident wave is described by the constant vectors $\underline{E}_{0}$ and $\underline{\vec{H}}_{0}$. In general, the response of the system will depend on the polarisation of the incident radiation. The problem that we wish to solve is to determine the electric 


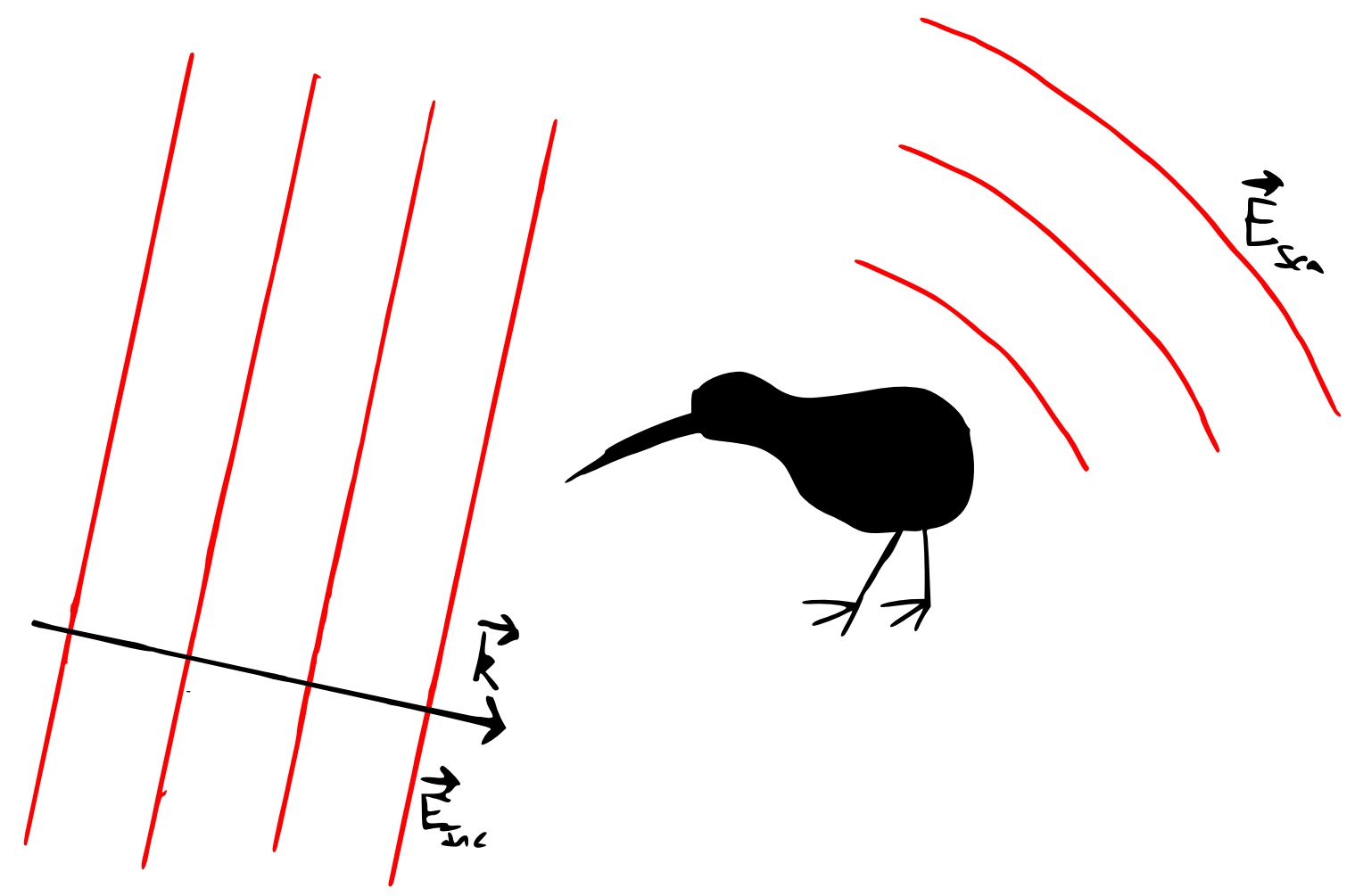

Figure 2.1: A schematic showing a plane wave incident on a scatterer. Here the incident plane wave (in red) with wavevector $\vec{k}$ is incident on the scatterer (here a kiwi). The field is scattered, with some of the scattered field depicted as outgoing spherical waves.

field at various points, given some incident field. Using Eq. 2.22), it is sufficient to solve for only the electric field, and then Eq. 2.15) may be used to obtain the magnetic field as required. Different techniques that are commonly used to solve light scattering problems are discussed in Section 2.4.

\subsubsection{Boundary Conditions}

As with many problems in physics, it is the boundary conditions of the problem that make it interesting and challenging to solve. In this case, the boundary conditions are supplied by Maxwell's equations. We have in general a discontinuity in the value of $\varepsilon$ at the boundary (and for magnetic media, not considered here, there is also a 
discontinuity in $\mu$ ). The requirements are that

$$
\begin{aligned}
& \left(\vec{E}_{1}-\vec{E}_{2}\right) \times \hat{n}=0 \\
& \left(\vec{H}_{1}-\vec{H}_{2}\right) \times \hat{n}=0
\end{aligned}
$$

at points on the surface of the particle, where $\hat{n}$ is the (outward) unit normal to the surface. These statements are that the components of the electric and magnetic fields that are perpendicular to the normal (or parallel to the surface) are continuous. As we are more interested in dealing only with the electric components, we may instead used a different expression,

$$
\left(\vec{D}_{1}-\vec{D}_{2}\right) \cdot \hat{n}=0
$$

which is the requirement that the normal component of the electric displacement must be continuous across the boundary. As we have a relation between $\vec{E}$ and $\vec{D}$, we may express this requirement in terms of $\vec{E}$, which is not shown here.

It is common to solve for the scattered field $\vec{E}_{\text {Sca }}$ (in the region outside the particle). From this, the total external field is obtained as the sum of the scattered and incident fields, $\vec{E}_{\text {Ext }}=\vec{E}_{\text {Sca }}+\vec{E}_{\text {Inc }}$. We require that the scattered field must obey the Sommerfeld radiation condition, that the fields must decay as $e^{i k r} / r$ as $r$ goes to $\infty$, meaning that the field at large distances from the particle should look like outgoing spherical waves[19]. Causality excludes the possibility of incoming waves at infinity in the scattered field.

\subsubsection{Scattering Quantities}

While the light scattering problem involves in principle solving for the fields, there are other quantities of interest that are often calculated.

Many of the terms of interest, and arguments used to derive them, are concerned with energy, and energy conservation. The starting point for looking at energy flow 


\section{Light Scattering}

is the time-averaged Poynting vector, which is

$$
\vec{S}=\operatorname{Re}\left(\underline{\vec{E}} \times \underline{\vec{H}}^{*}\right) / 2
$$

and in the case of a plane wave this reduces to

$$
\vec{S}=\frac{\vec{k}}{2 \omega}|\underline{\vec{E}}|^{2}
$$

We may use this Poynting vector to consider the flow of energy in the system. If we consider the quantity

$$
W_{\mathrm{Abs}}=-\int_{A} \vec{S} \cdot \hat{n}_{A} \mathrm{~d} A
$$

on some surface $A$ that fully encloses our particle, and where $\hat{n}_{A}$ is the unit normal on the surface $A, W_{\mathrm{Abs}}$ represents the net energy flow rate into the area enclosed by $A$. As the medium that we are using is non-absorbing, that means that $W_{\mathrm{Abs}}$ is equal to the rate of energy absorption by the particle.

It is also possible to consider the rate of energy scattering by the particle. This is energy that is "lost" from the incident beam, but not absorbed by the particle. Instead, it is scattered by the particle. We may write this

$$
W_{\text {Sca }}=\int_{A} \vec{S}_{\text {Sca }} \cdot \hat{n}_{A} \mathrm{~d} A
$$

where $\vec{S}_{\text {Sca }}$ is the appropriate Poynting vector for the scattered field.

The combination of power that is absorbed and energy that is scattered is the energy that is extinguished from the beam, called the extinction. Thus,

$$
W_{\mathrm{Ext}}=W_{\mathrm{Abs}}+W_{\mathrm{Sca}} .
$$

All of these quantities are often referred to by their cross sections, that is, the equivalent area of the incident beam that provides the same amount of power as is absorbed, scattered or extinguished. These are given the symbols $\sigma_{\mathrm{Abs}}, \sigma_{\mathrm{Sca}}$ and $\sigma_{\mathrm{Ext}}$ 
respectively. The relation between the power absorbed, scattered or extinguished and the cross sections is, for the example of scattering

$$
W_{\text {Sca }}=\sigma_{\text {Sca }} S_{\text {Inc }}
$$

where $S_{\text {Inc }}$ is the incident power density, which in the case of a plane wave is

$$
S_{\text {Inc }}=\frac{1}{2} \varepsilon_{0} c n\left|\vec{E}_{\mathrm{Inc}}\right|^{2} \text {. }
$$

Here $c$ is the speed of light in vacuum, and $n$ is the refractive index of medium.

Other commonly-used metrics are the absorption, scattering or extinction efficiencies, denoted $Q_{\mathrm{Abs}}, Q_{\mathrm{Sca}}$ and $Q_{\text {Ext }}$ respectively. These are simply the cross sections normalised by the geometric cross section of the particle perpendicular to the incident plane wave. These then measure what fraction of the area of the particle scatters, absorbs, or extinguishes, the total (incident + scattered) wave. This may be more that one, perhaps non-intuitively, as it is possible for the particle to have an effect that extends outside its geometric extent.

Following on from the cross sections above, it is worth mentioning another set of quantities, the orientation-averaged cross sections. While the cross sections above depend in general on the orientation of the particle relative to the incident wave, the orientation-averaged values take into account all incident orientations. This might represent measurements taken on a single particle that is rotating on a timescale much faster than the measurements, or a collection of particles with random orientations, so long as the particles are sufficiently well separated that the scattering processes may be considered independent.

In some cases, rather than an orientation-averaged cross section, it is desirable to have the cross section for some specific angle. In the context of radar, the backscatter cross section is important, including for meteorological studies[20]. One matrix that is of interest is the scattering matrix [9], which is able to characterise the complete far-field scattering problem for a given scatterer, but is not a quantity used in near-field studies. 


\section{Light Scattering}

\subsubsection{Far-Field Measurements}

In this section we briefly cover typical scattering measurements that may be performed. The most common one is the extinction measurement, where light is shone through a sample, and the intensity of the transmitted light is measured, as depicted in Fig. 2.2. Note that the light that is collected on the other side of the sample will include some component of scattered light as well as the unscattered light, but the quantity that falls upon a sufficiently small detector is negligible. This measures the combination of scattering and absorption by the sample, though it is in some contexts referred to (incorrectly) as an absorption measurement, as in the case of small molecules the scattering is negligible.

It is also possible to measure the scattering intensity from a sample. This may be achieved by placing a detector at some angle from the incident beam (except in the exact forward direction, where it is not possible to distinguish the scattered and unscattered light). In this way, it is common to map out the angular dependence of the scattering intensity. A schematic of this method is given in Fig. 2.3 .

Another possible means of observing scattering is dark-field microscopy, which is used to visualise scatterers. In this technique, depicted in Fig. 2.4, light is incident on some scatterer from a large angle, and the collected light excludes the incident light, so that only scattered light is observed. This allows us to differentiate between strong scatterers and a typical substrate with a low scattering cross section. It is worth noting that in most experiments, it would be expected that any particles being measured in solution would be rotating, and so results would correspond to orientation-averaged ones. However, if the scatterer is deposited on a substrate then its orientation may be fixed.

Aside from scattering and extinction, it is also possible to measure absorption. This may be done by collecting all of the scattered and transmitted light, and simply taking the difference. An additional tool is to use an integrating sphere, where, the sample is enclosed in a high-reflectivity cavity, and light is shone in one port, and collected out another. The principle is that the light passes through the sample many times, which provides a spectral measurement of the absorption of the sample. Another option is to measure the temperature change of a sample when 


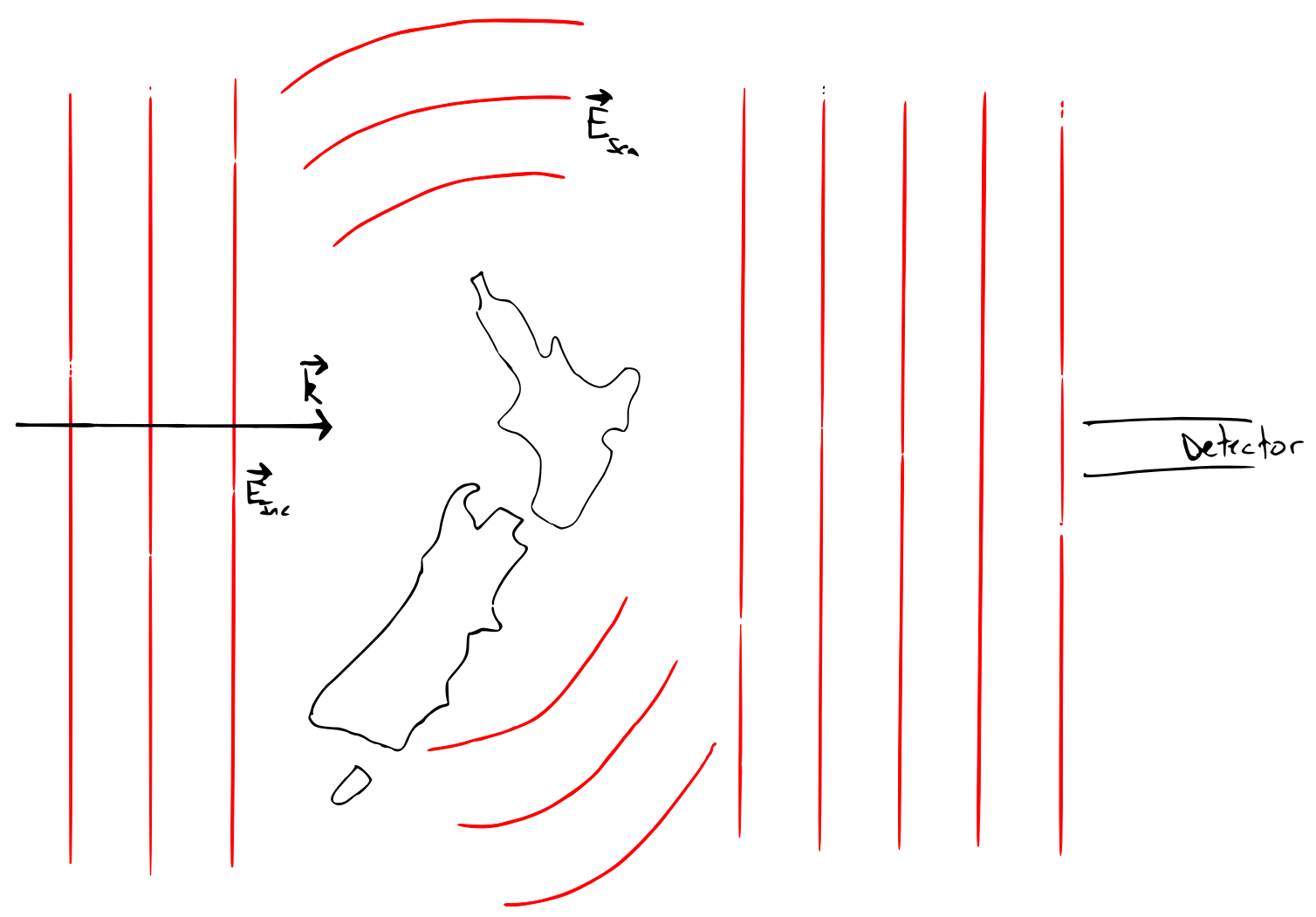

Figure 2.2: Schematic of a typical of extinction measurement. Here some plane wave (depicted as incident from the left) interacts with a collection of scatterers (from top to bottom, the North Island, the South Island, and Stewart Island), leading to some scattering (depicted to the North and South), as well as transmission of the wave. The detector measures the transmitted intensity of this wave, and hence deduces the amount extinguished from the beam. 


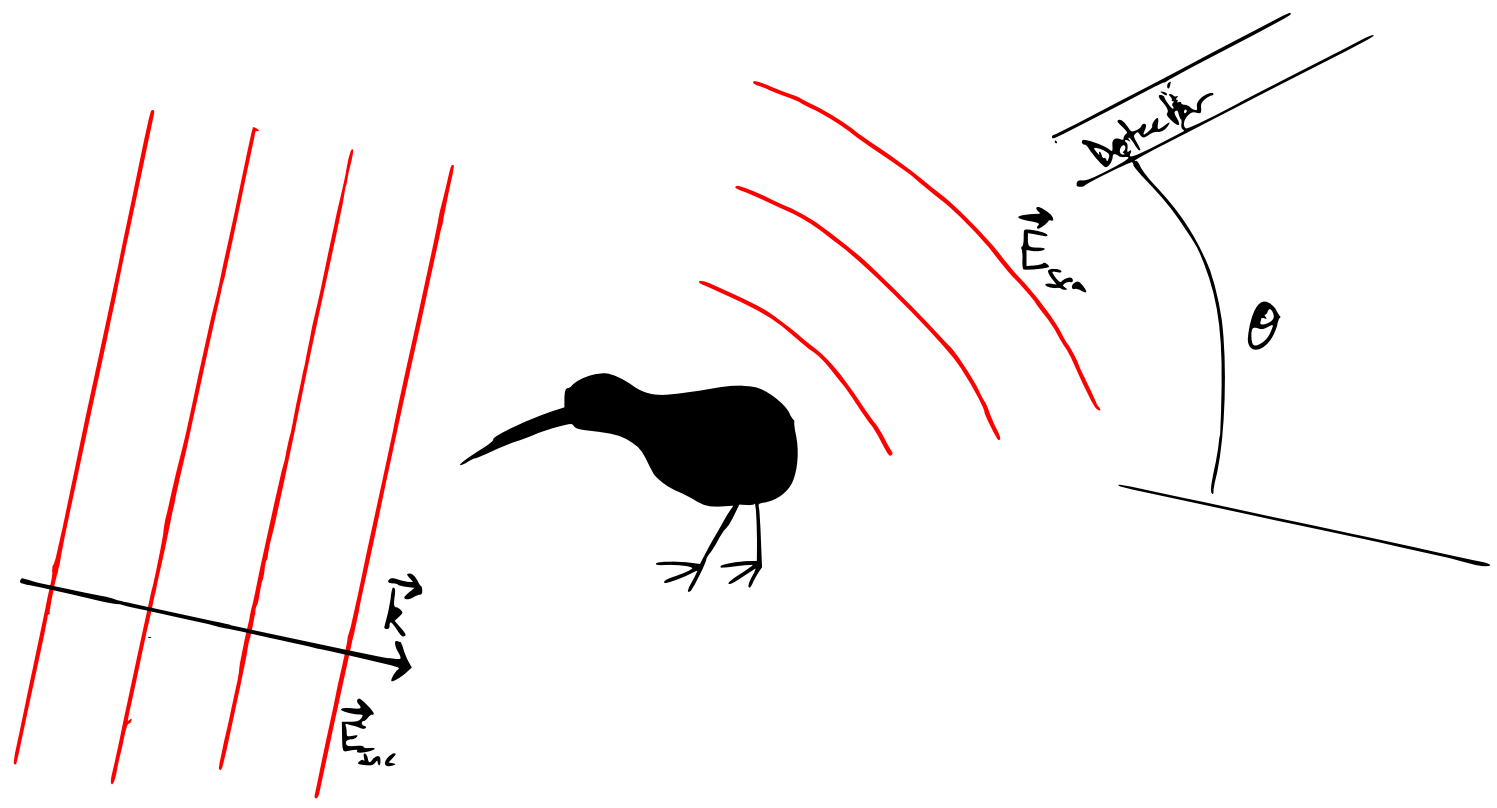

Figure 2.3: Schematic of an angular-dependent scattering measurement. Here the same scatterer as in Fig. 2.1 was used, except now the intensity of the light at some angle $\theta$ with respect to the incident field direction is measured. The detector is collimated, such that the incident field is not collected, and only the scattered field is measured. The prevents measurements near $\theta=0$ from being taken, as here the incident and scattered waves are colinear.

light is shone onto it, using calorimetry. Energy that is absorbed will increase the temperature of the sample. However, with this method, it is not possible to get any frequency resolution, so monochromatic light sources must be used to obtain that information.

\subsection{Surface-Enhanced Raman Spectroscopy}

We now turn to consider the light scattering problem from the perspective of surfaceenhanced Raman spectroscopy (SERS). This couples both far-field and near-field considerations. We begin by looking briefly at Raman scattering, and then examine aspects of scattering by particles that are relevant for SERS. 


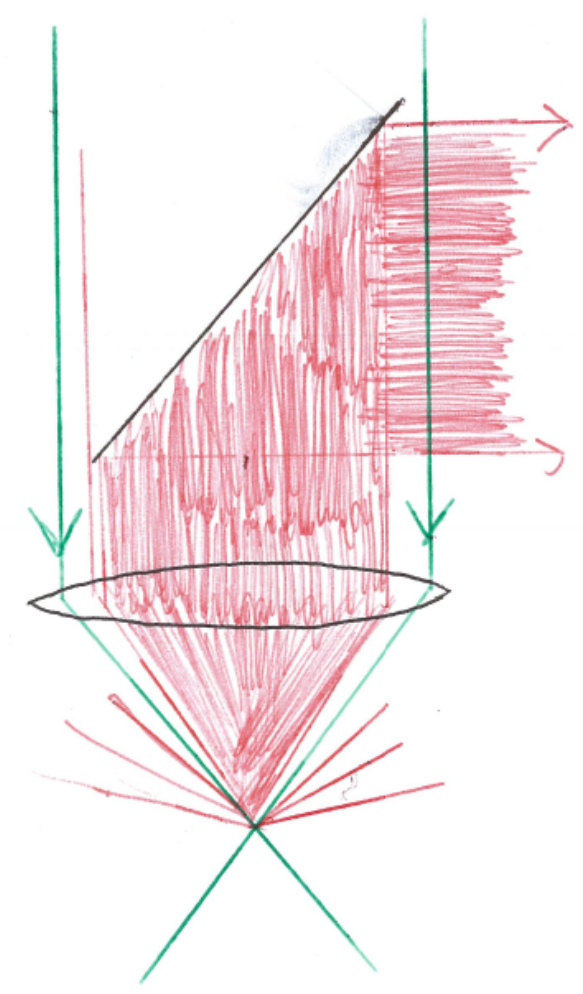

Figure 2.4: Schematic of the dark-field scattering experiment. Here the incident (green) rays incident on the sample fall outside the angle that is directed into the detector. The scattered light (here shown in red) is collected by the lens, and directed into the detector. This allows for the detection of scattering by the object while removing the signal of reflection from the sample or substrate.

\subsubsection{Raman Scattering}

Raman scattering is a variety of inelastic scattering of light, either by molecules or by crystals. In the context of SERS, it is almost exclusively used in the context of molecular scattering, whereby incident light excites vibrations in the molecules and is scattered with a lesser energy, in so-called Stokes scattering. There is also a process, anti-Stokes scattering, where an existing vibration in the molecule gives up its energy to a photon, and the photon energy increases. The energies of the Stokes and anti-Stokes lines are symmetric about the incident energy, but the intensities differ owing to the need to have vibrational states populated to observe anti-Stokes 


\section{Light Scattering}

lines.

These energy differences correspond to various vibrational modes of the molecule, and the resulting spectrum provides a fingerprint of the molecule, which may be used for identification, or to study the bonds in the molecule which are responsible for the vibrations.

Raman scattering is typically a weak process, and competes with fluorescence, which is often a much stronger process, especially in the case of resonant molecules. Here, the energy of the Raman vibration corresponds to an electronic transition of the molecule, leading to both a stronger Raman intensity and a stronger fluorescence intensity. The fluorescence might arise from the molecule that is being studied itself, or from impurities in the sample, which in either case can overwhelm the Raman signal. Typical cross sections are of the order of $10^{-15} \mathrm{~cm}^{2} \mathrm{sr}^{-1}$ for fluorescence, $10^{-30} \mathrm{~cm}^{2} \mathrm{sr}^{-1}$ for non-resonant Raman scattering, and $10^{-24} \mathrm{~cm}^{2} \mathrm{sr}^{-1}$ for resonant Raman. A proper description of the mechanisms and intricacies of Raman spectroscopy is well outside the scope of this work, and interested readers are directed to more specialised works, such as that of Long[21].

\subsubsection{Surface-Enhanced Raman Spectroscopy}

The relative weakness (in terms of intensity) of Raman scattering is one motivation for the use of surface-enhanced Raman scattering. This technique, discovered in 1974[22], makes use of the enhancement of the electromagnetic field that may be observed near a metallic surface. This has two effects. Firstly, and of most interest, is that the Raman scattering cross section of the molecules is altered, and can increase by many orders of magnitude, up to $10^{11}[23$. This allows, for example, the spectrum from a single molecule to be observed 24 26]. The other area of interest is in studying the interaction of molecules with the surface. As SERS requires the molecules to be near the surface for large enhancements, the signal comes primarily from molecules adsorbed to the surface, and as the Raman effect examines bonds, the bonds with the surface influence the spectra obtained[27, 28].

The mechanism of SERS enhancement is a matter of some contention [29, 30]. 
Primarily, however, the effect is electromagnetic, and this will be assumed to be responsible for the entire enhancement in this thesis. The electromagnetic enhancement was suggested by Moskovits[31], and results from couplings between the incident field and the substrate, and the excited Raman field and the substrate. Briefly, the incident field $\vec{E}_{\text {Inc }}$ is modified by the surface to produce some local field $\vec{E}_{\text {Loc }}$. This local field excites a Raman dipole, which would in the total absence of the surface emit with a power proportional to $\left|\vec{E}_{\mathrm{Loc}}\right|^{2}$. From the point of view of the excitation, this power in the presence of the surface increases by a factor of

$$
M_{\mathrm{Loc}}=\frac{\left|\vec{E}_{\mathrm{Loc}}\right|^{2}}{\left|\vec{E}_{\mathrm{Inc}}\right|^{2}}
$$

the local field intensity enhancement factor. However, that is not the entire story. The emission is also affected, by reciprocity arguments by the same factor, with one difference. As the emission is at a different frequency, $M_{\text {Loc }}$ is different. Thus, with frequency-dependence introduced, the enhancement may be written

$$
F\left(\omega_{R}, \omega_{L}\right)=M_{\mathrm{Loc}}\left(\omega_{R}\right) M_{\mathrm{Loc}}\left(\omega_{L}\right)=\frac{\left|\vec{E}_{\mathrm{Loc}}\left(\omega_{R}\right)\right|^{2}\left|\vec{E}_{\mathrm{Loc}}\left(\omega_{L}\right)\right|^{2}}{\left|\vec{E}_{\mathrm{Inc}}\right|^{4}}
$$

where $\omega_{L}$ is the frequency of the incident light, and $\omega_{R}$ is the frequency of the Raman mode being considered. For simplicity, it is often assumed that the Raman enhancement is simply

$$
F(\omega)=M_{\mathrm{Loc}}(\omega)^{2}=\frac{\left|\vec{E}_{\mathrm{Loc}}\right|^{4}}{\left|\vec{E}_{\mathrm{Inc}}\right|^{4}}
$$

which is approximately true for Raman modes close in frequency to the incident light. In fact, all of these equations are at best an approximation, and a more detailed discussion of factors that influence the enhancement factor can be found in Ref. [32].

The result of the above is that the Raman signal is roughly proportional to the 


\section{Light Scattering}

fourth power of the electric field enhancement, which can lead to significant gains in sensitivity. In order to calculate expected enhancement factors, it is necessary to calculate electric fields near the surface of our scatterers. In this instance, 'near' is within a few nanometres of the surface, as SERS signals originate primarily from molecules adsorbed to the surface. As a result, the area of interest in SERS calculations is much more focused near the particle than other light-scattering calculations (as discussed in previous sections), which are principally concerned with far-field measurements.

\subsection{Computational Techniques}

In this section, a brief overview of a variety of computational techniques used to solve the light scattering problem is presented. For a more complete and in-depth examination of this topic, other works are recommended[33]. In this section, only a brief overview is given for the most part, with more detail provided for the methods that were used for this thesis, namely Mie theory, the $T$-matrix method, and finiteelement modelling.

\begin{tabular}{c|c} 
Method & Category \\
\hline Mie theory & Analytical \\
T-matrix method/EBCM & Semi-analytical \\
Surface-integral equation method & Specialised numerical \\
Discrete-dipole approximation & Specialised numerical \\
Finite-element method & General numerical \\
Finite-difference time-domain & General numerical
\end{tabular}

Table 2.1: Classes of different methods for solving light scattering problems

Broadly, the various different techniques can be classed into four groups, though the boundaries between these may be a bit blurry. A categorisation of some techniques is given in Table 2.1. The first two categories, analytical and semi-analytical, do not require discretising the particles under study. They are in principle fast techniques, that are only used for solving electromagnetic problems. The reason that the $T$-matrix is categorised as semi-analytical here is that while the theory is 
analytical, the need to numerically evaluate integrals and invert matrices removes the ability to treat it fully analytically. Both Mie theory and the $T$-matrix method require surfaces that are defined analytically, which restricts the shapes that they are able to model.

Among the numerical methods, there are two categories. The specialised numerical methods are specifically for solving electromagnetic scattering problems, and involve discretising the surface (such as the surface integral equation method) or volume (discrete-dipole approximation) into small elements. This allows the shapes considered to be general. They then make use of known properties of the lightscattering problem to obtain a solution (by making use of Green's functions), and perform this faster than the general numerical solvers. They also, by making use of some properties of the problem, are able to avoid discretising the medium surrounding the particle, and also to ensure that the radiation condition at infinity is satisfied.

The last category of solver is the general numerical solver, which is not specialised to solving electromagnetic problems, but instead is a differential equation solver, capable of treating many different problems. These discretise both the particle and a region of the surrounding medium, which must be large enough so that the field at the edges of the simulation volume is behaving as in the far-field, so that the finite size of the volume does not affect the results. This allows for arbitrary shapes to be simulated, though owing to the lack of specialisation to the light-scattering problem, the time and memory requirements for this method can be large.

\subsubsection{Mie Theory}

In this section only the basic principles of Mie theory are explained. More detail is found in Chapter 3. Mie theory (also called Lorenz-Mie theory), was published in 1908 by Mie 34. This solves the light scattering problem in the case of a spherical scatterer.

The starting point of Mie theory is to express the electric fields in terms of a basis of vector spherical wavefunctions. The scattered and incident fields are expanded 


\section{Light Scattering}

in terms of irregular wavefunctions (that satisfy the Sommerfeld radiation condition that the field goes as $e^{i k r} / r$ as $r$ goes to $\infty$ ), while the internal field is expressed in terms of regular wavefunctions (that are finite at the origin). The coefficients of these series, obtained by applying the boundary conditions, are then related to each other by the Mie coefficients. These Mie coefficients are simply composed of spherical Bessel functions, and are not computationally demanding. The various cross sections are computed as appropriate sums over the Mie coefficients.

Mie theory is able to (with some modification) treat collections of spheres, as well as layered spheres.

\subsubsection{T-matrix Method}

The $T$-matrix method is similar to Mie theory, in that the fields are expanded in terms of a basis of vector spherical wavefunctions. However, this theory allows for non-spherical scatterers, and reduces to Mie theory for spherical scatterers. The eponymous $T$-matrix relates the coefficients of the expansion of the incident and scattered fields, and there is another matrix (which we will call $\mathbf{R}$ ) which relates the incident and internal fields. One of the benefits of this method is that, as the $T$-matrix relates the coefficients of the fields, any incident field may be used, and so different field orientations are easy to model once the matrix has been calculated. The same is true for Mie theory, but owing to the spherical symmetry in that case it is not significant. A more thorough description of the $T$-matrix method is provided in Chapter 3 .

The $T$-matrix is usually calculated using the extended boundary-condition method (EBCM) (also known as the null-field method), which involves integrals on the surface of the scatterer. In the case of particles with symmetry of revolution, these surface integrals reduce to line integrals, which are much simpler. There is also a matrix inversion step in order to obtain the $T$ matrix.

It is possible to obtain the $T$-matrix through other methods, such as the discretedipole approximation [35] or point-matching method[36], though the EBCM is the most common approach. There are also implementations of the EBCM which take 
finite symmetries into account, allowing more efficient calculation in that case [37].

As with Mie theory, the $T$-matrix method is also able to model scattering by multiple scatterers, as well as layered particles. The $T$-matrix method is able to calculate scattering properties for any incident direction, and it is also possible to perform analytical orientation averaging of far-field scattering properties.

\subsubsection{Finite-Element Method}

The finite-element method (FEM) is a method for solving Maxwell's equations (or other differential equations) for "arbitrary" geometries. In this section, the focus is on the commercial software package COMSOL[38], which makes use of the finiteelement method. COMSOL is able to solve arbitrary differential equations, and has several sets, including Maxwell's equations in media, pre-configured.

The basic principle of operation is to define the simulation geometry, and then to divide it up into a mesh of many smaller elements (which may be irregular). The simulation (for only one incident orientation) then solves Maxwell's equations on each mesh element. The solution on each element uses a quadratic approximation, so the mesh needs to be fine enough that this approximation is good. The simulation geometry here refers to the particle and surrounding medium, which must be large enough that the incident field is not appreciably affected by the edge of the simulation volume.

It is possible to carry out a range of different simulations, and of interest in this work are 3D, full-wave problems, 3D electrostatic problems, and 2D problems, including $2 \mathrm{D}$ axisymmetric ones (which are a $3 \mathrm{D}$ problem where there is no $\phi$ dependence), though other situations are possible. The memory and time requirements to simulate a 3D case are substantially higher than for $2 \mathrm{D}$, and so in some cases the $2 \mathrm{D}$ simulation is preferred. In that case, the electrostatics approximation must be used (the $k$-vector of the full-wave simulation breaks the rotational symmetry, precluding the use of full-wave solutions), and the range of incident orientations is restricted to only the two directions along the axis of revolution.

COMSOL is able to simulate arbitrary geometries, and hence can simulate mul- 


\section{Light Scattering}

tiple particles as well as layered particles. However, with small gaps or regions of high curvature, the meshing requirements become higher and so memory and time requirements increase. Sufficiently challenging geometries are then not able to be solved by some given computer, and a more capable machine may be required. However, with these limitations excepted, the finite element method presents the most general, brute-force method of solving Maxwell's equations for arbitrary geometries.

An alternative to the frequency-domain calculations is to solve in the time domain. This entails modelling an incident pulse, which contains all frequencies, and solving in steps of time[39]. This also requires modelling the particle and the region surrounding, and the dielectric function of both must be known for a wide wavelength range, which in practice can be limiting.

One of the benefits of the finite-element method as compared to other numerical techniques is that the mesh elements do not need to be the same size, so that in regions with rapidly-changing fields, the mesh can be made finer, so the results in that region better approximate the real field. This allows for regions of high curvature to be modelled without imposing the required finer mesh on the entire geometry, with the associated computational cost. This is particularly important when considering the near-field.

\subsubsection{Other Methods}

There are a plethora of other methods that have been developed to solve the lightscattering problem. While they have not been used in the works involved in this thesis, nonetheless they are widely employed in a variety of fields, and a selection of them are briefly presented here.

\section{Discrete Dipole Approximation}

The discrete dipole approximation (DDA) has long been used in light-scattering calculations [40, 41], initially for use in dielectrics but also now applied to metallic particles [42]. This method begins by discretising the particle into small elements, 
each of which is represented as a dipole with some polarisability. The interaction of all of the dipoles with the incident light and each other is then solved to calculate the electric field. This method works well for arbitrarily shaped particles, and can calculate scattering by collections of particles. However, the calculations must be recalculated for different incident orientations.

The accuracy of the results using the DDA is typically good for far-field properties, where a collection of dipoles closely approximates the response of the scatterer. However, for the near-field, the field is not well modelled by a collection of dipoles, and the accuracy becomes reduced, and so the number of dipoles being considered must be increased, substantially in some cases. It is, however, possible to model the near-field using the DDA[43].

\section{Surface Integral Equation Method}

While the discrete dipole approximation is based on discretising the volume, the surface integral equation method (SIE) discretises the surface of the particle into triangles [44]. This allows particles of arbitrary shape to be modelled. The method solves for surface electric and magnetic currents that produce the same field as are produced by the particle volume. The field in the region around (and inside) the particle may then be calculated. SIE has historically found use in the microwave scattering community, but has more recently found favour in the light-scattering community as well 44 46].

This technique, while a recent addition to the light-scattering arsenal, promises to be an efficient technique for computing near-field properties of arbitrarily-shaped scatterers. However, where it is possible to use one of the (semi-)analytical techniques, then these will tend to be faster and more accurate. 



\section{Mie Theory and the $T$-matrix Method}

This chapter provides more details about Mie theory, the $T$-matrix method, and the extended boundary-condition method (EBCM), as a background to methods used in this thesis. Mie theory and the $T$-matrix method are able to treat scattering by both vector or scalar waves, which in practice means either electromagnetic [47] or acoustic [48] scattering respectively. While Mie theory may be considered a special case of the $T$-matrix method (for the case of spheres), it is presented first in line with the historical order of the methods, as well as to present some of the concepts on the simpler Mie theory. Both of these methods have a long history, with Mie theory introduced in 1908 [34, and the $T$-matrix method in 1965 [49]. The following chapters contain improvements to the $T$-matrix method, and represent the progress made in this thesis.

\subsection{Helmholtz Equation in Spherical Coordinates}

The light scattering problem involves solving the Helmholtz equation. Typically light-scattering problems are solved in spherical coordinates, so outgoing waves can

Carrot Cake Preheat oven to $180^{\circ} \mathrm{C}$. Place in a bowl 1.25 cup brown sugar and 0.75 cup vegetable oil. Beat with an electric mixer for 2-3 min. Add 3 eggs gradually, and beat well. Sift in 1.5 cup plain flour, 1.5 tsp baking powder, 1 tsp baking soda, 1 tsp ground cinnamon, and 0.5 tsp ground ginger. Add 2.5 cup grated carrot, 0.5 cup sultanas, and optionally 0.5 cup chopped pecan nuts or walnuts. Pour into a $22 \mathrm{~cm}$ cake tin lined with baking paper. Bake for 55-60 min, or until skewer indicates that it is cooked. Ice when cool with cream-cheese frosting. 


\section{Mie Theory and the T-matrix Method}

be easily viewed as outgoing spherical waves, which is a natural way to view them, especially in the far-field. As we assume that we are solving the problem in a region without sources, we are after divergence-free $(\nabla \cdot \vec{E}=0)$ solutions for $\vec{E}$, and we will solve for $\vec{E}$ only, and realise that $\vec{H}$ may be obtained from this, as discussed in Chapter 2. The Helmholtz equation is, for complex $\vec{E}$,

$$
\nabla^{2} \vec{E}+k^{2} \vec{E}=\overrightarrow{0}
$$

In spherical coordinates, the Laplacian $\left(\nabla^{2}\right)$ operator may be written

$$
\nabla^{2}=\frac{1}{r^{2}} \frac{\partial}{\partial r}\left(r^{2} \frac{\partial}{\partial r}\right)+\frac{1}{r^{2} \sin \theta} \frac{\partial}{\partial \theta}\left(\sin \theta \frac{\partial}{\partial \theta}\right)+\frac{1}{r^{2} \sin ^{2} \theta} \frac{\partial^{2}}{\partial \phi^{2}}
$$

It is possible to show that if some function $f(\vec{r})$ is a solution to the scalar Helmholtz equation, then the field

$$
\vec{M}=\nabla \times(\vec{r} f)
$$

is a solution to the vector Helmholtz equation, and is also divergence-free, as the divergence of a curl is zero [8]. It is also possible to define

$$
\vec{N}=\frac{\nabla \times \vec{M}}{k}
$$

which is also divergence-free and an independent solution to the Helmholtz equation.

In the spherical polar coordinate system, we would like separable solutions, of the form

$$
f(\vec{r})=R(r) \Theta(\theta) \Phi(\phi)
$$

where

$$
\nabla^{2} f+k^{2} f=0
$$


which, by applying Eq. (3.2), leads to

$$
\begin{aligned}
\frac{\mathrm{d}^{2} \Phi}{\mathrm{d} \phi^{2}}+m^{2} \Phi & =0 \\
\frac{1}{\sin \theta} \frac{\mathrm{d}}{\mathrm{d} \theta}\left(\sin \theta \frac{\mathrm{d} \Theta}{\mathrm{d} \theta}\right)+\left[n(n+1)-\frac{m^{2}}{\sin ^{2} \theta}\right] \Theta & =0 \\
\frac{\mathrm{d}}{\mathrm{d} r}\left(r^{2} \frac{\mathrm{d} R}{\mathrm{~d} r}\right)+\left[k^{2} r^{2}-n(n+1)\right] R & =0
\end{aligned}
$$

with separation constants $n$ and $m$. For the first equation, the solutions are sinusoids, $e^{i m \phi}$, and we require that the solution is $2 \pi$-periodic in $\phi$, so $m$ must be an integer. The second equation has solutions that are associated Legendre functions $P_{n}^{m}(\cos \theta)$ [50], as defined in Appendix B. From this, we require that $n$ is an integer, and that $|m| \leq n$. The third equation may be rewritten

$$
x \frac{\mathrm{d}}{\mathrm{d} x}\left(x \frac{\mathrm{d} Z}{\mathrm{~d} x}\right)+\left[x^{2}-\left(n+\frac{1}{2}\right)^{2}\right] Z=0
$$

where $x=k r$ and $Z=\sqrt{x} R$. The solutions to this are spherical Bessel functions of the first kind $j_{n}(k r)$, the second kind $y_{n}(k r)$, and linear combinations of these, the Hankel functions of the first kind $h_{n}^{(1)}(k r)=j_{n}(k r)+i y_{n}(k r)$ and second kind $h_{n}^{(2)}(k r)=j_{n}(k r)-i y_{n}(k r)\left[50\right.$. The solutions that we use are $j_{n}(k r)$ and $h_{n}^{(1)}(k r)$, with the other functions constructable as a linear combination of these. The Hankel function corresponds to outgoing spherical waves, which diverge at the origin, while the Bessel function corresponds to the solution that is finite at the origin. More detail on the form of the Bessel and Hankel functions is provided in Appendix A.

These functions $f$ may give us the electric fields by using Eqs. (3.3) and (3.4). This leads to two different varieties of $\vec{M}$ that we may obtain. These are

$$
\begin{aligned}
& \vec{M}_{n m}^{(1)}(k r, \theta, \phi)=\nabla \times\left(\vec{r} e^{i m \phi} P_{n}^{m}(\cos \theta) j_{n}(k r)\right) \\
& \vec{M}_{n m}^{(3)}(k r, \theta, \phi)=\nabla \times\left(\vec{r} e^{i m \phi} P_{n}^{m}(\cos \theta) h_{n}^{(1)}(k r)\right)
\end{aligned}
$$

where $\vec{M}^{(1)}$ corresponds to regular (finite at the origin) solutions, and $\vec{M}^{(3)}$ corre- 


\section{Mie Theory and the T-matrix Method}

spond to outgoing spherical waves. Following from this, we also have

$$
\begin{aligned}
\vec{N}_{n m}^{(1)}(k r, \theta, \phi) & =\frac{1}{k} \nabla \times \vec{M}^{(1)}(k r, \theta, \phi) \\
\vec{N}_{n m}^{(3)}(k r, \theta, \phi) & =\frac{1}{k} \nabla \times \vec{M}^{(3)}(k r, \theta, \phi) .
\end{aligned}
$$

These functions $\vec{M}$ and $\vec{N}$ are referred to as vector spherical wavefunctions (VSWFs). There are two other varieties, with superscripts ${ }^{(2)}$ and ${ }^{(4)}$, which correspond to the Bessel functions $y_{n}(k r)$ and $h_{n}^{(2)}(k r)$ respectively. These may be constructed by linear combination of the other two VSWFs, as with the Bessel functions. There is more discussion of how to obtain the VSWFs provided in Appendix C.

The use of these VSWFs provides some physical meaning. In particular, we may associate the solutions $\vec{M}_{n m}^{(3)}$ and $\vec{N}_{n m}^{(3)}$ with the fields created by multipoles. Here the $\vec{M}^{(3)}$ solutions correspond to magnetic multipoles at the origin, while the $\vec{N}^{(3)}$ solutions correspond to electric multipoles.

Instead of working with the associated Legendre functions for the angular functions, we instead follow the example of Ref. [9] and work with some related functions, for reasons of numerical stability. We may write a special case of the Wigner $d$-function as

$$
d_{n m}(\theta)=d_{0 m}^{n}(\theta)=(-1)^{m} \sqrt{\frac{(n-m) !}{(n+m) !}} P_{n}^{m}(\cos \theta)
$$

where we make use of the simpler notation $d_{n m}(\theta)$ for simplicity. From this, the functions $\pi_{n m}(\theta)$ and $\tau_{n m}(\theta)$ are defined as

$$
\begin{aligned}
\pi_{n m}(\theta) & =\frac{m d_{n m}(\theta)}{\sin \theta} \\
\tau_{n m}(\theta) & =\frac{\mathrm{d}}{\mathrm{d} \theta} d_{n m}(\theta)
\end{aligned}
$$

More information about these angular functions is provided in Appendix B.

The indices $n$ and $m$ that were introduced into the solutions of $\Phi, \Theta$ and $R$ represent the total angular momentum of the solution, and the projection of the 
angular momentum along the $z$-axis (in our coordinate system), respectively. The values that these indices may take are $0 \leq n<\infty$ and $-n \leq m \leq n$ (which is equivalent to $0 \leq|m| \leq n$ ).

The expressions for $\vec{M}$ and $\vec{N}$ are

$$
\begin{aligned}
& \vec{M}_{n m}(k \vec{r})=(-1)^{m} \sqrt{\frac{(n+m) !}{(n-m) !}} \gamma_{n m} z_{n}(k r) \vec{C}_{n m}(\theta) e^{i m \phi} \\
& \vec{N}_{n m}(k \vec{r})=(-1)^{m} \sqrt{\frac{(n+m) !}{(n-m) !}} \gamma_{n m}\left(\frac{n(n+1)}{k r} z_{n}(k r) \vec{P}_{n m}(\theta)+\frac{z_{n}^{\prime}(k r)}{k r} \vec{B}_{n m}(\theta)\right) e^{i m \phi}
\end{aligned}
$$

where we have the vectors

$$
\begin{aligned}
\vec{B}_{n m}(\theta) & =\hat{\theta} \tau_{n m}(\theta)+\hat{\phi} i \pi_{n m}(\theta) \\
\vec{C}_{n m}(\theta) & =\hat{\theta} i \pi_{n m}(\theta)-\hat{\phi} \tau_{n m}(\theta) \\
\vec{P}_{n m}(\theta) & =\hat{r} d_{n m}(\theta)
\end{aligned}
$$

and

$$
\gamma_{n m}=\sqrt{\frac{(2 n+1)(n-m) !}{4 \pi n(n+1)(n+m) !}} .
$$

The function $z_{n}(k r)$ is one of the spherical Bessel functions, $j_{n}(k r)$ for the regular functions, and $y_{n}(k r)$ for the irregular functions.

\subsection{Plane-Wave Expansion}

Now that we are able to obtain solutions of the Helmholtz equation, we must be able to express the fields we wish to work with, which in general will not be convenient multipolar fields. Depending on the incident field of our situation, the field will be made up of the sum of a series of the individual multipoles. We may express the field in terms of a series with either coefficients $a_{n m}, b_{n m}$ or $c_{n m}, d_{n m}$, associated 


\section{Mie Theory and the T-matrix Method}

with the regular or irregular vector spherical wavefunctions. That is,

$$
\begin{aligned}
& \vec{E}(\vec{r})=\sum_{n=0}^{\infty} \sum_{m=-n}^{n}\left(a_{n m} \vec{M}_{n m}^{(1)}(k \vec{r})+b_{n m} \vec{N}_{n m}^{(1)}(k \vec{r})\right) \\
& \vec{E}(\vec{r})=\sum_{n=0}^{\infty} \sum_{m=-n}^{n}\left(c_{n m} \vec{M}_{n m}^{(3)}(k \vec{r})+d_{n m} \vec{N}_{n m}^{(3)}(k \vec{r})\right)
\end{aligned}
$$

where the choice of which series to use is based on the desired behaviour of the functions (finite at the origin, or behaving as outgoing spherical waves at infinity) in the domain of interest. In the case of the incident wave, the expansion with coefficients $a_{n m}$ and $b_{n m}$ is used. The internal field uses the same series (though with a different set of coefficients, labelled $\alpha_{n m}$ and $\beta_{n m}$ ), and the scattered field makes use of the second series.

It is possible to express any incident wave (within the time-harmonic formalism that underpins all of the work in this thesis) as a series such as that in Eq. (3.24), because the vector spherical wavefunctions form a basis for the divergence-free solutions of the Helmholtz equation. It is possible to calculate the coefficients of the series for any incident wave, and the expressions for plane waves are provided here, without justification. A derivation of this may be found in other sources [8, 9]. The coefficients are

$$
\begin{aligned}
& a_{n m}=4 \pi(-1)^{m} i^{n} d_{n} \vec{E}_{0} \cdot \vec{C}_{n m}^{*}\left(\theta_{p}\right) e^{-i m \phi_{p}} \\
& b_{n m}=4 \pi(-1)^{m} i^{n-1} d_{n} \vec{E}_{0} \cdot \vec{B}_{n m}^{*}\left(\theta_{p}\right) e^{-i m \phi_{p}}
\end{aligned}
$$

where we use the values

$$
\begin{aligned}
d_{n} & =\sqrt{\frac{2 n+1}{4 \pi n(n+1)}} \\
\vec{B}_{n m}\left(\theta_{p}\right) & =\hat{\theta} \tau_{n m}\left(\theta_{p}\right)+\hat{\phi} i \pi_{n m}\left(\theta_{p}\right) \\
\vec{C}_{n m}\left(\theta_{p}\right) & =\hat{\theta} i \pi_{n m}\left(\theta_{p}\right)-\hat{\phi} \tau_{n m}\left(\theta_{p}\right) .
\end{aligned}
$$

In these expressions, the values of $\theta_{p}$ and $\phi_{p}$ correspond to the direction of the incident plane wave. The polarisation information is contained in the vector $\vec{E}_{0}$, 
and $\vec{E}$ and $\overrightarrow{k_{1}}$ are defined as

$$
\begin{aligned}
\vec{E} & =\vec{E}_{0} e^{i \vec{k}_{1} \cdot \vec{r}} \\
\vec{k}_{1} & =k_{x} \hat{x}+k_{y} \hat{y}+k_{z} \hat{z}
\end{aligned}
$$

In the case of incidence along the $\hat{z}$-axis, with the electric field polarised along $\hat{x}$ (so $\left.\theta_{p}=0, \phi_{p}=0\right)$, this may be expressed as

$$
\begin{aligned}
\vec{E} & =\vec{E}_{0} e^{i k_{1} z} \\
\vec{E}_{0} & =E_{0} \hat{x} \\
\vec{B}_{n m}^{*} & =\hat{x} \tau_{n m}(0)-\hat{y} i \pi_{n m}(0) \\
\vec{C}_{n m}^{*} & =-\hat{x} i \pi_{n m}(0)-\hat{y} \tau_{n m}(0),
\end{aligned}
$$

and hence

$$
\begin{aligned}
& \vec{E}_{0} \cdot \vec{B}_{n m}^{*}=E_{0} \tau_{n m}(0) \\
& \vec{E}_{0} \cdot \vec{C}_{n m}^{*}=-i E_{0} \pi_{n m}(0) .
\end{aligned}
$$

For this we note the values of $\pi_{n m}$ and $\tau_{n m}$ at $\theta=0$, which are for $m \neq 0$

$$
\begin{aligned}
\pi_{n m}(0) & =\frac{\sqrt{n(n+1)}}{2} \\
\tau_{n m}(0) & =\frac{m}{n} \sqrt{n(n+1)}
\end{aligned}
$$

and when $m=0$ they are

$$
\begin{aligned}
& \pi_{n 0}(0)=0 \\
& \tau_{n 0}(0)=0 .
\end{aligned}
$$

For this incident field all values of $m$ where $|m| \neq 1$ lead to coefficients of zero, 
3 Mie Theory and the T-matrix Method

and the values of the coefficients $a_{n m}$ and $b_{n m}$ are then

$$
\begin{aligned}
a_{n, 1} & =i^{n+1} \sqrt{\pi(2 n+1)} \\
b_{n, 1} & =i^{n+1} \sqrt{\pi(2 n+1)} \\
a_{n,-1} & =i^{n+1} \sqrt{\pi(2 n+1)} \\
b_{n,-1} & =-i^{n+1} \sqrt{\pi(2 n+1)} .
\end{aligned}
$$

\subsection{Mie Theory}

Mie theory provides a means to relate the coefficients of the incident and scattered fields, as used in the field expansions (3.24) and (3.25). In the case of a sphere, all incident directions are equivalent, and so by choosing the incident direction with an $x$-polarised electric field and with $\vec{k}_{1}$ along $\hat{z}$, only the cases where $|m|=1$ must be considered. There are simple relations between $m=1$ and $m=-1$ terms, and so where possible we will only focus on the $m=1$ terms.

The field expansions that we use for Mie theory are for the incident, scattered and internal fields respectively given by

$$
\begin{aligned}
& \vec{E}_{\mathrm{Inc}}(\vec{r})=\sum_{n=0}^{\infty} \sum_{m=-n}^{n}\left(a_{n m} \vec{M}_{n m}^{(1)}(\vec{r})+b_{n m} \vec{N}_{n m}^{(1)}(\vec{r})\right) \\
& \vec{E}_{\mathrm{Sca}}(\vec{r})=\sum_{n=0}^{\infty} \sum_{m=-n}^{n}\left(c_{n m} \vec{M}_{n m}^{(3)}(\vec{r})+d_{n m} \vec{N}_{n m}^{(3)}(\vec{r})\right) \\
& \vec{E}_{\mathrm{Int}}(\vec{r})=\sum_{n=0}^{\infty} \sum_{m=-n}^{n}\left(\alpha_{n m} \vec{M}_{n m}^{(1)}(\vec{r})+\beta_{n m} \vec{N}_{n m}^{(1)}(\vec{r})\right)
\end{aligned}
$$

where here the external field is obtained as

$$
\vec{E}_{\mathrm{Ext}}=\vec{E}_{\mathrm{Inc}}+\vec{E}_{\mathrm{Sca}} .
$$




\subsubsection{Boundary Conditions}

The starting point for relating the coefficients is to consider the boundary conditions on the particle surface $r=a$. These are

$$
\begin{aligned}
& \left(\vec{E}_{\text {Inc }}+\vec{E}_{\text {Sca }}\right) \cdot \hat{\theta}=\vec{E}_{\text {Int }} \cdot \hat{\theta} \\
& \left(\vec{E}_{\text {Inc }}+\vec{E}_{\text {Sca }}\right) \cdot \hat{\phi}=\vec{E}_{\text {Int }} \cdot \hat{\phi} \\
& \left(\vec{H}_{\text {Inc }}+\vec{H}_{\text {Sca }}\right) \cdot \hat{\theta}=\vec{H}_{\text {Int }} \cdot \hat{\theta} \\
& \left(\vec{H}_{\text {Inc }}+\vec{H}_{\text {Sca }}\right) \cdot \hat{\phi}=\vec{H}_{\text {Int }} \cdot \hat{\phi}
\end{aligned}
$$

which arise from Eqs. (2.29) and (2.30). These may be worked to arrive at

$$
\begin{aligned}
& c_{n m}=\Gamma_{n} a_{n m} \\
& d_{n m}=\Delta_{n} b_{n m}
\end{aligned}
$$

where

$$
\begin{aligned}
\Gamma_{n} & =\frac{s \psi_{n}(x) \psi_{n}^{\prime}(s x)-\psi_{n}(s x) \psi_{n}^{\prime}(x)}{\psi_{n}(s x) \xi_{n}^{\prime}(x)-s \xi_{n}(x) \psi_{n}^{\prime}(s x)} \\
\Delta_{n} & =\frac{\psi_{n}(x) \psi_{n}^{\prime}(s x)-s \psi_{n}(s x) \psi_{n}^{\prime}(x)}{s \psi_{n}(s x) \xi_{n}^{\prime}(x)-\xi_{n}(x) \psi_{n}^{\prime}(s x)}
\end{aligned}
$$

where we make use of the Riccati-Bessel functions $\psi_{n}(x)$ and $\xi_{n}(x)$, as described in Appendix A for convenience, $s$ is the (possibly complex) relative refractive index $s=$ $n_{2} / n_{1}, n_{1}$ and $n_{2}$ are the refractive indices of the medium and particle respectively, and $x$ is the size parameter $x=k_{1} a$. Here $\Gamma_{n}$ relates the coefficients $a_{n m}$ to the coefficients $c_{n m}$, while $\Delta_{n}$ relates $b_{n m}$ to $d_{n m}$.

It is also possible to express the coefficients for the series of the internal field in terms of the coefficients of the incident field, though these are not reproduced here. 


\subsubsection{Scattering Quantities}

The series expansions provide a means of calculating the electric fields at points in space, but these are not the only quantities of interest in light-scattering problems. As seen in Section 2.2.5, there are a selection of cross sections that are of great interest. Within the Mie formalism, these may be analytically computed from the values $\Gamma_{n}$ and $\Delta_{n}$, as sums over all values of $n$. In the case of Mie theory, we are only concerned with cases where $|m|=1$, and there is an easy relation between the coefficients of $m=1$ and $m=-1$.

The expressions for the cross sections are

$$
\begin{aligned}
\sigma_{\mathrm{Sca}} & =\frac{2 \pi}{k_{1}^{2}} \sum_{n=1}^{\infty}(2 n+1)\left(\left|c_{n}\right|^{2}+\left|d_{n}\right|^{2}\right) \\
\sigma_{\mathrm{Ext}} & =\frac{2 \pi}{k_{1}^{2}} \sum_{n=1}^{\infty}(2 n+1) \operatorname{Re}\left(c_{n}+d_{n}\right) \\
\sigma_{\mathrm{Abs}} & =\sigma_{\mathrm{Ext}}-\sigma_{\mathrm{Sca}} .
\end{aligned}
$$

For both series these are only valid for plane-wave excitation, but by symmetry for any incident orientation.

\subsection{T-matrix Principles}

Mie theory works very well for solving the light-scattering problem, with the main drawback being that it is only valid in the case of spheres. However, there is another method that follows a similar approach that works for a much larger class of shapes, the $T$-matrix method. This method was first presented in 1965 by Waterman[49] for the case of perfectly-conducting scatterers, and it was later extended to other cases, such as non-perfect conductors [47], and dielectric scatterers[51]. This method is able to model arbitrary shapes, but some shapes, especially those with sharp edges, may present numerical difficulties. The formulation is similar to that of Mie theory, except now all values of $m$ (with $|m| \leq n$ ) must be considered in general (as not all incident directions are equivalent, we can not always exclude all other $m$-values). 
Now, instead of the field coefficients being related by the vectors $\vec{\Gamma}$ and $\vec{\Delta}$, they are linearly related by the matrix $\mathbf{T}$. That is,

$$
\left(\begin{array}{l}
\vec{c} \\
\vec{d}
\end{array}\right)=\mathbf{T}\left(\begin{array}{l}
\vec{a} \\
\vec{b}
\end{array}\right)
$$

Thus coupling between different orders of $n$ and $m$ is possible, which is not the case in Mie theory. In the case of a sphere, the $T$-matrix method reduces to be the same as Mie theory, in which case the $T$-matrix is diagonal, with the diagonal entries being

$$
\operatorname{diag}(\mathbf{T})=(\vec{\Gamma}, \vec{\Delta})
$$

The coefficients maintain the same meaning in the series expansion of the fields as for Mie theory.

Within the $T$-matrix formalism, the expressions used to calculate desired scattering quantities are similar to Mie theory, and the forms are given here. As for Mie theory, the field is expanded in terms of VSWFs, as

$$
\begin{aligned}
& \vec{E}_{\mathrm{Inc}}(\vec{r})=E_{0} \sum_{n, m}\left(a_{n m} \vec{M}_{n m}^{(1)}\left(k_{1} \vec{r}\right)+b_{n m} \vec{N}_{n m}^{(1)}\left(k_{1} \vec{r}\right)\right) \\
& \vec{E}_{\mathrm{Int}}(\vec{r})=E_{0} \sum_{n, m}\left(\alpha_{n m} \vec{M}_{n m}^{(1)}\left(k_{2} \vec{r}\right)+\beta_{n m} \vec{N}_{n m}^{(1)}\left(k_{2} \vec{r}\right)\right) \\
& \vec{E}_{\mathrm{Sca}}(\vec{r})=E_{0} \sum_{n, m}\left(c_{n m} \vec{M}_{n m}^{(3)}\left(k_{1} \vec{r}\right)+d_{n m} \vec{N}_{n m}^{(3)}\left(k_{1} \vec{r}\right)\right),
\end{aligned}
$$

and as before the external field is calculated as the sum of the incident and scattered fields,

$$
\vec{E}_{\mathrm{Ext}}=\vec{E}_{\mathrm{Inc}}+\vec{E}_{\mathrm{Sca}}
$$

The cross sections are given by 8$]$

$$
\sigma_{\mathrm{Sca}}=\frac{1}{k_{1}^{2}} \sum_{n, m}\left(\left|c_{n m}\right|^{2}+\left|d_{n m}\right|^{2}\right)
$$


3 Mie Theory and the T-matrix Method

$$
\begin{aligned}
\sigma_{\mathrm{Ext}} & =\frac{-1}{k_{1}^{2}} \sum_{n, m}\left(c_{n m}^{*} a_{n m}+d_{n m}^{*} b_{n m}\right) \\
\sigma_{\mathrm{Abs}} & =\sigma_{\mathrm{Ext}}-\sigma_{\mathrm{Sca}} .
\end{aligned}
$$

Additionally, orientation-averaged cross sections may be obtained as 9

$$
\begin{aligned}
\left\langle\sigma_{\text {Sca }}\right\rangle & =\frac{2 \pi}{k_{1}^{2}} \sum_{n, k, m}\left(\left|T_{n k m}^{11}\right|^{2}+\left|T_{n k m}^{12}\right|^{2}+\left|T_{n k m}^{21}\right|^{2}+\left|T_{n k m}^{22}\right|^{2}\right) \\
\left\langle\sigma_{\text {Ext }}\right\rangle & =\frac{-2 \pi}{k_{1}^{2}} \sum_{n, m} \operatorname{Re}\left(T_{n n m}^{11}+T_{n n m}^{22}\right) .
\end{aligned}
$$

where this now depends on entries in $\mathbf{T}$ instead of coefficients of the electric field. This exemplifies one of the main benefits of the $T$-matrix approach: once the $T$ matrix has been calculated, then that matrix may be used to determine the scattering from any incident wave/particle orientation, with only the tasks of calculating the coefficients of the incident wave, and the multiplication in Eq. (3.63) needing to be performed again to obtain the new coefficients of the scattered field. This is in contrast to, for example, the discrete-dipole approximation, where each incident orientation must be separately simulated (though it may be used to calculate the $T$-matrix, this involves simulating many different incident conditions.)

The most common means of calculating the $T$-matrix is that which was provided by Waterman, the extended boundary-condition method (EBCM) [49]. This method is well described in other places [9], and so the full derivation is not provided here. A brief description is provided in the next section.

There are various other methods that may be used to calculate the $T$-matrix. Among those that make use of the EBCM are the iterative EBCM[52], the nullfield method with discrete sources (NFM-DS, null-field method is another term for EBCM) [53]. These approaches are both attempts to extend the practical range of validity of the modelling to higher aspect-ratio particles. The downside of these other methods is that they become more complicated, and lose the benefits of the $T$-matrix method. Taking a different approach than the the EBCM, it is also possible to calculate the $T$-matrix via other methods, such as the discrete-dipole approximation [35, 54], point-matching methods [36] or finite-difference time-domain 
method[55]. However, in order to encode all of the scattering information in the $T$-matrix, it is necessary to model light incident from all directions, which is computationally demanding. These other methods also typically have higher time requirements than the EBCM, and so their benefit arises from the extended range of shapes that they are able to consider.

\subsubsection{Implementation Details}

In the most general case, within the EBCM formalism, $\mathbf{T}$ is obtained as

$$
\mathbf{T}=-\mathbf{P Q}^{-1}
$$

where the matrices $\mathbf{P}$ and $\mathbf{Q}$ will be described shortly. These matrices are structured as block matrices, such as

$$
\mathbf{T}=\left(\begin{array}{ll}
\mathbf{T}^{11} & \mathbf{T}^{12} \\
\mathbf{T}^{21} & \mathbf{T}^{22}
\end{array}\right)
$$

and similarly for the other matrices. Here then we may relate $\mathbf{T}^{11}$ as coupling the $\vec{a}$ and $\vec{c}$ coefficients, $\mathbf{T}^{12}$ as coupling $\vec{a}$ and $\vec{d}, \mathbf{T}^{21}$ as coupling $\vec{b}$ and $\vec{c}$, and $\mathbf{T}^{22}$ as coupling $\vec{b}$ and $\vec{d}$.

Following the formalism of Mishchenko, $\mathbf{P}$ and $\mathbf{Q}$ are calculated through the $\mathbf{J}$ and $\operatorname{Rg} \mathbf{J}$ matrices 1 By using a surface-integral equation, the null-field equation (also called the extended boundary condition), the surface currents may be related to the incident and scattered fields, by expanding the currents and the fields on a basis of VSWFs. The coefficients of these series are related by the matrices $\mathbf{P}$ (for the scattered fields and surface currents) and $\mathbf{Q}$ (for the incident fields and surface currents). These may then be used to related the incident and scattered fields.

\footnotetext{
${ }^{1}$ Here we defer to Mishchenko's notation, and use the Rg prefix to denote that the matrix contains the regular terms, that is those that are finite at the origin. For the most part a different notation will be used, such as $\mathbf{P}$ instead of $\operatorname{Rg} \mathbf{Q}$, for ease of both reading and typing.
} 
These are related by the matrix $\mathbf{Q}$, given explicitly here as

$$
\left(\begin{array}{l}
\vec{a} \\
\vec{b}
\end{array}\right)=\mathrm{Q}\left(\begin{array}{c}
\vec{\alpha} \\
\vec{\beta}
\end{array}\right)
$$

and this is obtained from $\mathbf{J}$ with

$$
\mathbf{Q}=\left(\begin{array}{ll}
\mathbf{Q}^{11} & \mathrm{Q}^{12} \\
\mathbf{Q}^{21} & \mathbf{Q}^{22}
\end{array}\right)=-i k_{1}^{2}\left(\begin{array}{cc}
s \mathbf{J}^{21}+\mathbf{J}^{12} & s \mathbf{J}^{11}+\mathbf{J}^{22} \\
s \mathbf{J}^{22}+\mathbf{J}^{11} & s \mathbf{J}^{12}+\mathbf{J}^{21}
\end{array}\right)
$$

The $J$-matrix is defined as

$$
\begin{aligned}
& J_{n k m m^{\prime}}^{11}=(-1)^{m} \int_{S} \mathrm{~d} S \hat{n} \cdot\left(\vec{M}_{k m^{\prime}}^{(1)}\left(k_{2} r, \theta, \phi\right) \times \vec{M}_{n,-m}^{(3)}\left(k_{1} r, \theta, \phi\right)\right) \\
& J_{n k m m^{\prime}}^{12}=(-1)^{m} \int_{S} \mathrm{~d} S \hat{n} \cdot\left(\vec{M}_{k m^{\prime}}^{(1)}\left(k_{2} r, \theta, \phi\right) \times \vec{N}_{n,-m}^{(3)}\left(k_{1} r, \theta, \phi\right)\right) \\
& J_{n k m m^{\prime}}^{21}=(-1)^{m} \int_{S} \mathrm{~d} S \hat{n} \cdot\left(\vec{N}_{k m^{\prime}}^{(1)}\left(k_{2} r, \theta, \phi\right) \times \vec{M}_{n,-m}^{(3)}\left(k_{1} r, \theta, \phi\right)\right) \\
& J_{n k m m^{\prime}}^{2}=(-1)^{m} \int_{S} \mathrm{~d} S \hat{n} \cdot\left(\vec{N}_{k m^{\prime}}^{(1)}\left(k_{2} r, \theta, \phi\right) \times \vec{N}_{n,-m}^{(3)}\left(k_{1} r, \theta, \phi\right)\right)
\end{aligned}
$$

and the matrix $\operatorname{Rg} \mathbf{J}$ is similar, except that the irregular functions $\vec{M}^{(3)}$ and $\vec{N}^{(3)}$ are replaced by their regular versions, $\vec{M}^{(1)}$ and $\vec{N}^{(1)}$ respectively. Here $k_{1}$ is the wavenumber in the medium, and $k_{2}$ is the wavenumber in the particle.

The matrix $\mathbf{P}$ is calculated in a similar way, except that it makes use of $\operatorname{Rg} \mathbf{J}$ instead of $\mathbf{J}$. $\mathbf{P}$ relates the coefficients of the internal and scattered fields, as

$$
\left(\begin{array}{l}
\vec{c} \\
\vec{d}
\end{array}\right)=-\mathbf{P}\left(\begin{array}{l}
\vec{\alpha} \\
\vec{\beta}
\end{array}\right)
$$

From Eqs. (3.76) and (3.82) we are able to relate the coefficients of the incident and scattered fields. This involves inverting the relationship between the internal and incident fields, and is

$$
\left(\begin{array}{l}
\vec{c} \\
\vec{d}
\end{array}\right)=-\mathbf{P Q}^{-1}\left(\begin{array}{l}
\vec{a} \\
\vec{b}
\end{array}\right)=\mathbf{T}\left(\begin{array}{l}
\vec{a} \\
\vec{b}
\end{array}\right)
$$


which is the canonical $T$-matrix equation.

It is also possible to calculate the internal fields, which depend only on $\mathbf{Q}$. These are calculated as

$$
\left(\begin{array}{l}
\vec{\alpha} \\
\vec{\beta}
\end{array}\right)=\mathbf{R}\left(\begin{array}{l}
\vec{a} \\
\vec{b}
\end{array}\right)
$$

where we have defined $\mathbf{R}=\mathbf{Q}^{-1}$.

We introduce here the matrix $\mathbf{U}$, which is the irregular part of $\mathbf{Q}[7]$. Thus, $\mathbf{Q}$ may be constructed as

$$
\mathbf{Q}=\mathbf{P}+i \mathbf{U}
$$

This follows from the construction of the Riccati-Hankel function $\xi$, which is

$$
\xi_{n}(x)=\psi_{n}(x)+i \chi_{n}(x)
$$

The matrices $\mathbf{P}, \mathbf{Q}$ and $\mathbf{U}$ differ only in the radial functions used, with $\mathbf{P}$ using $\psi_{n}(x) \psi_{k}(s x), \mathbf{Q}$ using $\xi_{n}(x) \psi_{k}(s x)$, and $\mathbf{U}$ using $\chi_{n}(x) \psi_{k}(s x)$. It is common to instead use $\mathbf{Q}$ instead of $\mathbf{U}$, but we have recently suggested that by using $\mathbf{U}$ there are some benefits[7].

\subsubsection{Axisymmetric Particles}

In many cases, particles with some specific symmetry are modelled. The most common case, and that which is the focus of all of the work in this thesis, is axisymmetric particles, which have symmetry of revolution. The convention used in this work is that the particle is rotated about the $z$-axis. Commonly-used particles with this symmetry include spheres, spheroids (which are the focus of much of this thesis), right-circular cylinders, and many others.

There are several simplifications that arise from considering axisymmetric particles. In terms of the $T$-matrix, one of these simplifications is related to the indices $m$ and $m^{\prime}$. For axisymmetric particles, entries of the various matrices are zero unless 


\section{Mie Theory and the T-matrix Method}

$m=m^{\prime}$, and hence in the rest of this thesis, the index $m^{\prime}$ is dropped, and should be considered equal to $m$. A result of this is that the entries for different $m$ s are decoupled. This means that the matrices for different $m$ may be inverted independently, which greatly reduces time and memory requirements. As well as this, the integrals become simplified, from surface integrals to line integrals. The normal to the surface has no $\phi$-component, and the surface element times the unit normal becomes

$$
\begin{aligned}
\mathrm{d} S \hat{n} & =2 \pi r^{2} \sin \theta \mathrm{d} \theta\left(n_{r} \hat{r}+n_{\theta} \hat{\theta}\right) \\
\hat{n} & =n_{r} \hat{r}+n_{\theta} \hat{\theta}
\end{aligned}
$$

where

$$
\begin{aligned}
& n_{r}=\frac{r}{\sqrt{r^{2}+r_{\theta}^{2}}} \\
& n_{\theta}=\frac{-r_{\theta}}{\sqrt{r^{2}+r_{\theta}^{2}}} .
\end{aligned}
$$

One of the most commonly used codes for EBCM calculations of axisymmetric particles is that of Mishchenko[9]. In Mishchenko's formalism, the matrices T, $\mathbf{Q}$ and $\mathbf{P}$, as well as $\mathbf{J}$ and Rg.J, are expressed as block matrices, as in Eq. 3.75. These matrices are the same as those described earlier in this chapter, and the explicit axisymmetric forms of the $J$-matrices are provided in Appendix D.

\subsubsection{Surface Fields}

Often when the fields on the surface are desired, it is more numerically stable to calculate them by using the series expansion for the internal field, and then make use of the boundary conditions to calculate the external surface field. Here we make use of the requirement that the tangential components of the electric field, and the normal components of the electric displacement, are continuous. That is,

$$
\begin{array}{r}
\left(\vec{E}_{-}-\vec{E}_{+}\right) \times \hat{n}=\overrightarrow{0} \\
\left(\varepsilon_{1} \vec{E}_{-}-\varepsilon_{2} \vec{E}_{+}\right) \cdot \hat{n}=0
\end{array}
$$


where $\vec{E}_{-}$is the field immediately inside the particle surface, and $\vec{E}_{+}$is the field immediately outside the surface. Here we have $\hat{n}=n_{r} \hat{r}+n_{\theta} \hat{\theta}$ is the unit-normal vector on the surface (expressions provided in the previous section), and the $\phi$ component is zero when working with axisymmetric particles. This leads to the relations

$$
\begin{aligned}
& E_{r+}=E_{\theta-} n_{r} n_{\theta}\left(s^{2}-1\right)+E_{r-}\left(\left(s^{2}-1\right) n_{r}^{2}+1\right) \\
& E_{\theta+}=E_{\theta-}\left(\left(s^{2}-1\right) n_{\theta}^{2}+1\right)+E_{r-} n_{r} n_{\theta}\left(s^{2}-1\right) \\
& E_{\phi+}=E_{\phi-}
\end{aligned}
$$

where $s$ is the relative refractive index, and so $s^{2}=\varepsilon_{2} / \varepsilon_{1}$.

\subsection{Extensions and Limitations}

The $T$-matrix method, mainly by virtue of its semi-analytical nature, allows several extensions, such as scattering by layered particles [56] or collections of particles [57]. These are briefly explained in the following sections, but for more detail other sources are available[58].

The T-matrix method also suffers from some limitations, which are present both in the EBCM and the T-matrix formalism itself. These are discussed in following sections. For the most part these are numerical problems in calculating $\mathbf{T}$ with the EBCM, though there are also some problems in expanding the field in a VSWF basis in some regions.

\subsubsection{Multiple Scattering}

Multiple scattering in the case of the $T$-matrix method was introduced by Peterson and Ström[57]. Multiple scattering refers to the scattering of light off multiple particles that are close enough that the particles affect the light scattering of the other particles [59]. Widely separated particles may be considered independent scatterers. 


\section{Mie Theory and the T-matrix Method}

The basis for this method is translating the vector spherical wavefunctions used in the expansions of each particle from some global coordinate system (where at least one of the particles is not centred at the origin) to the local coordinate system of each particle. The coefficients of the incident field are re-written for each particle with the appropriate transformation, and then a $T$-matrix for each particle is constructed. It is then possible to calculate the scattered field due to each particle, which includes the effect of scattered light from other particles. From this, it is possible to construct a $T$-matrix for the entire system. This $T$-matrix is treated the same as if it was for only one particle, as it simply relates coefficients of the incident and scattered fields.

The $T$-matrix method has been used to consider collections of large numbers of randomly-oriented particles [60], as well as finding application in considering collections of particles with a varying size or shape distribution [61]. There have also been reports of using the $T$-matrix method to consider the near-field in the case of multiple-particle scattering 62 .

\subsubsection{Layered Scattering}

Layered scattering in the $T$-matrix formalism was introduced by Peterson and Ström[56]. Briefly, this involves expanding the field using a series with both the regular and irregular VSWFs in the layers which do not contain the origin. Then, the $T$-matrix obtained from this system takes into account the internal coupling between the layers, and still represents the coupling between the incident and scattered fields.

\subsubsection{Numerical Problems}

The source of many of the numerical problems has been attributed to inversion of the system of $\mathbf{P}$ and $\mathbf{Q}$ to obtain $\mathbf{T}[6365]$. This is a difficult step for several reasons. Firstly, any inversion will accumulate numerical error, and this can lead to significant errors in the result, even assuming that the matrix being inverted is correct. Secondly, errors in the matrix being inverted will propagate into the inverted system, leading to further errors. Thirdly, the matrices being considered 


\subsection{Extensions and Limitations}

are infinite matrices, but in real-world implementations, they must be truncated to finite sizes. There is some assumption that inverting one of these finite matrices, if it is sufficiently large, will lead to correct values for the entries that exist in that matrix.

One method that is able to counter these numerical problems, with the possible exception of the finite matrix size, is to compute values with increased numerical precision [64]. By using a higher precision to compute values, and truncating the result back to standard double precision for analysis, it is possible to avoid problems associated with numerical integration or matrix inversion, and obtain reliable results. More discussion of this technique is provided in Section 5.2. This approach is useful for studying the behaviour of the method, but owing to the decrease in speed and lack of ease of modifying these codes, actual results are best obtained using other means.

Other errors arise from numerical problems associated with the summation involved in integrating oscillatory functions. More discussion about this is provided in Chapter 5 .

\subsubsection{Fundamental Problems}

Aside from purely numerical problems, there is one main concern with the $T$-matrix method which is much more fundamental in nature, and in this case does not depend on the method used to generate the $T$-matrix. The problem, applies even when the $T$-matrix is known perfectly, and it relates to calculating the near-field. The expansion of the scattered field in terms of outgoing spherical waves centred at the origin is the basis of the $T$-matrix method, yet this expansion is only guaranteed to be valid in the region outside the circumscribing sphere[66, 67]. The assumption that the field may be expressed in terms of this series everywhere is referred to as the 'Rayleigh hypothesis,' and it is commonly accepted that it is not valid everywhere. The effect of this is that for some shapes, it is not possible to calculate a converged value of the field everywhere in the vicinity of the particle, however far-field properties are believed to remain correct. It is worth stressing again that this occurs even when the values in the T-matrix are correct. It is possible to obtain surface fields by 


\section{Mie Theory and the T-matrix Method}

making use of the boundary-conditions of the problem, and using the internal field, but to calculate the fields in the region between the surface, and the region where the series converges, is an outstanding problem[68, 69]. It is worth noting that the region of non-convergence is not typically the entire region inside the circumscribing sphere, but some subset of this [67]. It is also predicted that the field expansion inside the particle fails for some geometries, outside the inscribing sphere, but this is not the case for spheroids, the focus of most of this thesis.

There have been some attempts to improve the behaviour of the $T$-matrix method for calculating near-fields in the case of elongated (high aspect-ratio) particles [70, 71. This uses a range of different sources about which to expand the field, however by using these methods many of the benefits (regarding the analytic nature of the method) are lost.

\subsection{Overview of this Work}

This work is motivated largely by SERS, and so the interest in light scattering is mainly in the area of calculating near- and surface-fields of plasmonic nanoparticles. When using the $T$-matrix method, calculating near-fields typically requires the consideration of higher-order multipoles, with the subsequent increase in numerical error and potential problems with the Rayleigh hypothesis, as compared to calculation of far-field properties. As a result, we are interested in removing sources of numerical error in the $T$-matrix method, which is the focus of the following chapters.

The following two chapters concern modifications made to the EBCM and Tmatrix method. In Chapter 4 are presented some techniques, the first related to the integral forms, and the second to some linear recursion relations between the matrix entries, that while of some value, do not remove all of the numerical problems that trouble the $T$-matrix method. The integral forms allow for simpler manipulation, and remove some small sources of numerical error. These sources of error are not the primary cause of numerical problems, though they do present a simpler implementation. The simpler manipulation was, however, invaluable in working with the linear recursion relations, and the more substantial improvements discussed in Chapter 5. 
The recursion relations, while promising, are an as-yet unfinished work, and require more development to improve calculation of the $T$-matrix.

Chapter 5 presents a more detailed examination of the problems that afflict calculation of the $T$-matrix in the case of spheroids. This chapter demonstrates the cause of the problems (for spheroids and offset spheres), and presents a method of overcoming these in the case of spheroids. The problem turns out to be severe numerical cancellations in the computation of the integrals, which may be removed by modifying the radial (Bessel function) parts of the integrals. By doing this, the integrals may be computed efficiently and without the numerical problems normally encountered. A solution for offset spheres is not provided, or of particular interest, as by using Mie theory and translating the results, this case may be treated without much difficulty.

Chapter 6 uses both the $T$-matrix method, and the finite-element method, to explore near-field light scattering near plasmonic nanoparticles. The $T$-matrix calculations focus on examining the relative distribution (as opposed to absolute intensity) of the field on the surface of particles. 



\section{$4 T$-matrix Formalism and the Extended Boundary-Condition Method}

This chapter begins with a derivation of the new integral expressions used in this work, which provide benefits over more commonly-used expressions [1]. Following that, we develop some linear relations between elements in the $\mathbf{P}$ and $\mathbf{Q}$ matrices. This work, not yet finished, shows that there are linear relations between the matrix elements, and presents expressions for some of these relations. It is hoped that, when completed, this will allow for more efficient calculation of the $T$-matrix.

The expressions for the various $L$ - and $K$-matrices are a product of this thesis, and have been presented in Refs. [1] and [6]. They are derived from (as shown in this chapter) the expressions found in Ref. [9].

\subsection{New Integral Expressions}

We wish to re-write the integral expressions of Mishchenko, given in Appendix D, into a different form, for two main reasons. Firstly, we wish to have simpler expres-

White Chocolate Cake Preheat oven to $160^{\circ} \mathrm{C}$. Put $185 \mathrm{~g}$ butter, 1 cup milk, 1.5 cups white sugar, and $150 \mathrm{~g}$ chopped white chocolate in a saucepan and melt over a low heat, stirring until smooth. Into a bowl add 2 cups sifted plain flour, 1.5 tsp baking powder, 2 eggs and 1 tsp vanilla essence. Add melted mixture and whisk until it is smooth. Pour into a $22 \mathrm{~cm}$ cake tin lined with baking paper. Bake for $50 \mathrm{~min}$, or until a skewer indicates it is cooked. Best iced with a chocolate glaze. 
sions to work with, and secondly our new expressions are separable into a product of functions that depend on the index $n$ and functions that depend on $k$. This allows the $n$ - and $k$-dependent parts to be computed separately, and then the entire matrix of integrands may be computed by appropriate products of the two sets. We shall also see that these simplifications lead to an improvement in the numerical performance of the integrals.

\subsubsection{Simplified Expressions}

We will obtain some different expressions for the integrals. Superficially, we work with the matrix $\mathbf{U}$ instead of $\mathbf{Q}$, which simply involves replacing $\xi_{n}$ by $\chi_{n}[7]$. In addition, by a process of integration by parts, the integrals may be reformulated, and put into a more convenient form[1]. This results in some different integrals. The derivation is presented in the next section, and the results are presented for convenience here:

$$
\begin{aligned}
K_{n k}^{1} & =\int_{0}^{\pi} \mathrm{d} \theta m d_{n} d_{k} x_{\theta} \chi_{n} \psi_{k}^{\prime} \\
K_{n k}^{2} & =\int_{0}^{\pi} \mathrm{d} \theta m d_{n} d_{n} x_{\theta} \chi_{n}^{\prime} \psi_{k} \\
L_{n m}^{1} & =\int_{0}^{\pi} \mathrm{d} \theta \sin \theta x_{\theta} \tau_{n} d_{k} \chi_{n} \psi_{k} \\
L_{n k}^{2} & =\int_{0}^{\pi} \mathrm{d} \theta \sin \theta x_{\theta} d_{n} \tau_{k} \chi_{n} \psi_{k} \\
L_{n k}^{3} & =\int_{0}^{\pi} \mathrm{d} \theta \sin \theta d_{k} \psi_{k}^{\prime}\left[x_{\theta} \tau_{n} \chi_{n}^{\prime}-n(n+1) d_{n} \chi_{n}\right] \\
L_{n k}^{4} & =\int_{0}^{\pi} \mathrm{d} \theta \sin \theta d_{n} \chi_{n}^{\prime}\left[s x_{\theta} \tau_{k} \psi_{k}^{\prime}-k(k+1) d_{k} \psi_{k}\right] \\
L_{n k}^{5} & =n(n+1) L_{n k}^{2}-k(k+1) L_{n k}^{1} \\
& =\int_{0}^{\pi} \mathrm{d} \theta \sin \theta\left[n(n+1) d_{n} \tau_{k}-k(k+1) \tau_{n} d_{k}\right] x_{\theta} \chi_{n} \psi_{k} \\
L_{n k}^{6} & =n(n+1) L_{n k}^{8}-k(k+1) L_{n k}^{7} \\
L_{n k}^{7} & =\int_{0}^{\pi} \mathrm{d} \theta \sin \theta \tau_{n} d_{k} x_{\theta}\left(\chi_{n}^{\prime} \psi_{k}^{\prime}+n(n+1) \frac{\chi_{n} \psi_{k}}{s x^{2}}\right)
\end{aligned}
$$




$$
L_{n k}^{8}=\int_{0}^{\pi} \mathrm{d} \theta \sin \theta d_{n} \tau_{k} x_{\theta}\left(\chi_{n}^{\prime} \psi_{k}^{\prime}+k(k+1) \frac{\chi_{n} \psi_{k}}{s x^{2}}\right)
$$

These integrals take the two different labels $L$ and $K$, with the distinction between them being in the parity they take in the case of particles with a plane of symmetry. The $L$-matrices have the same parity requirements as the diagonal blocks $\mathbf{T}^{11}$ and $\mathbf{T}^{22}$, which is that for particles with a plane of symmetry the values are zero when $n+k$ is odd. The $K$-matrices share their parity requirements with $\mathbf{T}^{12}$ and $\mathbf{T}^{21}$, which is that for such particles the entries are zero when $n+k$ is even. Hence the $L$-matrices are involved in the diagonal blocks of $\mathbf{P}$ and $\mathbf{U}$, while the $K$-matrices are used in the off-diagonal blocks.

From these $K$ - and $L$-integrals, we are able to construct the $U$-matrix as

$$
\begin{aligned}
U_{n k}^{11} & =\frac{i a_{n} a_{k}\left(s^{2}-1\right) / s}{n(n+1)-k(k+1)} L_{n k}^{5} \\
U_{n k}^{12} & =a_{n} a_{k} \frac{s^{2}-1}{s} K_{n k}^{1} \\
U_{n k}^{21} & =a_{n} a_{k} \frac{1-s^{2}}{s} K_{n k}^{2} \\
U_{n k}^{22} & =\frac{i a_{n} a_{k}\left(s^{2}-1\right) / s}{n(n+1)-k(k+1)} L_{n k}^{6}
\end{aligned}
$$

where the expressions for $\mathbf{U}^{11}$ and $\mathbf{U}^{22}$ are valid only for entries not on the diagonal $(n \neq k)$. For those entries, we use the expressions

$$
\begin{aligned}
& U_{n k}^{11}=i a_{n} a_{k}\left(s L_{n k}^{1}-L_{n k}^{3}-\frac{L_{n k}^{2}-L_{n k}^{4}}{s}\right) \\
& U_{n k}^{22}=i a_{n} a_{k}\left(-L_{n k}^{2}+L_{n k}^{4}-L_{n k}^{1}-\frac{L_{n k}^{3}}{s}\right),
\end{aligned}
$$

which are actually valid for all entries. As before, to calculate the regular matrix $(\mathbf{P})$, we perform the same calculations, but we replace the irregular Bessel functions $\chi_{n}(x)$ with the regular Bessel functions $\psi_{n}(x)$. Using this notation, $\mathbf{T}$ is calculated as

$$
\mathbf{T}=-\mathbf{P}(\mathbf{P}+i \mathbf{U})^{-1}=-\mathbf{P Q}^{-1}
$$




\section{T-matrix Formalism and the Extended Boundary-Condition Method}

In order to derive these expressions, we start from the expressions of Mishchenko [9], Eqs. (D.5)-(D.8) (see Appendix D for the expressions). We also make use of some properties of the angular and radial functions, in order to allow some simplifications.

In all of the following, we consider elements that form the matrix $\mathbf{U}$. Clearly the matrix $\mathbf{P}$ must also be calculated, but as the results for $\mathbf{P}$ ( or $\mathbf{Q}$ ) may be trivially derived from those of $\mathbf{U}$, they are not presented separately. In order to transform these expressions into ones applicable to $\mathbf{P}$, simply replace all instances of $\chi_{n}(x)$ with $\psi_{n}(x)$. As all of the relations on these functions are simply based on the differential equation which they both obey, they may be interchanged and all the results will remain unchanged.

\subsubsection{Useful Relations}

We provide here some relations that are useful for the derivation, both involving the angular functions, and involving the radial functions.

The derivation of the relations between the various angular functions is not provided here. Instead, it is included in Appendix B. Here are provided the final results for convenience, using the reduced notation omitting the index $m$ and the argument $\theta$ of most functions.

$$
\begin{aligned}
{\left[\pi_{n} \tau_{k}+\tau_{n} \pi_{k}\right] \sin \theta } & =m\left(d_{n} d_{k}\right)^{\prime} \\
{\left[\pi_{n} \pi_{k}+\tau_{n} \tau_{k}\right] \sin \theta } & =\left(d_{n} \tau_{k} \sin \theta\right)^{\prime}+k(k+1) d_{n} d_{k} \sin \theta \\
{\left[\pi_{n} \pi_{k}+\tau_{n} \tau_{k}\right] \sin \theta } & =\left(\tau_{n} d_{k} \sin \theta\right)^{\prime}+n(n+1) d_{n} d_{k} \sin \theta \\
\left(\tau_{n} d_{k} \sin \theta\right)^{\prime} & =\sin \theta\left(\tau_{n} \tau_{k}+\pi_{n} \pi_{k}-n(n+1) d_{n} d_{k}\right) \\
\left(d_{n} \tau_{k} \sin \theta\right)^{\prime} & =\sin \theta\left(\tau_{n} \tau_{k}+\pi_{n} \pi_{k}-k(k+1) d_{n} d_{k}\right)
\end{aligned}
$$

There are also relations between the radial (Bessel) functions, which must be understood in order to simplify the integrals. The differential equation which defines the Riccati-Bessel functions is ([50], Eq. (10.3.1))

$$
x^{2} w^{\prime \prime}+\left[x^{2}-n(n+1)\right] w=0
$$


where $n$ is an integer, and $w(x)$ is one of the Riccati-Bessel functions. This allows us to express the second derivative of these functions in terms of just the function, its argument and its order, as (by simple rearranging)

$$
w^{\prime \prime}=w\left(\frac{n(n+1)}{x^{2}}-1\right)
$$

The two functions that we are primarily concerned with are $\psi_{k} \equiv \psi_{k}(s x)=s x j_{k}(s x)$ and $\chi_{n} \equiv \chi_{n}(x)=x y_{n}(x)$, given here in terms of the spherical Bessel functions of the first and second kinds, $j$ and $y$ respectively.

It is possible to write an expression between some differentiated and non-differentiated terms, using simple differentiation, as

$$
\begin{aligned}
{\left[n(n+1) s^{2}-k(k+1)\right] x_{\theta} \chi_{n} \psi_{k}=} & \frac{\mathrm{d}}{\mathrm{d} \theta}\left[k(k+1) \chi_{n}^{\prime} \psi_{k}-n(n+1) s \chi_{n} \psi_{k}^{\prime}\right] \\
& +[n(n+1)-k(k+1)] s x_{\theta} \chi_{n}^{\prime} \psi_{k}^{\prime} .
\end{aligned}
$$

\subsubsection{Derivation of $\mathrm{U}^{12}$ and $\mathrm{U}^{21}$}

Here we present the derivation of the expressions for the off-diagonal blocks $\mathbf{U}^{12}$ and $\mathbf{U}^{21}$. Starting with $\mathbf{U}^{12}$, we may combine the expressions for $\mathbf{J}^{11}$ and $\mathbf{J}^{22}$ to arrive at

$$
\begin{aligned}
U_{n k}^{12}= & -i k_{1}^{2}\left(s J_{n k}^{11}+J_{n k}^{22}\right) \\
= & -a_{n} a_{k} \int_{0}^{\pi} \mathrm{d} \theta \sin \theta\left[\left(\chi_{n} \psi_{k}+\frac{\chi_{n}^{\prime} \psi_{k}^{\prime}}{s}\right)\left[\pi_{n} \tau_{k}+\tau_{n} \pi_{k}\right]\right. \\
& \left.+\left(n(n+1) \chi_{n} \psi_{k}^{\prime}+\frac{k(k+1)}{s} \chi_{n}^{\prime} \psi_{k}\right) \frac{x_{\theta}}{s x^{2}} \pi_{n} d_{k}\right] .
\end{aligned}
$$


Making use of Eq. 4.18), this may be rewritten as

$$
\begin{aligned}
\frac{-U_{n k}^{12}}{a_{n} a_{k}}= & \int_{0}^{\pi} \mathrm{d} \theta m\left[\left(\chi_{n} \psi_{k}+\frac{\chi_{n}^{\prime} \psi_{k}^{\prime}}{s}\right)\left(d_{n} d_{k}\right)^{\prime}\right. \\
& \left.+\left(n(n+1) \chi_{n} \psi_{k}^{\prime}+\frac{k(k+1)}{s} \chi_{n}^{\prime} \psi_{k}\right) \frac{x_{\theta}}{s x^{2}} d_{n} d_{k}\right]
\end{aligned}
$$

We then can see that, using integration by parts,

$$
\begin{aligned}
\int_{0}^{\pi} \mathrm{d} \theta m\left(d_{n} d_{k}\right)^{\prime} & \left(\chi_{n} \psi_{k}+\frac{\chi_{n}^{\prime} \psi_{k}^{\prime}}{s}\right)=\left.m d_{n} d_{k}\left(\chi_{n} \psi_{k}+\frac{\chi_{n}^{\prime} \psi_{k}^{\prime}}{s}\right)\right|_{0} ^{\pi} \\
& -\int_{0}^{\pi} \mathrm{d} \theta m d_{n} d_{k} x_{\theta}\left(\chi_{n}^{\prime} \psi_{k}+s \chi_{n} \psi_{k}^{\prime}+\frac{\chi_{n}^{\prime \prime} \psi_{k}^{\prime}}{s}+\chi_{n}^{\prime} \psi_{k}^{\prime \prime}\right)
\end{aligned}
$$

and using the fact that $d(\theta=0)=d(\theta=\pi)=0$, we exclude the first term. By also making use of Eq. (4.24), we then have

$$
\begin{aligned}
\int_{0}^{\pi} \mathrm{d} \theta m\left(d_{n} d_{k}\right)^{\prime} & \left(\chi_{n} \psi_{k}+\frac{\chi_{n}^{\prime} \psi_{k}^{\prime}}{s}\right)= \\
& -\int_{0}^{\pi} \mathrm{d} \theta m d_{n} d_{k} x_{\theta}\left(\chi_{n}^{\prime} \psi_{k}+s \chi_{n} \psi_{k}^{\prime}+\frac{\chi_{n}^{\prime \prime} \psi_{k}^{\prime}}{s}+\chi_{n}^{\prime} \psi_{k}^{\prime \prime}\right) \\
= & -\int_{0}^{\pi} \mathrm{d} \theta m d_{n} d_{k} x_{\theta}\left(\chi_{n} \psi_{k}^{\prime}\left(\frac{n(n+1)}{s x^{2}}-\frac{1}{s}+s\right)+\chi_{n}^{\prime} \psi_{k} \frac{k(k+1)}{s^{2} x^{2}}\right)
\end{aligned}
$$

Substituting this back in to our expression for $U_{n k}^{12}$, Eq. (4.26), several of the terms cancel with each other, leaving the simpler expression

$$
\frac{-U_{n k}^{12}}{a_{n} a_{k}}=\int_{0}^{\pi} \mathrm{d} \theta m d_{n} d_{k} x_{\theta} \chi_{n} \psi_{k}^{\prime}\left(\frac{1}{s}-s\right)
$$

and hence finally

$$
U_{n k}^{12}=a_{n} a_{k} \frac{s^{2}-1}{s} \int_{0}^{\pi} \mathrm{d} \theta m d_{n} d_{k} x_{\theta} \chi_{n} \psi_{k}^{\prime} .
$$

The derivation of $\mathbf{U}^{21}$ follows similar lines, as the only difference in the definition 
is that the positions of $\mathbf{J}^{11}$ and $\mathbf{J}^{22}$ are interchanged. We start again from the definition of $\mathbf{U}^{21}$,

$$
\begin{aligned}
\frac{U_{n k}^{21}}{a_{n} a_{k}}= & -\int_{0}^{\pi} \mathrm{d} \theta \sin \theta\left[\left(\frac{\chi_{n} \psi_{k}}{s}+\chi_{n}^{\prime} \psi_{k}^{\prime}\right)\left[\pi_{n} \tau_{k}+\tau_{n} \pi_{k}\right]\right. \\
& \left.+\left(n(n+1) \chi_{n} \psi_{k}^{\prime}+\frac{k(k+1)}{s} \chi_{n}^{\prime} \psi_{k}\right) \frac{x_{\theta}}{x^{2}} \pi_{n} d_{k}\right]
\end{aligned}
$$

As before, we may use Eq. 4.18) in order to simplify things, giving

$$
\begin{aligned}
\frac{U_{n k}^{21}}{a_{n} a_{k}}= & -\int_{0}^{\pi} \mathrm{d} \theta m\left[\left(\frac{\chi_{n} \psi_{k}}{s}+\chi_{n}^{\prime} \psi_{k}^{\prime}\right)\left(d_{n} d_{k}\right)^{\prime}\right. \\
& \left.+\left(n(n+1) \chi_{n} \psi_{k}^{\prime}+\frac{k(k+1)}{s} \chi_{n}^{\prime} \psi_{k}\right) \frac{x_{\theta}}{x^{2}} d_{n} d_{k}\right] .
\end{aligned}
$$

Again, this may be modified using integration by parts, using

$$
\int_{0}^{\pi} \mathrm{d} \theta m\left(\frac{\chi_{n} \psi_{k}}{s}+\chi_{n}^{\prime} \psi_{k}^{\prime}\right)\left(d_{n} d_{k}\right)^{\prime}=-\int_{0}^{\pi} \mathrm{d} \theta m d_{n} d_{k} x_{\theta}\left(\frac{\chi_{n}^{\prime} \psi_{k}}{s}+\chi_{n} \psi_{k}^{\prime}+\chi_{n}^{\prime \prime} \psi_{k}^{\prime}+s \chi_{n}^{\prime} \psi_{k}^{\prime \prime}\right),
$$

and by substituting in the expressions for the second derivatives, Eq. 4.24, we obtain

$$
\begin{aligned}
\int_{0}^{\pi} \mathrm{d} \theta m & \left(\frac{\chi_{n} \psi_{k}}{s}+\chi_{n}^{\prime} \psi_{k}^{\prime}\right)\left(d_{n} d_{k}\right)^{\prime}= \\
& -\int_{0}^{\pi} \mathrm{d} \theta m d_{n} d_{k} x_{\theta}\left(\frac{n(n+1)}{x^{2}} \chi_{n} \psi_{k}^{\prime}+\chi_{n}^{\prime} \psi_{k}\left(\frac{k(k+1)}{s x^{2}}-s+\frac{1}{s}\right)\right) .
\end{aligned}
$$

Placing this back into Eq. 4.34, we end up with the expression

$$
U_{n k}^{21}=a_{n} a_{k} \frac{1-s^{2}}{s} \int_{0}^{\pi} \mathrm{d} \theta m d_{n} d_{k} x_{\theta} \chi_{n}^{\prime} \psi_{k},
$$

which has the same form as that for $\mathbf{U}^{12}$, differing only in sign and which of the Bessel functions is differentiated. 


\subsubsection{Derivation of $\mathrm{U}^{11}$ and $\mathrm{U}^{22}$}

The derivation of alternate forms for the diagonal blocks of $\mathbf{U}$ does not yield expressions that are as simple as those of the off-diagonal blocks. However, the expressions used do offer some advantages over the expressions of Mishchenko, which will be discussed later.

Here will be shown the derivation of the integral expressions, first in the general case which is valid for all entries, and then in the case valid only for off-diagonal entries.

We start here by simple looking at $\mathbf{J}^{12}$. Firstly, we note that by using Eq. 4.19, and also making use of Eq. (4.24), and realising that $d_{n}(\theta=0)=d_{n}(\theta=\pi)=0$, we may write part of the expression of $\mathbf{J}^{12}$ as

$$
\begin{aligned}
\int_{0}^{\pi} \mathrm{d} \theta \sin \theta \chi_{n}^{\prime} \psi_{k}\left[\pi_{n} \pi_{k}+\tau_{n} \tau_{k}\right]= & \int_{0}^{\pi} \mathrm{d} \theta \chi_{n}^{\prime} \psi_{k}\left[\left(d_{n} \tau_{k} \sin \theta\right)^{\prime}+k(k+1) \sin \theta d_{n} d_{k}\right] \\
= & \int_{0}^{\pi} \mathrm{d} \theta \sin \theta\left[k(k+1) d_{n} d_{k} \chi_{n}^{\prime} \psi_{k}\right. \\
& \left.-d_{n} \tau_{k} x_{\theta}\left(s \chi_{n}^{\prime} \psi_{k}^{\prime}+\left(\frac{n(n+1)}{x^{2}}-1\right) \chi_{n} \psi_{k}\right)\right],
\end{aligned}
$$

and by including the other term from $\mathbf{J}^{12}$, then we end up with

$$
\begin{aligned}
\frac{s k_{1}^{2} J_{n k}^{12}}{a_{n} a_{k}} & =\int_{0}^{\pi} \mathrm{d} \theta \sin \theta\left(k(k+1) d_{n} d_{k} \chi_{n}^{\prime} \psi_{k}+x_{\theta} d_{n} \tau_{k} \chi_{n} \psi_{k}-s x_{\theta} d_{n} \tau_{k} \chi_{n}^{\prime} \psi_{k}^{\prime}\right) \\
& =L_{n k}^{2}-L_{n k}^{4}
\end{aligned}
$$

where $L_{n k}^{2}$ and $L_{n k}^{4}$ are as defined in Eqs. (4.4) and (4.6) respectively. 
We may do something similar to $\mathbf{J}^{21}$, this time using Eq. 4.20), to see that

$$
\begin{aligned}
\int_{0}^{\pi} \mathrm{d} \theta \sin \theta \chi_{n} \psi_{k}^{\prime}\left[\pi_{n} \pi_{k}+\tau_{n} \tau_{k}\right]= & \int_{0}^{\pi} \mathrm{d} \theta \chi_{n} \psi_{k}^{\prime}\left[\left(\tau_{n} d_{k} \sin \theta\right)^{\prime}+n(n+1) \sin \theta d_{n} d_{k}\right] \\
= & \int_{0}^{\pi} \mathrm{d} \theta \sin \theta\left[n(n+1) d_{n} d_{k} \chi_{n} \psi_{k}^{\prime}\right. \\
& \left.-\tau_{n} d_{k} x_{\theta}\left(\chi_{n}^{\prime} \psi_{k}^{\prime}+\left(\frac{k(k+1)}{s x^{2}}-s\right) \chi_{n} \psi_{k}\right)\right]
\end{aligned}
$$

and combining with the other part of the expression for $\mathbf{J}^{21}$ we are left with

$$
\begin{aligned}
\frac{-s k_{1}^{2} J_{n k}^{21}}{a_{n} a_{k}} & =\int_{0}^{\pi} \mathrm{d} \theta \sin \theta\left(n(n+1) d_{n} d_{k} \chi_{n} \psi_{k}^{\prime}+s x_{\theta} \tau_{n} d_{k} \chi_{n} \psi_{k}-x_{\theta} \tau_{n} d_{k} \chi_{n}^{\prime} \psi_{k}^{\prime}\right) \\
& =s L_{n k}^{1}-L_{n k}^{3}
\end{aligned}
$$

where $L_{n k}^{1}$ and $L_{n k}^{3}$ are as defined in Eqs. 4.3 and 4.5 respectively.

From these expressions for $\mathbf{J}^{12}$ and $\mathbf{J}^{21}$, it is now easy to write expressions for $\mathbf{U}^{11}$ and $\mathbf{U}^{22}$. These are

$$
\begin{aligned}
& U_{n k}^{11}=i a_{n} a_{k}\left(s L_{n k}^{1}-L_{n k}^{3}-\frac{L_{n k}^{2}-L_{n k}^{4}}{s}\right) \\
& U_{n k}^{22}=i a_{n} a_{k}\left(-L_{n k}^{2}+L_{n k}^{4}-L_{n k}^{1}-\frac{L_{n k}^{3}}{s}\right) .
\end{aligned}
$$

These expressions are valid for all matrix entries. However, other expressions are preferred over these, as they require calculating fewer integrals, and have slightly improved numerical performance. 
4 T-matrix Formalism and the Extended Boundary-Condition Method

In the case of off-diagonal elements, then we make use of Eq. 4.25 to see that

$$
\begin{aligned}
L_{n k}^{1}-L_{n k}^{2}= & \int_{0}^{\pi} \mathrm{d} \theta \sin \theta x_{\theta} \chi_{n} \psi_{k}\left(\tau_{n} d_{k}-d_{n} \tau_{k}\right) \\
= & \int_{0}^{\pi} \frac{\mathrm{d} \theta \sin \theta\left(\tau_{n} d_{k}-d_{n} \tau_{k}\right)}{n(n+1) s^{2}-k(k+1)}\left[\left(k(k+1) \chi_{n}^{\prime} \psi_{k}-s n(n+1) \chi_{n} \psi_{k}^{\prime}\right)^{\prime}\right. \\
& \left.+s(n(n+1)-k(k+1)) x_{\theta} \chi_{n}^{\prime} \psi_{k}^{\prime}\right] \\
= & \int_{0}^{\pi} \frac{\mathrm{d} \theta \sin \theta(n(n+1)-k(k+1))}{n(n+1) s^{2}-k(k+1)}\left\{s \tau_{n} d_{k} \chi_{n}^{\prime} \psi_{k}^{\prime}\right. \\
& +s x_{\theta} n(n+1) d_{n} d_{k} \chi_{n} \psi_{k}^{\prime}-x_{\theta} k(k+1) d_{n} d_{k} \chi_{n}^{\prime} \psi_{k} \\
& \left.-s(n(n+1)-k(k+1)) d_{n} \tau_{k} \chi_{n}^{\prime} \psi_{k}^{\prime}\right\} \\
= & \frac{n(n+1)-k(k+1)}{n(n+1) s^{2}-k(k+1)}\left[s L_{n k}^{3}-L_{n k}^{4}\right] .
\end{aligned}
$$

Using this relation, we are able to write the expressions for $\mathbf{U}^{11}$ and $\mathbf{U}^{22}$ as

$$
\begin{aligned}
U_{n k}^{11} & =i a_{n} a_{k}\left(s L_{n k}^{1}-\frac{L_{n k}^{2}}{s}-L_{n k}^{3}+\frac{L_{n k}^{4}}{s}\right) \\
& =i a_{n} a_{k}\left(s L_{n k}^{1}-\frac{L_{n k}^{2}}{s}-\frac{L_{n k}^{1}-L_{n k}^{2}}{s} \times \frac{n(n+1) s^{2}-k(k+1)}{n(n+1)-k(k+1)}\right) \\
& =\frac{i a_{n} a_{k}\left(s^{2}-1\right) / s}{n(n+1)-k(k+1)}\left(n(n+1) L_{n k}^{2}-k(k+1) L_{n k}^{1}\right)
\end{aligned}
$$

and

$$
\begin{aligned}
U_{n k}^{22} & =i a_{n} a_{k}\left(L_{n k}^{1}-L_{n k}^{2}-\left(\frac{L_{n k}^{3}}{s}-L_{n k}^{4}\right)\right) \\
& =i a_{n} a_{k}\left(L_{n k}^{1}-L_{n k}^{2}+\frac{s^{2}-1}{s} L_{n k}^{3}-\left(\frac{n(n+1) s^{2}-k(k+1)}{n(n+1)-k(k+1)}\right)\left(L_{n k}^{1}-L_{n k}^{3}\right)\right) \\
& =i a_{n} a_{k}\left(s^{2}-1\right) / s\left(L_{n k}^{3}+\frac{n(n+1) s\left(L_{n k}^{1}-L_{n k}^{2}\right)}{n(n+1)-k(k+1)}\right)
\end{aligned}
$$


From these expressions, we define more integrals

$$
\begin{aligned}
L_{n k}^{5} & =n(n+1) L_{n k}^{2}-k(k+1) L_{n k}^{1} \\
& =\int_{0}^{\pi} \mathrm{d} \theta \sin \theta\left[n(n+1) d_{n} \tau_{k}-k(k+1) \tau_{n} d_{k}\right] x_{\theta} \chi_{n} \psi_{k} \\
L_{n k}^{6} & =n(n+1) L_{n k}^{8}-k(k+1) L_{n k}^{7}
\end{aligned}
$$

where

$$
\begin{aligned}
& L_{n k}^{7}=\int_{0}^{\pi} \mathrm{d} \theta \sin \theta \tau_{n} d_{k} x_{\theta}\left(\chi_{n}^{\prime} \psi_{k}^{\prime}+n(n+1) \frac{\chi_{n} \psi_{k}}{s x^{2}}\right) \\
& L_{n k}^{8}=\int_{0}^{\pi} \mathrm{d} \theta \sin \theta d_{n} \tau_{k} x_{\theta}\left(\chi_{n}^{\prime} \psi_{k}^{\prime}+k(k+1) \frac{\chi_{n} \psi_{k}}{s x^{2}}\right)
\end{aligned}
$$

These expressions, valid only when $n \neq k$ (that is the off-diagonal elements), eliminate the need to calculate $L_{n k}^{4}$, and also reduce the effect of numerical cancellations. The expressions for the off-diagonal elements of $\mathbf{U}^{11}$ and $\mathbf{U}^{22}$ are now

$$
\begin{aligned}
U_{n k}^{11} & =\frac{i a_{n} a_{k}\left(s^{2}-1\right) / s}{n(n+1)-k(k+1)} L_{n k}^{5} \\
U_{n k}^{22} & =\frac{i a_{n} a_{k}\left(s^{2}-1\right) / s}{n(n+1)-k(k+1)} L_{n k}^{6} .
\end{aligned}
$$

It is also possible to re-write the expressions Eqs. 4.42 and 4.43 in a valid form for the diagonal elements, as

$$
\begin{aligned}
& U_{n n}^{11}=\tilde{L}_{n}^{1} \\
& U_{n n}^{22}=\tilde{L}_{n}^{2}+\tilde{L}_{n}^{3}
\end{aligned}
$$

where

$$
\begin{aligned}
& \tilde{L}_{n}^{1}=-\frac{i a_{n}^{2}}{s} \int_{0}^{\pi} \mathrm{d} \theta \sin \theta\left(\pi_{n} \pi_{n}+\tau_{n} \tau_{n}\right)\left(\chi_{n}^{\prime} \psi_{n}-s \chi_{n} \psi_{n}^{\prime}\right) \\
& \tilde{L}_{n}^{2}=-\frac{i a_{n}^{2}}{s} \int_{0}^{\pi} \mathrm{d} \theta \sin \theta\left(\pi_{n} \pi_{n}+\tau_{n} \tau_{n}\right)\left(s \chi_{n}^{\prime} \psi_{n}-\chi_{n} \psi_{n}^{\prime}\right)
\end{aligned}
$$


4 T-matrix Formalism and the Extended Boundary-Condition Method

$$
\tilde{L}_{n}^{3}=-\frac{i a_{n}^{2} n(n+1)\left(s^{2}-1\right)}{s} \int_{0}^{\pi} \mathrm{d} \theta \sin \theta x_{\theta} d_{n} \tau_{n} \frac{\chi_{n} \psi_{n}}{s x^{2}}
$$

These are trivially arrived at by application of Eq. (3.77), using the expressions (D.6) and (D.7), for the case where $n=k$.

\subsubsection{Benefits of the New Expressions}

Now that we have arrived at these new expressions, one obvious question is to ask if they provide any benefits over the starting expressions. One benefit is that they enable us to separate the $n$ - and $k$-dependent parts of the integrals. This allows the two parts to be calculated separately, and then easily combined to populate the entire matrix, which can lead to speed gains. The main benefit, however, comes from the reduction in the number of cancellations from combining the integrals. This is most apparent for the off-diagonal blocks, which are the expressions which simplified the most. In the case of a cylinder, with refractive index $n_{2}=0.09+4 i$ (corresponding to silver) in water $\left(n_{1}=1.33\right)$, with a radius of $20 \mathrm{~nm}$ and height $80 \mathrm{~nm}$, at an incident wavelength of $633 \mathrm{~nm}$, we compared the matrix $\mathbf{Q}^{12}$ for $m=1$ when calculated with the double-precision code of Mishchenko[9] and these new expressions, and compared these matrices to one calculated using arbitrary-precision code. It was found that the old expressions agreed within an order of magnitude up to $N=35$, while with our new expressions, this agreement was obtained to $N=43$.

In addition, these expressions look simpler, and hence lend themselves more to analytical manipulation. This is exploited in the second half of this chapter, and in Chapter 5 .

\subsubsection{Summary of which Expressions are Used}

The various codes that we use include an arbitrary-precision $\mathrm{C}$ implementation, a double-precision MATLAB implementation, and a double-precision MATLAB implementation for spheroids, which has some improvements for the special case of spheroids. The two former codes both predate this thesis, but have been modified 
slightly for performance and numerical reasons during this thesis.

All of the codes calculate $\mathbf{Q}$ directly, and do not calculate $\mathbf{U}$, however this simply involves replacing the values $\chi_{n}$ used in the previous derivation with the values $\xi_{n}=\psi_{n}+i \chi_{n}$.

In general, the arbitrary-precision code may use forms that suffer from cancellations, as we are able to increase the working precision to remove the problem of loss of precision. Thus, it makes use of integrals given in Eqs. 4.42 and (4.43) to calculate all of the matrices $\mathbf{Q}^{11}$ and $\mathbf{Q}^{22}$. These are also the forms used in the original double-precision MATLAB code, as the improved expressions (Eqs. 4.45) and (4.46) ) were developed after that code.

The new spheroid-only MATLAB code uses the expressions given in Eqs. (4.32), (4.37), 4.45, 4.46), 4.53 and 4.54), but with an important modification, which is explained more fully in Chapter 5. In short, for some parts of the matrix $\mathbf{Q}$ (but not $\mathbf{P}$ ) this replaces the products $\xi_{n} \psi_{k}, \xi_{n}^{\prime} \psi_{k}$ and so on with modified products that lead to improved numerical behaviour the integrals are calculated. Also, on the diagonal of $\mathbf{P}^{11}$, there is an additional cancellation which is rectified by using some different expressions in that case. This is discussed more in Section 5.6.5

Owing to reasons of parity, in the case of shapes with a plane of symmetry at $z=0$, then half of the entries in $\mathbf{Q}$ are zero (and similarly in all of the other matrices we use). For the diagonal blocks $\mathbf{Q}^{11}$ and $\mathbf{Q}^{22}$ the entries that are zero are those for which $n+k$ is odd, and for the off-diagonal blocks the entries that are zero have $n+k$ even. Based on the integral forms that are used in the case of spheroids, this parity enables us to calculate only the terms that are actually required, and not to calculate any off-parity terms.

\subsection{T-matrix Recursion Relations}

One of the computational limitations in the EBCM is that as higher-order multipoles (up to order $N$ ) are required, the number of integrals that must be calculated increases as $N^{3}$ (assuming all $m$ s are required). As well as this computational ex- 


\section{T-matrix Formalism and the Extended Boundary-Condition Method}

pense, performing the integrals inevitably leads to a loss of precision, aside from that experienced for certain shapes (see Chapter 5). One way of overcoming this loss of precision is to work in a higher precision, such that the results remain valid in (typically) double precision. However, such code is much slower than double precision code (see comments in Section 5.6.6 for an example). As such, another approach is sought.

One possible such approach is to find simple relations between the matrix elements, such that the number of elements that are required to be computed is reduced. Here 'simple' means computationally simple, for example linear relations. Here we look for relations in $\mathbf{P}$ and $\mathbf{U}$, as we have expressions for these integrals and so can hope to find (and prove) relations between elements.

The work presented in the following sections is as-yet unfinished, and as a result not every case has been considered. Specifically, the relations for all $m$ have not been found, and as a result, it is not yet clear if this method might provide much benefit. However, the results to date are provided here, and it is hoped that more will be made of this in the future.

\subsubsection{Finding Relations}

Our starting assumption is that any such relations we use should be valid for particles of any size. As a result, in order to identify relations, we can vary the size of the particle (by varying $x$ here), carry out the integrations for each size, and test the linear dependence of the resulting integrals.

By means of illustration, if we have equations

$$
\begin{aligned}
& f_{1}(x)=x \\
& f_{2}(x)=3 \\
& f_{3}(x)=3 x+6
\end{aligned}
$$

then we can simply see that $3 f_{1}(x)+2 f_{2}(x)=f_{3}(x)$. 
In order to find this relation numerically, we would evaluate the equations for several different values of $x$, and form a matrix with the results. In this case, as there are three equations, we only need to evaluate at three points. This leads to a matrix like

$$
\begin{aligned}
& f_{1}(x) \\
& x=1 \\
& x=2 \\
& x=3
\end{aligned} \quad\left(\begin{array}{ccc}
1 & f_{2}(x) & f_{3}(x) \\
2 & 3 & 9 \\
3 & 3 & 12
\end{array}\right)
$$

and the rank of this matrix is two. Thus, we may deduce that there is a linear relation, though it is not necessarily obvious from knowing the rank what the relations are. Of course, if there were two independent linear relations between the elements, then the rank would be one.

In our context, we have a matrix where each column represents a different matrix element, and each row represents a different $x$-value. Unfortunately, it is numerically challenging to determine the rank of a large matrix accurately, and for this task MATLAB under-reports the rank. This is evident because as we reduce the number of columns in our matrix, which cannot increase the rank as we are not adding information, the reported rank increases. However, taking the rank of small subsets of the matrix results in a rank-deficient matrix, and by testing the effect of removing columns, we may determine which elements have linear relations between them.

This method is valuable for determining where the relationships are, but it is a challenge to select only the simplest relations. That is, if there is some element $a$ expressable as a combination

$$
a=f(b, c, d)
$$

then another element $e$, expressable as

$$
e=f^{\prime}(a, f, g, h)
$$


will also be expressable as

$$
e=f^{\prime \prime}(b, c, d, f, g, h)
$$

Thus, we only wish to find relationships that do not contain simpler relationships.

\subsubsection{Obtaining Exact Relations}

The approach in the previous section is useful to determine that there are relations, but it is not well suited to finding exact expressions. Instead, we look at the expressions, and attempt to find relations between them, using the results of the previous section as a guide as to which elements to focus on. Some of the relations we already have, such as the relation between $\mathbf{L}^{1}, \mathbf{L}^{2}, \mathbf{L}^{3}$ and $\mathbf{L}^{4}$ (where only three of those are linearly independent), as shown in Eq. (4.44). Also trivially, on the diagonal, $L_{n n}^{1}=L_{n n}^{2}$. Finding other relations is less easy.

We start by defining slightly different forms of the integrals, replacing our angular functions with the associated Legendre functions, as we are now focused on simplicity of the expressions and not numerical stability in calculating them. We make use of

$$
\begin{aligned}
P_{n} & =C_{n} d_{n}=P_{n}^{m}(\cos \theta) \\
T_{n} & =C_{n} \tau_{n}=\frac{\mathrm{d}}{\mathrm{d} \theta} P_{n}^{m}(\cos \theta) \\
C_{n} & =(-1)^{m} \sqrt{\frac{(n+m) !}{(n-m) !}}
\end{aligned}
$$

where the index $m$ and parameter $\theta$ are implicit, and $d_{n} \equiv d_{n m}(\theta)$ and $\tau_{n} \equiv \tau_{n m}(\theta)$. Thus, for reasons of convenience, we work with the functions

$$
\begin{gathered}
\tilde{K}^{1}=C_{n} C_{k} m d_{n} d_{k} x_{\theta} \chi_{n} \psi_{k}^{\prime}=m P_{n} P_{k} x_{\theta} \chi_{n} \psi_{k}^{\prime} \\
\tilde{K}^{2}=C_{n} C_{k} m d_{n} d_{k} x_{\theta} \chi_{n}^{\prime} \psi_{k}=m P_{n} P_{k} x_{\theta} \chi_{n}^{\prime} \psi_{k} \\
\tilde{L}^{1}=\sin \theta C_{n} C_{k} \tau_{n} d_{k} x_{\theta} \chi_{n} \psi_{k}=\sin \theta T_{n} P_{k} x_{\theta} \chi_{n} \psi_{k} \\
\tilde{L}^{2}=\sin \theta C_{n} C_{k} d_{n} \tau_{k} x_{\theta} \chi_{n} \psi_{k}=\sin \theta P_{n} T_{k} x_{\theta} \chi_{n} \psi_{k}
\end{gathered}
$$


where $\tilde{K}^{1}$ is the integrand of $\mathbf{K}^{1}$, excluding the prefactor $a_{n} a_{k}$, and likewise for the other expressions. Note here that $\tilde{L}_{n k}^{1}$ and $\tilde{L}_{n k}^{2}$ are different to the quantities $\tilde{L}_{n}^{1}$ and $\tilde{L}_{n}^{2}$ that were introduced in Section 4.1.4, which are vectors and not matrices. As we shall see, there exist relations on the integrand, and we do not need to work with the integral.

\section{Angular Relations}

We also make use of the following relations, which are derived and presented in Appendix B, but are reproduced here for convenience;

$$
\begin{aligned}
\pi_{n} \sin \theta & =m d_{n} \\
d_{n}^{\prime} & =\tau_{n} \\
{\left[\pi_{n} \tau_{k}+\tau_{n} \pi_{k}\right] \sin \theta } & =m\left(d_{n} d_{k}\right)^{\prime} \\
{\left[\pi_{n} \pi_{k}+\tau_{n} \tau_{k}\right] \sin \theta } & =\left(d_{n} \tau_{k} \sin \theta\right)^{\prime}+k(k+1) d_{n} d_{k} \sin \theta \\
{\left[\pi_{n} \pi_{k}+\tau_{n} \tau_{k}\right] \sin \theta } & =\left(\tau_{n} d_{k} \sin \theta\right)^{\prime}+n(n+1) d_{n} d_{k} \sin \theta \\
\left(\tau_{n} d_{k} \sin \theta\right)^{\prime} & =\sin \theta\left(\tau_{n} \tau_{k}+\pi_{n} \pi_{k}-n(n+1) d_{n} d_{k}\right) \\
\left(d_{n} \tau_{k} \sin \theta\right)^{\prime} & =\sin \theta\left(\tau_{n} \tau_{k}+\pi_{n} \pi_{k}-k(k+1) d_{n} d_{k}\right) \\
\left(d_{n} \sin \theta\right)^{\prime} & =\tau_{n} \sin \theta+d_{n} \cos \theta \\
\left(\tau_{n} \sin \theta\right)^{\prime} & =m \pi_{n}-n(n+1) d_{n} \sin \theta \\
\left(\pi_{n} \sin \theta\right)^{\prime} & =m \tau_{n} .
\end{aligned}
$$

We may also use the recursion relations for the angular functions to obtain the additional relations

$$
\begin{aligned}
(n-m+1) P_{n+1}+(n+1) P_{n-1} & =(2 n+1) \cos \theta P_{n} \\
n \cos \theta P_{n}-\sin \theta T_{n} & =(n+1) P_{n-1} \\
(n+1) \cos \theta P_{n}+\sin \theta T_{n} & =(n-m+1) P_{n+1} \\
n(n-m+1) P_{n+1}-(n+1)(n+m) P_{n-1} & =(2 n+1) \sin \theta T_{n}
\end{aligned}
$$


We here consider only the case where $m=1$, and other cases would behave similarly, though this work has not been carried out. Note that in this case, $C_{n}$ reduces to

$$
C_{n}=\sqrt{n(n+1)}
$$

From Eq. 4.83 we may obtain

$$
(2 k+1)\left[n P_{n+1} P_{k}+(n+1) P_{n-1} P_{k}\right]=(2 n+1)\left[k P_{n} P_{k}+(n+1) P_{n-1} P_{k}\right]
$$

and from Eq. (4.86) we have

$$
(2 k+1)\left[n^{2} P_{n+1} T_{k}-(n+1)^{2} P_{n-1} T_{k}\right]=(2 n+1)\left[k^{2} T_{n} P_{k+1}-(k+1)^{2} T_{n} P_{k-1}\right]
$$

It is also possible to obtain from Eqs. (4.84) and 4.85 the set of four equations, three of which are linearly independent

$$
\begin{aligned}
\sin \theta\left[k T_{n} P_{k}-n P_{n} T_{k}\right] & =n(k+1) P_{n} P_{k-1}-k(n+1) P_{n-1} P_{k} \\
\sin \theta\left[(k+1) T_{n} P_{k}+n P_{n} T_{k}\right] & =n k P_{n} P_{k+1}-(k+1)(n+1) P_{n-1} P_{k} \\
\sin \theta\left[k T_{n} P_{k}+(n+1) P_{n} T_{k}\right] & =n k P_{n+1} P_{k}-(k+1)(n+1) P_{n} P_{k-1} \\
\sin \theta\left[(k+1) T_{n} P_{k}-(n+1) P_{n} T_{k}\right] & =n(k+1) P_{n+1} P_{k}-k(n+1) P_{n} P_{k+1}
\end{aligned}
$$

\section{Radial Relations}

There are also some relations on the radial functions that are useful. The starting point for these is the differential equation which defines the Riccati-Bessel functions, Eq. 4.23), reproduced here for convenience

$$
w_{n}^{\prime \prime}(x)=\left[\frac{n(n+1)}{x^{2}}-1\right] w_{n}(x)
$$


where here $w(x)$ can be any of the Riccati-Bessel functions. From this, we may obtain

$$
\begin{aligned}
\frac{\mathrm{d}}{\mathrm{d} \theta}\left[k(k+1) \chi_{n}^{\prime} \psi_{k}-n(n+1) s \chi_{n} \psi_{k}^{\prime}\right]= & {\left[n(n+1) s^{2}-k(k+1)\right] x_{\theta} \chi_{n} \psi_{k} } \\
& -[n(n+1)-k(k+1)] s x_{\theta} \chi_{n}^{\prime} \psi_{k}^{\prime}
\end{aligned}
$$

There are recursion relations on these functions, which are

$$
\begin{aligned}
w_{n-1}(x)+w_{n+1}(x) & =\frac{2 n+1}{x} w_{n}(x) \\
\frac{n}{x} w_{n}(x)+w_{n}^{\prime}(x) & =w_{n-1}(x) \\
\frac{n+1}{x} w_{n}(x)-w_{n}^{\prime}(x) & =w_{n+1}(x) \\
(n+1) w_{n-1}(x)-n w_{n+1}(x) & =(2 n+1) w_{n}^{\prime}(x) .
\end{aligned}
$$

It is possible to combine these to obtain relations, noting that the argument of the regular Bessel function $\psi$ is here $s x$,

$$
\begin{aligned}
k \chi_{n}^{\prime} \psi_{k}-n s \chi_{n} \psi_{k}^{\prime} & =k \chi_{n-1} \psi_{k}-n s \chi_{n} \psi_{k-1} \\
(k+1) \chi_{n}^{\prime} \psi_{k}+n s \chi_{n} \psi_{k}^{\prime} & =(k+1) \chi_{n-1} \psi_{k}-n s \chi_{n} \psi_{k+1} \\
k \chi_{n}^{\prime} \psi_{k}+(n+1) s \chi_{n} \psi_{k}^{\prime} & =(n+1) s \chi_{n} \psi_{k-1}-k \chi_{n+1} \psi_{k} \\
(k+1) \chi_{n}^{\prime} \psi_{k}-(n+1) s \chi_{n} \psi_{k}^{\prime} & =(n+1) s \chi_{n} \psi_{k+1}-(k+1) \chi_{n+1} \psi_{k} .
\end{aligned}
$$

We start by looking at some special cases in the following sections. 


\section{Special Case 1}

We look for a relation between the elements $\tilde{L}_{31}^{1}, \tilde{L}_{31}^{2}, \tilde{L}_{11}^{1}$ and $\tilde{K}_{21}^{2}$. Thus, the relation is between

$$
\begin{array}{ll}
\chi_{3} \psi_{1}, & T_{3} P_{1} \sin \theta \\
\chi_{3} \psi_{1}, & T_{1} D_{3} \sin \theta \\
\chi_{1} \psi_{1}, & T_{1} P_{1} \sin \theta \\
\chi_{2}^{\prime} \psi_{1}, & P_{2} P_{1}
\end{array}
$$

(that is, radial terms from the first column and angular terms from the second column are paired together). This special case is near the diagonal, and also on the edge of the matrix, so we expect that the relation could be simpler than a general relation. Checking the rank of the appropriately-constructed matrix, it is only 3 , indicating that the four entries are not linearly independent.

By using $n=1$ in Eq. 4.86, and noting that $P_{0}=0$, we have

$$
P_{2}=3 \sin \theta T_{1}
$$

Also setting $n=3, k=1$ in Eq. (4.90) we have

$$
\sin \theta\left[T_{3} P_{1}-3 P_{3} T_{1}\right]=-4 P_{2} P_{1}
$$

and by using $n=2$ in Eq. 4.99) (with $w=\chi$ ), and by multiplying by $\psi_{1}$ we have

$$
2 \chi_{3} \psi_{1}=3 \chi_{1} \psi_{1}-5 \chi_{2}^{\prime} \psi_{1}
$$

Combining all of these we have

$$
\begin{aligned}
\sin \theta\left[T_{3} P_{1} \chi_{3} \psi_{1}-3 P_{3} T_{1} \chi_{3} \psi_{1}\right] & =2\left[5 P_{2} P_{1} \chi_{2}^{\prime} \psi_{1}-3 P_{2} P_{1} \chi_{1} \psi_{1}\right] \\
& =2\left[5 P_{2} P_{1} \chi_{2}^{\prime} \psi_{1}-9 \sin \theta P_{1} T_{1} \chi_{1} \psi_{1}\right] \\
\tilde{L}_{31}^{1}-3 \tilde{L}_{31}^{2} & =10 \tilde{K}_{21}^{2}-18 \tilde{L}_{11}^{1}
\end{aligned}
$$


We may also use the same relations to obtain the symmetric case,

$$
\tilde{L}_{13}^{2}-3 \tilde{L}_{13}^{1}=10 \tilde{K}_{12}^{1}-6 \tilde{L}_{11}^{1}
$$

Thus we see that is is possible to obtain some relations on elements of $\mathbf{U}$. Note that the relations are valid on $\mathbf{P}$ also, as all the relations on $\chi_{n} \psi_{k}$ are also valid on $\psi_{n} \psi_{k}$.

\section{Special Case 2}

While it is possible to find other relations between four entries, as in Special Case 1, it is worth exploring relations between more elements. Here we consider a relation between $\tilde{L}_{n, 2}^{1}, \tilde{L}_{n, 2}^{2}, \tilde{L}_{n-1,1}^{1}, \tilde{L}_{n-1,1}^{2}, \tilde{K}_{n-1,2}^{1}, \tilde{K}_{n-1,2}^{2}, \tilde{K}_{n, 1}^{1}$ and $\tilde{K}_{n, 1}^{2}$. By means of illustration, this is between the following entries shown in Fig. 4.1 The rank of the appropriate matrix is only seven, indicating that there is a linear relation between these eight elements.

$$
\begin{aligned}
& \mathbf{L}^{1}, \mathbf{L}^{2} \quad \mathbf{K}^{1}, \mathbf{K}^{2}
\end{aligned}
$$

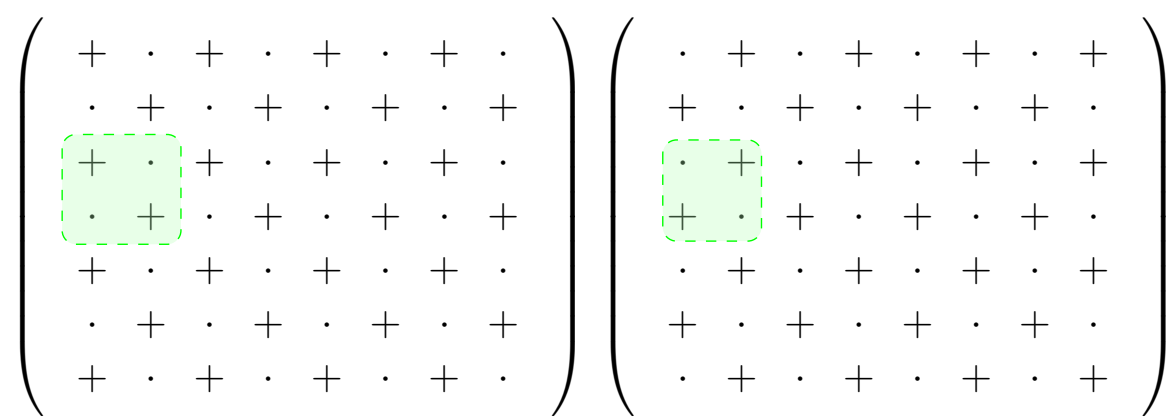

Figure 4.1: A depiction of which entries are related by Special Case 2, where here the left matrix represents both $\mathbf{L}^{1}$ and $\mathbf{L}^{2}$, and the right matrix both $\mathbf{K}^{1}$ and $\mathbf{K}^{2}$. Here entries which have the same parity of $n+k$ as our relation are shown as $\cdot$, while off-parity entries are shown as + . This is shown here for $n=4$, but the relation is valid for other values of $n$ also.

We start with Eq. 4.90, and set $n=n, k=2$. This gives

$$
\sin \theta\left[2 T_{n} P_{2}-n P_{n} T_{2}\right]=-2(n+1) P_{n-1} P_{2}+3 n P_{n} P_{1}
$$


This corresponds to the angular parts of $\tilde{L}_{n, 2}^{1}$ and $\tilde{L}_{n, 2}^{2}$, and we may write

$$
\left[2 \tilde{L}_{n, 2}^{1}-n \tilde{L}_{n, 2}^{2}\right]=-2(n+1) \xi_{n} \psi_{n} P_{n-1} P_{2}+3 n \xi_{n} \psi_{2} P_{n} P_{1}
$$

We also may use $n=n-1, k=2$ in Eq. 4.102 to get

$$
-2 \xi_{n} \psi_{2}=2 \xi_{n-1}^{\prime} \psi_{2}+n s \xi_{n-1} \psi_{2}^{\prime}-n s \xi_{n-1} \psi_{1}
$$

and substituting this in we obtain

$$
\left[2 \tilde{L}_{n, 2}^{1}-n \tilde{L}_{n, 2}^{2}\right]=(n+1)\left(-n s \xi_{n-1} \psi_{1} P_{n-1} P_{2}+2 \tilde{K}_{n-1,2}^{2}+n s \tilde{K}_{n-1,2}^{1}\right)+3 n \xi_{n} \psi_{2} P_{n} P_{1}
$$

Using Eq. (4.86), we have $P_{2}=3 \sin \theta T_{1}$, and hence

$$
n s \xi_{n-1} \psi_{1} P_{n-1} P_{2}=3 n s \xi_{n-1} \psi_{1} P_{n-1} T_{1} \sin \theta=3 n s \tilde{L}_{n-1,1}^{2}
$$

For the remaining term, we may use Eq. 4.101 with $n=n, k=1$ to get

$$
n s \xi_{n} \psi_{2}=2 \xi_{n-1} \psi_{1}-2 \xi_{n}^{\prime} \psi_{1}-n s \xi_{n} \psi_{1}^{\prime}
$$

as well as $n=n-1, k=1$ in Eq. 4.92

$$
\sin \theta\left[T_{n-1} P_{1}+n P_{n-1} T_{1}\right]=(n-1) P_{n} P_{1}
$$

and hence

$$
\begin{aligned}
3 n \xi_{n} \psi_{2} P_{n} P_{1} & =\frac{6}{s} \xi_{n-1} \psi_{1} P_{n} P_{1}-\frac{6}{s} \xi_{n}^{\prime} \psi_{1} P_{n} P_{1}-3 n \xi_{n} \psi_{1}^{\prime} P_{n} P_{1} \\
& =\frac{6}{s} \xi_{n-1} \psi_{1} P_{n} P_{1}-3\left[\frac{2}{s} \tilde{K}_{n, 1}^{2}-n \tilde{K}_{n, 1}^{1}\right] \\
& =\frac{6 \sin \theta}{s(n-1)} \xi_{n-1} \psi_{1}\left[T_{n-1} P_{1}+n P_{n-1} T_{1}\right]-3\left[\frac{2}{s} \tilde{K}_{n, 1}^{2}-n \tilde{K}_{n, 1}^{1}\right] \\
& =\frac{6}{s(n-1)}\left[\tilde{L}_{n-1,1}^{1}+n \tilde{L}_{n-1,1}^{2}\right]-3\left[\frac{2}{s} \tilde{K}_{n, 1}^{2}-n \tilde{K}_{n, 1}^{1}\right] .
\end{aligned}
$$


Thus, we may combine all of these results to obtain

$$
\begin{aligned}
2 \tilde{L}_{n, 2}^{1}-n \tilde{L}_{n, 2}^{2}= & (n+1)\left[2 \tilde{K}_{n-1,2}^{2}+n s \tilde{K}_{n-1,2}^{1}\right]-3\left[\frac{2}{s} \tilde{K}_{n, 1}^{2}-n \tilde{K}_{n, 1}^{1}\right] \\
& +\frac{6}{s(n-1)}\left[\tilde{L}_{n-1,1}^{1}+n\left(1-\frac{s^{2}\left(n^{2}-1\right)}{2}\right) \tilde{L}_{n-1,1}^{2}\right]
\end{aligned}
$$

This relation is valid on the edge of the matrix. Symmetric cases (for $n=2$, $k=k$ ) may be easily derived by symmetry.

\section{General Case 1}

We now move on to a more general case, which is not necessarily near the diagonal or the edge of the matrix. The elements that this relation is between are depicted in Fig. 4.2. The elements involved in this relation are $\tilde{L}_{n, k+1}^{1}, \tilde{L}_{n, k+1}^{2}, \tilde{L}_{n, k-1}^{1}, \tilde{L}_{n, k-1}^{2}$, $\tilde{K}_{n, k}^{1}, \tilde{K}_{n, k}^{2}, \tilde{K}_{n-1, k+1}^{1}, \tilde{K}_{n-1, k+1}^{2}, \tilde{K}_{n-1, k-1}^{1}, \tilde{K}_{n-1, k-1}^{2}, \tilde{L}_{n-1, k}^{1}$ and $\tilde{L}_{n-1, k}^{2}$.

$$
\left(\begin{array}{cccccccc}
\multicolumn{1}{c}{\mathbf{L}^{1}, \mathbf{L}^{2}} & & & \mathbf{K}^{1}, \mathbf{K}^{2} & & \\
+ & \cdot & + & \cdot & + & \cdot & + & \cdot \\
\cdot & + & \cdot & + & \cdot & + & \cdot & + \\
+ & \cdot & + & \cdot & + & \cdot & + & \cdot \\
\cdot & + & \cdot & + & \cdot & + & \cdot & + \\
+ & \cdot & + & \cdot & + & \cdot & + & \cdot \\
\cdot & + & \cdot & + & \cdot & + & \cdot & + \\
+ & \cdot & + & \cdot & + & \cdot & + & \cdot
\end{array}\right)\left(\begin{array}{cccccccc}
\cdot & + & \cdot & + & \cdot & + & \cdot & + \\
+ & \cdot & + & \cdot & + & \cdot & + & \cdot \\
\cdot & + & \cdot & + & \cdot & + & \cdot & + \\
+ & \cdot & + & \cdot & + & \cdot & + & \cdot \\
\cdot & + & \cdot & + & \cdot & + & \cdot & + \\
+ & \cdot & + & \cdot & + & \cdot & + & \cdot \\
\hdashline & + & \cdot & + & \cdot & + & \cdot & +
\end{array}\right)
$$

Figure 4.2: A depiction of which entries are related by General Case 1, where here the left matrix represents both $\mathbf{L}^{1}$ and $\mathbf{L}^{2}$, and the right matrix both $\mathbf{K}^{1}$ and $\mathbf{K}^{2}$. Here entries which are used in this relation are shown as + , while non-contributing entries are shown as $\cdot$. This is shown here for $n=6$ and $k=3$, but the relation is valid for other values of $n$ and $k$.

We start by writing Eq. 4.90 with $n=n, k=k+1$, and multiplied by $\chi_{n} \psi_{k+1}$, 
which is

$$
(k+1) \tilde{L}_{n, k+1}^{1}-n \tilde{L}_{n, k+1}^{2}=\chi_{n} \psi_{k+1}\left(n(k+2) P_{n} P_{k}-(k+1)(n+1) P_{n-1} P_{k+1}\right) .
$$

We then make use of Eq. (4.102, with $n=n-1$ and $k=k+1$, which is

$$
-\chi_{n} \psi_{k+1}(k+1)=(k+1) \chi_{n-1}^{\prime} \psi_{k+1}+n s \chi_{n-1} \psi_{k+1}^{\prime}-n s \chi_{n-1} \psi_{k}
$$

and Eq. 4.101) with $n=n, k=k$, which is

$$
n \chi_{n} \psi_{k+1}=\frac{k+1}{s} \chi_{n-1} \psi_{k}-\frac{k+1}{s} \chi_{n}^{\prime} \psi_{k}-n \chi_{n} \psi_{k}^{\prime}
$$

Combining all of these, we are left with

$$
\begin{aligned}
(k+1) \tilde{L}_{n, k+1}^{1}-n \tilde{L}_{n, k+1}^{2}= & (k+2) P_{n} P_{k}\left(\frac{k+1}{s} \chi_{n-1} \psi_{k}-\frac{k+1}{s} \chi_{n}^{\prime} \psi_{k}-n \chi_{n} \psi_{k}^{\prime}\right) \\
& +(n+1) P_{n-1} P_{k+1}\left((k+1) \chi_{n-1}^{\prime} \psi_{k+1}+n s \chi_{n-1} \psi_{k+1}^{\prime}-n s \chi_{n-1} \psi_{k}\right)
\end{aligned}
$$

in which we may recognise some integral forms, leaving

$$
\begin{aligned}
(k+1) \tilde{L}_{n, k+1}^{1}-n \tilde{L}_{n, k+1}^{2}= & -\frac{(k+1)(k+2)}{s} \tilde{K}_{n k}^{2}-n(k+2) \tilde{K}_{n k}^{1}+ \\
& +(n+1)(k+1) \tilde{K}_{n-1, k+1}^{2}+n(n+1) s \tilde{K}_{n-1, k+1}^{1}+A
\end{aligned}
$$

where we group the parts that do not yet resemble integrals into $A$ such that

$$
A=\frac{(k+1)(k+2)}{s} P_{n} P_{k} \chi_{n-1} \psi_{k}-n(n+1) s P_{n-1} P_{k+1} \chi_{n-1} \psi_{k}
$$

Setting $A$ aside for now, we now make use of Eq. (4.91) to move in the other 
direction, using $n=n-1, k=k-1$. This is

$$
k \tilde{L}_{n, k-1}^{1}+n \tilde{L}_{n, k-1}^{2}=\chi_{n} \psi_{k-1}\left(n(k-1) P_{n} P_{k}-k(n+1) P_{n-1} P_{k-1}\right)
$$

and then by using Eq. 4.103 with $n=n-1, k=k-1$, we have

$$
-k \chi_{n} \psi_{k-1}=k \chi_{n-1}^{\prime} \psi_{k-1}-n s \chi_{n-1} \psi_{k-1}^{\prime}-n s \chi_{n-1} \psi_{k}
$$

and by using Eq. (4.100) with $n=n, k=k$ we have

$$
n \chi_{n} \psi_{k-1}=\frac{k}{s} \chi_{n-1} \psi_{k}+(n+1) \chi_{n} \psi_{k}^{\prime}-\frac{k}{s} \chi_{n}^{\prime} \psi_{k}
$$

These may all be combined to yield

$$
\begin{aligned}
k \tilde{L}_{n, k-1}^{1}+n \tilde{L}_{n, k-1}^{2}= & (k-1) P_{n} P_{k}\left(\frac{k}{s} \chi_{n-1} \psi_{k}+(n+1) \chi_{n} \psi_{k}^{\prime}-\frac{k}{s} \chi_{n}^{\prime} \psi_{k}\right) \\
& +(n+1) P_{n-1} P_{k-1}\left(k \chi_{n-1}^{\prime} \psi_{k-1}-n s \chi_{n-1} \psi_{k-1}^{\prime}-n s \chi_{n-1} \psi_{k}\right)
\end{aligned}
$$

and again recognising some integral forms,

$$
\begin{aligned}
k \tilde{L}_{n, k-1}^{1}+n \tilde{L}_{n, k-1}^{2}= & (n+1)(k-1) \tilde{K}_{n k}^{1}-\frac{k(k-1)}{s} \tilde{K}_{n k}^{2} \\
& +k(n+1) \tilde{K}_{n-1, k-1}^{2}-n(n+1) s \tilde{K}_{n-1, k-1}^{1}+B
\end{aligned}
$$

where we have grouped terms to create $B$ as

$$
B=\frac{k(k-1)}{s} P_{n} P_{k} \chi_{n-1} \psi_{k}-n(n+1) s P_{n-1} P_{k-1} \chi_{n-1} \psi_{k}
$$

It is now a matter of working with $A$ and $B$ to recast them in terms of integrals that we know, now by working with the angular functions. Firstly, we use Eq. 4.92 
with $n=n-1, k=k$ to write

$$
\begin{aligned}
B & =\left(\frac{k(k-1)}{s}-\frac{k\left(n^{2}-1\right) s}{k+1}\right) P_{n} P_{k} \chi_{n-1} \psi_{k}+\frac{s(n+1)}{k+1}\left(k \tilde{L}_{n-1, k}^{1}-n \tilde{L}_{n-1, k}^{2}\right) \\
B^{\prime} & =\left(\frac{k(k-1)}{s}-\frac{k\left(n^{2}-1\right) s}{k+1}\right) P_{n} P_{k} \chi_{n-1} \psi_{k}
\end{aligned}
$$

and secondly we use Eq. 4.93 with $n=n-1, k=k$ to get

$$
\begin{aligned}
& A=(k+1) P_{n} P_{k} \chi_{n-1} \psi_{k}\left(\frac{(k+2)}{s}-\frac{\left(n^{2}-1\right) s}{k}\right)+\frac{s(n+1)}{k}\left((k+1) \tilde{L}_{n-1, k}^{1}-n \tilde{L}_{n-1, k}^{2}\right) \\
& A^{\prime}=(k+1) P_{n} P_{k} \chi_{n-1} \psi_{k}\left(\frac{(k+2)}{s}-\frac{\left(n^{2}-1\right) s}{k}\right)
\end{aligned}
$$

Now we note that the non-integral terms in $A^{\prime}$ and $B^{\prime}$ have the same angular and radial functions, and hence the appropriate combination of $(k+1) \tilde{L}_{n, k+1}^{1}-n \tilde{L}_{n, k+1}^{2}$ and $k \tilde{L}_{n, k-1}^{1}+n \tilde{L}_{n, k-1}^{2}$ should produce a relation with only integral forms in it. This 
may be expressed as

$$
\begin{aligned}
\alpha\left((k+1) \tilde{L}_{n, k+1}^{1}-n \tilde{L}_{n, k+1}^{2}\right) & \\
-\beta\left(k \tilde{L}_{n, k-1}^{1}+n \tilde{L}_{n, k-1}^{2}\right)= & \\
& -\alpha \frac{(k+1)(k+2)}{s} \tilde{K}_{n k}^{2} \\
& -\alpha n(k+2) \tilde{K}_{n k}^{1} \\
& +\alpha(n+1)(k+1) \tilde{K}_{n-1, k+1}^{2} \\
& +\alpha n(n+1) s \tilde{K}_{n-1, k+1}^{1} \\
& +\alpha \frac{s(n+1)}{k}(k+1) \tilde{L}_{n-1, k}^{1} \\
& -\alpha \frac{n(n+1) s}{k} \tilde{L}_{n-1, k}^{2} \\
& +\alpha A^{\prime} \\
& -\beta(n+1)(k-1) \tilde{K}_{n k}^{1} \\
& +\beta \frac{k(k-1)}{s} \tilde{K}_{n k}^{2} \\
& -\beta k(n+1) \tilde{K}_{n-1, k-1}^{2} \\
& +\beta n(n+1) s \tilde{K}_{n-1, k-1}^{1} \\
& -\beta \frac{s(n+1)}{k+1} k \tilde{L}_{n-1, k}^{1} \\
& +\beta \frac{n(n+1) s}{k+1} \tilde{L}_{n-1, k}^{2} \\
& -\beta B^{\prime}
\end{aligned}
$$

for some values $\alpha$ and $\beta$, where we clearly require here that $\alpha A^{\prime}=\beta B^{\prime}$, which means that

$\alpha(k+1) P_{n} P_{k} \chi_{n-1} \psi_{k}\left(\frac{(k+2)}{s}-\frac{\left(n^{2}-1\right) s}{k}\right)=\beta\left(\frac{k(k-1)}{s}-\frac{k\left(n^{2}-1\right) s}{k+1}\right) P_{n} P_{k} \chi_{n-1} \psi_{k}$ 
and so we may express the ratio $\alpha / \beta$ as

$$
\frac{\alpha}{\beta}=k^{2} \frac{\left(k^{2}-1\right)-s^{2}\left(n^{2}-1\right)}{(k+1)^{2}\left(k(k+2)-\left(n^{2}-1\right) s^{2}\right)} .
$$

With this choice of $\alpha / \beta$, we may then write our relation as

$$
\begin{aligned}
\alpha\left((k+1) \tilde{L}_{n, k+1}^{1}-n \tilde{L}_{n, k+1}^{2}\right) & \\
-\beta\left(k \tilde{L}_{n, k-1}^{1}+n \tilde{L}_{n, k-1}^{2}\right)= & \\
& -(\alpha n(k+2)+\beta(n+1)(k-1)) \tilde{K}_{n k}^{1} \\
& +\left(\beta \frac{k(k-1)}{s}-\alpha \frac{(k+1)(k+2)}{s}\right) \tilde{K}_{n k}^{2} \\
& +\alpha(n+1)\left((k+1) \tilde{K}_{n-1, k+1}^{2}+n s \tilde{K}_{n-1, k+1}^{1}\right) \\
& -\beta(n+1)\left(k \tilde{K}_{n-1, k-1}^{2}-n s \tilde{K}_{n-1, k-1}^{1}\right) \\
& +\left(\alpha \frac{s(n+1)(k+1)}{k}-\beta \frac{k(n+1) s}{k+1}\right) \tilde{L}_{n-1, k}^{1} \\
& +\left(\beta \frac{n(n+1) s}{k+1}-\alpha \frac{n(n+1) s}{k}\right) \tilde{L}_{n-1, k}^{2} .
\end{aligned}
$$

\section{General Case 2}

Following on from the General Case 1 in the previous section, it is possible to find a relation between another 12 elements, as shown in Fig. 4.3. Luckily, the analytic relation here may be found easily from the form of General Case 1, by simply swapping $n$ and $k$ in all expressions, and we do not need to repeat the entire derivation. Note that this results in exchanging $\mathbf{L}^{1}$ and $\mathbf{L}^{2}$, as well as $\mathbf{K}^{1}$ and $\mathbf{K}^{2}$. 
The expression is then

$$
\begin{aligned}
\gamma\left((n+1) \tilde{L}_{n+1, k}^{2}-k \tilde{L}_{n+1, k}^{1}\right) & \\
-\delta\left(n \tilde{L}_{n-1, k}^{2}+k \tilde{L}_{n-1, k}^{1}\right)= & -(\gamma k(n+2)+\delta(k+1)(n-1)) \tilde{K}_{n k}^{2} \\
& +\left(\delta \frac{n(n-1)}{s}-\gamma \frac{(n+1)(n+2)}{s}\right) \tilde{K}_{n k}^{1} \\
& +\gamma(k+1)\left((n+1) \tilde{K}_{n+1, k-1}^{1}+k s \tilde{K}_{n+1, k-1}^{2}\right) \\
& -\delta(k+1)\left(n \tilde{K}_{n-1, k-1}^{1}-k s \tilde{K}_{n-1, k-1}^{2}\right) \\
& +\left(\gamma \frac{s(k+1)(n+1)}{n}-\delta \frac{n(k+1) s}{n+1}\right) \tilde{L}_{n, k-1}^{2} \\
& +\left(\delta \frac{k(k+1) s}{n+1}-\gamma \frac{k(k+1) s}{n}\right) \tilde{L}_{n, k-1}^{1} .
\end{aligned}
$$

where the expressions for $\gamma$ and $\delta$ are simply obtained by exchanging $n$ and $k$ in the expressions for $\alpha$ and $\beta$ respectively, giving the ratio

$$
\frac{\gamma}{\delta}=n^{2} \frac{\left(n^{2}-1\right)-s^{2}\left(k^{2}-1\right)}{(n+1)^{2}\left(n(n+2)-\left(k^{2}-1\right) s^{2}\right)} .
$$

\subsubsection{Using the Relations}

Now that we have some relations between elements, we may naturally ask some questions. Firstly, have we found all of the relations? Secondly, given the relations we have, is it possible to make use of them in order to calculate the $T$-matrix more efficiently?

Regarding the first question, we can answer "yes" under certain conditions. If we consider only the $m=1$ case, and only the matrices $\mathbf{L}^{1}, \mathbf{L}^{2}, \mathbf{K}^{1}$ and $\mathbf{K}^{2}$ (that is, excluding $\mathbf{L}^{3}$ and $\mathbf{L}^{4}$ ), then these relations serve to fully explain the reduced rank. Of course, we may construct other relations as combinations of these two, or special cases, such as near the edge or diagonal, but these relations form a basis for the 


$$
\begin{aligned}
& \mathbf{L}^{1}, \mathbf{L}^{2} \quad \mathbf{K}^{1}, \mathbf{K}^{2} \\
& \left(\begin{array}{cccccccc}
+ & \cdot & + & \cdot & + & \cdot & + & \cdot \\
\cdot & + & \cdot & + & \cdot & + & \cdot & + \\
+ & \cdot & + & \cdot & + & \cdot & + & \cdot \\
\cdot & + & \cdot & + & \cdot & + & \cdot & + \\
+ & \cdot & + & \cdot & + & \cdot & + & \cdot \\
\cdot & + & \cdot & + & \cdot & + & \cdot & + \\
+ & \cdot & + & \cdot & + & \cdot & + & \cdot
\end{array}\right)\left(\begin{array}{cccccccc}
\cdot & + & \cdot & + & \cdot & + & \cdot & + \\
+ & \cdot & + & \cdot & + & \cdot & + & \cdot \\
\cdot & + & \cdot & + & \cdot & + & \cdot & + \\
+ & \cdot & + & \cdot & + & \cdot & + & \cdot \\
\cdot & + & \cdot & + & \cdot & + & \cdot & + \\
+ & \cdot & + & \cdot & + & \cdot & + & \cdot \\
\cdot & + & \cdot & + & \cdot & + & \cdot & +
\end{array}\right)
\end{aligned}
$$

Figure 4.3: A depiction of which entries are related by General Case 2, where here the left matrix represents both $\mathbf{L}^{1}$ and $\mathbf{L}^{2}$, and the right matrix both $\mathbf{K}^{1}$ and $\mathbf{K}^{2}$. Here entries which are involved in the relation are shown as + , while entries that are not involved are shown as $\cdot$ This is shown here for $n=6$ and $k=3$, but the relation is valid for other values of $n$ and $k$.

other relations. The other proviso is that these relations are valid on the integrand. There are some relations valid on the integral, which means that they only hold for certain shapes. As a result, we do not consider these, as they are not general. Our relations are valid for all axisymmetric particles.

For the second question, the answer is much easier to state, in that we do not need to qualify it to such a degree. These relations do not make it easier to calculate the $T$-matrix. It is worth, however, considering what we mean by this, and what improvements are necessary to be able to answer "yes" to this question. For a fixed $m$, using this recursion scheme to calculate the $T$-matrix up to $n=N$, we must calculate $\mathcal{O}\left(N^{2}\right)$ elements.1 However, there are $\mathcal{O}\left(N^{2}\right)$ elements, so there is no major gain to be made compared to evaluating every integral. As well as this, even if it was more efficient, there are still the elements in $\mathbf{L}^{3}$ and $\mathbf{L}^{4}$ to be calculated, and as long as we have no relations involving them, then the overall behaviour will not improve.

There is an obvious means that could result in a much more useful result. If

\footnotetext{
${ }^{1}$ In so-called "Big-O" notation, this means that in the limit of large numbers of terms, the number of operations required to calculate the matrix scales as $N^{2}$. Broadly speaking, $\mathcal{O}\left(N^{2}\right)$ algorithms outperform $\mathcal{O}\left(N^{3}\right)$ algorithms (or in fact any power larger than 2) as $N$ increases, though for smaller sizes this may not be true.
} 


\subsection{Summary}

a relation could be found (both its existence confirmed, and an exact form found) relating terms of different $m$, then this may enable more efficient calculation of the $T$-matrix (if coupled with a relation involving $\mathbf{L}^{3}$ and $\left.\mathbf{L}^{4}\right)^{2}$. Such a relation, to be of use, would need to not increase the number of terms required for the relation by more than a factor $N$, in order to obtain terms for all $m$ from $m=0$ to $m=N$. As the number of elements goes as $\mathcal{O}\left(N^{3}\right)$, then if the increase is less than a factor of $N$, we would have a significantly more efficient method. Finding (and proving) these relations is work not yet undertaken, and falls sadly outside the scope of this thesis. However, it is anticipated that a concerted effort on this front would be able to make some headway, using the general methods given here for the case $m=1$.

\subsection{Summary}

The results presented in this chapter, previously published in Ref. [1], while offering some small improvements to the EBCM, do not lead to revolutionary gains in the applicability of the EBCM or T-matrix method. The work on obtaining new expressions for the integrals presents a cleaner way of attempting to find more meaningful improvements (such as the attempt to find linear recursion relations), which has led to some significant improvements as shown in the next chapter.

The linear recursion relations shown in this chapter are an unfinished work, and at this point they do not offer an improved method of calculating the $T$-matrix. However, the work undertaken to date may, in the future, lead to relations being found that involve all $m$, which has the potential to reduce the computational cost significantly.

\footnotetext{
${ }^{2}$ As the matrices are not linearly independent, there is no need to include all 4 of them in such a relation. Including 3 of them is sufficient.
} 



\section{Problematic Spheroids}

The spheroid is a geometry that has seen much use as a model for physical systems, in some cases because the spheroid is a good description of the system being studied, and in other cases because it is a simple shape, that adds an additional geometric degree of freedom to the even simpler sphere. One common technique used to study spheroids is the extended boundary-condition method (EBCM). However, it has been known for some time the EBCM suffers from numerical problems in some cases, especially for large or high aspect ratio particles [64]. There have been some limited reports that for certain particle shapes, there are specific numerical problems in the integrals [48, 72, 73]. We have, in Ref. [3], studied the behaviour of the EBCM for the cases of spheroids and offset spheres, and provided a clear explanation of the behaviour for those shapes. Following this, in Ref. [6], we presented a method of modifying the integral expressions for the case of spheroids to remove the numerical problems.

This chapter is based on work from these two publications [3, 6]. To begin with, there is a brief discussion of precision in the context of numerical calculations. Following this is a demonstration of the problems for spheroids. This begins with a demonstration that there are problems, and is followed by a derivation of the cause

Cupcakes (20) Preheat your oven to $190^{\circ} \mathrm{C}$. In a bowl, beat $150 \mathrm{~g}$ softened butter until smooth, and add 1.5 cup white sugar and beat until light and fluffy. Add 2 eggs and mix well. Sift in 2.5 cup self-raising flour, and add $2 \mathrm{tsp}$ vanilla essence and 1.25 cup milk. Beat until smooth. Spoon mixture into paper baking cups in a cupcake tin, so that the cups are $2 / 3$ full. Bake for $20 \mathrm{~min}$, then cool on a wire rack.

Icing Beat $100 \mathrm{~g}$ softened butter until pale and fluffy, then sift in 1.5 cup icing sugar, and add 2-3 drops vanilla essence and 1-2 Tbsp milk. Mix until light and fluffy. Mix in food colouring to reach desired colour. Spread over cooled cupcakes. 


\section{Problematic Spheroids}

of the problems. To conclude, a solution to these problems is provided, which allows calculating a $T$-matrix for spheroids to a high precision with little effort, allowing the treatment of light scattering for spheroids to be about as tractable as for spheres.

The problem that is encountered with the EBCM, including the case of spheroids, is usually observed as a failure of numerical convergence in the calculations. There have been several attempts to overcome this behaviour, often focusing on improving the working precision of the integration, and the matrix inversion [64]. Typically the improved performance of these approaches was attributed to the improved matrix inversion when carried out in higher precision, but we have demonstrated that the inversion of the system is not the cause of the problems for spheroids [3]. Rather, it is the calculation of the integrals that presents problems. However, it is possible to rewrite the relevant integrals in a way that allows them to be easily calculated [6].

In this chapter, for reasons of analytical simplicity, the matrix $\mathbf{U}$ (as defined in Eq. (3.85), such that $\mathbf{Q}=\mathbf{P}+i \mathbf{U}$ ) will be used when working with the expressions instead of $\mathbf{Q}$. However, as $\mathbf{Q}$ is the desired result, numerical examples (such as in the figures) will make use of $\mathbf{Q}$ and not $\mathbf{U}$.

\subsection{Geometries}

Here are presented the geometries used in this chapter. These are the spheroid and the offset sphere, as well as the cylinder.

\subsubsection{Spheroids}

The spheroid, which is used for much of this chapter, is simply a sphere deformed along one of the axes (which is equivalent to an ellipsoid with two of the axes equal). The spheroid is described by

$$
\begin{aligned}
r(\theta) & =\frac{a c}{\sqrt{a^{2} \cos ^{2} \theta+c^{2} \sin ^{2} \theta}} \\
\frac{\mathrm{d} r}{\mathrm{~d} \theta}=r_{\theta} & =\frac{a^{2}-c^{2}}{a^{2} c^{2}} r(\theta)^{3} \sin \theta \cos \theta
\end{aligned}
$$




\subsection{Geometries}

where here $a$ is the semiaxis along the $x$ - and $y$-axes, and $c$ is the semiaxis length along the $z$-axis, which is the axis of revolution.

There are two classes of spheroids that may be considered. Oblate spheroids resemble smarties, and have $a>c$. Prolate spheroids resemble (to a first approximation) a rugby ball, and have $a<c$. The case where $a=c$ is a sphere. The aspect ratio, defined as the ratio between maximum and minimum distances from the origin, is

$$
h=\frac{r_{\max }}{r_{\min }}=\frac{a}{c}
$$

in the case of oblate spheroids, and for prolate spheroids it is

$$
h=\frac{c}{a} .
$$

Often, spheroids are characterised by their equivalent-volume sphere radius $r_{V}$, or their equivalent-area sphere radius, $r_{A}$. The volume of a spheroid is

$$
V=\frac{4}{3} \pi a^{2} c
$$

and hence the equivalent-volume radius is

$$
r_{V}=\sqrt[3]{a^{2} c}
$$

The surface area of a spheroid is

$$
S= \begin{cases}2 \pi a^{2}\left(1+\frac{1-e^{2}}{e} \tanh ^{-1} e\right) & \text { for oblate spheroids } \\ 2 \pi a^{2}\left(1+\frac{c}{a e} \sin ^{-1} e\right) & \text { for prolate spheroids }\end{cases}
$$

where

$$
e^{2}= \begin{cases}1-\frac{c^{2}}{a^{2}} & \text { for oblate spheroids } \\ 1-\frac{a^{2}}{c^{2}} & \text { for prolate spheroids }\end{cases}
$$




\section{Problematic Spheroids}

From this, it is possible to express the equivalent-area sphere radius as

$$
r_{A}= \begin{cases}a \sqrt{\frac{1}{2}+\frac{1-e^{2}}{2 e} \tanh ^{-1} e} & \text { if oblate } \\ a \sqrt{\frac{1}{2}+\frac{c}{2 a e} \sin ^{-1} e} & \text { if prolate. }\end{cases}
$$

These values $r_{V}$ and $r_{A}$ are provided here for reference, but they are not used in this thesis.

\subsubsection{Offset Sphere}

The offset sphere is a sphere (of radius $a$ ) that is displaced along our $z$-axis, the axis of rotation, by some amount $D$. The amount by which it is displaced is less than the radius, to ensure that the origin remains within the shape.

The relevant formulae for an offset sphere are

$$
\begin{aligned}
r(\theta) & =-D \cos \theta+\sqrt{a^{2}-D^{2} \sin ^{2} \theta} \\
r_{\theta} & =D \sin \theta-\frac{D^{2} \sin \theta \cos \theta}{\sqrt{a^{2}-D^{2} \sin ^{2} \theta}} .
\end{aligned}
$$

The offset sphere has an aspect ratio of

$$
h=\frac{a+D}{a-D},
$$

and the surface area and volume are trivially the same as for a sphere of radius $a$. 


\subsection{Numerical Precision}

\subsubsection{Cylinder}

Here we consider a right circular cylinder, with semiaxis a perpendicular to the $z$-axis, and $c$ parallel to $z$. The formulae for a cylinder are

$$
\begin{aligned}
r(\theta) & = \begin{cases}\frac{a}{\sin \theta} & \text { if } \alpha \leq \theta \leq \pi-\alpha \\
\frac{c}{\cos \theta} & \text { otherwise }\end{cases} \\
r_{\theta} & = \begin{cases}-\frac{a \cos \theta}{\sin ^{2} \theta} & \text { if } \alpha \leq \theta \leq \pi-\alpha \\
-\frac{c \sin \theta}{\cos ^{2} \theta} & \text { otherwise }\end{cases}
\end{aligned}
$$

where $\alpha=\tan ^{-1}(a / c)$.

The aspect ratio for a cylinder is given by

$$
h=\frac{\sqrt{a^{2}+c^{2}}}{\min (a, c)} .
$$

\subsection{Numerical Precision}

This section begins with a brief examination of how numerical values are stored by computers, and how this leads to numerical problems in some cases. In the following, we refer solely to floating point numbers, which are those where the number is expressed as some value (a significand) multiplied by an exponent, which allows a large range of sizes to be represented. The standard precision offered is double precision, which contains 53 bits (equivalent to $\sim 16$ digits) of precision for the significand, and 11 bits for the exponent. Arbitrary precision methods allow for increasing the precision of the significand (and exponent) to arbitrary values, allowing the working precision to be set to suit the problem at hand. The cost for this is increased computation time and memory requirements, and potentially needing to modify software to incorporate arbitrary-precision methods.

The easiest way to demonstrate a loss of precision is to perform some addition or subtraction of numbers of similar sizes, where the correct result is small compared 


\section{Problematic Spheroids}

to the numbers. For example, consider the two numbers

$$
\begin{aligned}
& a=10^{10}+\pi \\
& b=10^{10} .
\end{aligned}
$$

We are able to easily see what the result of $a-b$ should be $(\pi)$, yet if we were to calculate this in some double-precision numerical software, we get a result 3.141592025756836, where the numbers in red italics differ from the correct digits. If we increase the difference in size of the numbers,

$$
\begin{aligned}
& a=10^{17}+\pi \\
& b=10^{17}
\end{aligned}
$$

then while the correct solution is unchanged, the numerical result is now $a-b=0$. This example shows the result losing accuracy, but the worst result that can be obtained is simply zero. However, there are other losses of precision that may produce much more incorrect results. As an example of this, consider

$$
\begin{aligned}
& a=10^{40} \times \frac{1}{3} \\
& b=10^{40} \times\left(1-\frac{2}{3}\right) .
\end{aligned}
$$

In this case, we can see that $a-b$ should be zero, yet when calculated amounts to $a-b=-6.04462909807315 \times 10^{23}$. As integration amounts to a numerical summation of many different numbers, it becomes obvious how that process may lead to a loss of precision. In the case of the EBCM integrals, the integrands are highly oscillatory, varying between large positive and negative values, leading to the possibility of cancellation problems.

A range of arbitrary-precision libraries are used in this work. The base library is the GNU Multiple Precision Arithmetic Library (GMP)[74]. On top of this, MPFR[75] is used to support floating-point numbers, and MPC[76] is used to add complex numbers. All of these libraries are used from the $\mathrm{C}$ programming language for the most part. However, they are used with $\mathrm{C}++$, when used in conjunction 
with the last library that we used, MPACK[77]. This library adds linear algebra capabilities, such as matrix inversion methods. The $\mathrm{C}$ and $\mathrm{C}++$ codes used are written as a mex file, so that MATLAB can call the functions. Most of these codes were written by myself as part of a short-term summer project preceding this thesis, but many modifications, including the matrix inversion capability, were added as part of this thesis.

When using arbitrary-precision code, it is possible to remove all precision issues, by simply increasing the working precision until the result converges within some desired precision. In this work, that desired precision is always double precision, as the results are truncated to double precision when they are transferred into MATLAB for analysis. In order to compare the relative agreement between two results, we introduce here the metric $\alpha$, defined as

$$
\alpha=-\log _{10}\left(\left|\frac{A-B}{B}\right|\right)
$$

where here $B$ is the value that is assumed correct (such as a value from arbitraryprecision calculations), and $A$ is the value that is being compared to it. $\alpha$ then represents the number of digits of agreement between $A$ and $B$. When $\alpha$ is infinity, $A$ and $B$ agree exactly (within the working precision, the maximum finite value of $\alpha$ is about 16 in double precision). As $A$ becomes large compared to $B, \alpha$ becomes negative when the order of magnitude of the two disagree. If $A$ becomes small (close to zero) compared to $B$, then $\alpha$ approaches zero. This metric $\alpha$ is used frequently in this chapter to evaluate the numerical performance of various methods.

\subsection{The Problem with Spheroids}

We start here with a demonstration of how the numerical problems for spheroids manifest. This is achieved by using the arbitrary-precision code to obtain the correct (converged to double precision) results, and comparing these to results calculated in double precision.

Here we provide three example calculations, for three different shapes, to em- 


\section{Problematic Spheroids}

phasise where the numerical problems appear. The three shapes are a prolate spheroid, a cylinder, and an offset sphere. We treat here shapes with a size parameter $x_{\max }=k_{1} r_{\max }=0.5$, an aspect ratio $h=2$ and a relative refractive index $s=1.5+0.02 i$.

We start by examining the extinction cross section of the three shapes for excitation with $\vec{E}$ along the long z-axis, as we increase the maximum multipole order $N$ being considered. In Fig. 5.1 is plotted the agreement in the extinction cross section, compared to the $N=41$ arbitrary-precision value. The agreement is plotted for calculations in both double and arbitrary precision. As can be seen in that figure, in the cases of the spheroid and the offset sphere, the number of digits of agreement $\alpha$ in the arbitrary precision results tends towards 16, indicating that the results are converging to the limits of the double precision the cross section is calculated in. However, for the double precision results, a different trend is apparent. For these two shapes, we can see an example of 'pseudo-convergence', where the results begin to converge towards the correct value, as for the arbitrary precision case, before diverging. For the cylinder, the results struggle to converge. The important difference between the cylinder, as opposed to the other two shapes, is that both the doubleand arbitrary-precision results for the cylinder follow the same path. Here, the failure to converge is not because of a loss of precision in the integration. Rather, we attribute this to a matrix inversion issue, or similar. The matrix inversion for all of these examples was performed in double precision. In the case of the spheroid and offset sphere, the arbitrary-precision results converge well, while it is only the double-precision results which exhibit problems. As the difference between the two is the precision used in the integration, this suggests a loss of precision is the cause for the problems for these shapes.

The cause of this behaviour is that for low $N$, both codes produce the same values for the integrals, and so behave the same. However, as $N$ increases, the integrals in the double precision case begin to produce incorrect results in the case of spheroids and offset spheres, and so the subsequent calculation of the extinction cross section will also be incorrect. It is possible to truncate the calculations to the point of best convergence (tested as relative convergence, as the fully-converged results will not be available as in this example). However, it would be preferable to be able to 


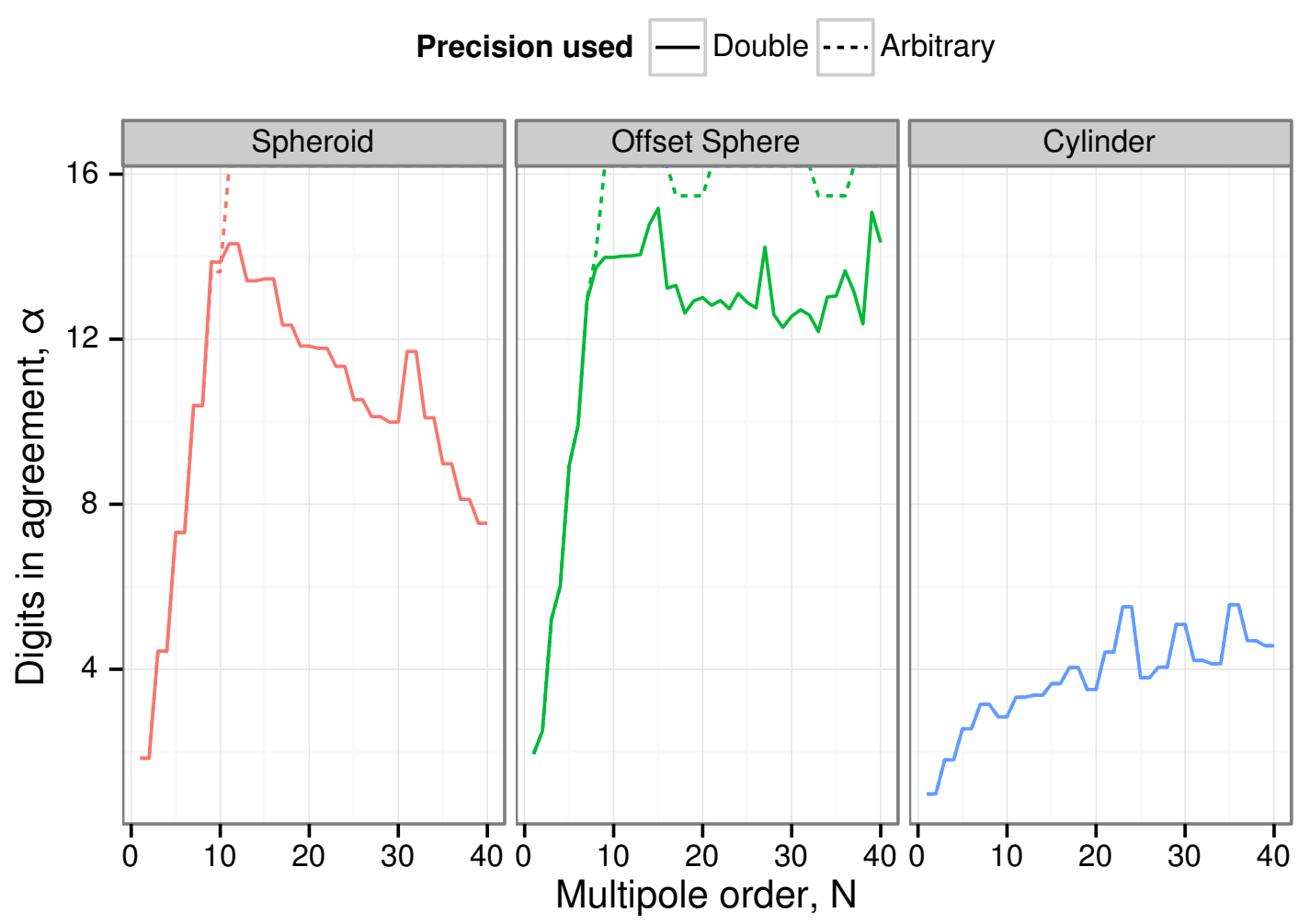

Figure 5.1: The number of digits in agreement with the $N=41$ result for the scattering cross section for a spheroid, offset sphere, and cylinder, with aspect ratio $h=2$, size parameter $x_{\max }=0.5$, and relative refractive index $s=1.5+0.02 i$. Results are shown for calculation of $\mathbf{P}$ and $\mathbf{Q}$ in double (solid lines) and arbitrary precision (dashed). The size of the inverted matrices for each point is $N$.

calculate the cross section without needing to worry about this divergence. Such a method is presented later.

In order to understand why the results begin to diverge, it is worth considering the behaviour of the integrands and the integrals in the calculation of $\mathbf{P}$ and $\mathbf{Q}$. We can see in Fig. 5.2(a)-(c) that the maximum integrand values for the three shapes do not vary with shape, but are different for different entries in the matrices. Figure 5.2 (d)(f) shows the manner in which integrals, calculated in arbitrary precision, change in size across the $Q$-matrix. It is clear here that for two of the shapes, the integral 


\section{Problematic Spheroids}

and the integrand behave differently. While for cylinders the behaviour of the two is the same, for the offset sphere and the spheroid, the scaling behaviour is radically different. While the integrand increases approaching the high- $n /$ low- $k$ corner of the matrix, for offset spheres the integral decreases, symmetrically with the high- $k /$ low$n$ corner, and tends towards a non-offset sphere (a sphere), for which all off-diagonal terms in this matrix would be zero. The failure of the integrals is demonstrated in Fig. 5.2(g)-(i), where the number of digits of agreement between double precision results and arbitrary precision results is shown. Here we can see that where the integrands are large compared to the correct integrals, there is a loss of precision in the results of the double precision integrals.

As Fig. 5.2 shows, the relative magnitude of the integral and the integrand varies in the cases of spheroids and offset spheres across the matrix, while for the cylinder, the integral and integrand scale the same. As this difference becomes larger, then the precision of the numerical results degrades. As can be seen in that figure, even in the case of the cylinder, where the magnitude of the integral is the same as the integrand, the summation of many terms leads to a slight drop in precision in some parts of the matrix, yet the error never becomes catastrophic.

In order to understand why some shapes behave differently to other shapes, we can look at the way the integrals and integrands scale with the size of the particle. This is shown for certain entries of the matrix $\mathbf{L}^{1}$ in Fig. 5.3. In all cases, the integrands exhibit the same behaviour as the cylinder integrals. The panels in the bottom row correspond to matrix entries that exhibit a loss of precision in Fig. 5.2 (g)-(i). In these cases, but not the top row (which corresponds to entries not showing a loss of precision in Fig. 5.2) we can see that the different shapes behave very differently. The integral considered here, $\mathbf{L}^{1}$, has the form

$$
L_{n k}^{1}=\int_{0}^{\pi} \mathrm{d} \theta x_{\theta} \tau_{n} d_{k} \chi_{n} \psi_{k}
$$

and if we expand the Bessel functions in a power series (details are found in Appendix $\mathrm{A}$ the leading-order term of this product is then $x^{k-n+2}$. Hence, by rescaling the particle that we model, we expect the integral to scale by this factor, $x^{k-n+2}$. As shown in Fig. 5.3. for small sizes the behaviour of the cylinder matches this expected 

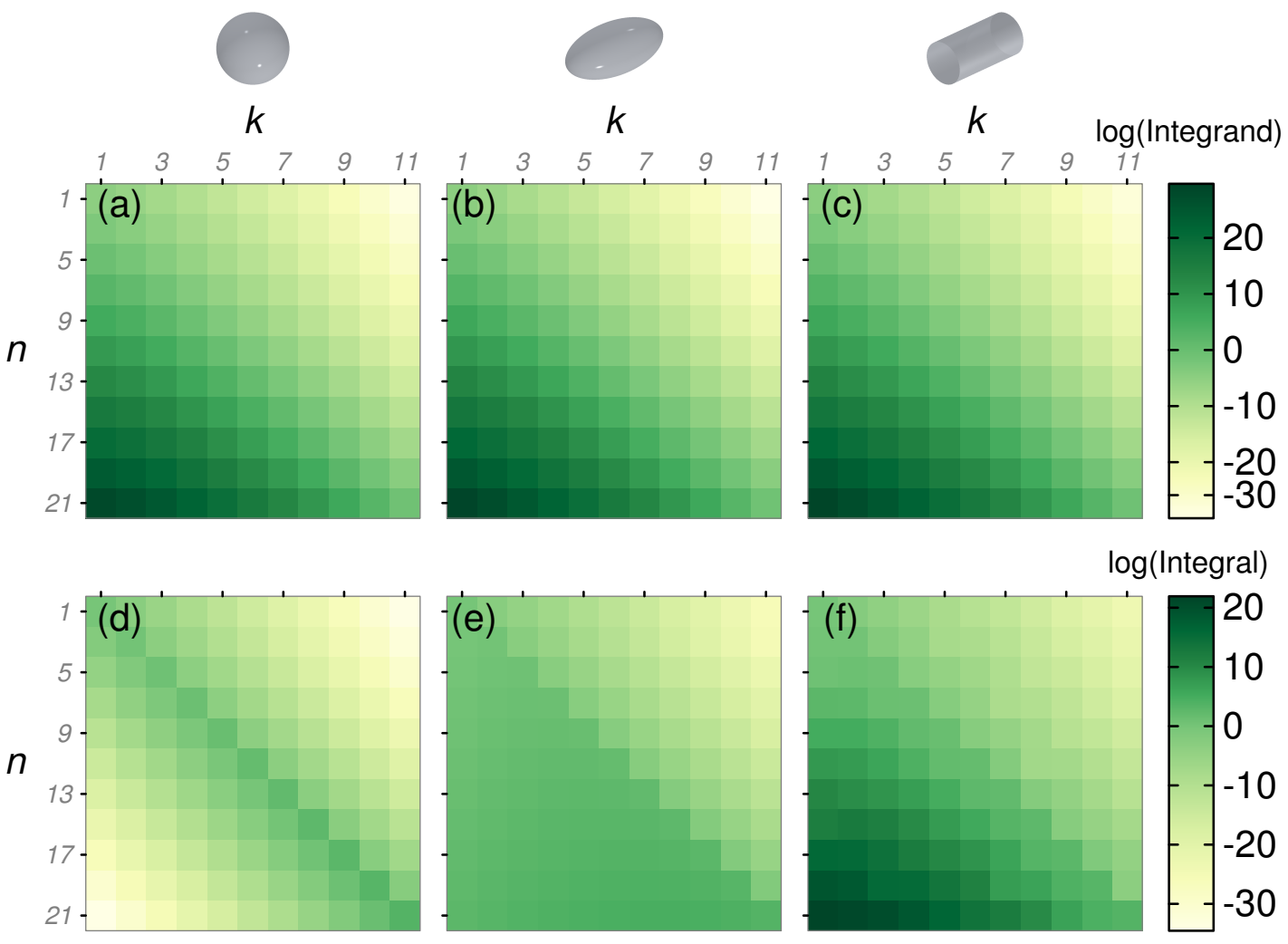

$\log ($ Integral)
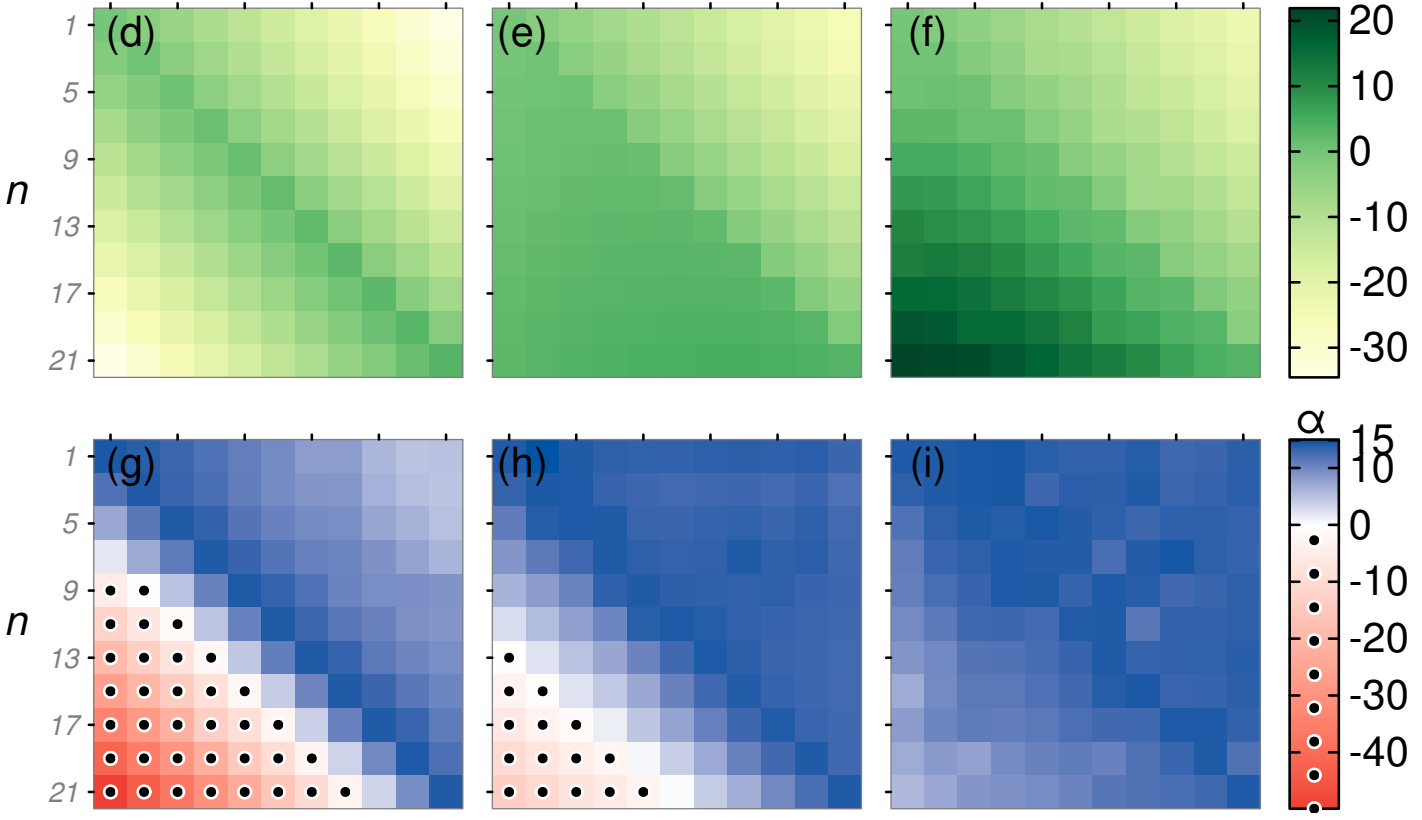

Figure 5.2: This shows the magnitude of the integrands for the three shapes in (a)(c). The integrals are shown in (d)-(f), and the cylinder behaves the same as the integrals. The offset sphere and cylinder show different behaviour. The number of digits correct $(\alpha)$ when calculated in double precision, compared to converged arbitrary-precision results, are given in (g)-(i). This figure only shows the odd-odd entries in $\mathbf{Q}^{22}$ for $m=1$, but the same results hold for other entries and matrices, and other $m$-values. Here black dots indicate that $\alpha<0$ (and so the order of magnitude is incorrect). 


\section{Problematic Spheroids}

behaviour, while the other two shapes behave differently in those cases where we observe a loss of precision. Once the sizes become larger, then the first term in the series expansion is no longer the only significant term, and all of the shapes behave similarly. It was only possible to carry out this study using the arbitrary-precision codes. In double precision, the magnitude of the integrals cannot be evaluated for the spheroid and offset sphere where they differ in behaviour from a cylinder.

Based on the leading term in the power series, we can easily see how the integrand will scale with size of the scatterer. These go like $x^{k-n+2}$ for the integral $L_{n k}^{1}$. However, for the spheroid and offset sphere, we can see that the scaling is not like this: these leading terms in the integration do not appear to contribute. From this, we suppose that some leading terms in the power series integrate to zero,

$$
\int_{0}^{\pi} \mathrm{d} \theta f(\theta) x^{p}=0
$$

for some appropriate $f(\theta)$ describing the angular terms, and for some term $x^{p}$ from the power series. However, these terms are also the largest in the integrands, and so lead to severe loss of precision when adding up a series of numbers that are large compared to their sum. This results in calculating integrals where the value computed is 16 orders of magnitude smaller than the maximum value, and otherwise bears no relation to the correct result.

The solution to this is conceptually simple. We should simply integrate the part of the power series that integrates to a non-zero value, and the remaining terms make no contribution and may be safely discarded. Here, this solution is broken into two parts. The first is a formal derivation of exactly which terms integrate to zero in these integrals, and the second part is a method that is able to generate the required parts of the integrand in a numerically stable manner. 


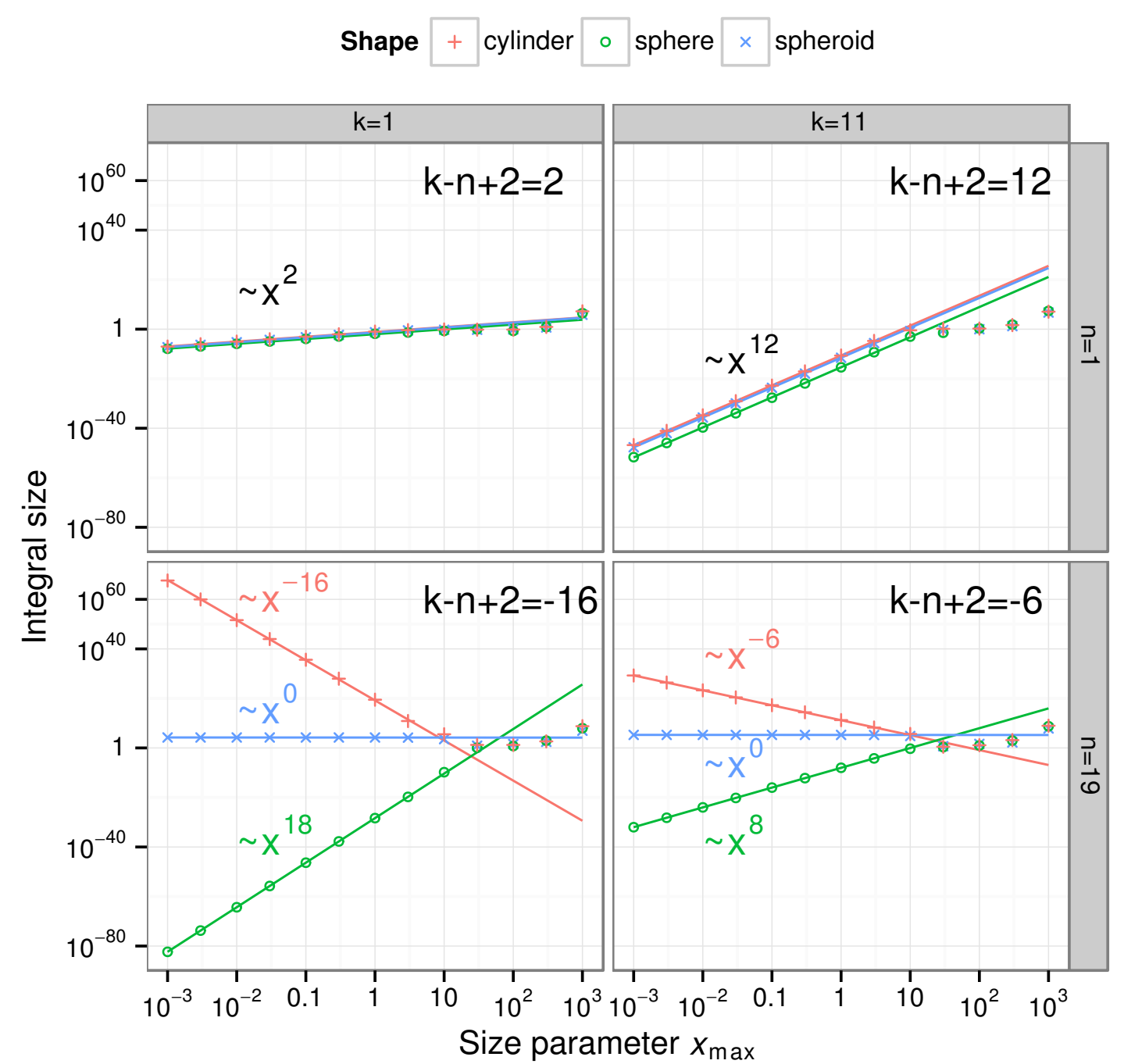

Figure 5.3: This demonstrates how the integrals $L_{n k}^{1}$ scale with the particle size, for the three shapes cylinder, offset sphere, and spheroid. This is shown as a function of $n$ and $k$ and the size of the particles. The scaling of all of the integrands follows the cylinder. This is for $m=1$, and the integrals are calculated in arbitrary precision, with a precision and number of integration points such that the results have converged within double precision. 


\subsection{Proof that Spheroid Integrals Contain Terms Integrating to Zero}

First we present a detailed demonstration of which terms in the integrals make no contribution to the result (that is, integrate identically to zero). This is divided into sections for each of the integrals.

\subsubsection{Necessary Relations}

Here we outline some important relations needed for these proofs. We begin by defining the notation

$$
\mathcal{P}_{N}(\cos \theta)
$$

to mean a polynomial in $\cos \theta$ of degree $N$ or less. We also make use of the ability to use the set of Legendre polynomials $P_{n}^{0}(X)$, with $0 \leq n \leq N$ as a basis of the vector space $\mathbb{R}_{N}[X]$, denoting here real polynomials of degree $N$.

We make use of a formula by Gaunt which expresses the integral of the product of three associated Legendre functions [78]. In our case, we are interested only in the special case of when the integral is zero, and the third associated Legendre function is a Legendre polynomial, that is

$$
\begin{aligned}
& \int_{0}^{\pi} \mathrm{d} \theta P_{n}^{m}(\cos \theta) P_{k}^{m}(\cos \theta) P_{p}^{0}(\cos \theta) \sin \theta=0 \\
& \text { if }\left\{\begin{array}{c}
|n-k|>p \\
\text { or } \\
n+k+p \text { is odd. }
\end{array}\right.
\end{aligned}
$$

This may be written in terms of $d_{n}$ as

$$
\begin{aligned}
& \int_{0}^{\pi} \mathrm{d} \theta d_{n}(\theta) d_{k}(\theta) \mathcal{P}_{p}(\cos \theta) \sin \theta=0 \\
& \text { if }|n-k|>p
\end{aligned}
$$


The proofs then consist mainly of placing the integrals into a form as in this equation, and seeing that the terms that we have observed not to contribute all integrate identically to zero.

The other important step, and which is what makes the spheroid special, is to obtain some expressions about the spheroid geometry. It is these relations on $x \equiv x(\theta)$ which provide all of the shape-dependent information in the integrals. Hence, this is what causes the spheroids (and offset sphere) to behave in a manner very different to other shapes. The geometry is defined as in Section 5.1.1, and we may write the expressions

$$
\begin{aligned}
\frac{x_{\theta}}{x^{3}} & =\frac{a^{2}-c^{2}}{k_{1}^{2} a^{2} c^{2}} \sin \theta \cos \theta \\
\frac{1}{x^{2}} & =\frac{1}{k_{1}^{2} a^{2}}+\frac{a^{2}-c^{2}}{k_{1}^{2} a^{2} c^{2}} \cos ^{2} \theta \\
\frac{\sin \theta}{x^{2}} & =\frac{\sin \theta}{k_{1}^{2} a^{2}}+\frac{x_{\theta} \sin \theta}{x^{3}} .
\end{aligned}
$$

and by combining these, it is also possible to obtain

$$
\begin{array}{ll}
\frac{x_{\theta}}{x^{2 q+3}}=\cos \theta \sin \theta\left(\alpha+\beta \cos ^{2} \theta\right)^{q} & =\sin \theta \mathcal{P}_{2 q+1}(\cos \theta) \\
\frac{\sin \theta}{x^{2 q+2}}=\sin \theta\left(\alpha+\beta \cos ^{2} \theta\right)^{q+1} & =\sin \theta \mathcal{P}_{2 q+2}(\cos \theta)
\end{array}
$$

where we define

$$
\begin{aligned}
& \alpha=\frac{1}{k_{1}^{2} a^{2}} \\
& \beta=\frac{a^{2}-c^{2}}{k_{1}^{2} a^{2} c^{2}} .
\end{aligned}
$$

While the exact values for $\alpha$ and $\beta$ are provided in this instance, here we introduce a notational nicety whereby the values of variables styled in Greek letters are not specified or important. We exclude from this convention the functions $\pi$ and $\tau$, the Bessel functions $\chi$ and $\psi$, and the angle $\theta$. None of the variables used diverge to infinity, and as we are interested in showing when terms are zero, the exact value of any prefactors is of no importance. 


\section{Problematic Spheroids}

It is important to note how the angular functions come into this. The function $d_{n}=C_{n m} P_{n}^{m}(\cos \theta)$ is simply an associated Legendre function, with $C_{n m}$ defined as in Appendix B, and $d_{n}$ as used here has an implied index $m$, so does not need any relation. The other angular function, $\tau_{n}=\tau_{n m}(\theta)=\frac{\mathrm{d}}{\mathrm{d} \theta} d_{n}$, may be rewritten in terms of $d_{n}$ as

$$
\sin \theta \tau_{n}=-\sqrt{n^{2}-m^{2}} d_{n-1}+n d_{n}
$$

This allows us to simply rewrite Eq. (5.19) in terms of $\tau_{n}$, as

$$
\begin{aligned}
& \int_{0}^{\pi} \mathrm{d} \theta \sin \theta \tau_{n}(\theta) d_{k}(\theta) \mathcal{P}_{p}(\cos \theta) \sin \theta=0 \\
& \text { if } p \leq|n-k|-2 .
\end{aligned}
$$

\subsubsection{Integrals $\mathrm{L}^{1}$ and $\mathrm{L}^{2}$}

For the integral $L_{n k}^{1}$, we have the expression

$$
L_{n k}^{1}=\int_{0}^{\pi} \mathrm{d} \theta \sin \theta x_{\theta} \tau_{n} d_{k} \chi_{n} \psi_{k}
$$

In the case of spheroids, this integral is zero (by parity) whenever $n+k$ is odd, hence we shall assume here that $n+k$ is even. We are also able to expand the product of the Bessel functions as

$$
\chi_{n} \psi_{k}=x^{k-n+1} \sum_{q=0}^{\infty} \nu_{n k q}(s) x^{2 q},
$$

and so we may write

$$
L_{n k}^{1}=\sum_{\substack{p=k-n+1 \\ p \text { odd }}}^{\infty} \mu_{n k p}(s) L_{n k p}^{1}
$$


where

$$
L_{n k p}^{1}=\int_{0}^{\pi} \mathrm{d} \theta \sin \theta x_{\theta} x(\theta)^{p} \tau_{n}(\theta) d_{k}(\theta)
$$

Written in this way, each integral $L_{n k p}^{1}$ contains only one power of $x$, and based on our observations of the scaling of the integrals, the lower orders should all integrate to zero. We observed anomalous scaling only in the part of the matrix where $n \geq k+4$ (that is, below and not immediately adjacent to the diagonal), hence we shall only consider cases where $n \geq k+4$. Assuming that $p \leq-3$, using Eq. (5.24), and defining the non-negative integer $q=-(p+3) / 2$ we may rewrite the integral as

$$
L_{n k p}^{1}=\int_{0}^{\pi} \mathrm{d} \theta \sin ^{2} \theta \mathcal{P}_{2 q+1}(\cos \theta) \tau_{n}(\theta) d_{k}(\theta)
$$

By using our modified Gaunt's formula, Eq. (5.29), we can immediately see that this integral is zero for $2 q+1 \leq|n-k|-2$, or equivalently, as $|n-k|$ is even, this may be written $|n-k| \geq 2 q+4$, which is also equivalent to $k-n+1 \leq p$. Through the scaling studies, it is expected that the integrals will be zero whenever $k-n+1 \leq p \leq-3$. This agrees with our conclusion above. Note that for $p \geq-1$, then this proof is not valid, which amounts to the integrals being non-zero, as expected.

For $\mathbf{L}^{2}$, we have

$$
L_{n k}^{2}=\int_{0}^{\pi} \mathrm{d} \theta \sin \theta x_{\theta} d_{n} \tau_{k} \chi_{n} \psi_{k}
$$

where the only difference from $\mathbf{L}^{1}$ is the indices on $d$ and $\tau$. However, as these indices only appear as $|n-k|$ in the condition of Eq. (5.29), they are interchangeable, and the results are the same as for $\mathbf{L}^{1}$. This is expected, as these two integrals were found to scale the same way. 


\subsubsection{Integrals $\mathrm{K}^{1}$ and $\mathrm{K}^{2}$}

The integral $\mathbf{K}^{1}$ is

$$
K_{n k}^{1}=\int_{0}^{\pi} \mathrm{d} \theta m d_{n} d_{k} x_{\theta} \chi_{n} \psi_{k}^{\prime}
$$

Along the same lines as for $\mathbf{L}^{1}$, we may express the product of the Bessel functions as

$$
\chi_{n} \psi_{k}^{\prime}=x^{k-n} \sum_{q=0}^{\infty} \alpha_{n k q}(s) x^{2 q},
$$

where here $n+k$ is odd, because for the entries where $n+k$ is even, $K_{n k}^{1}$ is zero by parity. Thus, we may define

$$
K_{n k p}^{1}=\int_{0}^{\pi} \mathrm{d} \theta x_{\theta} x(\theta)^{p} d_{n}(\theta) d_{k}(\theta)
$$

where

$$
K_{n k}^{1}=\sum_{\substack{p=k-n \\ p \text { odd }}}^{\infty} \nu_{n k p}(s) K_{n k p}^{1} .
$$

Based on observations of the scaling behaviour of the integrals, we expect that $K_{n k p}^{1}$ is zero when $k-n \leq p \leq-3$. We are able to rewrite the $x$-terms, to get

$$
K_{n k p}^{1}=\int_{0}^{\pi} \mathrm{d} \theta \sin \theta \mathcal{P}_{2 q+1}(\cos \theta) d_{n}(\theta) d_{k}(\theta)
$$

where $q=-(p+3) / 2$ is a non-negative integer. Thus, from direct application of Eq. (5.19), $K_{n k p}^{1}=0$ when $|n-k|-1 \geq 2 q+1$. Given that $|n-k|$ is odd, this is the same as $|n-k| \geq 2 q+3$, which is also equivalent to $k-n \leq p !^{1}$ Thus, all of the terms that we need to integrate to zero do integrate to zero.

\footnotetext{
${ }^{1}$ Of interest there is that terms in, for example, $p=k-n-2$, if they had a non-zero coefficient, would not integrate to zero. It is only exactly the terms that we want to integrate to zero that do integrate to zero.
} 
In order to show this for $\mathbf{K}^{2}$, only one small change is required. In this case, we have the integral

$$
K_{n k}^{2}=\int_{0}^{\pi} \mathrm{d} \theta m d_{n} d_{k} x_{\theta} \chi_{n}^{\prime} \psi_{k}
$$

and we may express

$$
\chi_{n}^{\prime} \psi_{k}=x^{k-n} \sum_{q=0}^{\infty} \beta_{n k q}(s) x^{2 q}
$$

which is the same as Eq. (5.37) except for the coefficients, and so the rest of the results are identical.

\subsubsection{Integrals $\mathrm{L}^{3}$ and $\mathrm{L}^{4}$}

We might hope that the proofs for the last two integrals would be as simple as for the previous four. As we shall see, they are not quite as straightforward.

We shall start by looking at $\mathbf{L}^{3}$. The expression for this is

$$
L_{n k}^{3}=\int_{0}^{\pi} \mathrm{d} \theta \sin \theta d_{k} \psi_{k}^{\prime}\left[x_{\theta} \tau_{n} \chi_{n}^{\prime}-n(n+1) d_{n} \chi_{n}\right]
$$

It may appear tempting to split this into two terms, and work on each one separately. While this approach works to show that almost all of the required terms integrate to zero for each integral, the leading term in both cases, as we shall see, does not integrate to zero. Rather, the leading terms make no contribution because the two leading terms cancel each other. Thus, in this proof, we shall need to consider the exact coefficients of the two terms in order to demonstrate this.

For $\mathbf{L}^{3}$, we have $n+k$ even, as for $n+k$ odd entries the integrals are identically zero by parity. 


\section{Problematic Spheroids}

Firstly, we may write the integral as

$$
L_{n k}^{3}=\sum_{\substack{p=k-n-1 \\ p \text { odd }}}^{\infty} L_{n k p}^{3}
$$

where

$$
L_{n k p}^{3}=\int_{0}^{\pi} \mathrm{d} \theta \sin \theta d_{k}\left[\alpha_{n k p}(s) x_{\theta} x^{p} \tau_{n}-n(n+1) \beta_{n k p}(s) x^{p+1} d_{n}\right]
$$

Note that the two terms scale the same, as $x_{\theta} x^{p}$ scales like $x^{p+1}$. The difference in the two coefficients $\alpha_{n k p}(s)$ and $\beta_{n k p}(s)$ is the difference in the leading term of $\chi_{n}^{\prime}$ and $\chi_{n}$, which is

$$
\frac{\left.\chi_{n}^{\prime}\right|_{\text {leading term }}}{\left.\chi_{n}\right|_{\text {leading term }}}=-n .
$$

Thus, we may rewrite for the leading term

$$
\left.L_{n k p}^{3}\right|_{p=k-n-1}=-\int_{0}^{\pi} \mathrm{d} \theta d_{k} \sin \theta \beta_{n k p}(s)\left[n x_{\theta} x^{p} \tau_{n}+n(n+1) x^{p+1} d_{n}\right],
$$

and using Eq. (5.23) this may be rewritten as

$$
\left.L_{n k p}^{3}\right|_{p=k-n-1}=-\int_{0}^{\pi} \mathrm{d} \theta d_{k} n x^{p+3} \beta_{n k p}(s)\left[\sin \theta \frac{x_{\theta}}{x^{3}} \tau_{n}+\alpha(n+1) \sin \theta d_{n}+(n+1) d_{n} \cos \theta \frac{x_{\theta}}{x^{3}}\right] .
$$

We may use one of the angular function relations, Eq. (B.15) to write

$$
\left.L_{n k p}^{3}\right|_{p=k-n-1}=-\int_{0}^{\pi} \mathrm{d} \theta d_{k} n x^{p+3} \beta_{n k p}(s)\left[\frac{x_{\theta}}{x^{3}} d_{n+1} \sqrt{(n+1)^{2}-m^{2}}+\alpha(n+1) \sin \theta d_{n}\right],
$$


which may be split into two integrals,

$\left.L_{n k p}^{3}\right|_{p=k-n-1}=\int_{0}^{\pi} \mathrm{d} \theta n x_{\theta} x^{p} \beta_{n k p}^{\prime}(s) d_{n+1} d_{k}+\int_{0}^{\pi} \mathrm{d} \theta \sin \theta n x^{p+3} \beta_{n k p}^{\prime \prime}(s)(n+1) d_{n} d_{k}$.

where $\beta_{n k p}^{\prime}(s)=-\beta_{n k p}(s) \sqrt{(n+1)^{2}-m^{2}}$ and $\beta_{n k p}^{\prime \prime}(s)=-\beta_{n k p}(s) \alpha$.

The first of those integrals may be expressed as

$$
\int_{0}^{\pi} \mathrm{d} \theta n x_{\theta} x^{p} \beta_{n k p}^{\prime}(s) d_{n+1} d_{k}=\int_{0}^{\pi} \mathrm{d} \theta \sin \theta n \mathcal{P}_{2 q+1}(\cos \theta) \beta_{n k p}^{\prime}(s) d_{n+1} d_{k}
$$

where $q=-(p+3) / 2$, and we made use of Eq. (5.24). Thus, we can see that this integral is zero when $2 q+1 \leq|n+1-k|-1$, equivalent to $-p-2 \leq n-k$. In this case (of the leading term), $p=k-n-1$, so $n-k-1 \leq n-k$ is trivially true, and the integral is zero.

The second of the integrals may be written using Eq. (5.25) as

$$
\int_{0}^{\pi} \mathrm{d} \theta \sin \theta x^{p+3} \beta_{n k p}^{\prime \prime}(s) n(n+1) d_{n} d_{k}=\int_{0}^{\pi} \mathrm{d} \theta \sin \theta \mathcal{P}_{2 q+2}(\cos \theta) \beta_{n k p}^{\prime \prime}(s) n(n+1) d_{n} d_{k}
$$

where $q=-(p+5) / 2$. This is then zero when $2 q+2 \leq|n-k|-1$, or $-p-3 \leq$ $|n-k|-1$. Given the value for $p$ being used here, this simplifies to $n-k-1 \leq n-k$, which is again true, and so this integral is zero.

We have established that the leading order in $L_{n k}^{3}$ integrates to zero. For the other terms, we divide the integral into the two terms,

$$
\begin{aligned}
L_{n k}^{31} & =\int_{0}^{\pi} \mathrm{d} \theta \sin \theta x_{\theta} \tau_{n} d_{k} \chi_{n}^{\prime} \psi_{k}^{\prime} \\
L_{n k}^{32} & =-n(n+1) \int_{0}^{\pi} \mathrm{d} \theta \sin \theta d_{n} d_{k} \chi_{n} \psi_{k}^{\prime} .
\end{aligned}
$$




\section{Problematic Spheroids}

Looking first at $L_{n k}^{31}$, we may write the product of the Bessel functions as

$$
\chi_{n}^{\prime} \psi_{k}^{\prime}=x^{k-n-1} \sum_{q=0}^{\infty} \gamma_{n k q}(s) x^{2 q}
$$

and hence

$$
L_{n k}^{31}=\sum_{\substack{p=k-n-1 \\ p \text { odd }}}^{\infty} \iota_{n k p}(s) L_{n k p}^{31}
$$

where

$$
L_{n k p}^{31}=\int_{0}^{\pi} \mathrm{d} \theta \sin \theta \tau_{n} d_{k} x_{\theta} x^{p}
$$

By defining the non-negative index $q=-(p+3) / 2$ and using Eq. (5.24), we arrive at

$$
L_{n k p}^{31}=\int_{0}^{\pi} \mathrm{d} \theta \sin ^{2} \theta \tau_{n} d_{k} \mathcal{P}_{2 q+1}(\cos \theta)
$$

which by Eq. (5.29) is zero whenever $2 q+1 \leq|n-k|-2$. In terms of $p$, this equates to $-p \leq|n-k|$. The largest value of $-p$ is $n-k+1$, which is not less than $|n-k|$, however the next value of $-p$ is $n-k-1$, which is less than $|n-k|$. We do not expect that the first term would fulfil the condition to integrate to zero, but as already shown, that term cancels out with the first term from $L_{n k}^{32}$. So, the terms that integrate to zero in $L_{n k}^{31}$ are when $n-k-1 \leq p \leq-3$, which matches with our expectations based on the scaling study.

Considering $L_{n k}^{32}$, we write the product of the Bessel functions as

$$
\chi_{n} \psi_{k}^{\prime}=x^{k-n} \sum_{q=0}^{\infty} \delta_{n k q}(s) x^{2 q} .
$$


Thus,

$$
L_{n k}^{32}=\sum_{\substack{p=k-n-1 \\ p \text { odd }}}^{\infty} \rho_{n k p}(s) L_{n k p}^{32}
$$

where

$$
L_{n k p}^{32}=-n(n+1) \int_{0}^{\pi} \mathrm{d} \theta \sin \theta d_{n} d_{k} x^{p+1} .
$$

We again define the non-negative integer $q=-(p+3) / 2$, so that by using Eq. (5.25) we have

$$
L_{n k p}^{32}=-n(n+1) \int_{0}^{\pi} \mathrm{d} \theta \sin \theta d_{n} d_{k} \mathcal{P}_{2 q+2}(\cos \theta)
$$

which is zero when $2 q+2 \leq|n-k|-1$, or equivalently $-p \leq|n-k|$, which is the same as the result for $L_{n k}^{31}$, and hence all of the terms except the leading term integrate to zero. The leading term here, as we have seen, cancels with the leading term of $L_{n k}^{31}$, and so all of the terms which we have observed making no contribution to the integrals do integrate identically to zero.

The only remaining integral to consider is $\mathbf{L}^{4}$. While a similar analysis could be applied to $\mathbf{L}^{4}$, this is not necessary. As shown in Section 4.1 .4 in Eq. 4.44, $\mathbf{L}^{4}$ may be expressed as a linear combination of $\mathbf{L}^{1}, \mathbf{L}^{2}$ and $\mathbf{L}^{3}$, and thus the first contributing order in $\mathbf{L}^{4}$ is simply the first order to contribute out of the other integrals.

\subsection{Inversion of the Correct Matrices}

It has been the focus of some works to improve the accuracy of the EBCM by improving the inversion used to calculate $\mathbf{T}$ from $\mathbf{P}$ and $\mathbf{Q}$. While this will improve the method in general, in the case of spheroids normal inversion routines provide adequate accuracy if the correct $\mathbf{Q}$ is used. Some of the suggested improvements to the inversion implement an improved inversion routine, for example making use of 


\section{Problematic Spheroids}

Gaussian elimination[79], Gaussian elimination with back-substitution [63, or LUfactorisation [65]. Other methods do the integration and inversion in extended precision (in this case, quad precision, or 113 bits of precision, compared to 53 bits for double precision), and observe an increased range of convergence 64]. Here, we will demonstrate that this improvement may be obtained with double-precision inversion, if the correct $Q$-matrix is used, and that it is not the inversion step that is crucial for the case of spheroids.

In order to determine how accurate a result is, we make use of the arbitrary precision code, modified to use the $\mathrm{C}++$ library MPACK[77], which is able to perform matrix inversion in arbitrary precision. This provides a good value against which we may compare the other methods. Here, the two different methods were used to calculate $\mathbf{P}$ and $\mathbf{Q}$. Then, the inversion was performed in double precision, and compared to the result where the matrices and the inversion were calculated in arbitrary precision. The results are shown in Fig. 5.4, and demonstrate that there is very good agreement even when the inversion is in double precision, so long as the initial matrices are calculated correctly.

This exemplifies the value in correctly calculating $\mathbf{Q}$, and demonstrates that it is not necessary for reasons of numerical accuracy to focus on the inversion step (this applies to spheroids, and is not true of other shapes). Of course, for speed or memory requirements, it might still be desirable to use different inversion schemes.

It is also possible to consider the effect of truncation on the precision of elements in $\mathbf{T}$. Some parity considerations aside, each element of $\mathbf{T}$ depends on the values of all elements of $\mathbf{P}$ and $\mathbf{U} \mathbf{2}^{2}$ However, as it is intractable to calculate and then invert an infinite matrix (or to perform either of those steps individually), the matrix is truncated before inversion. The assumption here is that values with high $n$ or $k$ make a negligible contribution to the earlier entries, especially those around $n=k=1$, as it is these early entries with typically contribute the most to the final results.

It is worth a brief mention of the methods used to invert the matrices. We use MATLAB, and divide the $T$-matrix up into two matrices, based on the parity of the

\footnotetext{
${ }^{2}$ For axisymmetric particles this is restricted to only values with the same $m$, and for particles with a plane of symmetry only values with appropriate parity of $n+k$.
} 

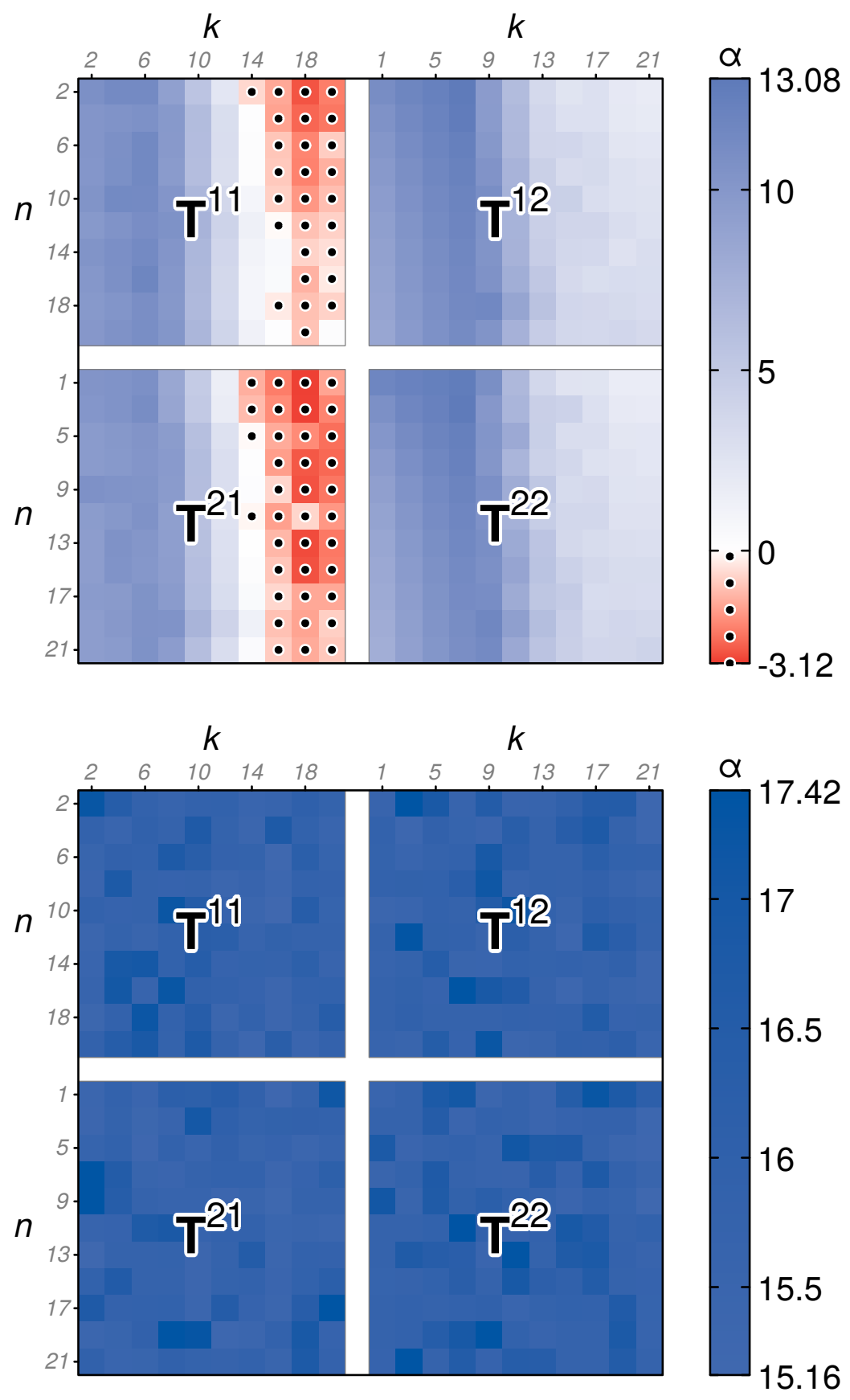

Figure 5.4: Number of correct digits of the $T$-matrix after inversion in double precision, after $\mathbf{P}$ and $\mathbf{Q}$ were calculated in (top) double and (bottom) arbitrary precision. This is relative to inversion also being carried out in arbitrary precision. The particle here is a prolate spheroid of aspect ratio $h=2$, size parameter $x_{\max }=1$, with relative refractive index $s=1.5+0.02 i$. Only $m=1$ is considered here. This shows only one parity of each $n, k$, for each block of the matrix, as indicated by the axis labels, though the other entries behave similarly. 
5 Problematic Spheroids

indices [6]. These two matrices are

$$
\begin{aligned}
& \mathbf{T}^{e o}=\left(\begin{array}{ll}
\mathbf{T}_{e e}^{11} & \mathbf{T}_{e o}^{12} \\
\mathbf{T}_{o e}^{21} & \mathbf{T}_{o o}^{22}
\end{array}\right) \\
& \mathbf{T}^{o e}=\left(\begin{array}{ll}
\mathbf{T}_{o o}^{11} & \mathbf{T}_{o e}^{12} \\
\mathbf{T}_{e o}^{21} & \mathbf{T}_{e e}^{22}
\end{array}\right)
\end{aligned}
$$

where the subscript $e_{e}$ indicates that the $n$-index of the matrix takes even values, and the $k$-index takes odd values. The same may be applied to $\mathbf{P}$ and $\mathbf{Q}$ also. Using this notation,

$$
\begin{aligned}
& \mathbf{T}^{\mathbf{e o}}=-\mathbf{P}^{\mathbf{e o}}\left(\mathbf{Q}^{\mathbf{e o}}\right)^{-1} \\
& \mathbf{T}^{\mathbf{o e}}=-\mathbf{P}^{\mathbf{o e}}\left(\mathbf{Q}^{\mathbf{o e}}\right)^{-1}
\end{aligned}
$$

These two parts each relate half of the field coefficients, as

$$
\begin{aligned}
\left(\begin{array}{c}
\vec{c}_{e} \\
\vec{d}_{o}
\end{array}\right) & =\mathbf{T}^{e o}\left(\begin{array}{c}
\vec{a}_{e} \\
\vec{b}_{o}
\end{array}\right) \\
\vec{a}_{e} & =\left(\begin{array}{c}
a_{2} \\
a_{4} \\
\vdots
\end{array}\right)
\end{aligned}
$$

and similarly for the other parity.

The inversion of these two equations is then carried out by block inversion scheme [79 81, using a LU factorisation, obtained by Gaussian elimination with partial pivoting. In MATLAB, this corresponds to the mrdivide operation, also denoted /. 
The block inversion is carried out in the following steps,

$$
\begin{aligned}
\mathbf{F}_{1} & =\mathbf{I} / \mathbf{Q}^{11} \\
\mathbf{G}_{1} & =\mathbf{P}^{11} \mathbf{F}_{1} \\
\mathbf{G}_{3} & =\mathbf{P}^{21} \mathbf{F}_{1} \\
\mathbf{G}_{5} & =\mathbf{Q}^{21} \mathbf{F}_{1} \\
\mathbf{F}_{2} & =\mathbf{I} /\left[\mathbf{Q}^{22}-\mathbf{G}_{5} \mathbf{Q}^{12}\right] \\
\mathbf{G}_{2} & =\mathbf{P}^{22} \mathbf{F}_{2} \\
\mathbf{G}_{4} & =\mathbf{P}^{12} \mathbf{F}_{2} \\
\mathbf{G}_{6} & =\mathbf{Q}^{12} \mathbf{F}_{2} \\
\mathbf{T}^{12} & =\mathbf{G}_{1} \mathbf{G}_{6}-\mathbf{G}_{4} \\
\mathbf{T}^{22} & =\mathbf{G}_{3} \mathbf{G}_{6}-\mathbf{G}_{2} \\
\mathbf{T}^{11} & =\mathbf{G}_{1}-\mathbf{T}^{12} \mathbf{G}_{5} \\
\mathbf{T}^{21} & =\mathbf{G}_{3}-\mathbf{T}^{22} \mathbf{G}_{5} .
\end{aligned}
$$

Here, for efficiency, this is carried out separately for $\mathbf{T}^{e o}$ and $\mathbf{T}^{o e}$. In our tests, this method does not result in a significant loss of precision, and was the method used in Fig. 5.4 .

\subsection{Spheroid Integral Forms}

Given our understanding of the cause of the loss of precision in the case of spheroids, we can now consider how to calculate the integrals without a loss of precision. Conceptually, the solution is simple. As the loss of precision is caused by dominant terms in the integrals which should make no contribution to the result, then the integrals should be calculated without making use of those terms.

In this section, we present the necessary relations to calculate the integrals in the case of spheroids. Similar work has not been done in the case of offset spheres, owing to the availability of Mie theory to calculate results for spherical scatterers, and the ability to spatially offset the results, which renders such effort unrewarding. 


\section{Problematic Spheroids}

The loss of precision for spheroids occurs only in the matrix $\mathbf{U}$ (and $\mathbf{Q}$, which is obtained from $\mathbf{U}$, but not $\mathbf{P}$ ). Hence, in this chapter we make use of the Bessel function products like $\chi_{n} \psi_{k}$, and not terms like $\psi_{n} \psi_{k}$, for which there are not the same numerical problems.

\subsubsection{Basic Idea}

To illustrate the underlying idea behind our approach, we define operators $\mathcal{P}^{+}$and $\mathcal{P}^{-}$such that for some function $f=f(x)$,

$$
\mathcal{P}^{+} f+\mathcal{P}^{-} f=f
$$

and where $\mathcal{P}^{+} f$ contains only the components of $f$ with non-negative powers of $x$, such that if

$$
f(x)=\frac{1}{x^{2}}+\frac{1}{x}+1+x^{2}+\ldots
$$

then

$$
\mathcal{P}^{+} f(x)=1+x+x^{2}+\ldots
$$

Our solution to the problem with spheroids therefore involves rewriting the integrals in a form such that

$$
L_{n k}^{5}=\int_{0}^{\pi} \mathrm{d} \theta \sin \theta\left(n(n+1) d_{n} \tau_{k}-k(k+1) \tau_{n} d_{k}\right) \frac{x_{\theta}}{x} \mathcal{P}^{+}\left[x \chi_{n} \psi_{k}\right]
$$

with similar expressions for the other integrals. Note that if $\mathcal{P}^{-}$was used here instead then the result would be zero, as the components with negative powers of $x$ integrate identically to zero. Here, $x_{\theta}$ exhibits the same scaling behaviour as $x$, and we have inserted a factor of $x$ into our positive-part operator to increase all terms by one order, so that we select terms of non-negative power, and not terms with powers at least -1 . 
We therefore define the function

$$
F_{n k}(s, x)=x \chi_{n}(s) \psi_{k}(s x)
$$

and for convenience define the general notation

$$
\begin{aligned}
f^{+} & \equiv \mathcal{P}^{+} f \\
f^{-} & \equiv \mathcal{P}^{-} f .
\end{aligned}
$$

This part of the solution is easy. The challenging part is to calculate the values of $F^{+}$(referred to as the 'positive part' for simplicity) in a numerically-stable way. It is worth noting that it is not stable to calculate $F^{+}=F-F^{-}$, as in general $F^{+}$is small compared to $F, F^{-}$and so there will be a loss of precision in that calculation. In fact, we must calculate $F^{+}$without making use of the value of $F^{-}$ in the calculation.

\subsubsection{Series Method}

The conceptually-simplest method to calculate $F^{+}$is by series expansion. There have been some hints in the literature as to how to implement this, yet no general solution has been provided[72, 73]. The series expansion of the functions $\chi$ and $\psi$ are given in Appendix A. We may write the series as

$$
F_{n k}^{+}=\left(x \chi_{n}(x) \psi_{k}(s x)\right)^{+}=\sum_{q=q_{\min }}^{\infty} c_{q} x^{2 q+k-n+2}
$$

for $q_{\min }=(n-k) / 2-1$, and the calculation of the values of $c_{q}$ is the substantial focus of this section.

The first, most obvious approach is to directly calculate the coefficients in the sum. By making use of the series expansion of the Riccati-Bessel functions, we can 


\section{Problematic Spheroids}

deduce that

$$
F_{n k}^{+}=-s^{k+1} \sum_{q=q_{\min }}^{\infty} \gamma_{q n k} \frac{(-1)^{q}}{2^{q} q !} x^{2 q+k-n+2}
$$

where

$$
\begin{aligned}
\gamma_{q n k} & =\sum_{i=0}^{q} c_{i q n k} \\
c_{i q n k} & =\left(\begin{array}{c}
q \\
i
\end{array}\right) a_{i, n} b_{q-i, k} s^{2(q-i)}
\end{aligned}
$$

$\left(\begin{array}{l}q \\ i\end{array}\right)$ is the binomial coefficient $\frac{q !}{i !(q-i) !}$, and $a_{i, n}$ and $b_{q-i, k}$ are as defined in Appendix A. These expressions are a formally correct method to calculate the desired positive part of our product, but they suffer some numerical problems. A demonstration of this is provided in Fig. 5.5. We can see that for $s$ close to 1, as well as for larger values of $n-k$ (that is, further from the diagonal), there is a loss of precision. As well, there is a loss of precision for small $n$ values when $x$ is large.

In order to work around some of these numerical issues, the first approach is to modify how the coefficients are calculated. The problem which is worse for $s$ close to one may be treated by re-writing the form of the coefficients. Instead of $s^{2(q-i)}$ in Eq. 5.91, for the case where $q \leq n-k-1$, we instead write

$$
s^{2(q-i)}=\left(s^{2}-1+1\right)^{q-i}=\sum_{j=0}^{q-i}\left(\begin{array}{c}
q-i \\
j
\end{array}\right)\left(s^{2}-1\right)^{j}
$$

and thus $\gamma_{q n k}$ may be written

$$
\gamma_{q n k}=\sum_{i=0}^{q}\left(\begin{array}{l}
q \\
i
\end{array}\right) a_{i, n} b_{q-i, k} \sum_{j=0}^{q-i}\left(\begin{array}{c}
q-i \\
j
\end{array}\right)\left(s^{2}-1\right)^{j} .
$$




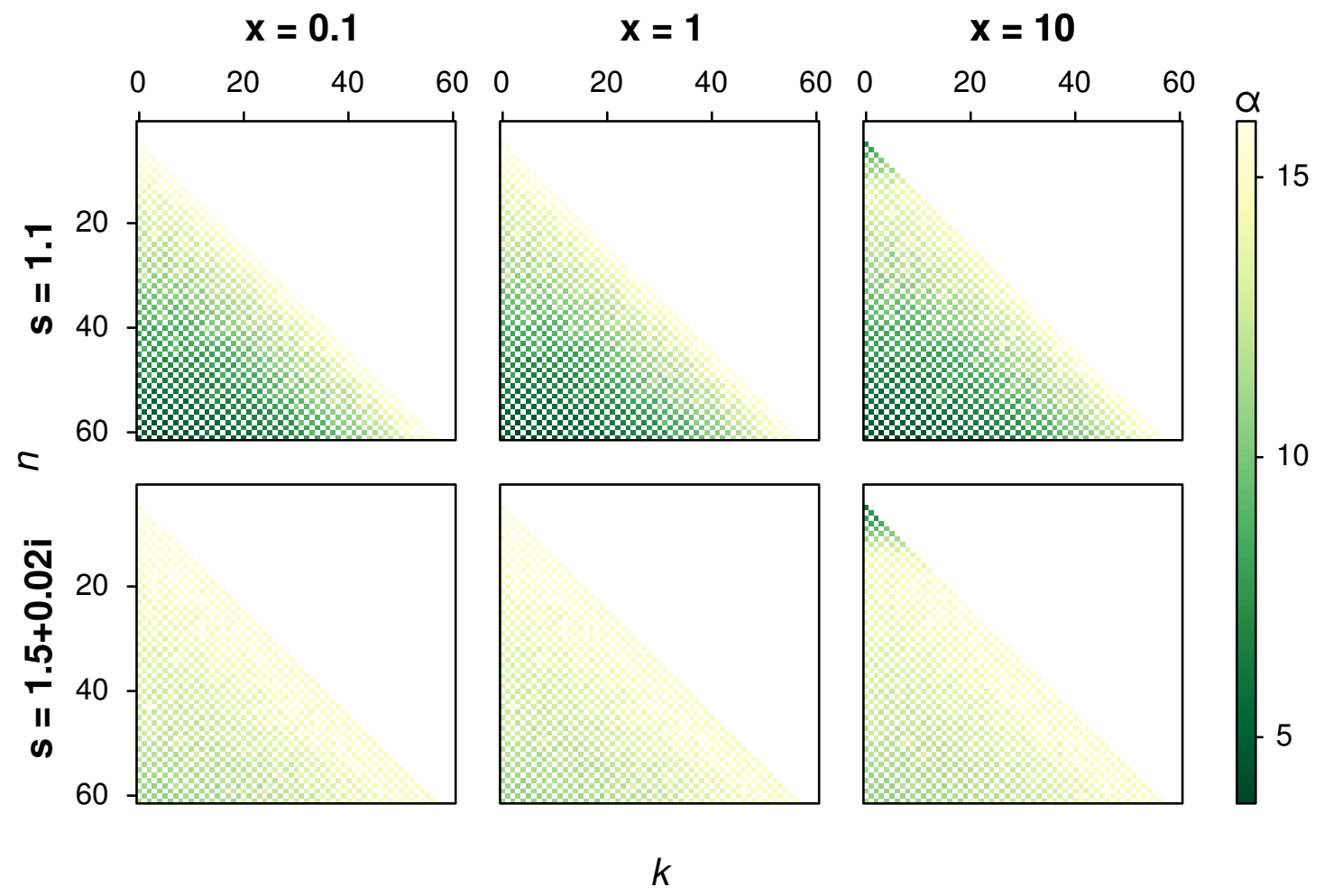

Figure 5.5: A demonstration of the numerical problems with the naïve series implementation for $\mathrm{F}^{+}$. This shows the number of correct digits, when compared to an arbitrary-precision implementation. This shows only those entries with $n+k$ even in the region where cancellations are present. In other regions (above the diagonal), $F_{n k}^{+}=F_{n k}$, and this method is not required. 


\section{Problematic Spheroids}

By swapping the order of the double sum, as

$$
\sum_{i=0}^{q} \sum_{j=0}^{q-i}=\sum_{j=0}^{q} \sum_{i=0}^{q-j}
$$

we may write

$$
\gamma_{q n k}=\sum_{j=0}^{q}\left(\begin{array}{l}
q \\
j
\end{array}\right)\left(s^{2}-1\right)^{j} w_{q-j}
$$

where

$$
w_{r}=\sum_{i=0}^{r}(-1)^{i}\left(\begin{array}{l}
r \\
i
\end{array}\right) \frac{(2 n-2 i-1) ! !}{(2 k+2 q-2 i+1) ! !}
$$

where we have used the forms of $a_{i, n}$ and $b_{q-i, k}$ for $i \leq n$.

The term $(2 n-2 i-1) ! ! /(2 k+2 q-2 i+1) !$ ! is a polynomial in $i$ of degree $n-k-q-1$, and using the relation for a general polynomial $P$ of degree less than $q$,

$$
\sum_{i=0}^{q}(-1)^{i}\left(\begin{array}{l}
q \\
i
\end{array}\right) P(i)=0
$$

then $w_{r}=0$ for $n-k-q-1<1$, so the sum in $\gamma_{q n k}$ only needs to consider $j$ for $j \geq \max \left(0,2\left(q-q_{\min }\right)-1\right)$. In order to calculate the other values of $w_{r}$, we write (with implicit $n$-dependence)

$$
\begin{aligned}
u_{r b} & =\left.2^{b}\left(\frac{\mathrm{d}}{\mathrm{d} X}\right)^{b}\left[X^{n-1 / 2}\left(1-\frac{1}{X}\right)^{r}\right]\right|_{X=1} \\
& =\left.2^{b}\left(\frac{\mathrm{d}}{\mathrm{d} X}\right)^{b}\left[\sum_{i=0}^{r}(-1)^{i}\left(\begin{array}{c}
r \\
i
\end{array}\right) X^{n-i-1 / 2}\right]\right|_{X=1}
\end{aligned}
$$

By performing the successive differentiations, we can see that in the case of 
$0 \leq b \leq n-r-1$

$$
u_{r b}=\sum_{i=0}^{r}(-1)^{i}\left(\begin{array}{l}
r \\
i
\end{array}\right) \frac{(2 n-2 i-1) ! !}{(2 n-2 i-2 b-1) ! !}
$$

and hence

$$
w_{r}=u_{r, n-k-q-1}
$$

and so

$$
\gamma_{q n k}=\sum_{j=\max \left(0,2\left(q-q_{\min }\right)-1\right)}^{q} \beta_{j q} u_{q-j, n-k-q-1}
$$

where

$$
\beta_{j q}=\left(\begin{array}{l}
q \\
j
\end{array}\right)\left(s^{2}-1\right)^{j}
$$

These values $\beta$ and $u$ are both independent of $k$, and hence for efficiency may be calculated for each $n$ and then used for multiple $k$ values. As we shall see, this matches closely the preferred way of calculating all of the values. For numerical stability, $\beta$ and $u$ are calculated via recursion. For $\beta$, we have

$$
\begin{aligned}
\beta_{0, q} & =1, \\
\frac{\beta_{j, q}}{\beta_{j-1, q}} & =\frac{q-j+1}{j}\left(s^{2}-1\right)
\end{aligned}
$$

while for $u_{r b}$, we may derive the relations

$$
\begin{aligned}
u_{0,0} & =1 \\
u_{r, 0} & =0 \quad \text { for } r>0 \\
u_{r, b+1} & =\left(n-\frac{1}{2}-2 r\right) u_{r b}-\left(n-\frac{1}{2}-r\right) u_{r+1, b}
\end{aligned}
$$




\section{Problematic Spheroids}

and use these to calculate $u_{r b}$.

In the other case, where $q \geq n-k$, there is not such a severe numerical problem that needs to be overcome, but nonetheless for efficiency we design a recursion scheme to calculate $c_{i q n k}$. This applies when $q \geq n-k$. The relation is (indices $n$, $k$, implied)

$$
\frac{c_{i, q}}{c_{i+1, q}}=\frac{s^{2}(i+1)(2 i+1-2 n)}{(q-i)(2 k+2 q-2 i+1)}
$$

which we use with a downward recursion scheme. The initial values, for $i=q$, are obtained with the upwards recursion

$$
\frac{c_{q+1, q+1}}{c_{q, q}}=\frac{1}{2 q+1-2 n}
$$

and we need to know the values for $i=q=n-k$, which are simply

$$
c_{n-k, n-k, n, k}=a_{n-k, n} b_{0, k}=\frac{1}{2 k+1} .
$$

Thus we are able to calculate $\gamma_{q n k}$ for all values of $q$. We then write

$$
F_{n k}^{+}(x)=-s^{k+1} \sum_{q=q_{\min }}^{\infty} \gamma_{q n k} \alpha_{q n k}(x)
$$

where

$$
\alpha_{q n k}(x)=\frac{(-1)^{q}}{2^{q} q !} x^{2 q+k-n+2} .
$$

For numerical efficiency, we calculate $\alpha_{q n k}(x)$ as

$$
\frac{\alpha_{q, n, k}(x)}{\alpha_{q-1, n, k}(x)}=\frac{-x^{2}}{2 q}
$$




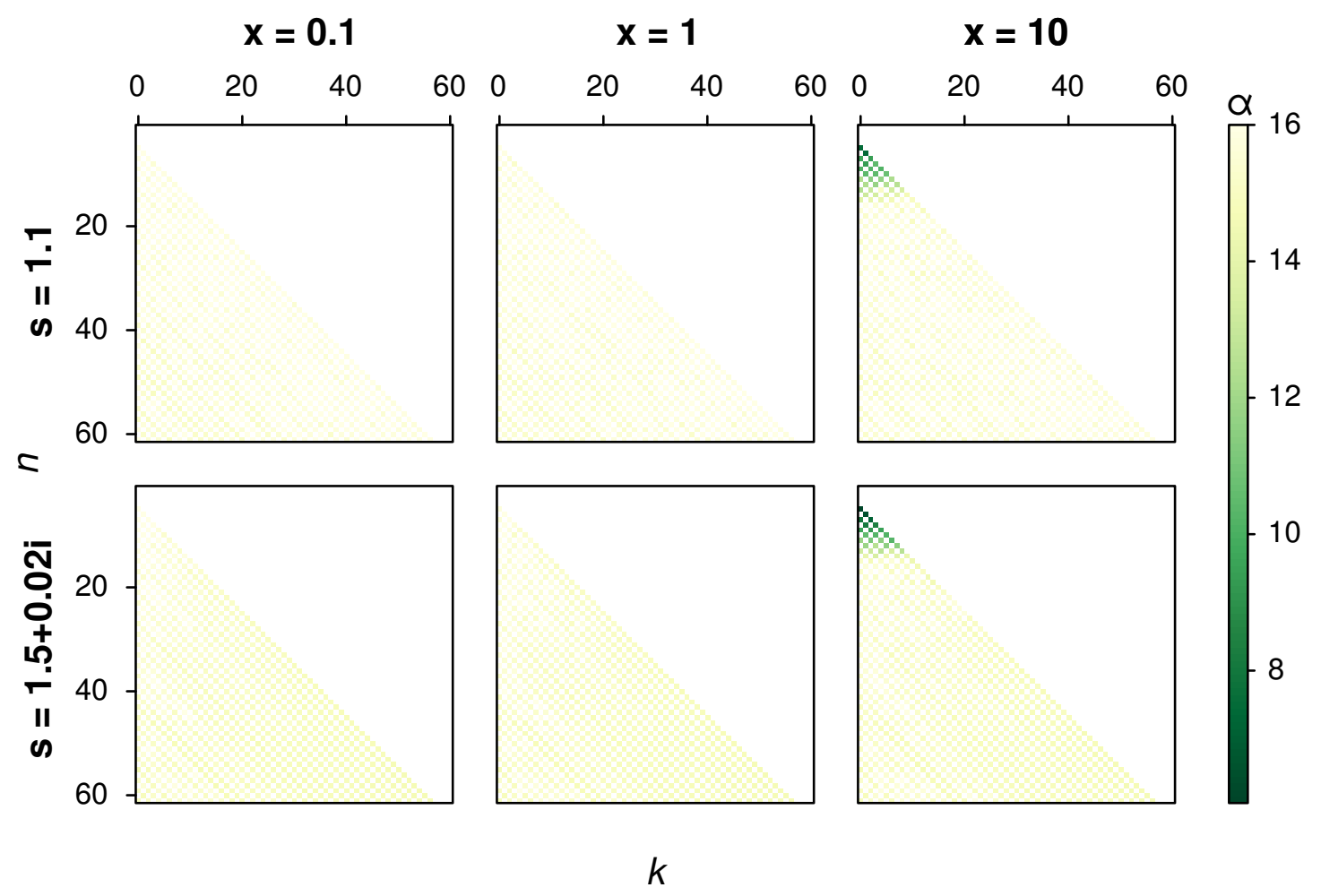

Figure 5.6: A demonstration of the numerical performance with the improved series implementation for $F^{+}$. This shows the number of correct digits, when compared to an arbitrary-precision implementation. The loss of precision for low $n, k$ values for large sizes is not problematic in reality, using methods discussed in the next section. This shows only those entries with $n+k$ even in the region where cancellations are present. In other regions (above the diagonal), $F_{n k}^{+}=F_{n k}$, and this method is not required. 


\section{Problematic Spheroids}

and we are able to calculate $\bar{\alpha}_{n k}=\alpha_{q_{\min }, n, k}(x)$ by recursing on $k$ as

$$
\begin{aligned}
\bar{\alpha}_{n, k=n-1}(x) & =1 \\
\frac{\bar{\alpha}_{n k}(x)}{\bar{\alpha}_{n, k+2}(x)} & =\frac{-1}{n-k-2} .
\end{aligned}
$$

The sum is calculated by adding on terms, until three consecutive terms have made no contribution (within double precision). However, when $q=n-k$ the form of $\gamma_{q n k}$ changes between our two expressions, and we must consider the possibility that the new terms start to make a significant contribution. As a result, we ensure that even if subsequent terms with $q<n-k$ have stopped making contributions, terms from $q=n-k$ are considered, until three consecutive of those terms also make no contribution. This step is most significant when $s$ is close to one, when the terms for $q<n-k$ typically become small.

Our implementation removes the need for special consideration when $s$ is near one. However, for completeness, a demonstration of the cause of such potential problems is provided in Appendix $\mathrm{F}$.

We are now able to examine the numerical performance of this series implementation of generating $F^{+}$. We can see in Fig. 5.6 that the performance compared to the naïve implementation (Fig. 5.5) is now much improved, with only some loss of precision for low- $n$ for large sizes. This turns out not to be important in real applications, and a method to work around this is presented in the next section.

\subsubsection{Recursion to Fill the Matrix}

The series method to calculate the values of $F_{n k}^{+}$works well for entries with large $n$, while for large sizes, it performs badly for entries with low $n$. In order to calculate these problematic entries with higher precision, we use a different approach. For this, we calculate entries where the series approach works well, and then use recursion relations on the Bessel functions to calculate the values where the series works badly. This has the benefit that the series, which is time-consuming, only needs to be calculated for a subset of the required values, and then a fast recursion scheme 
may be used to populate the rest of the matrix.

When designing the recursion scheme, it must be based on the recursion relations of the Bessel functions. These are

$$
\chi_{n-1}(x)+\chi_{n+1}(x)=\frac{2 n+1}{x} \chi_{n}(x)
$$

and similarly for $\psi_{k}(s x)$. From the former, we may obtain

$$
F_{n+1, k}=\frac{2 n+1}{x} F_{n k}-F_{n-1, k}
$$

and by considering the Taylor expansions of the series this may be written in terms of $F^{+}$as

$$
F_{n+1, k}^{+}(x)=\frac{2 n+1}{x}\left(F_{n k}^{+}(x)-F_{n k}^{+}(0)\right)-F_{n-1, k}^{+}(x) .
$$

However, implementing this leads to a loss of precision, as $F_{n k}^{+}(x)$ and $F_{n k}^{+}(0)$ may be close in value to each other. In order to obtain a relation without this difference, we combine the recursion relations for $\chi_{n}(x)$ (given in Eq. (5.115) ) and $\psi_{k}(s x)$, which is

$$
\psi_{k+1}(s x)+\psi_{k-1}(s x)=\frac{2 k+1}{s x} \psi_{k}(s x)
$$

to obtain the relation (also valid on $F_{n k}$, without the ${ }^{+}$)

$$
F_{n+1, k}^{+}+F_{n-1, k}^{+}=s \frac{2 n+1}{2 k+1}\left(F_{n, k+1}^{+}+F_{n, k-1}^{+}\right) .
$$

From Eq. (5.119), there are four possible schemes to populate the matrix. Each of these requires a different set of starting values, and has different numerical performance. These schemes are summarised in Fig. 5.7. The 'natural' method in some sense is shown in Fig. 5.7(d). This seems natural as the required starting values are $k=1$, and the diagonal, so if a larger number $N$ is required, previous starting values may be reused. However, this scheme has poor numerical performance, with the 


\section{Problematic Spheroids}

error becoming large as we move away from the diagonal. It is possible to examine the error in two ways. Knowing the exact result from arbitrary-precision code, the effect of each step may be calculated, using the correct value as the starting values in each case, or alternatively (as shown here) the error accumulated across many steps, using the correct values only for the initial starting value may be obtained. The error behaves in a similar fashion for both of these approaches.

The schemes shown in Fig. 5.7(a) and (b) are not as appealing from the perspective of starting values, but also display a loss of precision. The optimal scheme is that shown in Fig. 5.7(c), which has excellent precision behaviour. The starting values are manageable, as our series method (as presented in Section 5.6.2) is able to efficiently generate an entire row of the matrix (that is, for one value of $n$, calculate all problematic values of $k$ ). It would be nice to be able to easily increase the value of $N$ without redoing the entire calculation, but the calculations do not take so long that this is a problem, and the vastly superior performance with regards to precision easily wins that battle. As a result, we solve for $F_{n, k-1}^{+}$, using the relation

$$
F_{n, k-1}^{+}=\frac{2 k+1}{s(2 n+1)}\left(F_{n+1, k}^{+}+F_{n-1, k}^{+}\right)-F_{n, k+1}^{+}
$$

Thus, by using this equation, we are able to easily generate all of the entries for $F^{+}$that we require. It is necessary to pick a starting $N$ (for which the series implementation must provide accurate results), and then the rest of the matrix may be populated. For cases when only a small $N$ is required, if the series method fails to provide accurate results for that $N$, it is necessary to use a larger $N$ for the series, and then the final matrix may be truncated if desired. We may use the values calculated for $F^{+}$to calculate all modified Bessel products that we require, as discussed in the next section.

\subsubsection{Derivatives}

Once we have calculated $F^{+}$, it is necessary to calculate the quantities that are required for the integrals. These required values are $\left(x \chi_{n} \psi_{k}\right)^{+},\left(x \chi_{n}^{\prime} \psi_{k}\right)^{+},\left(x \chi_{n} \psi_{k}^{\prime}\right)^{+}$, $\left(x \frac{\chi_{n} \psi_{k}}{s x^{2}}\right)^{+},\left(x \chi_{n}^{\prime} \psi_{k}^{\prime}+n(n+1) x \frac{\chi_{n} \psi_{k}}{s x^{2}}\right)^{+}$and $\left(x \chi_{n}^{\prime} \psi_{k}^{\prime}+k(k+1) x \frac{\chi_{n} \psi_{k}}{s x^{2}}\right)^{+}$. The first of 


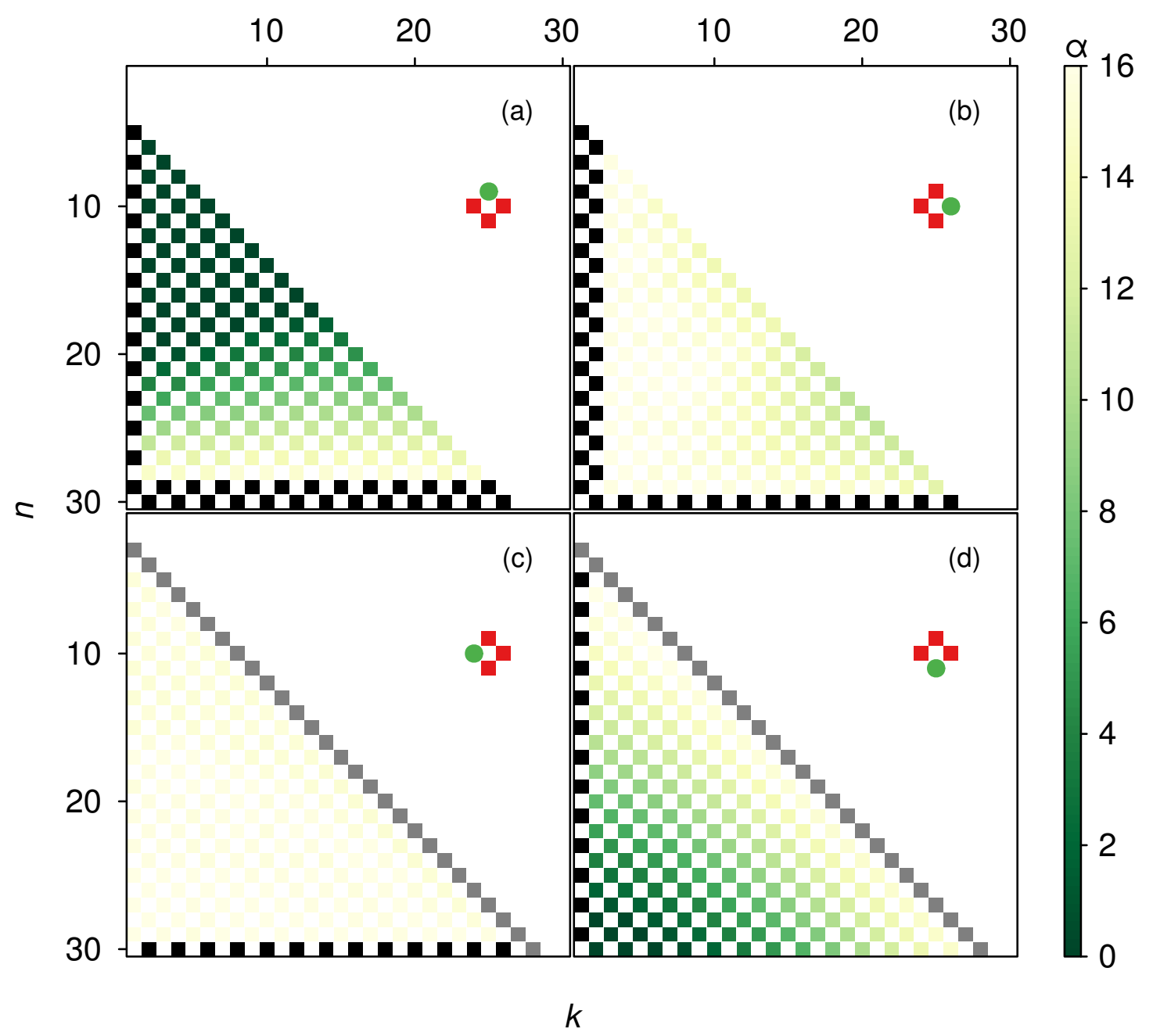

Figure 5.7: A demonstration of the performance of the different recursion schemes to populate $F^{+}$. This shows the number of correct digits, when compared the arbitrary-precision series implementation. Black entries represent entries that are necessary to start the recursion, but which have cancellations, and so must be calculated with the series implementation. Grey entries are entries with no cancellation that are necessary to start the recursion. These may be calculated simply as the product of the appropriate Bessel functions. The inset figures denote how the recursion scheme works, with the entry shown as a green circle being calculated by some combination of the entries shown as red squares. The parameters used here were $x=0.1$ and $s=1.5+0.02 i$. 


\section{Problematic Spheroids}

these is easy to generate, as it is simply $F^{+}$. There are relations for the Bessel functions,

$$
\begin{aligned}
(2 n+1) \chi_{n}^{\prime}(x) & =(n+1) \chi_{n-1}(x)-n \chi_{n+1}(x) \\
(2 k+1) \psi_{k}^{\prime}(s x) & =(k+1) \psi_{k-1}(s x)-k \psi_{k+1}(s x)
\end{aligned}
$$

from which we may obtain the relations

$$
\begin{aligned}
\left(x \chi_{n} \psi_{k}^{\prime}\right)^{+} & =\frac{(k+1) F_{n, k-1}^{+}-k F_{n, k+1}^{+}}{2 k+1} \\
\left(x \chi_{n}^{\prime} \psi_{k}\right)^{+} & =\frac{(n+1) F_{n-1, k}^{+}-n F_{n+1, k}^{+}}{2 n+1}
\end{aligned}
$$

as well as

$$
\begin{aligned}
\left(x \chi_{n}^{\prime} \psi_{k}^{\prime}+n(n+1) x \frac{\chi_{n} \psi_{k}}{s x^{2}}\right)^{+}= & \frac{1}{(2 n+1)(2 k+1)} \\
& \times\left\{(n+k+1)\left[(n+1) F_{n-1, k-1}^{+}+n F_{n+1, k+1}^{+}\right]\right. \\
& \left.+(n-k)\left[(n+1) F_{n-1, k+1}^{+}+n F_{n+1, k-1}^{=}\right]\right\} \\
\left(x \chi_{n}^{\prime} \psi_{k}^{\prime}+k(k+1) x \frac{\chi_{n} \psi_{k}}{s x^{2}}\right)^{+}= & \frac{1}{(2 n+1)(2 k+1)} \\
& \times\left\{(n+k+1)\left[(k+1) F_{n-1, k-1}^{+}+k F_{n+1, k+1}^{+}\right] .\right. \\
& \left.+(k-n)\left[(k+1) F_{n+1, k-1}^{+}+k F_{n-1, k+1}^{=}\right]\right\}
\end{aligned}
$$

These relations allow all of the required integrands to be calculated. It is worth mentioning that it is because these relations depend on entries $n-1, n+1, k-1$, $k+1$ that the Bessel functions must be calculated for $n, k$ from 0 to $N+1$. Thus, after applying these relations, all of the integrands are available for $n, k$ from 1 to $N$.

It is worth commenting on the new form of the integrals, as we use terms like 
$F_{n k}^{+}(s, x) / x$ in them. The integrals for $\mathbf{U}$ (and not $\mathbf{P}$ ) are

$$
\begin{aligned}
K_{n k}^{1} & =\int_{0}^{\pi} \mathrm{d} \theta \sin \theta \pi_{n} d_{k} \frac{x_{\theta}}{x}\left[x \chi_{n} \psi_{k}^{\prime}\right]^{+} \\
K_{n k}^{2} & =\int_{0}^{\pi} \mathrm{d} \theta \sin \theta \pi_{n} d_{k} \frac{x_{\theta}}{x}\left[x \chi_{n}^{\prime} \psi_{k}\right]^{+} \\
L_{n k}^{5} & =\int_{0}^{\pi} \mathrm{d} \theta \sin \theta\left[n(n+1) d_{n} \tau_{k}-k(k+1) \tau_{n} d_{k}\right] \frac{x_{\theta}}{x} F_{n k}^{+}(s, x) \\
L_{n k}^{7} & =\int_{0}^{\pi} \mathrm{d} \theta \sin \theta \tau_{n} d_{k} \frac{x_{\theta}}{x}\left[x\left(\chi_{n}^{\prime} \psi_{k}^{\prime}+n(n+1) \frac{\chi_{n} \psi_{k}}{s x^{2}}\right)\right]^{+} \\
L_{n k}^{8} & =\int_{0}^{\pi} \mathrm{d} \theta \sin \theta d_{n} \tau_{k} \frac{x_{\theta}}{x}\left[x\left(\chi_{n}^{\prime} \psi_{k}^{\prime}+k(k+1) \frac{\chi_{n} \psi_{k}}{s x^{2}}\right)\right]^{+} .
\end{aligned}
$$

The forms for the diagonal entries are unchanged, as those entries do not have cancellations. As well, $\mathbf{P}$ does not have cancellations in the integrals, so the expressions are not altered. There is, however, a minor cancellation in the calculation of the integrand on the diagonal of $\mathbf{P}^{11}$, which is described in the next section.

\subsubsection{A Cancellation in $P$}

While so far all of the cancellations have been in $\mathbf{U}$, there is one cancellation that we have noticed in $\mathbf{P}$. This results in a loss of precision for the diagonal entries of $\mathbf{P}^{11}$ in the case of small size parameters. This loss of precision is evident in the calculation

$$
\psi_{n}^{\prime}(x) \psi_{n}(s x)-s \psi_{n}(x) \psi_{n}^{\prime}(s x)
$$

in the calculation of $\tilde{L}_{n}^{1}$ (which is only for the diagonal elements). The loss of precision is in the calculation of the integrand, not in the integration itself. It comes about as a cancellation in the leading order terms (which are of order $x^{2 n+1}$ ), while the integral should scale as $x^{2 n+3}$. We may use another of the relations on the Bessel 


\section{Problematic Spheroids}

functions,

$$
\begin{gathered}
\psi_{n}^{\prime}(x)=-\psi_{n+1}(x)+\frac{n+1}{x} \psi_{n}(x) \\
\psi_{n}^{\prime}(s x)=-\psi_{n+1}(s x)+\frac{n+1}{s x} \psi_{n}(s x)
\end{gathered}
$$

to write

$$
\psi_{n}^{\prime}(x) \psi_{n}(s x)-s \psi_{n}(x) \psi_{n}^{\prime}(s x)=s \psi_{n}(x) \psi_{n+1}(s x)-\psi_{n+1}(x) \psi_{n}(s x)
$$

Both of the new terms scale as $x^{2 n+3}$, and no longer exhibit a loss of precision. This slightly complicates the calculation of the entries, as it mixes the orders of the Bessel functions.

\subsubsection{Demonstration of Improved Performance}

Now that we have new expressions for the integrals, designed to avoid problems with numerical loss of precision, it is important to check that the results from these expressions are correct. A comparison with arbitrary-precision results using the previous expressions is shown in Fig. 5.8, while for the new expressions a comparison is given in Fig. 5.9. Here we can see that the extreme loss of precision with the previous expressions is no longer evident with the new expressions, which have a high level of agreement. This confirms that the terms that we have removed from the integrals were the cause of the loss of precision, and the new expressions are correct.

Now that we have reliable $P$ - and $Q$-matrices, it is possible to test the precision of the $T$-matrix after inversion. In comparision with Fig. 5.4 (which made use of the old double-precision code), we can see in Fig. 5.10 that the new double-precision code is able to calculate $\mathbf{T}$ to a high level of agreement with arbitrary-precision inversion. We are also able to use the new codes to test how different elements of $\mathbf{T}$ converge as we increase the size of $\mathbf{P}$ and $\mathbf{Q}$. Studying this is a substantial topic, but some preliminary results are presented here. The basic idea is to test the effect of matrix truncation, by increasing the size of $\mathbf{P}$ and $\mathbf{Q}$, and testing to what 

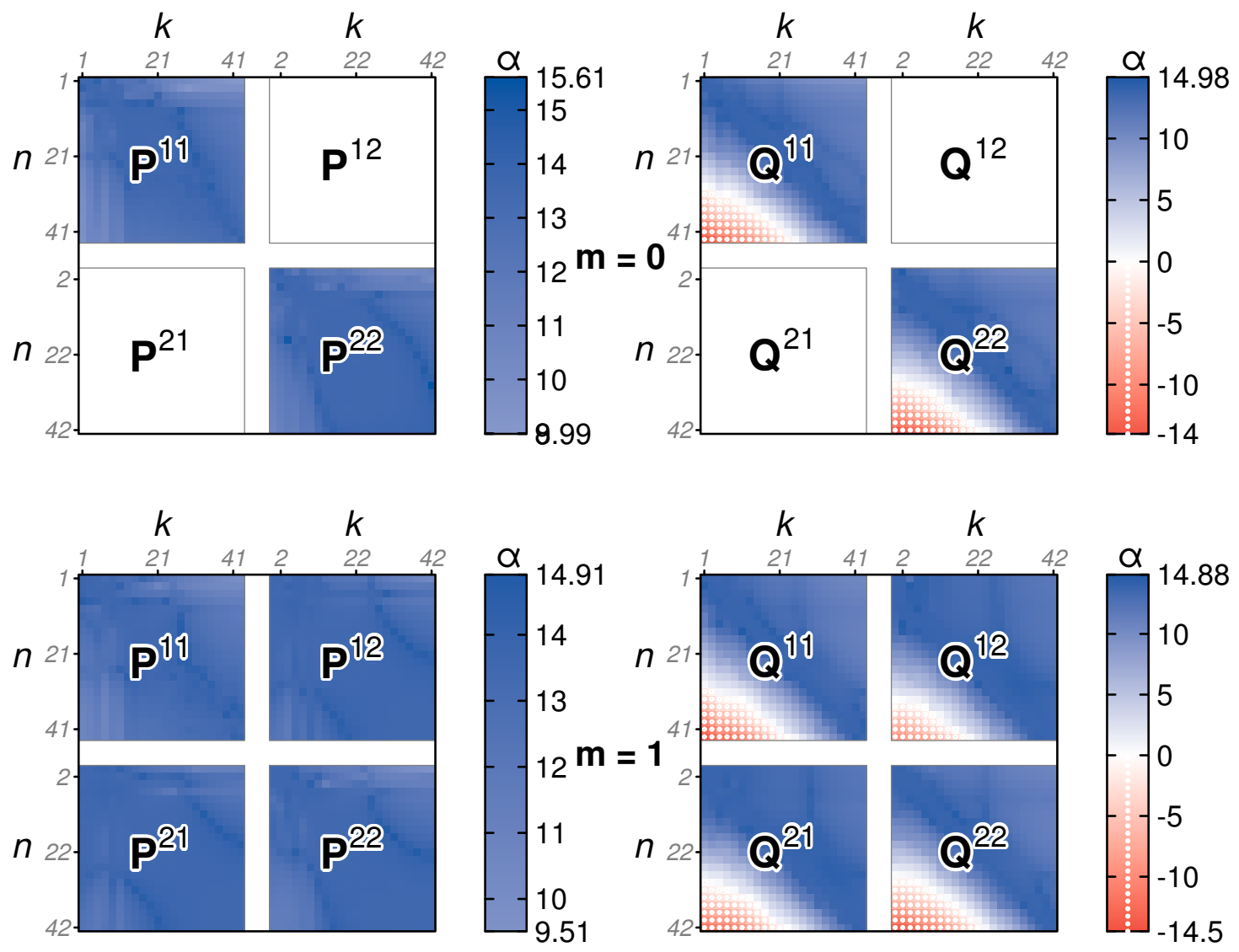

Figure 5.8: This shows the agreement with the arbitrary precision code of the $\mathbf{P}$ and Q matrices, when calculated using the 'old' expressions, which do not try and remove the cancellations. This is for a prolate spheroid of relative refractive index $s=1.5+02 i$, size parameter $x=10$, and aspect ratio $h=2$. Both cases $m=0$ (top row) and $m=1$ (bottom row) are shown. Entries in red, with inset dots, have incorrect orders of magnitude. Only one parity of each $n$ and $k$ is shown in each panel, as indicated by the axis labels. The off-diagonal blocks for $m=0$ are empty, as the integrals are identically zero in those cases. 

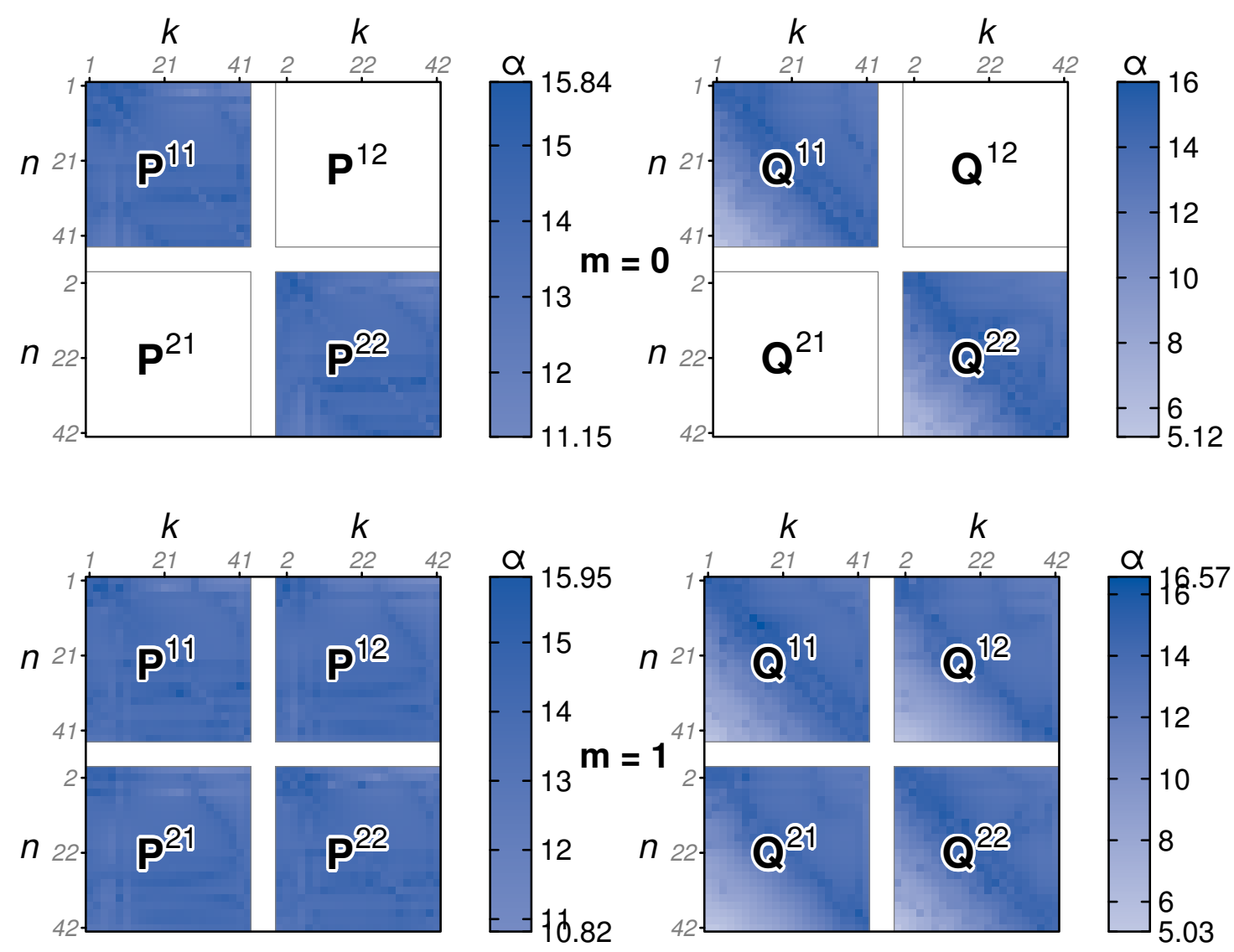

Figure 5.9: This shows the agreement between results with arbitrary precision code and double precision code using the new expressions. This is for a prolate spheroid of relative refractive index $s=1.5+02 i$, size parameter $x=10$, and aspect ratio $h=2$. Both cases $m=0$ (top row) and $m=1$ (bottom row) are shown. Only one parity of each $n$ and $k$ is shown in each panel, as indicated by the axis labels. The off-diagonal blocks for $m=0$ are empty, as the integrals are identically zero in those cases. 
order all the values in $\mathbf{T}$ agree to some defined precision $\alpha$. Results are shown in Fig. 5.11. This shows that in order for the $T_{11}^{11}$ and $T_{11}^{22}$ entries to be correct, the $P$ - and $Q$-matrices must have $N=15(\alpha=4)$ or $N=30(\alpha=12)$. However, once this value is correct, then roughly one extra $N$ is correct in $\mathbf{T}$ for each additional $N$ in $\mathbf{P}, \mathbf{Q}$. This means that the size of $\mathbf{P}, \mathbf{Q}, N_{Q}$, should be chosen to be larger than the number of terms desired in $\mathbf{T}$, such as $N_{Q}=N+\Delta$. The value of $\Delta$ will depend on the required precision of $\mathbf{T}$, as well as dependence on the size and aspect ratio of the particle being considered. The exact dependence on these quantities is an area of further study.

\section{Time Requirements}

It is expected that this new method of calculating the $P$ - and $Q$-matrices will not have a significant performance penalty, as the modified code is independent of the index $m$. That is, for each calculation, the Bessel product is calculated once, and for each $m$, the angular functions are calculated, and the integration performed. As a result of the modified code not having $m$-dependence, the performance of it is not greatly impacted.

In order to demonstrate the time requirements, the time taken to calculate $\mathbf{P}$ and $\mathbf{Q}$ for this new method, unmodified MATLAB code (whose results might contain severe numerical errors), and converged arbitrary-precision results are presented in Fig. 5.12. The computer used for these measurements is a quad-core Intel Xeon W3520 $2.67 \mathrm{GHz}$ desktop with $18 \mathrm{~GB}$ of RAM, running MATLAB R2012a and Ubuntu Linux 13.04. The particle modelled here is a prolate spheroid, with size parameter $x_{\max }=2$, aspect ratio $h=2$, relative refractive index $s=1.5+0.02 i$, and $k_{1}=1$, ranging from $N=5$ to $N=50$. Here we can see that the arbitraryprecision code represents a significant performance penalty, while the new code is much faster. The disadvantage of the old code is that the results begin to fail catastrophically for $N$ larger than approximately 15 . 

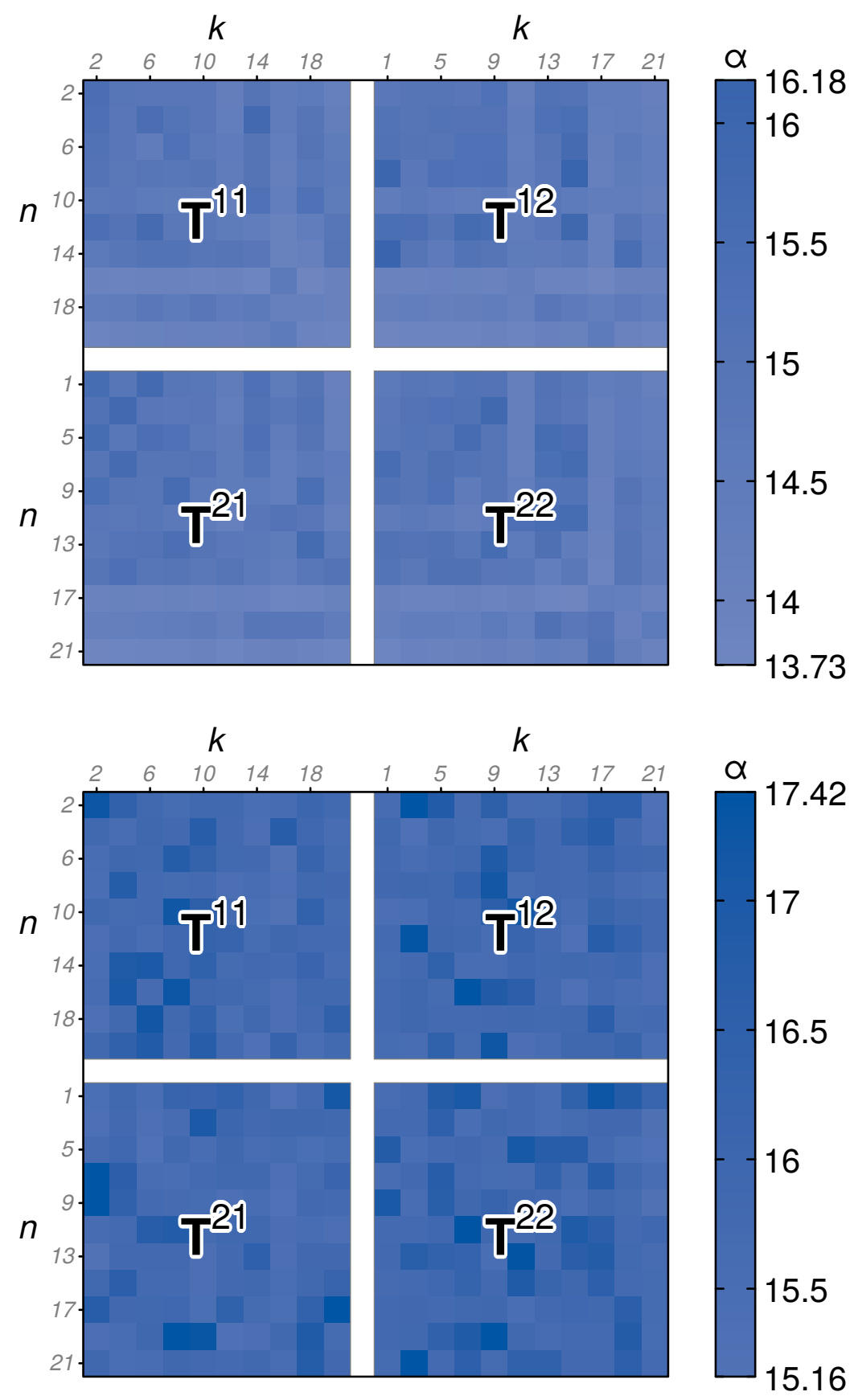

Figure 5.10: Number of correct digits of the $T$-matrix after inversion in double precision, after $\mathbf{P}$ and $\mathbf{Q}$ were calculated in (top) double precision using our new method and (bottom) arbitrary precision. This is relative to inversion also being carried out in arbitrary precision. The particle here is a prolate spheroid of aspect ratio $h=2$, size parameter $x_{\max }=1$, with relative refractive index $s=1.5+0.02 i$. Only $m=1$ is considered here. This shows only one parity of each $n, k$, for each block of the matrix, as indicated by the axis labels, though the other entries behave similarly. 


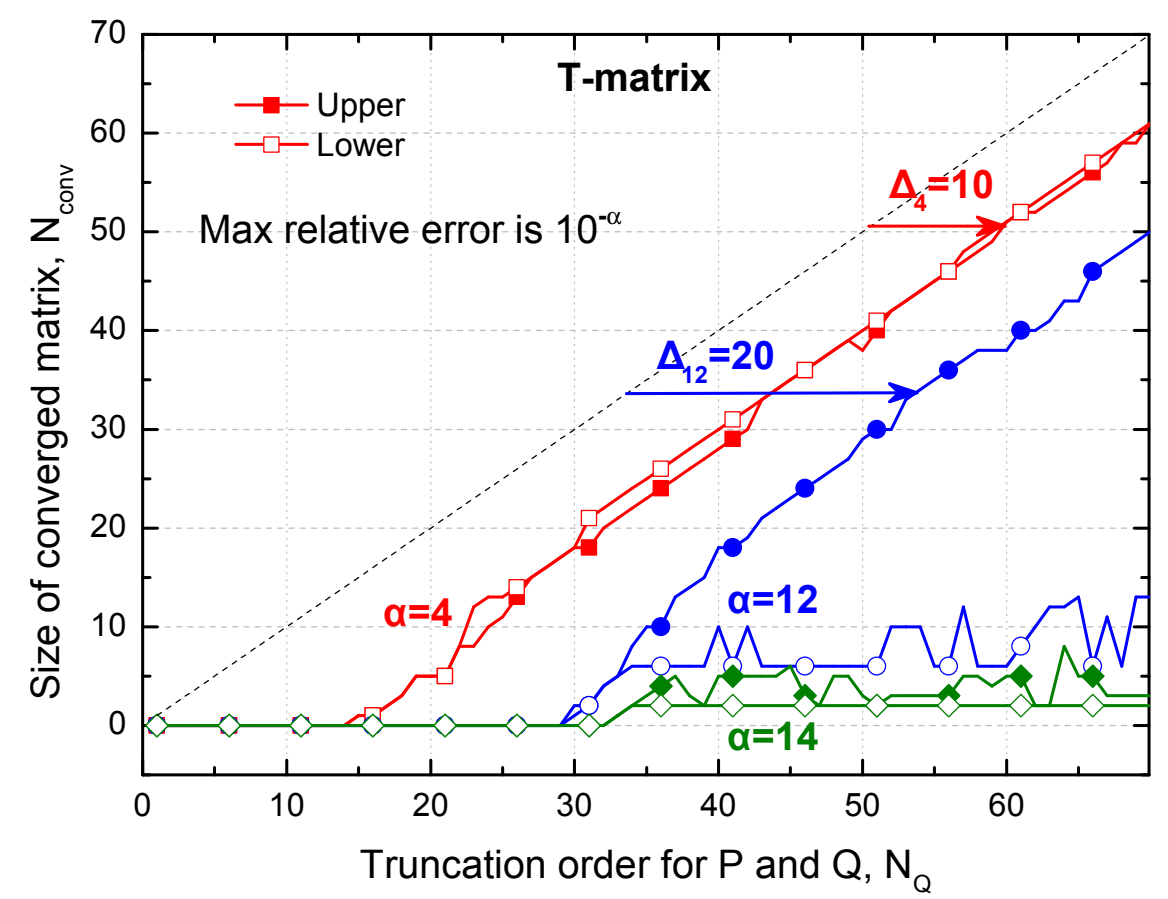

Figure 5.11: The size of the converged sub-matrix of $\mathbf{T}$ as the size of $\mathbf{P}$ and $\mathbf{Q}$ is varied, for different values of required relative precision $\alpha$. The maximum order for the upper- and lower-triangular parts of $\mathbf{T}$ are shown, but by symmetry the lower triangle may be obtained from the upper triangle. 


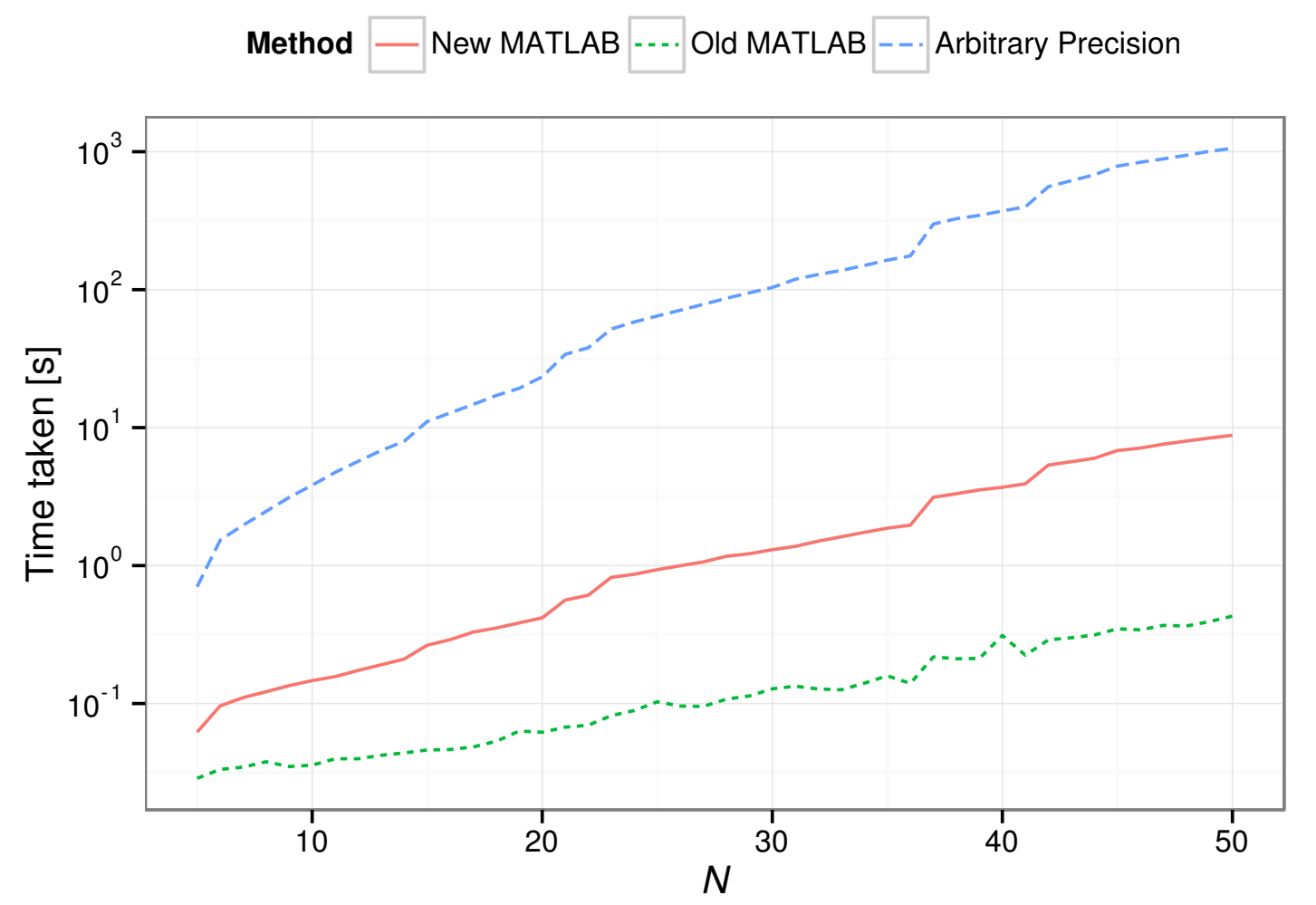

Figure 5.12: Measured times taken to compute $\mathbf{P}$ and $\mathbf{Q}$ using three possible EBCM implementations, for a prolate spheroid. The three different methods are an arbitrary-precision C implementation, a naïve (old) MATLAB code that does not treat spheroids specially, and a new MATLAB code implemented using the method presented here, which removes cancellations for spheroids. The precision used for the arbitrary-precision code varies in order that the final results are correct to double precision, and the number of integration points likewise. These values range from 73 to 300 bits for the precision (for the arbitrary-precision code), and 80 to 500 for the number of integration points. All methods used the same number of integration points, and calculate for all values of $m$ from 0 to $N$. 


\subsection{Discussion and Outlook}

\subsection{Discussion and Outlook}

The results in this chapter have provided an understanding of the main numerical problems that affect the EBCM calculations of spheroids (and offset spheres). The cause of these problems was problematic terms in the integrands, which should identically integrate to zero, but due to loss of precision, they dominate the integration and lead to dramatically incorrect results due to catastrophic cancellations. This specific numerical problem was found to be only problematic for some shapes, and it was shown that the dominant terms in the power series expansion of the integrand for some matrix entries of the $Q$-matrix do integrate identically to zero.

With knowledge of the cause of the numerical problems, focus turned to resolving these cancellations. This involved calculating the integrands without the terms in the power series that cause problems, which comes down to the calculation of the product of two Bessel functions, without the problematic terms. The best method that was found consists of using a series expansion to determine some values where the series expansion provides reliable results, and then using a recursion scheme to determine the other required matrix entries. Using this approach, it is now possible to obtain to a high precision the $T$-matrix for spheroids.

A demonstration of the improved performance of the new codes can be seen in Fig. 5.10. This ability to calculate the $T$-matrix to such a precision represents a substantial improvement in the performance of the $T$-matrix method for the case of spheroids. In the case of general shapes, the problems with the $T$-matrix method have a different origin, and this approach is not applicable. To improve other shapes, an approach in line with previous works seems the most promising, to focus on improving the matrix inversion [63, 64, 79]. However, as the issues that affect general shapes are different, this problem is independent from the problems with spheroids.

Now that it is possible to obtain an accurate $T$-matrix for spheroids, there is the possibility of further investigations, which have not been completed to date. These include examining the convergence of the near-field, specifically to study the so-called Rayleigh hypothesis, and to examine the regions where this may not be valid. While previously it was challenging to study this for spheroids, using this 


\section{Problematic Spheroids}

new method it is now possible to calculate high-order multipole contributions, and so determine how the field is converging. Some preliminary exploration of this has already been carried out by Stanley Roache, a summer student.

An additional area of investigation is to look at how the elements of $T$ behave as $N$ increases. As the matrices involved are infinite, they must be truncated. The size must be large enough that the terms in $\mathbf{T}$ that we are interested in are not affected by this finite size. By using these accurate integral values, it is now possible to investigate this behaviour. Some preliminary analysis of this has already been carried out, as shown in Fig. 5.11, and is ongoing. This suggests that truncation of $\mathbf{T}$ after inversion is useful to remove some values that have not yet converged, leaving only entries that have converged, and hence are reliable values. This study is only possible due to having an efficient and reliable means of calculating $\mathbf{T}$.

One of the consequences of the results in this chapter is that it is clear that spheroids and offset spheres have numerical behaviour that is different to other shapes. That is, these shapes exhibit more challenging behaviour in the integrals, but once the correct integrals are obtained, they have easier inversion. As a result, caution should be used when selecting shapes to use to test EBCM implementations, realising that spheroids and offset spheres are not representative examples. For offset spheres this is particularly important, as they are an attractive shape to test with, because the correct results are obtainable with Mie theory, and subsequent translation of the results. 


\section{Application of Electromagnetic Calculations to Plasmonics}

Following on from the fundamental light-scattering focus of the previous chapters, this chapter presents work that uses light scattering to study systems of interest in the field of plasmonics. The first two sections here are concerned with work that was carried out in support of specific experimental projects [2, 5], modelling the response of experimentally-motivated systems. The last section concerns simulations carried out to examine how the electromagnetic enhancement factor (EF) is distributed in plasmonic systems [4]. It is presently a challenging task to experimentally measure EF distributions, though attempts have been reported [82].

This chapter does not exclusively make use of the $T$-matrix method, but largely uses COMSOL to solve the problems, as it is able to consider more general geometries. COMSOL solves problems using the finite-element method. Some use, however, is made of the $T$-matrix when examining the EF distributions, where its much greater speed and level of numerical accuracy is of benefit.

(Vegan) Chocolate Cake Preheat the oven to $180^{\circ} \mathrm{C}$. In a $22 \mathrm{~cm}$ cake tin, mix together well 1.25 cup flour, 1 cup sugar, 0.33 cup cocoa, 1 tsp baking soda, and 0.5 tsp salt. Add 1 cup warm water, 1 tsp vanilla essence, 0.33 cup vegetable oil, and 1 tsp white vinegar, and mix together well. Bake for $30 \mathrm{~min}$, or until a skewer comes out clean. When cool (on a rack), top with chocolate glaze. 


\subsection{Bipyramids and Bicones}

The basis of this section concerns bipyramids as used as a SERS substrate 2]. A transmission election microscopy (TEM) image of a bipyramid is presented in Fig. 6.1. In these single-molecule SERS (SM-SERS) experiments, the positivelycharged analytes (in this case, the dye Crystal Violet) are prevented from adsorbing on the surface of the bipyramids (except at the tips) by the similarly-charged layer of the surfactant, cetyltrimethylammonium bromide (CTAB). The CTAB layer is not present at the tips as the high curvature there disrupts the bilayer structure. This is a fortunate, if not entirely unrelated, coincidence, as the largest enhancement factor is also found at the tips. As a result, all of the adsorbed molecules end up in a hot-spot. In the case of SM-SERS, this allows for both an increased proportion and increased rate of single-molecule events 2 .

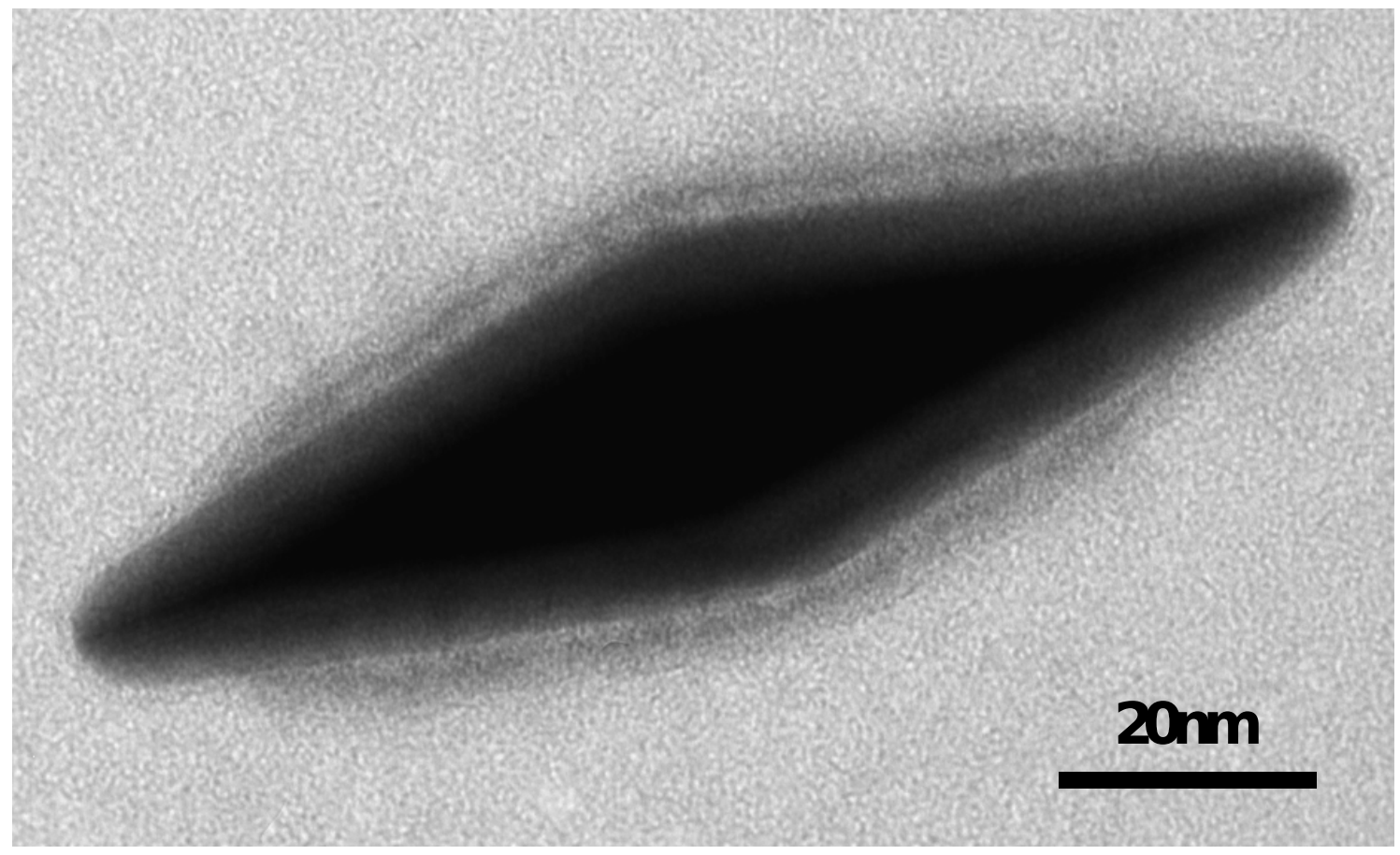

Figure 6.1: A TEM micrograph of a gold bipyramid. The bilayer of the surfactant CTAB is visible as the lighter region near the surface, which is notably absent at the tips. Note that this image is from a different batch of bipyramids to that used in the SERS measurements, and significantly has a different size. 


\subsection{Bipyramids and Bicones}

The contribution to this work that is detailed in this thesis involves numerical simulations undertaken in order to support calculations of the ratio of average to maximum enhancement of the particles. Such a ratio is used (as discussed later) to argue that all of the molecules experience a similar enhancement factor, which is an indication that they are all adsorbed at the hot-spots, and not randomly over the surface of the particle.

\subsubsection{Geometry}

In order to model the light-scattering behaviour of these bipyramids, it is necessary to represent their geometry somehow. For simplicity, we use an axisymmetric geometry, and hence we ignore the facets of the real particles, and label this new shape a bicone. As the tips of the particles are curved, and not sharp as for a cone, we must round the tip of the bicone.

The obvious first choice for the tip is a truncated spherical tip, placed so that the first derivative of the surface remains continuous. With this model, the parameters that we are able to vary are the sphere radius, the width of the bicone, and the height of the bicone. However, with this geometry, it is not possible to match the experimental resonance location while maintaining a shape resembling the TEM image.

Following a sphere, the next shape to be tried for the tip is a truncated spheroid. This model allows an extra parameter, replacing the radius of the sphere with the two axis lengths of the spheroid. After making this modification, the resonance in absorption matches the observed position well. The position of the spheroid is chosen such that the first derivative of the shape is continuous. A schematic of this shape is shown in Fig. 6.2

The geometry is described by

$$
r(\theta)= \begin{cases}-\frac{a z}{\left(r_{p}-a\right)|\cos \theta|-\frac{z \sin \theta}{r_{p}-a}} & \text { if } \beta<\theta<\pi-\beta \\ \frac{\left(c-h_{T} E\right)|\cos \theta|+\sqrt{h_{T}^{2} E^{2}+h_{T}^{2}\left(2 c h_{T} E-c^{2}-E^{2}\right) \sin ^{2} \theta}}{\left(h_{T}^{2}-1\right) \sin ^{2} \theta+1} & \text { otherwise }\end{cases}
$$




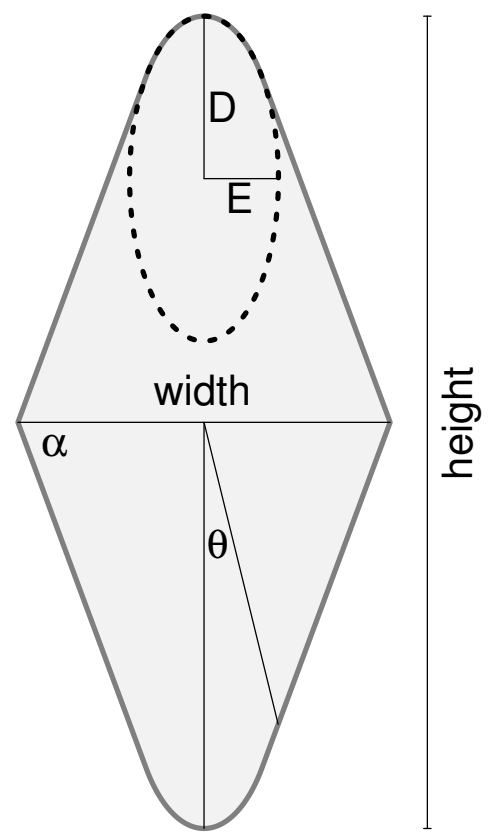

Figure 6.2: Schematic showing how the bicone geometry with a spheroidal tip is constructed. Here the parameters of the spheroid are chosen such that the first derivative of the geometry is continuous at the junction of the spheroid and the cone. The full shape is constructed by rotating this about the long axis. The angle $\theta$ is shown here, which is the angle from the $z$-axis. The angle $\alpha$ is the angle the sides of the shape make with the symmetry plane.

where

$$
\begin{aligned}
\beta & =-\tan ^{-1}\left(z / r_{p}\right)+\pi / 2 \\
z & =\frac{a+\left(c-h_{T} E\right) \frac{\tan \alpha}{h_{T}^{2}}}{\frac{\tan \alpha}{h_{T}^{2}}+\frac{1}{\tan \alpha}} \\
r_{p} & =a-\frac{z}{\tan \alpha} \\
h_{T} & =\frac{E^{2} \tan ^{2} \alpha-(a \tan \alpha-c)^{2}}{2 E(a \tan \alpha-c)} .
\end{aligned}
$$

Here $\beta$ is the value of $\theta$ at the point where the cone and the spheroid intersect, 
$\left(r_{p}, z\right)$ are the coordinates at that point, and $h_{T}$ is the ratio $D / E$, the aspect ratio of the spheroid at the tip. The semi-width and semi-height of the particle are $a$ and $c$ respectively. The free parameters in this model are $a, c, \alpha$ and $E$.

The dimensions of the bipyramids as measured by TEM were $45 \mathrm{~nm}$ along the long axis, and $20 \mathrm{~nm}$ along the short axis. In order to determine the parameters to be used to represent the spheroid, we modelled the optical response and modified the spheroid parameters to match the measured resonance in the extinction spectrum. This is discussed more in Section 6.1.3. An overlay of the final geometry to a TEM scan of some bipyramids is provided in Fig. 6.3.

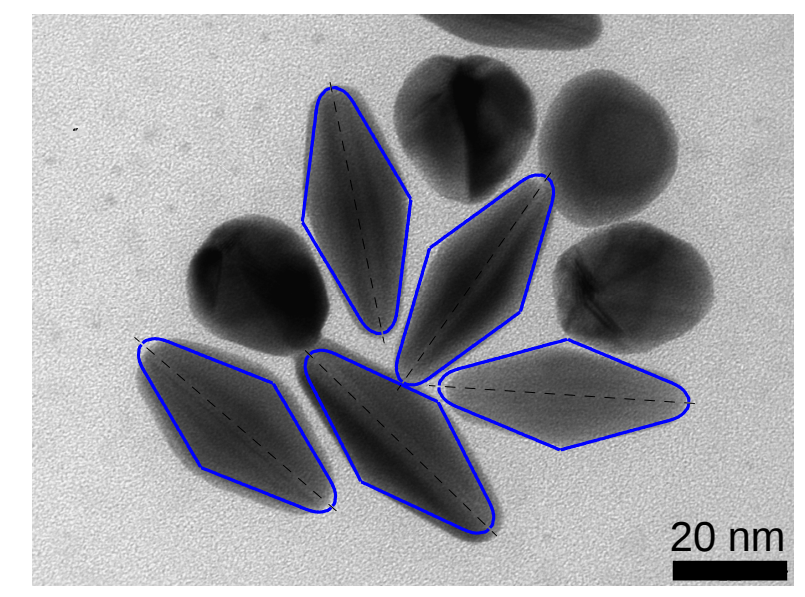

Figure 6.3: An overlay of the geometry we use onto a TEM image of the actual bipyramids used, showing that the model is a close approximation of the real shape. The technique used to synthesise the bipyramids also results in some spherical particles, whose resonance is far-removed from the bipyramid resonance, and hence the contributions of the two shapes may be resolved.

\subsubsection{Simulation Techniques}

For modelling the response of the bicones, we made use of COMSOL. Owing to the very subwavelength nature of the particles, we made use of the computationallyeasier electrostatics approximation, rather than solving the full-wave problem, after checking that results from the two methods were equivalent. In addition, as we are interested in the main dipolar resonance, along the long axis, we are able to 


\section{Application of Electromagnetic Calculations to Plasmonics}

use an axisymmetric simulation, which reduces the dimensionality of the problem. However, if we wanted to solve for incident polarisations different to the $z$-axis (along the long axis), then the axisymmetric simulation is not applicable, as the resulting field would then have a $\phi$-dependence. Similarly, for any full-wave simulations, the axisymmetric case is not applicable as the $k$-vector breaks the rotational symmetry.

The particles were considered to have the dielectric function of gold given in Ref. [83], and be embedded in water (with a refractive index of $n=1.33$ ). We neglected to model the CTAB layer, after simulations of its effect showed it to be small. Specifically, modelling a layer of refractive index $n=1.4$ tapering from $4 \mathrm{~nm}$ thick at the centre of the particle, to $0 \mathrm{~nm}$ thick at the tips, resulted in a resonance shift of only $4 \mathrm{~nm}$, and a reduction in the maximum calculated SERS intensity of $4 \%$.

Properties that are examined are the absorption cross section, and the maximum and average SERS enhancement factors. In the electrostatics approximation there is no scattering, so only the absorption cross section is relevant here. In addition, the quantity

$$
R=\frac{\mathrm{EF}_{\max }}{\langle\mathrm{EF}\rangle}
$$

is used to measure the localisation of the enhancement factor. This quantity is discussed in more detail in Section 6.3.

In order to calculate orientation-averaged enhancement factors, one approach is to model the resonance as a dipole, with the orientation-averaged values (of $F \approx|E|^{4}$ ) being one-fifth of the maximum values 84 . This method is valid only near the main (longitudinal) resonance, and not near to other resonances that the molecule has. The other approach is to carry out simulations with different incident directions, and average the results of all orientations. This precludes the 2D-axisymmetric simulation, as that requires the incident field is axisymmetric, which is only the case with the field along the $z$-axis. Thus, we resorted to 3D simulations, still using the electrostatics approximation. This allows the incident field to be rotated. The field on the surface as a function of angle is shown in Fig. 6.4, which compares the results from $F / 5$ and from averaging different incident directions. We can see that 


\subsection{Bipyramids and Bicones}

the $F / 5$ approximation is good for most angles, and specifically for the angles where the enhancement factor is high. The departure of the two curves from each other occurs in regions of low enhancement.

\subsubsection{Results}

The first step in modelling the bicones is to find the geometric parameters that best represent these particles, with the requirement that they also resemble visually the shapes from the TEM images. The total width and height of the bipyramids may be extracted directly from the images. The values that were found to match best are $E=2.27 \mathrm{~nm}$ and $D=4 \mathrm{~nm}$, with $\alpha=71^{\circ}$. These result in a resonance centred at a wavelength of $\lambda=658 \mathrm{~nm}$, to match experimental measurements.

Once the geometric parameters have been found, then the enhancement factors may be examined. In most cases we would simply look at the simplest approximation of the SERS EF, $|\vec{E}|^{4} /\left|\vec{E}_{0}\right|^{4}$, but here we are comparing the results to some experimental data, specifically data with a known Raman shift. The laser wavelength used was $633 \mathrm{~nm}$, and the Raman mode examined had a Raman shift of $1620 \mathrm{~cm}^{-1}$, which for that incident laser corresponds to a wavelength of $705 \mathrm{~nm}$. In this case, the SERS EF is better represented as

$$
F=\frac{\left|\vec{E}_{633}\right|^{2}\left|\vec{E}_{705}\right|^{2}}{\left|E_{0}\right|^{4}}
$$

which requires simulations at two different wavelengths 1 Here $\vec{E}_{633}$ is the local electric field for excitation at $\lambda=633 \mathrm{~nm}$, and similarly for $\vec{E}_{705}$. By reciprocity, the excitation and emission processes share the same enhancement factor. A plot of the enhancement factor in the region around the bicone is shown in Fig. 6.5. The maximum enhancement factor from these simulations is $F=1.29 \times 10^{7}$, and the average is $\langle F\rangle=2.41 \times 10^{5}$.

The ratio $R$ as calculated from these simulations is $R_{\text {simulation }}=53.5$. This is in

\footnotetext{
${ }^{1}$ In the electrostatics approximation, the only difference between these simulations is the dielectric function used for the gold.
} 


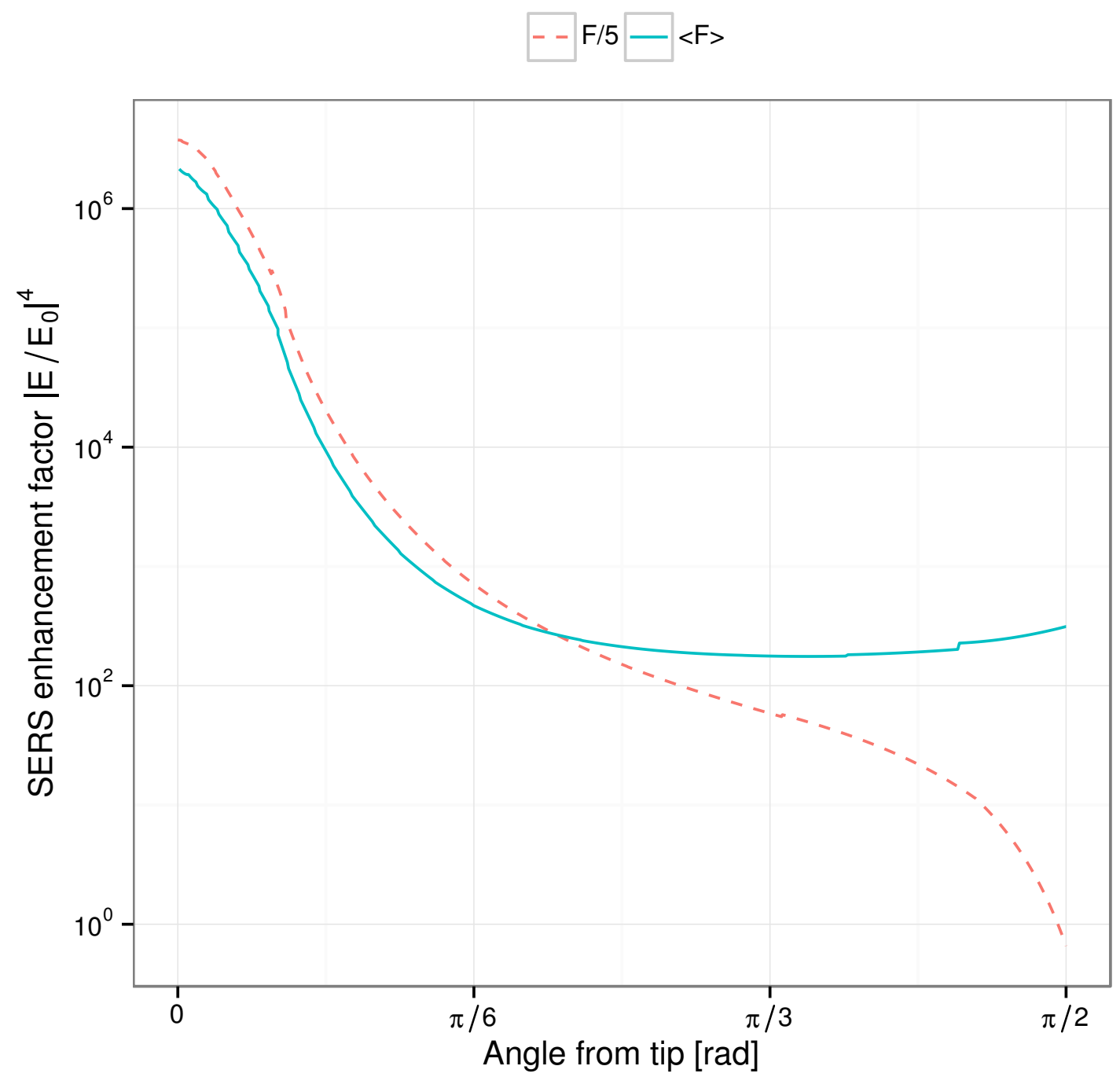

Figure 6.4: The angular behaviour of the orientation-averaged SERS enhancement factor on a bicone. Here the dashed line is from the $F / 5$ approximation, where $F$ is the EF from excitation with $\vec{E}$ along the $z$-axis, while the solid line is for averaging over 20 different incident directions, evenlyspaced in $\cos \theta$. The two lines separate at large angles as the resonance along the minor axis is excited only for the orientation-averaged result, while the $F / 5$ approach does not excite that resonance at all. 


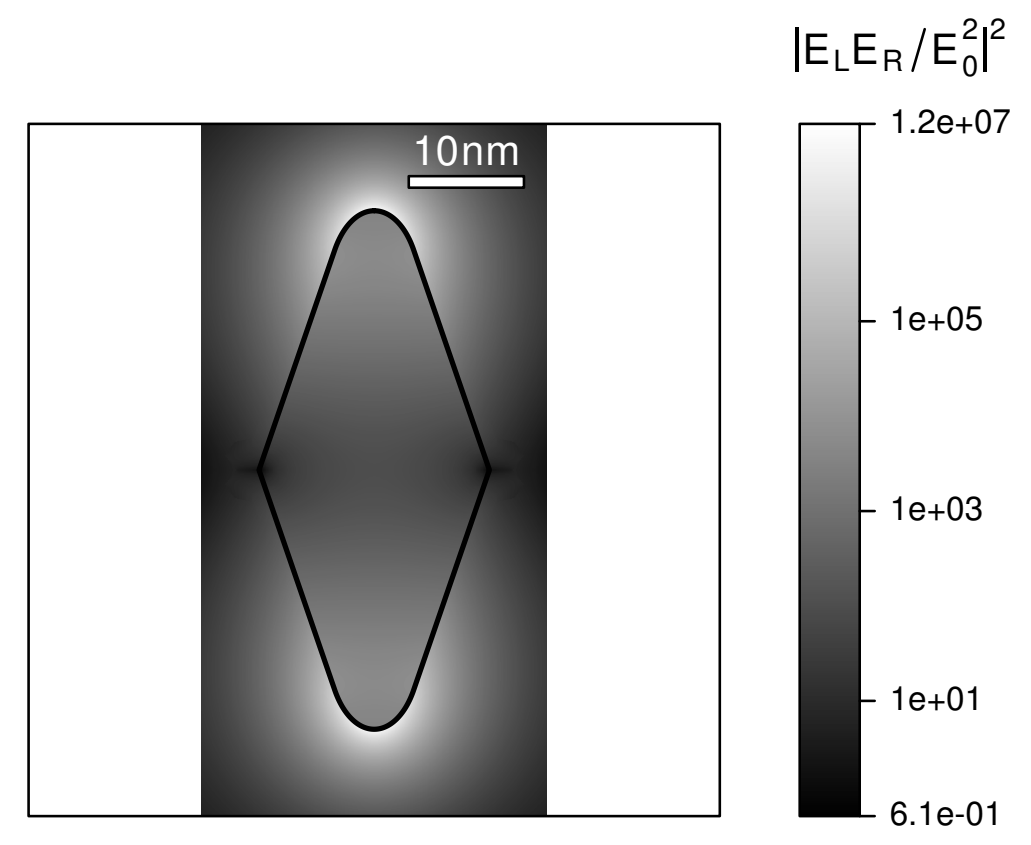

Figure 6.5: The SERS enhancement factor $F$ around the bipyramid, as calculated by COMSOL. This is calculated as $F=\left|\vec{E}_{L}\right|^{2}\left|\vec{E}_{R}\right|^{2} /\left|\vec{E}_{0}\right|^{4}$, for a laser wavelength of $633 \mathrm{~nm}$, and a Raman shift of $1620 \mathrm{~cm}^{-1}$, corresponding to a wavelength of $705 \mathrm{~nm}$. 


\section{Application of Electromagnetic Calculations to Plasmonics}

contrast to the experimental estimate of $R_{\text {experiment }}=7.7$, from SERS measurements. For a discussion of experimental determination of the +relevant enhancement factors, see Ref. [85]. The +difference in the values of $R$ is attributed to the CTAB layer, which prevents the probe molecules adsorbing over most of the particle. If we instead define $R$ such that the average EF is averaged only over spheroidal tip region, then we get a value of $R_{\text {tip only }}=4.2$. That this is slightly lower than the experimental value might suggest that the particles are able to adsorb over a larger region than just the tip. A value of $R=1$ would indicate that all molecules experience exactly the same enhancement factor, such as if they could only adsorb directly at the hot-spot.

\subsection{Chains of Spheres}

This section concerns simulations done in support of the work reported in Ref. [5]. This paper concerns surface-enhanced fluorescence measurements of dye-labelled DNA on a substrate of aggregated silver colloids. As it was noted that the extinction spectrum peak red-shifted as the nanoparticles aggregated before reaching a steady position, we attempted to replicate this with simulation results. For this, COMSOL was used, and full-wave simulations were run. This requires a 3D simulation.

For simplicity, instead of aggregation of clusters of spheres, we modelled the lengthening of chains of spheres, from two spheres to 8 spheres. The spheres have a radius of $17 \mathrm{~nm}$, and the gap between neighbouring spheres is $1.5 \mathrm{~nm}$. The dielectric function of silver used is that from Ref. [10], and the particles are embedded in water, modelled as having a refractive index of $n=1.33$.

In order to eliminate the effects of reflections of the scattered wave off the edges of the simulation volume, a perfectly matched layer (PML) is used. This layer is designed so that the scattered field that is incident on this layer is adsorbed with no reflections. This is so that the finite size (and hence edges) of the simulation volume have no effect on the simulation results.

In order to test the accuracy of the simulations, the results of light scattering by a dimer were compared to results obtained from generalised Mie theory. A comparison 
of the absorption and scattering efficiencies, as well as the value of $|E|^{2}$ at the centre of the gap, from the two methods is shown in Fig. 6.6. By comparing these, we may be confident that the results from the COMSOL simulations are as accurate as those from Mie theory.

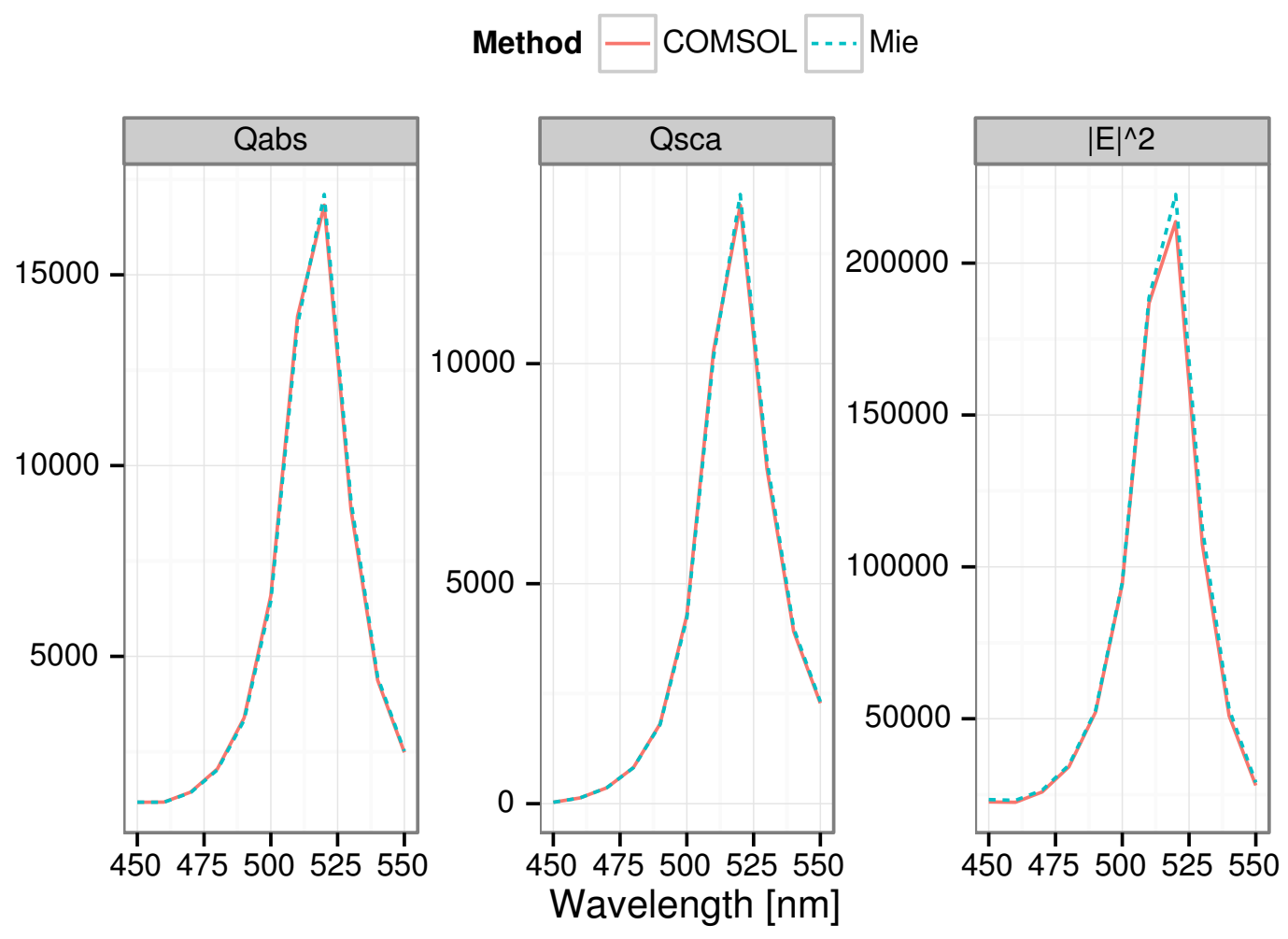

Figure 6.6: A comparison of the absorption, scattering and local field of a dimer of silver spheres, radius $17 \mathrm{~nm}$, with a $1.5 \mathrm{~nm}$ gap, as calculated by generalised Mie theory and a full-wave simulation in COMSOL with a PML. This case, of a silver dimer at resonance, with a small inter-particle gap, is one of the more difficult simulations, as silver has low absorption here, and so the resonance is sharp.

These results, where fully numerical results from COMSOL show good agreement with analytical methods, even for challenging cases of sharp resonances, demonstrate that COMSOL is able to solve full-wave problems even in challenging cases, and in situations where the numerical methods run into numerical problems, such as long 


\section{Application of Electromagnetic Calculations to Plasmonics}

chains of spheres. Also, as COMSOL does not take the symmetry into account, it is not constrained to solving problems that have this symmetry. However, the price to pay for this generality is the speed of the calculations. For the dimers in Fig. 6.6, on a quad-core Intel Xeon W3520 $2.67 \mathrm{GHz}$ desktop with $18 \mathrm{~GB}$ of RAM, COMSOL $4.3 \mathrm{~b}$ took 41 minutes to solve the problem (for all wavelengths), with 1507354 degrees of freedom. Calculating the scattered and absorbed power took additional time. In contrast, MATLAB R2012a on the same computer takes 15 seconds using generalised Mie theory codes.

The calculated extinction spectra for a variety of chains of spheres of different lengths is provided in Fig. 6.7. These silver spheres embedded in +water $(n=1.33)$ each have a radius of $17 \mathrm{~nm}$, and the +inter-sphere gap is $1.5 \mathrm{~nm}$. Of interest here is that as the number of spheres increases, the position of the main dipolar resonance appears to be approaching some limit, after which the addition of more spheres to the chain will not shift the resonance. This qualitatively supports the experiments observations in [5].

\subsection{EF Localisation}

In several fields, there is much interest in obtaining large electromagnetic enhancement factors (EFs). One such example is in SERS, where the enhancement in the Raman signal goes roughly as $\left|E / E_{0}\right|^{4}$ [32]. Other uses include in nonlinear processes (such as second-harmonic generation) [86]. As a result, quite some effort is put into developing systems which generate large electric fields (or large field enhancements, in the case of SERS). Focusing on the case of SERS, there is a wealth of literature of reports of SERS substrates with higher and higher EFs[87 91]. These include attempts to obtain both large maximum EFs as well as large average EFs. However, in this chapter, we focus not on attaining the largest possible enhancements, but rather on examining the manner in which the enhancement is distributed[4]. Typically in these situations, there are regions with much higher enhancement that other regions. These are referred to as "hot-spots". Continuing the theme of this thesis looking at light scattering by particles, the SERS systems considered here are single or multi- 


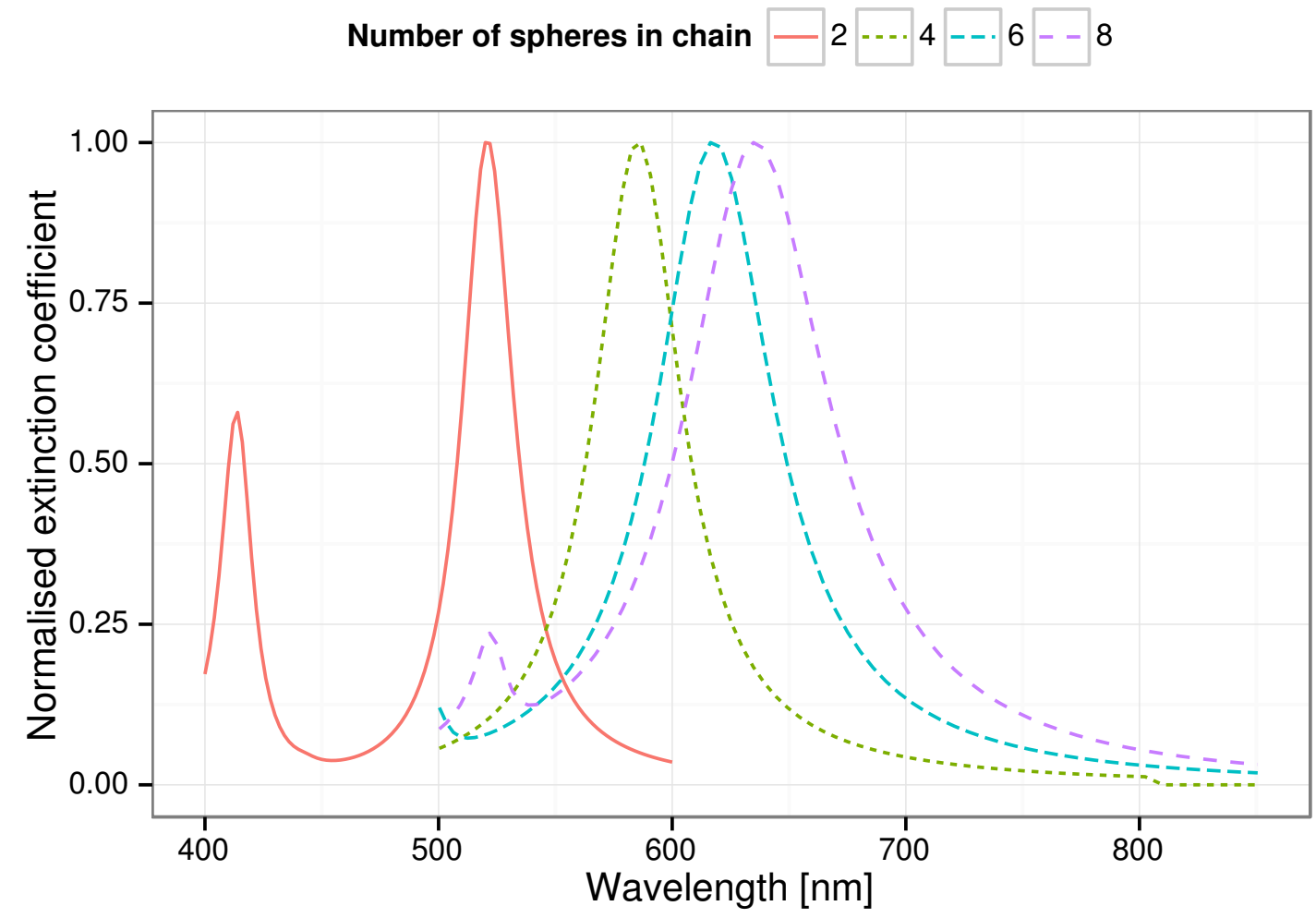

Figure 6.7: The extinction spectra of chains of spheres of varying lengths, normalised to the maximum value for each chain. These are silver spheres in water $(n=1.33)$, with a gap between spheres of $1.5 \mathrm{~nm}$, and a radius of $17 \mathrm{~nm}$. These are from full-wave electromagnetic simulations carried out in COMSOL. 


\section{Application of Electromagnetic Calculations to Plasmonics}

ple particles, and not an extended substrate. This represents a typical experiment carried out in a solution of colloids, and while some of the results may carry over to other systems (such as roughened silver surfaces, or lithographically-produced arrays of particles on a support), some care should be taken.

There are several obvious ways to change the EFs in the vicinity of a particle. These include changing the wavelength, incidence direction or polarisation of the incident light, as well as the size, shape or material of the particles. Typically making particles 'sharper' will lead to larger EFs, as will using wavelengths closer to electromagnetic resonances.

\subsubsection{Different Metrics}

There are a variety of different metrics that we may consider when attempting to evaluate the hot-spot, which provide a variety of different information. In this section are provided a brief description of some of these metrics, including how they are calculated, and what they mean physically. Looking at the field distribution by considering the field at every point leads to a practically-challenging analysis problem. Hence, it is preferred to have some single-valued metric, which simplifies analysis.

\section{Common Metrics}

These metrics are the simplest, in that they are simple to calculate, and have an easily-accessible physical meaning. They also measure the strength of the response, and do not make a statement about the localisation of the hot-spot.

The first is simply the maximum enhancement factor, $\mathrm{EF}_{\max }$. This is the maximum of the enhancement factor on the surface of the particle, the location of which depends on the details of the shape and the excitation.

The second is the average value of the enhancement factor on the surface, $\langle\mathrm{EF}\rangle$. This represents the average enhancement factor experienced by an average molecule on the surface (in the case of SERS), assuming random surface adsorption. This is 


\subsection{EF Localisation}

computed as

$$
\langle M\rangle=\int_{S} \frac{M(\vec{r}) \mathrm{d} S}{S}
$$

where $S$ is the surface area of the particle, and $M$ is the enhancement factor of interest (such as $|E|,|E|^{2}$, etc.).

In SERS experiments, it is the average enhancement factor $\langle\mathrm{EF}\rangle$ that is typically measured. In the case of single-molecule SERS, information is instead provided about the maximum enhancement factor, $\mathrm{EF}_{\max }$. However, neither of these metrics provide information about the distribution of enhancement factors..

\section{Localisation Metrics}

The previous section looks at metrics that do not consider the localisation of the hot-spot. In this section, we examine several metrics that do not respond to the size of the enhancement, but more how it is distributed. These metrics are focused on understanding SERS enhancement factors (so look at $\left|E / E_{0}\right|^{4}$ ), but are applicable more generally also. All of this study considers the metrics at the main dipolar resonance of the particle being considered.

The first metric here is the ratio of maximum to average enhancement factor,

$$
R=\mathrm{EF}_{\max } /\langle\mathrm{EF}\rangle
$$

Clearly, if we are able to change the enhancement factors such that all the values, say, double (for example by changing the material), then this metric remains unchanged. In terms of SERS scatterers, this represents the number of average $(\sim$ randomly positioned) molecules that produce the same signal as one molecule that experiences the maximum enhancement. This metric is the one shown in Ref. [92], where it is shown that, in the case of spheroids at their dipolar resonance, this ratio $R$ is independent of material or size, but depends only on the aspect ratio of the particles.

The next metric looks at what fraction of the area of the particle is responsible for some fraction of the signal. This metric, $a_{x}$, where $x$ is some percentage, is 
the fraction of the area responsible for $x \%$ of the signal. Typically we look at $a_{80}$, the fraction of the area from which $80 \%$ of the signal from randomly-positioned molecules arises. This is calculated from

$$
a_{80}=\int_{\Omega} \frac{\mathrm{d} S}{S}
$$

where we choose $\Omega$ such that

$$
\int_{\Omega} \frac{M(\vec{r}) \mathrm{d} S}{S}=0.8 \int_{S} \frac{M(\vec{r}) \mathrm{d} S}{S} .
$$

Here the choice of $\Omega$ should be the smallest possible area that fulfils these conditions (in order to minimise the value of $a_{80}$ ), and $\Omega$ need not be a connected region.

\subsubsection{Shape Effect}

Following on from [92], it seems worthwhile to investigate the effect of shape on the enhancement factor distribution. The main aspects of the shape that can be categorised easily are the global aspect ratio (ratio of maximum to minimum distance from the centre) and the curvature of the surface at the hot-spot. There is some intuitive expectation that the local curvature (at the hot-spot) is responsible for increasing the localisation. In general, pointier shapes lead to higher degrees of localisation, and also a higher maximum enhancement. In order to investigate this behaviour, shapes which allow us to examine the different contributions are needed. The limitations of spheroids here is that the global aspect ratio determines the curvature: the two are not independent. The following sections contain descriptions of the different classes of shapes that are used to investigate the effects of curvature. For this study, all of the particles used were prolate (higher than they are wide).

\section{Spheroids}

The first shape considered was the spheroid, as used in [92]. Some examples of spheroids are depicted in Fig. 6.8. However, as mentioned before, for spheroids it 


\subsection{EF Localisation}

is not possible to independently change the aspect ratio and curvature, so we are limited in our ability to decouple the two. The equation for the geometry of a spheroid is (in spherical coordinates)

$$
r(\theta)=\frac{a c}{\sqrt{a^{2} \cos ^{2} \theta+c^{2} \cos ^{2} \theta}} .
$$

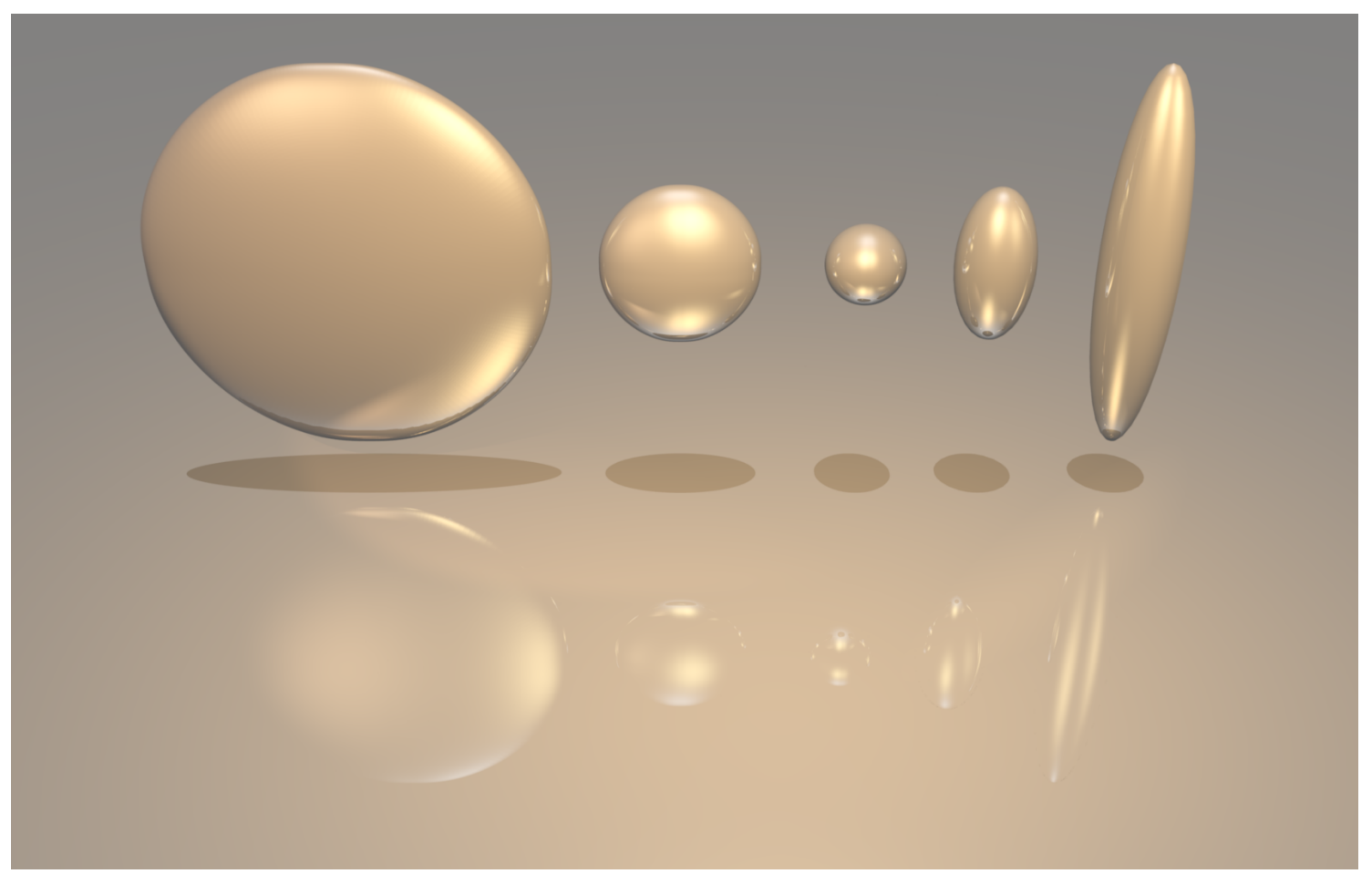

Figure 6.8: A depiction of a collection of spheroids. The two left-most are oblate spheroids, the centre object is a sphere, and the two on the right are prolate spheroids. The aspect ratios are (from the left) $5,2,1,2,5$.

\section{Bicones}

The next class of shapes are those that are the focus of Section 6.1, and are inspired by the colloids used in [2], bipyramids. An electron micrograph showing an example of these particles is given in Fig. 6.1. In this study, however, we look at bicones, which differ from the bipyramids in that they are not faceted, they have smooth sides. This is for ease of simulations, as the bicones have axial symmetry. 


\section{Application of Electromagnetic Calculations to Plasmonics}

The model of the bicone geometry is given in Fig. 6.2. Here, the bicone is made of two cones arranged base-to-base, where the tip is replaced by a truncated spheroid in such a way that the first derivative of the surface is continuous. The equation for this geometry is provided in Section 6.1.1. This geometry allows two additional degrees of freedom over a simple spheroid. There are 4 parameters, the width and height of each of the overall shape, and the spheroid at the tip. A collection of bicones with different parameters is shown in Fig. 6.9.

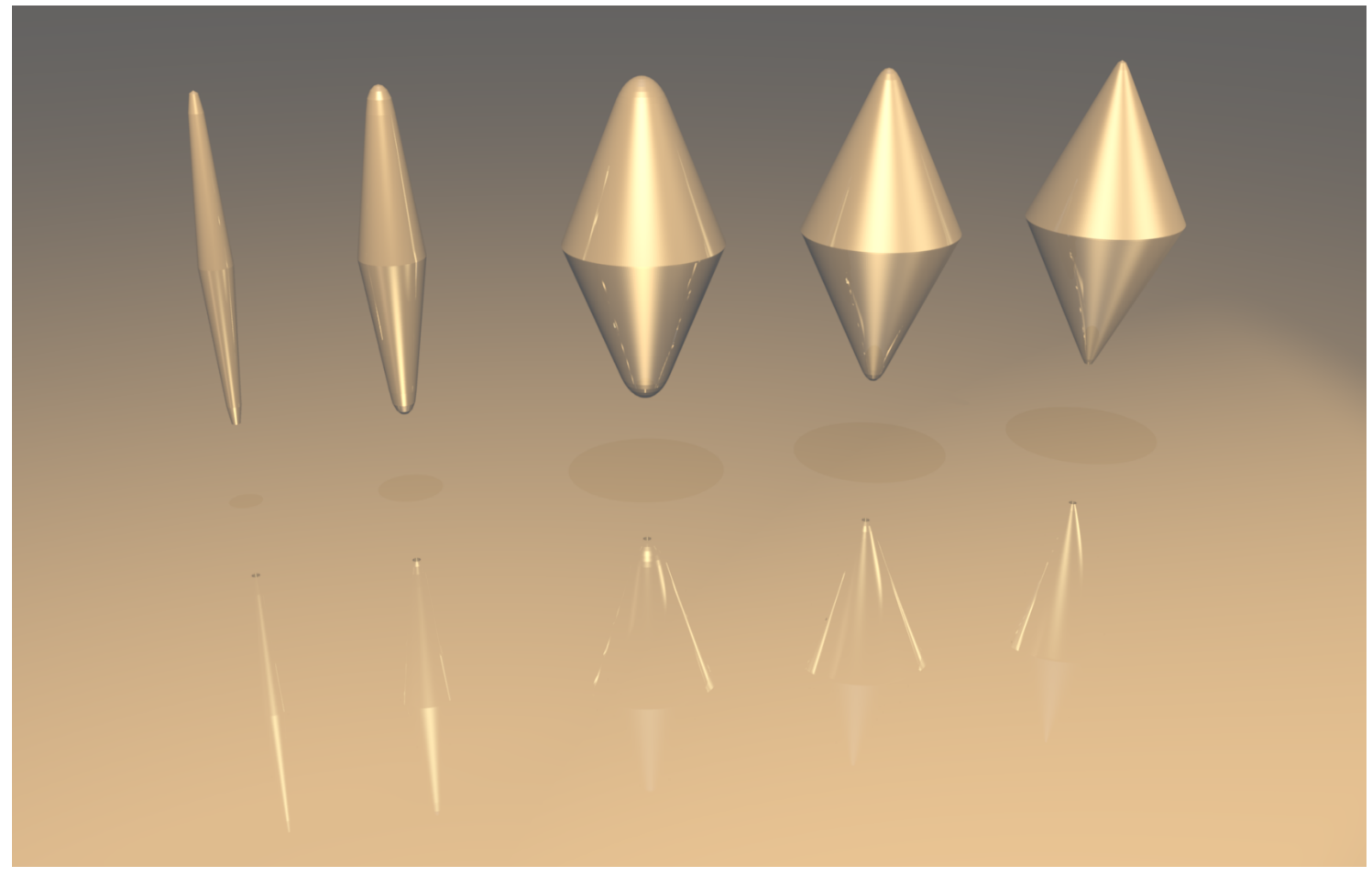

Figure 6.9: A selection of bicones with different parameters. The centre bicone has a global aspect ratio of two, and an aspect ratio of the spheroid that makes up the tip of two. To the right, the aspect ratio of the spheroid at the tip increases to five and then 10, while the global aspect ratio remains constant. To the left, the tip aspect ratio remains at two, while the global aspect ratio increases to five and then 10 . 


\subsection{EF Localisation}

\section{Modified Spheroids}

Here we look at a class of shapes that are a modification of the spheroid shape designed to add another parameter. The form of this is given by

$$
\begin{aligned}
& f(\theta)=\left(\cos ^{2} \theta\right)^{n} \\
& r(\theta)=\frac{1}{\left[c^{-1 / p} f(\theta)+a^{-1 / p}(1-f(\theta))\right]^{p} .}
\end{aligned}
$$

Here $n$ is an integer (restricted here to 1 or 2 ), and $p$ is between 0 and 1 . As we can see in Fig. 6.10, these shapes can have a variety of tip curvatures, while maintaining the same overall aspect ratio.

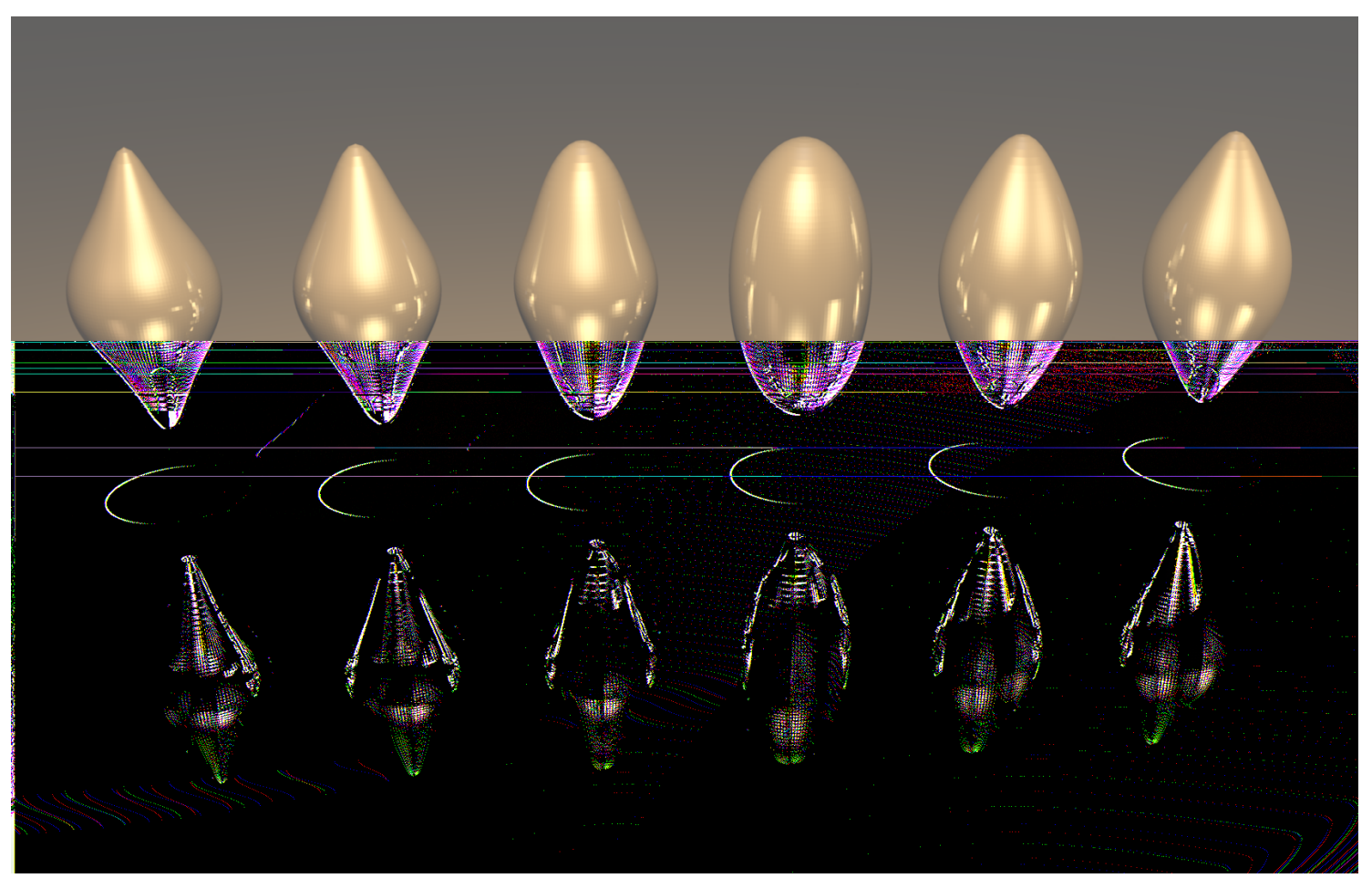

Figure 6.10: A selection of some modified spheroids, as described by Eq. 6.14). The three leftmost shapes have $n=2$, while the three on the right have $n=1$. The values of $p$ are, from the left, $0.2,0.25,0.5,0.5,0.25,0.2$. All of these shapes have an aspect ratio of two. The $n=1, p=0.5$ (fourth from the left) shape is identical to a normal spheroid. 


\subsubsection{Effect of Different Parameters}

We wish to understand the effects of different parameters on the different metrics we have about the enhancement. We start by looking at the different metrics in the case of spheroids. In this case, we are unable to independently alter the curvature of the tips and the aspect ratio. However, it is interesting to have these results to compare to the other shapes.

Fig. 6.11 shows how the metrics $R, F_{\max }=\left|E_{\max } / E_{0}\right|^{4}$, and $a_{80}$ vary as the aspect ratio is varied. Here, there are two different sizes for spheroids, and also both gold and silver spheroids. The simulations for each case were carried out at the dipolar plasmon resonance, to match possible experimental conditions. As can be seen in the figure, the value of $F_{\max }$ varies for the different sizes and materials, but the metrics which are a measure of localisation, $R$ and $a_{80}$, show essentially no variation with these parameters. They are dictated solely by the aspect ratio of the particles.

The next shape to be considered is the bicone, where it is possible to independently change the curvature at the tips and the overall aspect ratio of the particle. Fig. 6.12 shows how the metrics change as the particle is altered, either by changing the curvature of the tip, or by changing the aspect ratio of the particle as a whole. For this shape, we can see that changing the curvature at the tips (which are the locations of the hot-spots) leads to large changes in $F_{\max }$, as well as the localisation metrics $R$ and $a_{80}$. In contrast, changing the overall aspect ratio, but leaving the tips unchanged, leads to much smaller changes in the properties. Figure 6.13 shows the enhancement of $|E|^{4}$ in the vicinity of the bicone, for tip aspect ratios of two and 10. This shows that the hot-spot is a lot more localised for the case where the tip is sharper, even though the overall aspect ratio is unchanged.

This confirms what is more-or-less expected, that changes in the particle in the vicinity of the hot-spot lead to changes in the behaviour of that hot-spot (including localisation), while changes in the overall shape of the particle influence more the location of the resonance. This is the case here, where the resonance position varies substantially when altering the overall shape, but by less than $40 \mathrm{~nm}$ covering the entire range of modifying the tip curvature. 


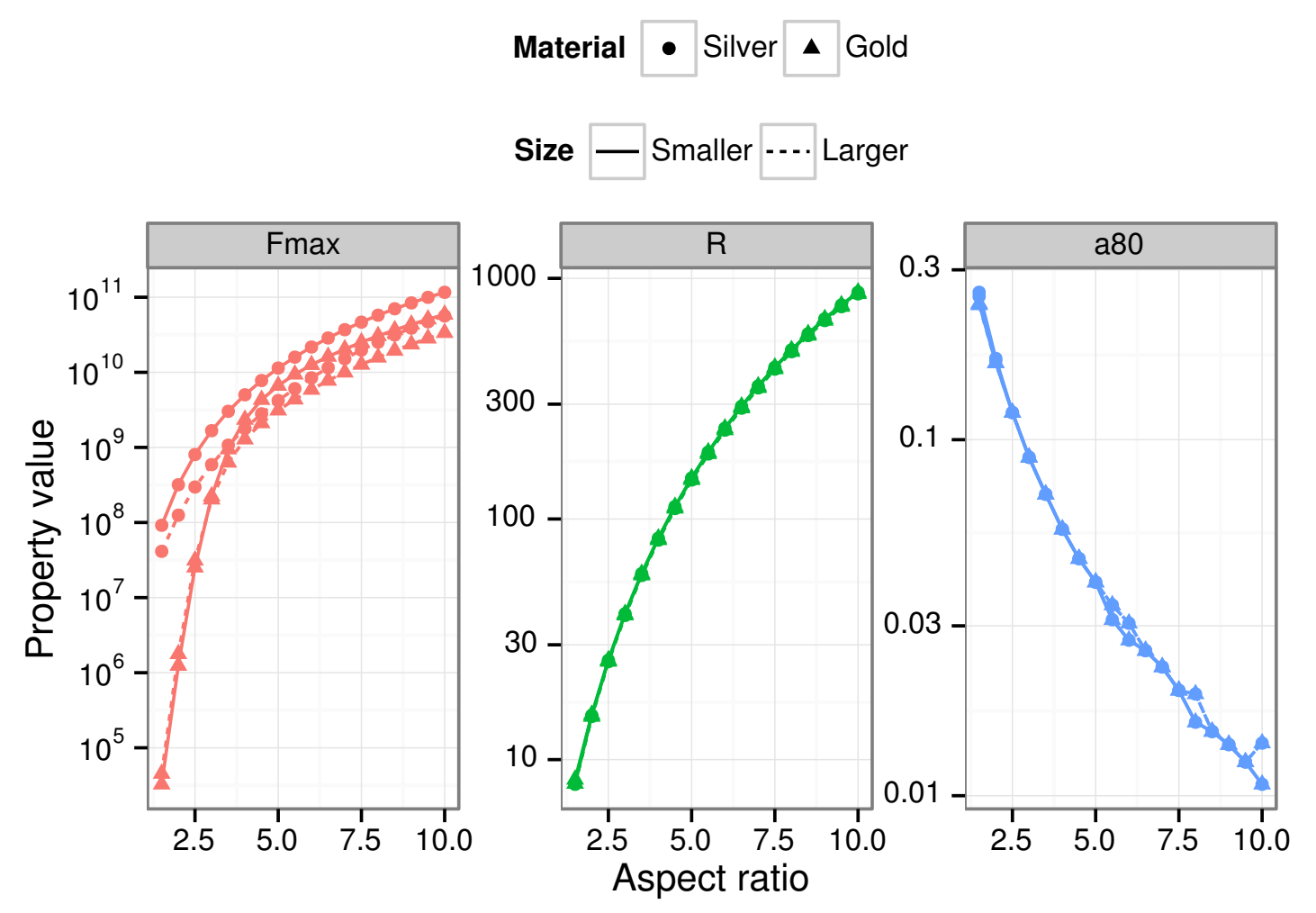

Figure 6.11: The variation of properties $F_{\max }, R$ and $a_{80}$ for spheroids of different size (minor semiaxis lengths of 1 (solid) and $10 \mathrm{~nm}$ (dashed)), made of gold (triangles) and silver (circles). Each data point is for a simulation carried out at the dipolar resonance for that particle. Note that the panels for $R$ and $a_{80}$ do contain four lines, that happen to be very close to each other. The behaviour of $R$ here replicates the results of Ref. [92]. 


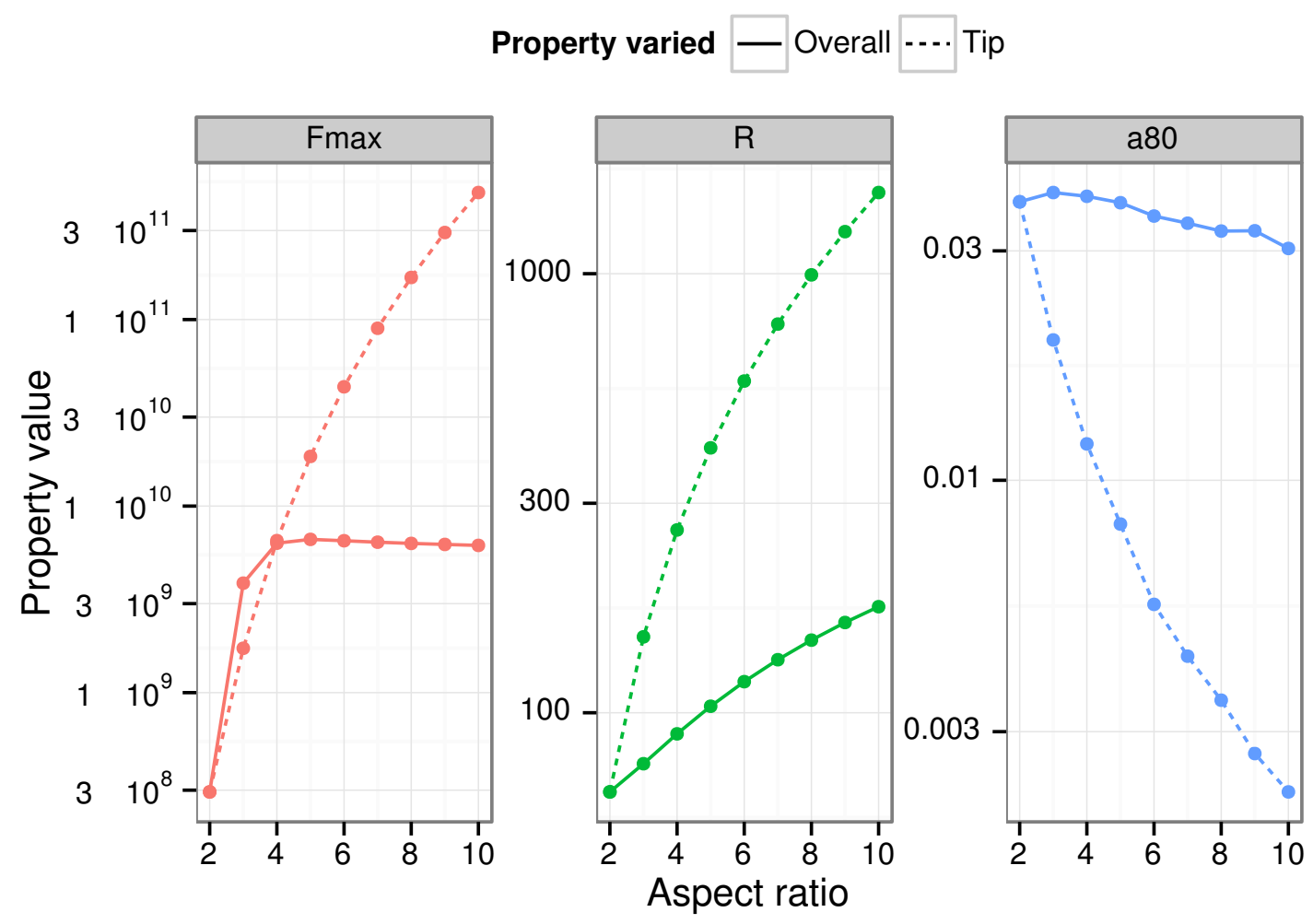

Figure 6.12: This shows how the different properties $F_{\max }, R$ and $a_{80}$ for a gold bicone vary as different geometric parameters are changed. Here dashed lines represent changing the aspect ratio of the spheroid at the tip of the bicone, leaving the overall aspect ratio at two. Solid lines signify leaving the spheroid tip aspect ratio at two, and altering the overall aspect ratio of the particles. These simulations were carried out in COMSOL, using the electrostatics approximation. Each simulation was carried out at the resonant frequency, which varied from $636-672 \mathrm{~nm}$ for changing the tip properties, and from $636-1541 \mathrm{~nm}$ for changing the overall aspect ratio. 


\subsection{EF Localisation}

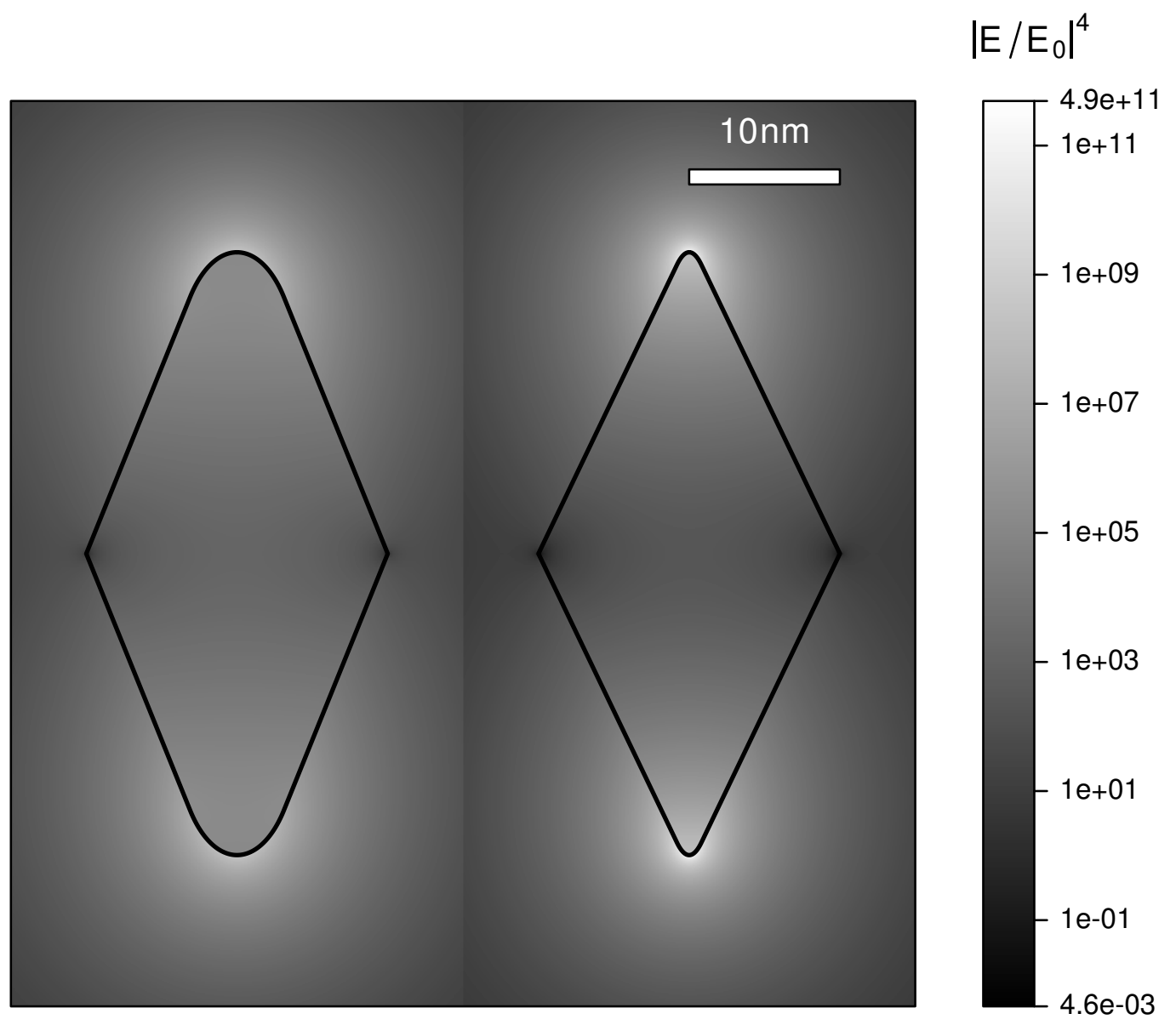

Figure 6.13: The enhancement of $\left|E / E_{0}\right|^{4}$ in the vicinity of two of the bicones in Fig. 6.12, those with a overall aspect ratio of two, and aspect ratios of the tip spheroids of two (left) and 10 (right). This is carried out at the resonance position for each of the shapes, $636 \mathrm{~nm}$ for the left one, and $672 \mathrm{~nm}$ for the right one. 


\section{Application of Electromagnetic Calculations to Plasmonics}

We are also able to look at the modified spheroids, where it is possible to modify the curvature of the tips while leaving the overall aspect ratio the same. For this, we examined a selection of different particles, and looked at the extinction efficiency (a typical far-field measurement), as well as the behaviour of $F_{\max }$ around the hotspot at resonance. These simulations were carried out in COMSOL, using a fullwave electromagnetic simulation. Initially, we begin with the collection of shapes presented in Fig. 6.14(a), These all have the same overall aspect ratio $h=2$, but the curvature at the tips is different. The far-field extinction efficiency is given in Fig. 6.14(b), and the positions of the resonances are all slightly different. All of the shapes also have different near-field behaviour, shown in Fig. 6.14(c), examining $F$ as a function of the angle from the $z$-axis.

In contrast are the shapes in Fig. 6.15(a). Superficially, these shapes look the same as their counterparts from Fig. 6.14(a), However, they have one key difference. The aspect ratio of these shapes was varied slightly, between $h=2$ and $h=1.83$, such that the resonance location for all of the shapes is the same. This is shown in Fig. 6.15(b), where the normalised extinction efficiency of all of the different shapes is indistinguishable. There is a difference in the unnormalised value, but experimentally, the absolute value of this is not easily obtainable. Hence, these are shapes that, from this resonance, are not distinguishable from extinction measurements. The shapes here are also similar, such that attempting to distinguish them on an electron micrograph would be difficult. Hence, the two far-field methods readily available to characterise these particles would not be a reliable means of distinguishing them. However, if we examine their near-field behaviour in Fig. 6.15(c), their distribution on the surface differs.

This highlights a key point to be wary of when attempting to model experimental systems, especially when it is the near-field that is of interest. Simply ensuring that the far-field behaviour, be it spectral or morphological, matches the experimental system, might well be insufficient to guarantee the near-field behaviour will match. Sadly, there is no obvious way around this, without resorting to near-field probes to test the near-field experimentally, which would greatly complicate studies. 


\subsection{EF Localisation}
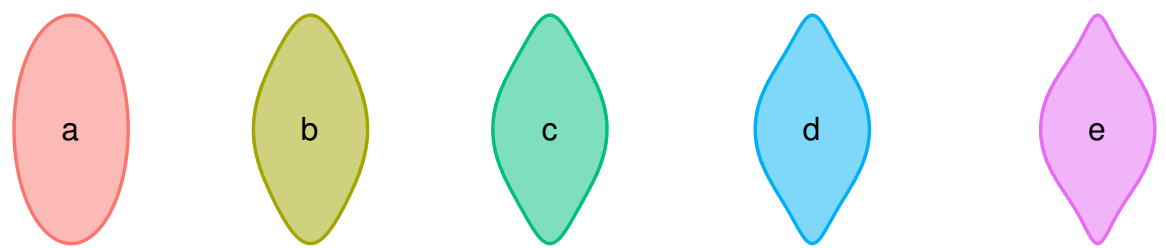

(a) The modified spheroids used here, all have the same aspect ratio $h=2$.

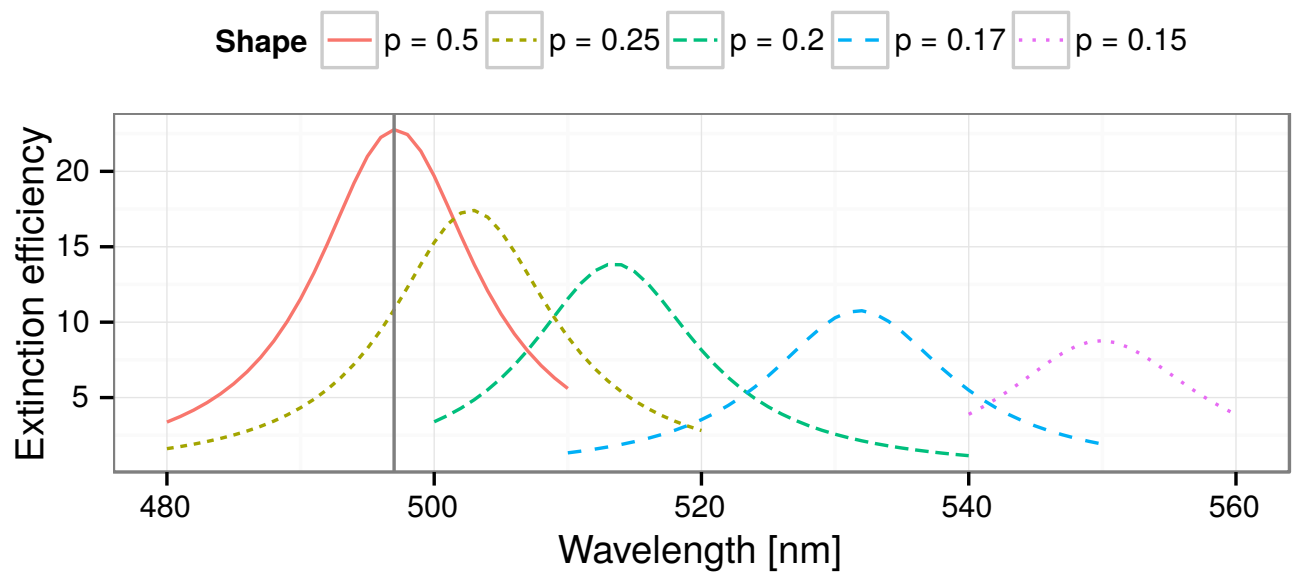

(b) The extinction efficiency for each of the shapes, showing a different resonance position for each shape. The vertical bar shows the position of the electrostatics spheroid resonance.

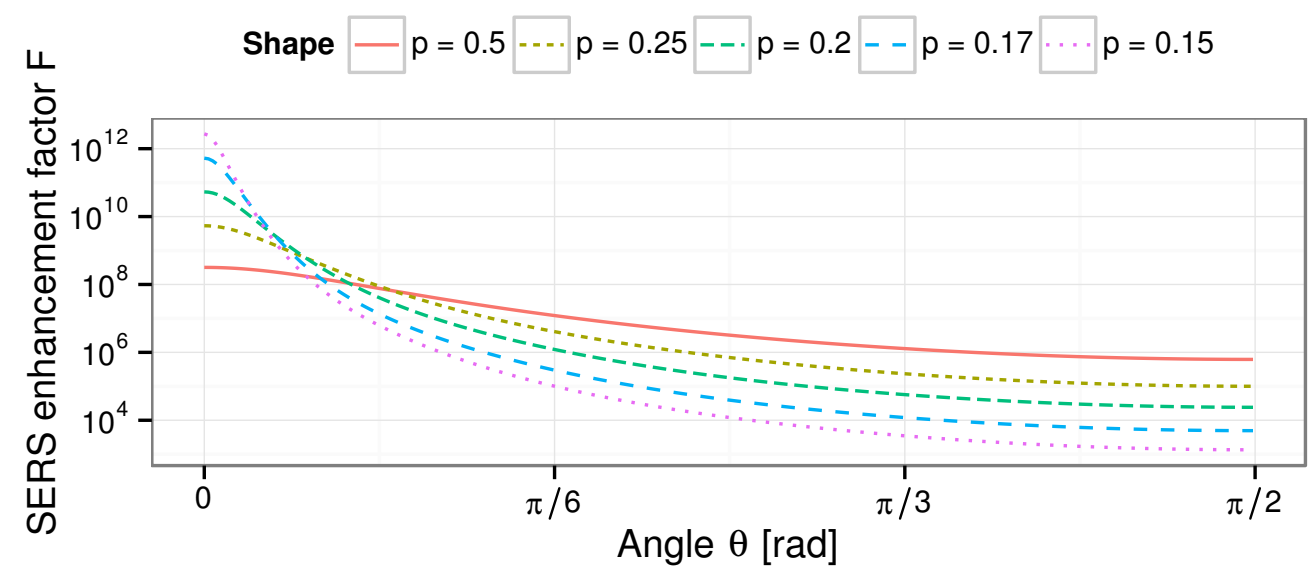

(c) The SERS enhancement factor on the surface of the particle for each of these shapes at their resonance.

Figure 6.14: Various properties of different modified spheroids. Shown are (a) outlines of the shapes, (b) extinction efficiency spectra for each shape and (c) the SERS EF near the hot-spot for each of the shapes at their resonance. 

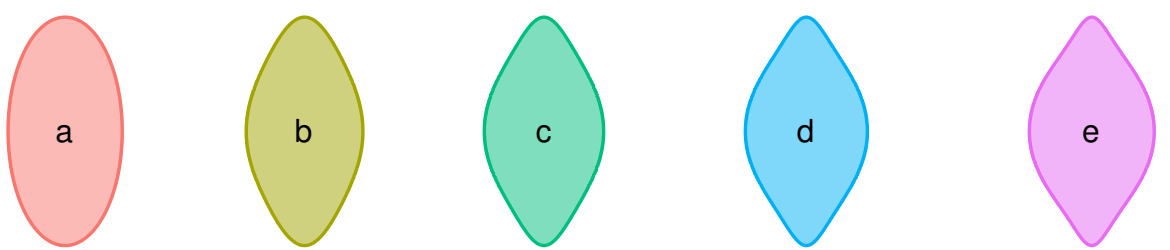

(a) The modified spheroids used here, all with slightly different aspect ratios.

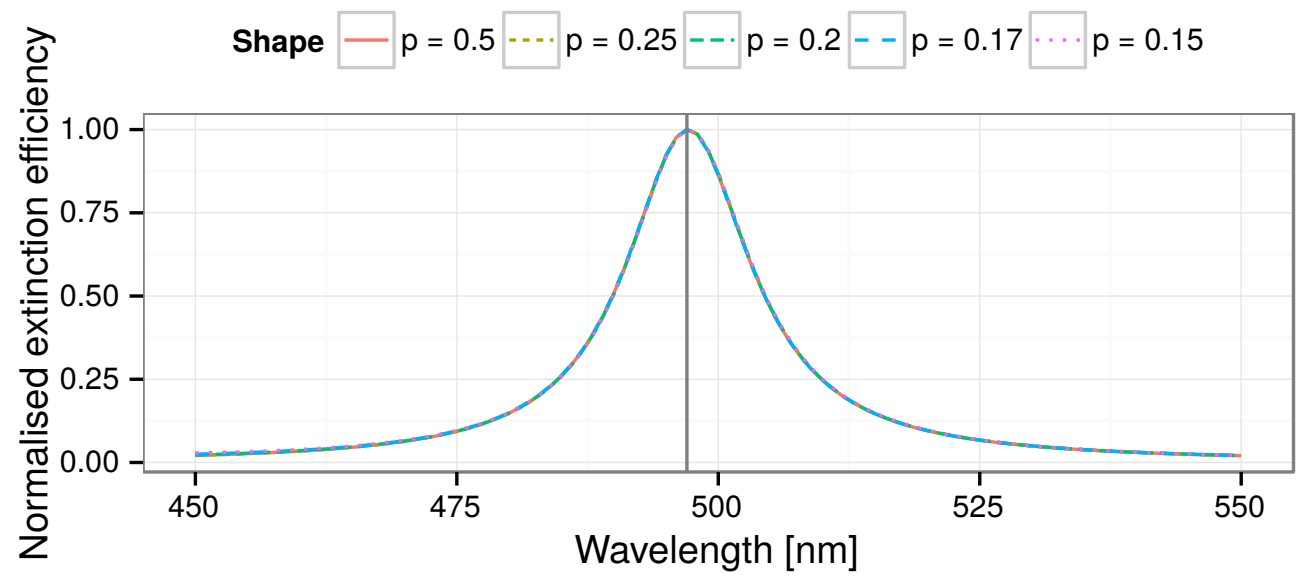

(b) The normalised extinction efficiency for each of the shapes, showing the same resonance position for each shape. There are five lines overlaid here. The vertical bar shows the position of the electrostatics spheroid resonance.

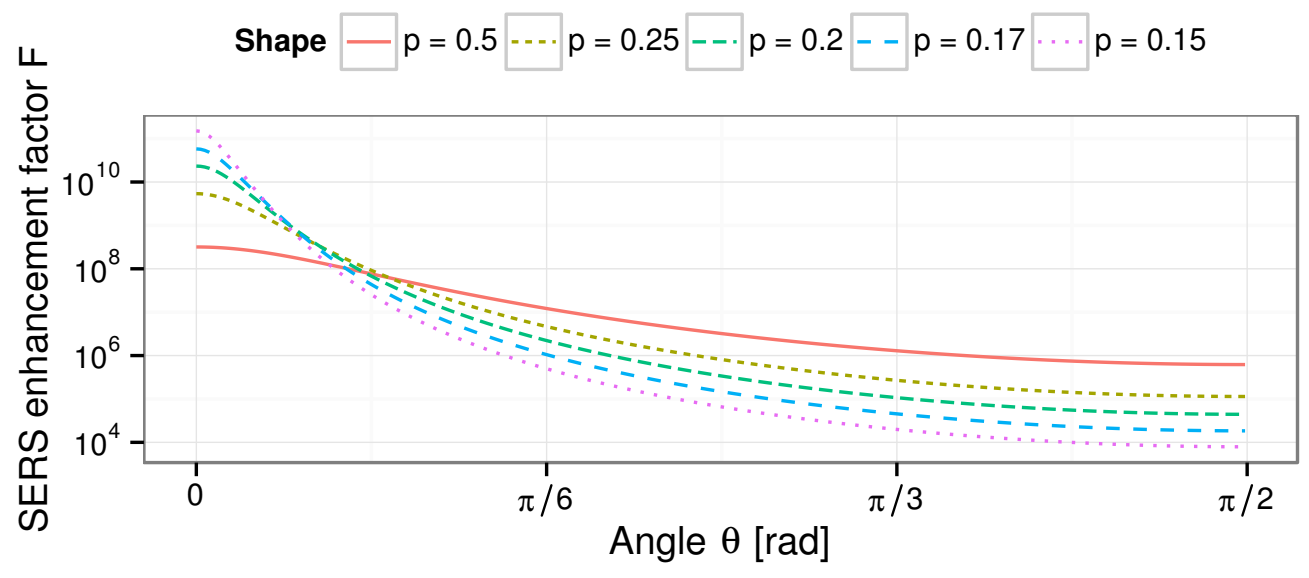

(c) The SERS enhancement factor on the surface of the particle for each of these shapes at their (joint) resonance.

Figure 6.15: Various properties of different modified spheroids having the same resonance. Shown are (a) outlines of the shapes, (b) normalised extinction efficiency spectra for each shape and (c) the SERS EF near the hot-spot for each of the shapes at their resonance. 


\subsection{EF Localisation}

\subsubsection{Implications of Different Metrics}

It is worth considering what metrics we might want to optimise, when trying to select (or design) a substrate for a SERS experiment (for example). To answer this, we must consider what we are trying to gain from using SERS, as opposed to 'normal' Raman spectroscopy. While it is true that SERS may be used to probe the interactions of the target molecule with the surface [28, 93], it is often used as a means of increasing the signal. To this end, there are two main approaches that are used. One is to maximise the signal collected, to detect small quantities of material, for example for medical uses, to detect disease [94, 95], illicit-or-otherwise drugs [96, 97] or explosives 98 100]. For this purpose, it is desirable to maximise the average enhancement factor, $\langle\mathrm{EF}\rangle$. The other aim is to detect single molecules. The discussion of this is more complicated. This requires both that the signal from the single molecule is large enough to be detected, and also that the signal from any other molecules is not so large as to interfere with the signal from the first molecule. It has been estimated that enhancement factors of only $10^{4}$ are sufficient to observe single molecules, so the lower limit of $\mathrm{EF}_{\text {max }}$ is $10^{4}$. Regarding additional molecules providing signal, there are two main approaches to combat this. The first is to reduce the concentration of analytes, as was used in the first reports of single-molecule SERS[24, 25]. In order to counter the low number of events that low concentrations lead to, it may be combined with the bianalyte method[26] in order to distinguish statistically single- and multi-molecule events, allowing higher concentrations to be used. The other possible means of increasing the number of single-molecule events is to use a substrate such that the metric $R$ is large. This means that high-enhancement events, while perhaps rare, are very strong compared to average events. This entails choosing a substrate with strong localisation, such that when a molecule is at a hot-spot, with large enhancement, the signals from other molecules are much smaller. This approach may also be combined with the bianalyte method, in order to allow higher concentrations of analytes to be used while still distinguishing single-molecule events. This study will be a useful guide in this context. 



\section{Discussion}

The results in this thesis are based in two separate areas, unified by the field of light scattering. The first area relates to improving the extended boundary-condition method for fast and accurate modelling of how light scatters by particles. The second area focuses on the behaviour of the near-field of light in the region near to a metallic particle in the context of plasmonics and SERS.

Regarding the EBCM, the most general results presented in Chapter 4 relate to the forms of the integrals used to calculate the $T$-matrix. These new expressions are advantageous both because they are analytically simpler, permitting manipulation for special cases, and they also exhibit fewer numerical problems in some cases, by analytically simplifying problematic terms. These expressions form the basis of all of the other work on the EBCM in this thesis.

Following the presentation of these forms they were used, also in Chapter 4, to explore some linear relations between different integrals. General relations between 12 elements from $\mathbf{L}^{1}, \mathbf{L}^{2}, \mathbf{K}^{1}$ and $\mathbf{K}^{2}$ were found, and an expression for these derived. These results, as they stand, do not allow a more efficient means of calculating the matrices yet, but it is hoped that future work in this area will extend the relations to different $m$-values, with the possibility of reducing the computational cost of the method.

Both of the preceding contributions are general, and apply to all axisymmetric particles. The next results are shape-specific, and greatly improve the ability of the EBCM to model spheroids. In Chapter 5, the cause of severe numerical problems in the calculation of the $T$-matrix was examined. Firstly, the form of the problem was confirmed. This is, for the special cases of spheroids and offset spheres, a problem in the calculation of the matrix $\mathbf{Q}$. Other shapes suffer from numerical problems in the 


\section{Discussion}

EBCM, but the cause of these, while not studied here, is generally assumed to be more closely related to matrix inversion. However, spheroids and offset spheres have poorly-behaved integrals, where dominant terms in the power series of the integrands should integrate to zero, but numerical errors due to insufficient precision lead to catastrophic results. Further to this, it is possible to reformulate the integrals such that these problematic terms are removed, which is the subject of the second part of Chapter 5. Here, the behaviour of a series method to obtain the integrands is explored, and coupled with a recursion approach to populate all of the required entries. Using this method, it is now possible to obtain the correct $Q$-matrix, which may be easily inverted to obtain $\mathbf{T}$.

It is hoped that this method will allow the investigation of the behaviour of the $T$-matrix method close to the surface of spheroids, as well as the behaviour of the convergence of $T$-matrix entries under inversion. It permits the easy treatment of spheroids, approaching the ease of use as for spheres under Mie theory. The different behaviour of spheroid and offset spheres compared to other shapes means that these shapes are not in general useful to benchmark the performance of code. The steps that cause problems for other shapes (mainly inversion) are not problematic for spheroids and spheres, while the steps that cause problems for spheroids and spheres (integration) is not such a concern in general.

The second main area of this thesis is the subject of Chapter 6. This begins by using COMSOL finite-element modelling to replicate some experimental nanostructures, to better understand the surface-enhanced spectroscopic signals from experiments. This provided support to different experimental studies. In the bipyramid case, the simulations agree with the assumption that the CTAB bilayer prevents adsorption, as the metric $R$ over the entire particle surface as calculated does not match the experimental estimate, while when the area over which it is calculated is reduced, the agreement is much better.

In the study involving chains of spheres, the observed saturation in the shift of the resonance as silver spheres aggregate was modelled successfully. The simplest case of a dimer was modelled, and the results from both generalised Mie theory and COMSOL were compared, and yielded excellent agreement, supporting the use of 
COMSOL for the longer chains.

The last part of Chapter 6 concerns the distribution of the SERS enhancement on the surface of resonant nanoparticles. Here a selection of metrics which measure the degree to which hot-spots are localised, independent of the magnitude of the enhancement are presented. These are relevant especially when trying to optimise substrates for particular tasks, usually either maximising total signal, or for singlemolecule detection, highlighting one source of signal compared to others. The use of shapes which permit modifying the global aspect ratio and local curvature independently allows us to examine the contributions these different geometrical parameters have on the localisation. The results confirm what is intuitively suspected, that it is the local curvature that defines the hot-spot characteristics, while the overall shape has more of an effect on the frequency of the resonance.

One of the outcomes of this study into localisation was to demonstrate that by changing both the overall shape, and the curvature at the tips of a particle, it is possible to obtain normalised extinction behaviour that is very similar, for a collection of shapes that have only slight geometric differences. However, these shapes, which would be very difficult to distinguish in the far-field, have very different near-field behaviour. This should encourage caution especially when attempting to deduce exact local shape by matching far-field properties, and then draw conclusions about the near-field.

Using this knowledge, it is hoped that more efficient SERS substrates might be sought, that better suit the problem at hand. Also, further study into the effect of local shape on near-field behaviour, that might ameliorate some of the concerns above, could use these results as a starting point.

The initial aim of this project was to improve the applicability of the $T$-matrix method in the area of plasmonics, to make it a compelling tool for the study plasmonic systems. As it progressed, we instead made more gains on fundamental aspects of the EBCM and $T$-matrix method, which have wider applicability than in plasmonics. The progress made for spheroids has made the $T$-matrix an appropriate method to use for plasmonics applications involving spheroids. For other shapes, the progress is still to be made, and hence we used COMSOL to solve problems 


\section{Discussion}

involving other geometries. 


\section{A Bessel Function Series Expressions}

The Riccati-Bessel functions may be written in a power series as ([50], Sections 9.1.2 and 9.1.10)

$$
\begin{aligned}
& \chi_{n}(x)=\frac{-1}{x^{n}} \sum_{i=0}^{\infty} \frac{\left(-\frac{1}{2}\right)^{i}}{i !} a_{i, n} x^{2 i} \\
& \psi_{k}(s x)=(s x)^{k+1} \sum_{i=0}^{\infty} \frac{\left(-\frac{1}{2}\right)^{i}}{i !} b_{i, k} x^{2 i} s^{2 i}
\end{aligned}
$$

where the coefficients $a$ and $b$ are defined as

$$
\begin{aligned}
a_{i, n} & =(2 n-1) ! ! \prod_{\substack{j=1 \\
\text { odd }}}^{2 i-1} \frac{1}{j-2 n} \\
& = \begin{cases}(-1)^{i}(2 n-2 i-1) ! ! & \text { for } i \leq n \\
\frac{(-1)^{n}}{(2 i-1-2 n) ! !} & \text { for } i>n\end{cases} \\
b_{i, k} & =\frac{1}{(2 k+1) ! !} \prod_{j=1}^{2 i-1} \frac{1}{2 k+j+2} \\
& =\frac{1}{(2 k+2 i+1) ! !}
\end{aligned}
$$

where $(2 l+1)$ !! is the double factorial operation,

$$
(2 l+1) ! !=1 \times 3 \times 5 \times \cdots \times(2 l+1)=\frac{(2 l+1) !}{2^{l} l !} .
$$




\section{A Bessel Function Series Expressions}

We are also able to express the derivatives of these functions as

$$
\begin{gathered}
\chi_{n}^{\prime}(x)=\left.\frac{\mathrm{d}}{\mathrm{d} z} \chi_{n}(z)\right|_{z=x}=\frac{-1}{x^{n+1}} \sum_{i=0}^{\infty} \frac{\left(-\frac{1}{2}\right)^{i}(2 i-n)}{i !} a_{i, n} x^{2 i} \\
\psi_{k}^{\prime}(s x)=\left.\frac{\mathrm{d}}{\mathrm{d} z} \psi_{k}(z)\right|_{z=s x}=(s x)^{k} \sum_{i=0}^{\infty} \frac{\left(-\frac{1}{2}\right)^{i}(2 i+k+1)}{i !} b_{i, k} x^{2 i} s^{2 i} .
\end{gathered}
$$

The Riccati-Bessel functions are related to the spherical Bessel functions by

$$
\begin{aligned}
\psi_{n}(x) & =x j_{n}(x) \\
\chi_{n}(x) & =x y_{n}(x) \\
\xi_{n}(x) & =x h_{n}^{(1)}(x),
\end{aligned}
$$

and the spherical Bessel functions are related to the Bessel functions as

$$
\begin{aligned}
j_{n}(x) & =\sqrt{\frac{\pi}{2 x}} J_{n+1 / 2}(x) \\
y_{n}(x) & =\sqrt{\frac{\pi}{2 x}} Y_{n+1 / 2}(x) \\
h_{n}^{(1)}(x) & =\sqrt{\frac{\pi}{2 x}} H_{n+1 / 2}^{(1)}(x) .
\end{aligned}
$$




\section{B Angular Function Definitions and Relations}

There are a variety of functions that depend on the angle $\theta$ that we use. These functions are all based on the associated Legendre functions. This appendix contains first the definitions of the various functions used, and following that are various relations that are used in this thesis. The relations are broken down into two groups. The first considers relations that are used in order to numerically compute the functions, while the second considers analytic relations between the functions that are used in various proofs.

\section{B.1 Function Definitions}

The function which underlies all of the others is the associated Legendre function. This is a solution to the differential equation (as from [50], Eq. (8.1.1))

$$
\left(1-x^{2}\right) \frac{\mathrm{d}^{2} w}{\mathrm{~d} x^{2}}-2 x \frac{\mathrm{d} w}{\mathrm{~d} x}+\left[n(n+1)-\frac{m^{2}}{1-x^{2}}\right] w=0
$$

where we are interested in solutions

$$
w=P_{n}^{m}(\cos \theta)
$$

where $m$ is the order of the solutions, $n$ is the degree, and we use $x=\cos \theta$. Cases where $m=0$ are known as Legendre polynomials, $P_{n}(x)$. The associated Legendre 
functions may be written in terms of the polynomial as (for $m \geq 0$ )

$$
P_{n}^{m}(x)=(-1)^{m}\left(1-x^{2}\right)^{m / 2} \frac{\mathrm{d}^{m}}{\mathrm{~d} x^{m}} P_{n}(x)
$$

where the polynomial is given by the expression

$$
P_{n}(x)=\frac{1}{2^{n} n !} \frac{\mathrm{d}^{n}}{\mathrm{~d} x^{n}}\left(x^{2}-1\right)^{n}
$$

The factor $(-1)^{m}$ in the definition of Eq. (B.3) is known as the Condon-Shortley phase. In the case of negative $m$, the expression for the associated Legendre function is

$$
P_{n}^{-m}(x)=(-1)^{m} \frac{(n-m) !}{(n+m) !} P_{n}^{m}(x)
$$

We do not use the associated Legendre functions directly, but rather some functions obtained from them, which have more favourable numerical properties [9]. We use a special case of the Wigner $d$-functions,

$$
d_{n m}(\theta)=d_{0 m}^{n}(\theta)=(-1)^{m} \sqrt{\frac{(n-m) !}{(n+m) !}} P_{n}^{m}(\cos \theta)
$$

where we make use of the simpler $d_{n m}(\theta)$ notation in this thesis. We also make use of the functions $\pi_{n m}(\theta)$ and $\tau_{n m}(\theta)$, defined in terms of $d_{n m}(\theta)$ as

$$
\begin{aligned}
\pi_{n m}(\theta) & =\frac{m d_{n m}(\theta)}{\sin \theta} \\
\tau_{n m}(\theta) & =\frac{\mathrm{d}}{\mathrm{d} \theta} d_{n m}(\theta) .
\end{aligned}
$$




\section{B.2 Numerical Computation of the Functions}

The function $\pi_{n m}(\theta)$ may be generated using a recursion relation, as [9]

$$
\pi_{n m}(\theta)=\frac{1}{\sqrt{n^{2}-m^{2}}}\left((2 n-1) \cos \theta \pi_{n-1, m}(\theta)\right)-\sqrt{(n-1)^{2}-m^{2}} \pi_{n-2, m}(\theta),
$$

with the initial conditions

$$
\begin{aligned}
\pi_{m, m-1}(\theta) & =0 \\
\pi_{m m}(\theta) & =m A_{m} \sin ^{m-1} \theta
\end{aligned}
$$

with $A_{m}$ defined recursively as

$$
\begin{aligned}
A_{0} & =1 \\
A_{m+1} & =A_{m} \sqrt{\frac{2 m+1}{2(m+1)}} .
\end{aligned}
$$

From the values $\pi_{n m}$, it is possible to calculate the $\tau_{n m}$ as

$$
\tau_{n m}(\theta)=\frac{-1}{m} \sqrt{n^{2}-m^{2}} \pi_{n-1, m}(\theta)+\frac{n}{m} \cos \theta \pi_{n m}(\theta),
$$

or making use of $d_{n m}$ instead of $\pi_{n m}$,

$$
\sin \theta \tau_{n m}(\theta)=-\sqrt{n^{2}-m^{2}} d_{n-1, m}(\theta)+n \cos \theta d_{n m}(\theta) .
$$

It is also possible to calculate the values for negative $m$ using simple relations, as

$$
\begin{aligned}
& \pi_{n,-m}(\theta)=(-1)^{m+1} \pi_{n m}(\theta) \\
& \tau_{n,-m}(\theta)=(-1)^{m} \tau_{n m}(\theta) .
\end{aligned}
$$

These schemes for calculating these functions are numerically stable and efficient [9]. In some cases, we use the function $d_{n m}$ rather than $\pi_{n m}$, but these are calculated from $\pi_{n m}$ (when $m \neq 0$ ). In the case where $m=0$, then $\pi_{n m}$ is zero, but $d_{n m}$ is 
not in general zero. Rather, in that case, the Legendre polynomial itself is used to obtain $d_{n, m=0}(\theta)=P_{n}(\cos \theta)$.

\section{B.3 Relations Used in Proofs}

For the angular functions, we have

$$
\left[\pi_{n} \tau_{k}+\tau_{n} \pi_{k}\right] \sin \theta=m\left(d_{n} d_{k}\right)^{\prime}
$$

which may be obtained by noting that $\frac{\mathrm{d}}{\mathrm{d} \theta} d_{n}=\tau_{n}$, and that $d_{n}=\pi_{n} \frac{\sin \theta}{m}($ for $m \neq 0$ ). This then follows from application of the chain rule.

We have the simple relations

$$
\frac{\mathrm{d}}{\mathrm{d} \theta}\left(d_{n} \sin \theta\right)=\tau_{n} \sin \theta+d_{n} \cos \theta
$$

and

$$
\frac{\mathrm{d}}{\mathrm{d} \theta}\left(\pi_{n} \sin \theta\right)=m \tau_{n}
$$

We also have the relations

$$
\begin{aligned}
{\left[\pi_{n} \pi_{k}+\tau_{n} \tau_{k}\right] \sin \theta } & =\left(d_{n} \tau_{k} \sin \theta\right)^{\prime}+k(k+1) d_{n} d_{k} \sin \theta \\
& =\left(\tau_{n} d_{k} \sin \theta\right)^{\prime}+n(n+1) d_{n} d_{k} \sin \theta
\end{aligned}
$$

This may be shown by considering the definition of $d_{n}(\theta)$ in terms of associated Legendre functions. We then have (using complete notation)

$$
\begin{aligned}
\tau_{n m}(\theta)=\frac{\mathrm{d}}{\mathrm{d} \theta} d_{0 m}^{n} & =-\sin \theta C_{n m} \frac{\mathrm{d}}{\mathrm{d}(\cos \theta)} P_{n}^{m}(\cos \theta) \\
& =-\sin \theta C_{n m}\left(P_{n}^{m}(\cos \theta)\right)^{\prime}
\end{aligned}
$$


where

$$
C_{n m}=(-1)^{m} \sqrt{\frac{(n-m) !}{(n+m) !}}
$$

and hence

$$
\begin{aligned}
\frac{\mathrm{d}}{\mathrm{d} \theta} \tau_{n m}(\theta) & =\frac{\mathrm{d}^{2}}{\mathrm{~d} \theta^{2}} d_{0 m}^{n}(\theta) \\
& =-\cos \theta C_{n m}\left(P_{n}^{m}(\cos \theta)\right)^{\prime}+\sin ^{2} \theta C_{n m}\left(P_{n}^{m}(\cos \theta)\right)^{\prime \prime}
\end{aligned}
$$

By making use of Eq. B.3 which defines the associated Legendre functions, we have

$$
\sin ^{2} \theta\left(P_{n}^{m}(\cos \theta)\right)^{\prime \prime}=2 \cos \theta\left(P_{n}^{m}(\cos \theta)\right)^{\prime}-\left[n(n+1)-\frac{m^{2}}{\sin ^{2} \theta}\right] P_{n}^{m}(\cos \theta)
$$

and thus

$$
\frac{\mathrm{d}}{\mathrm{d} \theta} \tau_{n m}(\theta)=\cos \theta C_{n m}\left(P_{n}^{m}(\cos \theta)\right)^{\prime}-C_{n m}\left[n(n+1)-\frac{m^{2}}{\sin ^{2} \theta}\right] P_{n}^{m}(\cos \theta)
$$

From this, we can calculate

$$
\begin{aligned}
\frac{\mathrm{d}}{\mathrm{d} \theta}\left(\tau_{n m}(\theta) \sin \theta\right) & =-\sin \theta C_{n m}\left[n(n+1)-\frac{m^{2}}{\sin ^{2} \theta}\right] P_{n}^{m}(\cos \theta) \\
& =\frac{m^{2}}{\sin \theta} d_{0 m}^{n}(\theta)-n(n+1) \sin \theta d_{0 m}^{n}(\theta) \\
& =m \pi_{n}-n(n+1) d_{0 m}^{n} \sin \theta
\end{aligned}
$$

and then

$$
\begin{aligned}
\left.\frac{\mathrm{d}}{\mathrm{d} \theta}\left(\tau_{n m}(\theta) d_{0 m}^{k}(\theta) \sin \theta\right)\right)= & \sin \theta\left(\tau_{n m}(\theta) \tau_{k m}(\theta)+\pi_{n m}(\theta) \pi_{k m}(\theta)\right) \\
& \left.-n(n+1) d_{0 m}^{n}(\theta) d_{0 m}^{k}(\theta)\right)
\end{aligned}
$$

This may be written in slightly different notation, by omitting the argument $\theta$ of 
B Angular Function Definitions and Relations

functions, and also omitting the index $m$, as

$$
\left(\tau_{n} d_{k} \sin \theta\right)^{\prime}=\sin \theta\left(\tau_{n} \tau_{k}+\pi_{n} \pi_{k}-n(n+1) d_{n} d_{k}\right)
$$

By exchange of indices, we also trivially arrive at

$$
\left(d_{n} \tau_{k} \sin \theta\right)^{\prime}=\sin \theta\left(\tau_{n} \tau_{k}+\pi_{n} \pi_{k}-k(k+1) d_{n} d_{k}\right)
$$




\section{Vector Spherical Wavefunctions}

Vector spherical wavefunctions (VSWFs) are central to both Mie theory and the $T$-matrix method, as the fields are expressed in terms of series of these functions. In this appendix, the definitions of the relevant wavefunctions are provided for completeness. This follows Appendix C of Ref. 9].

The function $\vec{M}$ is defined as

$$
\vec{M}(k r, \theta, \phi)=\nabla \times(\vec{r} f(k r, \theta, \phi))
$$

and $\vec{N}$ is defined from this as

$$
\vec{N}=\frac{1}{k} \nabla \times \vec{M}
$$

Obviously, the definition of $f$ is crucial to these functions. In these, $k$ is the wavenumber in the appropriate medium, and not necessarily the incident wavenumber. The form of $f$ is

$$
f_{n m}(x, \theta, \phi)=\gamma_{n m} z_{n}(x) \sqrt{\frac{(n+m) !}{(n-m) !}} d_{n m}(\theta) e^{i m \phi}
$$

where $z_{n}$ is one of the spherical Bessel- or Hankel-functions, as necessary to be regular at the origin $\left(j_{n}(x)\right)$ or behave as outgoing spherical waves $\left(h_{n}^{(1)}(x)\right)$, and $x=k r$. Regular solutions, $\vec{M}^{(1)}$ and $\vec{N}^{(1)}$, make use of $j_{n}$, while the irregular solutions $\vec{M}^{(3)}$ and $\vec{N}^{(3)}$ contain $h_{n}^{(1)}$. We also make use of

$$
\gamma_{n m}=\sqrt{\frac{(2 n+1)(n-m) !}{4 \pi n(n+1)(n+m) !}} .
$$




\section{Vector Spherical Wavefunctions}

This expression is normalised so that the integration over angle of two such functions is normalised to one. Other normalisation schemes are possible.

It is possible to express the wavefunctions as

$$
\begin{aligned}
\vec{M} & =\gamma_{n m} z_{n}(x) \vec{C}_{n m}(\theta, \phi) \\
\vec{N} & =\gamma_{n m}\left(\frac{n(n+1)}{x} z_{n}(x) \vec{P}_{n m}(\theta, \phi)+\frac{1}{x} z_{n}^{\prime}(x) \vec{B}_{n m}(\theta, \phi)\right)
\end{aligned}
$$

where we use the vectors

$$
\begin{aligned}
& \vec{B}_{n m}(\theta, \phi)=r \nabla\left(P_{n}^{m}(\cos \theta) e^{i m \phi}\right) \\
& =\left(\hat{\theta} \frac{\mathrm{d}}{\mathrm{d} \theta} P_{n}^{m}(\cos \theta)+\hat{\phi} \frac{i m}{\sin \theta} P_{n}^{m}(\cos \theta)\right) e^{i m \phi} \\
& =(-1)^{m} \sqrt{\frac{(n+m) !}{(n-m) !}} \vec{B}_{n m}(\theta) e^{i m \phi} \\
& \vec{C}_{n m}(\theta, \phi)=\nabla \times\left(\vec{r} P_{n}^{m}(\cos \theta) e^{i m \phi}\right) \\
& =\left(\hat{\theta} \frac{i m}{\sin \theta} P_{n}^{m}(\cos \theta)-\hat{\phi} \frac{\mathrm{d}}{\mathrm{d} \theta} P_{n}^{m}(\cos \theta)\right) e^{i m \phi} \\
& =(-1)^{m} \sqrt{\frac{(n+m) !}{(n-m) !}} \vec{C}_{n m}(\theta) e^{i m \phi} \\
& =\vec{B}_{n m}(\theta, \phi) \times \hat{r} \\
& \vec{P}_{n m}(\theta, \phi)=\hat{r} P_{n}^{m}(\cos \theta) e^{i m \phi} \\
& =(-1)^{m} \sqrt{\frac{(n+m) !}{(n-m) !}} \vec{P}_{n m}(\theta) e^{i m \phi} .
\end{aligned}
$$

In these expressions, we use the $\phi$-independent vectors

$$
\begin{aligned}
\vec{B}_{n m}(\theta) & =\hat{\theta} \tau_{n m}(\theta)+\hat{\phi} i \pi_{n m}(\theta) \\
\vec{C}_{n m}(\theta) & =\hat{\theta} i \pi_{n m}(\theta)-\hat{\phi} \tau_{n m}(\theta) \\
\vec{P}_{n m}(\theta) & =\hat{r} d_{n m}(\theta) .
\end{aligned}
$$




\section{D $J$-integral Expressions}

In this appendix, the forms of the $J$-integrals for axisymmetric scatterers, as given in Ref. [9], are provided. In this case, the integrals are zero unless the indices $m$ and $m^{\prime}$ are equal, and hence the index $m^{\prime}$ has been dropped.

The elements of $J$ are given by

$J_{m n n^{\prime}}^{11}=-\frac{i}{2} a_{n} a_{n^{\prime}} \int_{-1}^{+1} \mathrm{~d}(\cos \theta) r^{2} h_{n}^{(1)}\left(k_{1} r\right) j_{n^{\prime}}\left(k_{2} r\right)\left[\pi_{m n}(\theta) \tau_{m n^{\prime}}(\theta)+\tau_{m n}(\theta) \pi_{m n^{\prime}}(\theta)\right]$

$$
\begin{aligned}
J_{m n n^{\prime}}^{12}= & \frac{1}{2} a_{n} a_{n^{\prime}} \int_{-1}^{+1} \mathrm{~d}(\cos \theta) r^{2} j_{n^{\prime}}\left(k_{2} r\right)\left\{\frac{1}{k_{1} r} \frac{\mathrm{d}}{\mathrm{d}\left(k_{1} r\right)}\left[k_{1} r h_{n}^{(1)}\left(k_{1} r\right)\right]\right. \\
& \left.\times\left[\pi_{m n}(\theta) \pi_{m n^{\prime}}(\theta)+\tau_{m n}(\theta) \tau_{m n^{\prime}}(\theta)\right]+\frac{r_{\theta}}{r} n(n+1) \frac{h_{n}^{(1)}\left(k_{1} r\right)}{k_{1} r} d_{0 m}^{n}(\theta) \tau_{m n^{\prime}}(\theta)\right\} \\
J_{m n n^{\prime}}^{21}= & -\frac{1}{2} a_{n} a_{n^{\prime}} \int_{-1}^{+1} \mathrm{~d}(\cos \theta) r^{2} h_{n}^{(1)}\left(k_{1} r\right)\left\{\frac{1}{k_{2} r} \frac{\mathrm{d}}{\mathrm{d}\left(k_{2} r\right)}\left[k_{2} r j_{n^{\prime}}\left(k_{2} r\right)\right]\right. \\
& \left.\times\left[\pi_{m n}(\theta) \pi_{m n^{\prime}}(\theta)+\tau_{m n}(\theta) \tau_{m n^{\prime}}(\theta)\right]+\frac{r_{\theta}}{r} n^{\prime}\left(n^{\prime}+1\right) \frac{j_{n^{\prime}}\left(k_{2} r\right)}{k_{2} r} \tau_{m n}(\theta) d_{0 m}^{n^{\prime}}(\theta)\right\}
\end{aligned}
$$




\section{$D J$-integral Expressions}

$$
\begin{aligned}
J_{m n n^{\prime}}^{22}= & -\frac{i}{2} a_{n} a_{n^{\prime}} \int_{-1}^{+1} \mathrm{~d}(\cos \theta) r^{2}\left(\frac{1}{k_{1} r} \frac{\mathrm{d}}{\mathrm{d}\left(k_{1} r\right)}\left[k_{1} r h_{n}^{(1)}\left(k_{1} r\right)\right] \frac{1}{k_{2} r} \frac{\mathrm{d}}{\mathrm{d}\left(k_{2} r\right)}\left[k_{2} r j_{n^{\prime}}\left(k_{2} r\right)\right]\right. \\
& \times\left[\pi_{m n}(\theta) \tau_{m n^{\prime}}(\theta)+\tau_{m n}(\theta) \pi_{m n^{\prime}}(\theta)\right] \\
& +\frac{r_{\theta}}{r}\left\{n(n+1) \frac{h_{n}^{(1)}\left(k_{1} r\right)}{k_{1} r} \frac{1}{k_{2} r} \frac{\mathrm{d}}{\mathrm{d}\left(k_{2} r\right)}\left[k_{2} r j_{n^{\prime}}\left(k_{2} r\right)\right]\right. \\
+ & \left.\left.\frac{1}{k_{1} r} \frac{\mathrm{d}}{\mathrm{d}\left(k_{1} r\right)}\left[k_{1} r h_{n}^{(1)}\left(k_{1} r\right)\right] n^{\prime}\left(n^{\prime}+1\right) \frac{j_{n^{\prime}}\left(k_{2} r\right)}{k_{2} r}\right\} \pi_{m n}(\theta) d_{0 m}^{n^{\prime}}(\theta)\right),
\end{aligned}
$$

where $a_{n}=\sqrt{\frac{2 n+1}{n(n+1)}}$, and $\pi, \tau$ are as defined in Section B.1. To simplify the expressions, a different notation is used. This does not alter the expressions, but simply uses a slightly different notation, introduced in Ref. [1], as

$$
\begin{aligned}
& J_{n k}^{11}=-\frac{i a_{n} a_{k}}{s k_{1}^{2}} \int_{0}^{\pi} \mathrm{d} \theta \sin \theta \xi_{n} \psi_{k}\left[\pi_{n} \tau_{k}+\tau_{n} \pi_{k}\right] \\
& J_{n k}^{12}=\frac{a_{n} a_{k}}{s k_{1}^{2}} \int_{0}^{\pi} \mathrm{d} \theta \sin \theta \psi_{k}\left\{\xi_{n}^{\prime}\left[\pi_{n} \pi_{k}+\tau_{n} \tau_{k}\right]+\frac{x_{\theta}}{x^{2}} n(n+1) \xi_{n} d_{n} \tau_{k}\right\} \\
& J_{n k}^{21}=-\frac{a_{n} a_{k}}{s k_{1}^{2}} \int_{0}^{\pi} \mathrm{d} \theta \sin \theta \xi_{n}\left\{\psi_{k}^{\prime}\left[\pi_{n} \pi_{k}+\tau_{n} \tau_{k}\right]+\frac{x_{\theta}}{s x^{2}} k(k+1) \psi_{k} \tau_{n} d_{k}\right\} \\
& J_{n k}^{22}=-\frac{i a_{n} a_{k}}{s k_{1}^{2}} \int_{0}^{\pi} \mathrm{d} \theta \sin \theta\left\{\xi_{n}^{\prime} \psi_{k}^{\prime}\left[\pi_{n} \tau_{k}+\tau_{n} \pi_{k}\right]+\left[n(n+1) \xi_{n} \psi_{k}^{\prime}+\frac{k(k+1)}{s} \xi_{n}^{\prime} \psi_{k}\right] \frac{x_{\theta}}{x^{2}} \pi_{n} d_{k}\right\},
\end{aligned}
$$

where this notation has an implied argument of $\theta$ for $\pi, d, x$ and $\tau, s=k_{2} / k_{1}, \xi_{n}$ has an argument of $x(\theta)=k_{1} r(\theta), \psi_{k}$ has an argument of $s x$, and the index $m$ is implied for all the $J$-matrices, as well as for $\pi, d$ and $\tau$. The Riccati-Bessel functions $\xi$ and $\psi$ are given by $\xi_{n}(x)=x h_{n}^{(1)}(x)$ and $\psi_{k}(x)=x j_{k}(x)$, and are described in more detail in Appendix A. We also use the index $k$ instead of $n^{\prime}$. 


\section{E Proof that Leading Terms Make No Contribution in Offset-Sphere Integrals}

Here is presented a proof that certain leading terms in the integrals for $\mathbf{U}$ integrate to zero in the case of an offset sphere. These proofs largely follow those of the spheroid (as in Section 5.4), but the details do differ significantly, for two reasons. Firstly, because of the reduction in symmetry, every entry in the matrix is now nonzero, not only half of the entries as for spheroids. The other difference is that the specific relations on the geometry of the spheroid are no longer valid. Instead, we must use different relations applicable to the offset sphere.

These proofs follow the same basic structure as those presented in Ref. [3], though the proofs in this thesis cover all cases (both parities of $n+k$, as well as all of the integrands required).

\section{E.1 Some Definitions}

We here define some terms relating to the geometry. As with the spheroid, these geometric relations are key to the special behaviour of the integrals for this shape. For the offset sphere, the geometry is as defined in Section 5.1 .

In order to exploit parity properties, that is, the transformation $\theta \rightarrow \pi-\theta$, we 
E Proof that Leading Terms Make No Contribution in Offset-Sphere Integrals

write

$$
\begin{aligned}
& x(\theta)=k_{1} r(\theta)=f(\theta)+g(\theta) \\
& f(\theta)=-k_{1} D \cos \theta \equiv f \\
& g(\theta)=k_{1} \sqrt{a^{2}-D^{2} \sin ^{2} \theta} \equiv g .
\end{aligned}
$$

Looking at the derivatives of these, we have also

$$
\begin{aligned}
& x_{\theta}=f_{\theta}+g_{\theta} \\
& f_{\theta}=k_{1} D \sin \theta \\
& g_{\theta}=-k_{1} D^{2} \sin \theta \cos \theta / g,
\end{aligned}
$$

where these functions have the parity properties

$$
\begin{aligned}
f(\pi-\theta) & =-f(\theta) \\
f_{\theta}(\pi-\theta) & =f_{\theta}(\theta) \\
g(\pi-\theta) & =g(\theta) \\
g_{\theta}(\pi-\theta) & =-g_{\theta}(\theta) .
\end{aligned}
$$

It is also worth noting the relations

$$
\begin{aligned}
f_{\theta} f & =g_{\theta} g \\
g^{2}-f^{2} & =\frac{1}{a^{2}-D^{2}} \equiv b \\
\frac{1}{f+g} & =\frac{g-f}{b} \\
\frac{x_{\theta}}{x} & =\frac{f_{\theta}}{g}=\frac{g_{\theta}}{f},
\end{aligned}
$$

and some parity properties of the angular functions, namely

$$
\begin{aligned}
d_{n}(\pi-\theta) d_{k}(\pi-\theta) & =(-1)^{n+k} d_{n}(\theta) d_{k}(\theta) \\
\sin (\pi-\theta) \tau_{n}(\pi-\theta) d_{k}(\pi-\theta) & =(-1)^{n+k} \sin \theta \tau_{n}(\theta) d_{k}(\theta) .
\end{aligned}
$$


We also define two operators that allow us to decompose functions into parts of 'even' or 'odd' parity (when using the transformation $\theta \rightarrow \pi-\theta$ ). Thus, we define, for some arbitrary function $h(\theta)$

$$
h(\theta)=\mathcal{E}[h](\theta)+\mathcal{U}[h](\theta)
$$

with $\mathcal{E}$ the 'even' part, and $\mathcal{U}$ the 'odd' part, such that

$$
\begin{aligned}
\mathcal{E}[h](\pi-\theta) & =\mathcal{E}[h](\theta) \\
\mathcal{U}[h](\pi-\theta) & =-\mathcal{U}[h](\theta),
\end{aligned}
$$

and which display the properties (for an arbitrary function $j(\theta)$ )

$$
\begin{aligned}
\mathcal{E}[h j] & =\mathcal{E}[h] \mathcal{E}[j]+\mathcal{U}[h] \mathcal{U}[j] \\
\mathcal{U}[h j] & =\mathcal{E}[h] \mathcal{U}[j]+\mathcal{U}[h] \mathcal{E}[j]
\end{aligned}
$$

Further, we define, for the case of $p$ an integer,

$$
\begin{aligned}
e_{p} & =\mathcal{E}\left[x_{\theta} x^{p}\right] \\
u_{p} & =\mathcal{U}\left[x_{\theta} x^{p}\right] \\
\tilde{e}_{p} & =\mathcal{E}\left[x^{p}\right] \\
\tilde{u}_{p} & =\mathcal{U}\left[x^{p}\right] .
\end{aligned}
$$

We may, using the properties of $x$, also deduce that

$$
\begin{aligned}
\mathcal{E}\left[x_{\theta} x^{p+2}\right] & =\mathcal{E}\left[x_{\theta} x^{p}\right]\left(f^{2}+g^{2}\right)+2 f g \mathcal{U}\left[x_{\theta} x^{p}\right] \\
\mathcal{U}\left[x_{\theta} x^{p+1}\right] & =f \mathcal{E}\left[x_{\theta} x^{p}\right]+g \mathcal{U}\left[x_{\theta} x^{p}\right] \\
\mathcal{E}\left[x_{\theta} x^{p+1}\right] & =g \mathcal{E}\left[x_{\theta} x^{p}\right]+f \mathcal{E}\left[x_{\theta} x^{p}\right] \\
\mathcal{U}\left[x^{\theta} x^{p+2}\right] & =2 f g \mathcal{E}\left[x_{\theta} x^{p}\right]+\mathcal{U}\left[x_{\theta} x^{p}\right]\left(f^{2}+g^{2}\right)
\end{aligned}
$$

and thus by combining Eqs. (E.26) and (E.27) we have

$$
e_{p+2}=b e_{p}+2 f u_{p+1}
$$


E Proof that Leading Terms Make No Contribution in Offset-Sphere Integrals

and by combining Eqs. (E.28) and $(\mathrm{E.29}$ ) and re-numbering we have

$$
u_{p+1}=b u_{p-1}+2 f e_{p}
$$

where $b=g^{2}-f^{2}$ is as defined in Eq. E.12). These two relations are also valid for $\tilde{e}_{p}$ and $\tilde{u}_{p}$.

We are able to write explicitly the $\theta$-dependence of the first terms

$$
\begin{aligned}
& e_{0}=f_{\theta} \propto \sin \theta \\
& u_{1}=2 f_{\theta} f \propto \sin \theta \cos \theta
\end{aligned}
$$

and using recursion, these functions are expressable as (for $q \geq 0$ )

$$
\begin{aligned}
e_{2 q} & =\sin \theta \mathcal{P}_{2 q}(\cos \theta) \\
u_{2 q+1} & =\sin \theta \mathcal{P}_{2 q+1}(\cos \theta)
\end{aligned}
$$

where $\mathcal{P}_{N}(\cos \theta)$ represents a polynomial in $\cos \theta$ of degree $N$ or less, as was used in Section 5.4 for the case of spheroids.

For negative $q$, we have $u_{-1}=0$ as $x_{\theta} / x$ is even, and $e_{-2}=f_{\theta} / b \propto \sin \theta$. Thus (for $q \geq 1$ )

$$
\begin{array}{r}
e_{-2 q}=\sin \theta \mathcal{P}_{2 q-2}(\cos \theta) \\
u_{-(2 q+1)}=\sin \theta \mathcal{P}_{2 q-1}(\cos \theta) .
\end{array}
$$

It is also possible to obtain forms for $\tilde{e}_{p}$ and $\tilde{u}_{p}$. We start with

$$
\begin{aligned}
\tilde{e}_{0} & =1 \\
\tilde{u}_{1} & =f \propto \cos \theta \\
\tilde{u}_{-1} & =-f \propto \cos \theta .
\end{aligned}
$$


It is then possible to deduce that

$$
\begin{aligned}
\tilde{e}_{2 q} & =\mathcal{P}_{|2 q|}(\cos \theta) \\
\tilde{u}_{2 q+1} & =\mathcal{P}_{|2 q+1|}(\cos \theta),
\end{aligned}
$$

for all integer $q$.

Also, it is possible to show that

$$
\begin{aligned}
\sin \theta \tilde{e}_{p+1} & =\cos \theta u_{p}+e_{p+1} /\left(k_{1} D\right) \\
\sin \theta \tilde{u}_{p+1} & =\cos \theta e_{p}+u_{p+1} /\left(k_{1} D\right) .
\end{aligned}
$$

\section{E.2 Proofs}

Here are provided proofs that the terms we expect integrate to zero do integrate identically to zero.

\section{E.2.1 Integrals $K^{1}$ and $K^{2}$}

We begin by considering the integral $\mathbf{K}^{1}$. We make use of the expansion of the Bessel function product, as for the spheroid in Eq. (5.39). The key now is to expand $K_{n k p}^{1}$ into two parts, with different parities.

For $n+k$ even, we have

$$
K_{n k p}^{1}=\int_{0}^{\pi} \mathrm{d} \theta m d_{n} d_{k} e_{p} .
$$

Using Eq. (E.34) and Eq. 5.19, we can see that this integral is zero whenever $0 \leq p \leq n-k-1$ or, by using Eq. E.36) for negative $p$ we see that the integral is zero when $-p-2 \leq n-k-1$, thus for $k-n \leq p \leq n-k-2$ the integral is zero. This matches our expectations. 
For the odd values of $n+k$, then we also have $p$ odd by parity, and

$$
K_{n k p}^{1}=\int_{0}^{\pi} \mathrm{d} \theta m d_{n} d_{k} u_{p}
$$

For $p=-1$, then $u_{-1}=0$ and this is trivially zero. For $p \geq 0$, this integral is zero when $p \leq n-k-1$, and for $p \leq-3$, then the integral is zero for $-p-2 \leq n-k-1$, meaning that the integral is zero for $k-n \leq p \leq n-k-2$, which is the entire range that we expect.

The proof for $\mathbf{K}^{2}$ is identical to this, as $K_{n k p}^{1}$ has exactly the same form as $K_{n k p}^{2}$.

\section{E.2.2 Integrals $\mathrm{L}^{1}$ and $\mathrm{L}^{2}$}

As with the proof for the spheroid, we start by considering $L_{n k p}^{1}$, the terms in the expansion of the integral in powers of $x$. In this case, based on parity, when $n+k+p$ is even then the integral is zero.

For $n+k$ even, then we want to examine the case where $p$ is odd. By parity, that means that

$$
L_{n k p}^{1}=\int_{0}^{\pi} \mathrm{d} \theta \sin \theta \tau_{n} d_{k} u_{p}
$$

From Eqs. E.35) and (E.37) we know that this integral is zero for $p \geq 0$ when $p \leq n-k-2$. For $p=-1$ then $u_{-1}=0$ and so the integral is zero. For $p \leq-3$ then by Eqs. (E.37) and (5.29) the integral is zero when $-p-2 \leq n-k-2$, meaning the integral is zero for $k+1-n \leq p \leq n-k-3$, which is the range that we require.

For $n+k$ odd, then $p$ is even. We than have

$$
L_{n k p}^{1}=\int_{0}^{\pi} \mathrm{d} \theta \sin \theta \tau_{n} d_{k} e_{p}
$$

For $p \geq 0$, the integral is zero for $p \leq n-k-2$. For $p$ negative, then the integral is zero when $-p-2 \leq n-k-2$. These combine so that the integral is zero whenever $k+1-n \leq p \leq n-k-3$. 
In the case of $\mathbf{L}^{2}$, the same logic holds, as the only difference from $\mathbf{L}^{1}$ is the indices of $d$ and $\tau$. As these only appear as $|n-k|$ in the working, these are interchangeable and the same proof holds for $\mathbf{L}^{2}$.

\section{E.2.3 Integrals $\mathrm{L}^{3}$ and $\mathrm{L}^{4}$}

We now look at the proof that $\mathbf{L}^{3}$ exhibits the same cancellation, following the same approach as for the spheroid case. The integral is

$$
L_{n k}^{3}=\int_{0}^{\pi} \mathrm{d} \theta \sin \theta d_{k} \psi_{k}^{\prime}\left[x_{\theta} \tau_{n} \chi_{n}^{\prime}-n(n+1) d_{n} \chi_{n}\right]
$$

and we may expand this as a Laurent series as

$$
\begin{aligned}
L_{n k}^{3} & =\sum_{q=0}^{\infty} L_{n k p}^{3}, \\
L_{n k p}^{3} & =\int_{0}^{\pi} \mathrm{d} \theta \sin \theta d_{k}\left[\alpha_{n k q}(s) x_{\theta} x^{p} \tau_{n}+\beta_{n k q}(s) x^{p+1} d_{n}\right]
\end{aligned}
$$

and we note that the two terms in $L_{n k p}^{3}$ scale the same, as $x_{\theta}$ scales as $x$. We may also write

$$
\begin{aligned}
& L_{n k p}^{3}=\alpha_{n k q}(s) L_{n k p}^{31}+\beta_{n k q}(s) L_{n k p}^{32} \\
& L_{n k p}^{31}=\int_{0}^{\pi} \mathrm{d} \theta \sin \theta \tau_{n} d_{k} x_{\theta} x^{p} \\
& L_{n k p}^{32}=\int_{0}^{\pi} \mathrm{d} \theta \sin \theta d_{n} d_{k} x^{p+1} .
\end{aligned}
$$

As for the spheroids, we can show that each of $L_{n k p}^{31}$ and $L_{n k p}^{32}$ is zero for the values of $p$ that we have seen make no contribution, except for the dominant term. In this case, it is the sum of the dominant term from each integral which is zero.

We consider first the dominant terms in the case where $p=k-n-1$. As for the 
spheroids, the ratio of these leading terms is

$$
\frac{\left.\chi_{n}^{\prime}\right|_{\text {leading term }}}{\left.\chi_{n}\right|_{\text {leading term }}}=-n
$$

From this, we may write

$$
\left.L_{n k p}^{3}\right|_{p=k-n-1}=\int_{0}^{\pi} \mathrm{d} \theta \sin \theta d_{k} n \gamma_{n k}(s)\left[x_{\theta} \tau_{n} x^{p}+(n+1) d_{n} x^{p+1}\right] .
$$

If $n+k$ is odd, then $p$ is even, and the parity of $\tau_{n} d_{k}$ is even by Eq. (E.16), and $d_{n} d_{k}$ is odd by Eq. (E.15), then we may substitute using the definitions of $e_{p}$ and $\tilde{u}_{p}$ to obtain

$$
\left.L_{n k p}^{3}\right|_{p=k-n-1}=\int_{0}^{\pi} \mathrm{d} \theta d_{k} n \gamma_{n k}(s)\left[\sin \theta \tau_{n} e_{p}+(n+1) \sin \theta d_{n} \tilde{u}_{p+1}\right] .
$$

It is possible to write the part in brackets by using Eqs. (B.15) and (E.44) as

$$
\sin \theta \tau_{n} e_{p}+(n+1) \sin \theta d_{n} \tilde{u}_{p+1}=\sqrt{(n+1)^{2}-m^{2}} d_{n+1} e_{p}+(n+1) u_{p+1} d_{n} /\left(k_{1} D\right)
$$

and thus the integral may be written

$$
\left.L_{n k p}^{3}\right|_{p=k-n-1}=\eta_{n k}(s) \int_{0}^{\pi} \mathrm{d} \theta e_{p} d_{k} d_{n+1}+\phi_{n k} \int_{0}^{\pi} \mathrm{d} \theta u_{p+1} d_{k} d_{n} .
$$

The first of these terms resembles $K_{n+1, k, p}^{1}$ (for the case where $p$ is even, as in Eq. (E.45), which is zero for $p=k-n+1$. The second term resembles $K_{n, k, p+1}^{1}$ (for the case where $p+1$ is odd, as Eq. (E.46)), and this is also zero when $p=k-n-1$.

We now turn to the case where $n+k$ is even, and so $p$ is odd. In this case, the parity of $\tau_{n} d_{k}$ is odd, and $d_{n} d_{k}$ is even, and so by considering this we end up with

$$
\left.L_{n k p}^{3}\right|_{p=k-n-1}=\int_{0}^{\pi} \mathrm{d} \theta d_{k} n \gamma_{n k}(s)\left[\sin \theta \tau_{n} u_{p}+(n+1) \sin \theta d_{n} \tilde{e}_{p+1}\right] .
$$


The term in brackets may now be written using Eqs. (B.15) and $($ E.43) as

$$
\sin \theta \tau_{n} u_{p}+(n+1) \sin \theta d_{n} \tilde{e}_{p+1}=\sqrt{(n+1)^{2}-m^{2}} d_{n+1} u_{p}+(n+1) e_{p+1} d_{n} /\left(k_{1} D\right)
$$

and hence the integral becomes

$$
\left.L_{n k p}^{3}\right|_{p=k-n-1}=\lambda_{n k}(s) \int_{0}^{\pi} \mathrm{d} \theta u_{p} d_{k} d_{n+1}+\zeta_{n k} \int_{0}^{\pi} \mathrm{d} \theta e_{p+1} d_{k} d_{n} .
$$

The first of these resembles Eq. (E.46), which is $K_{n+1, k, p}^{1}$ where $p$ is odd, and this is zero when $p=k-n-1$. The second term resembles Eq. (E.45), $K_{n, k, p+1}^{1}$ where $p+1$ is even, and this is also zero when $p=k-n-1$. Hence, the leading terms cancel out with each other, and make no contribution to the integral.

We can now consider the other terms. Looking at $L_{n k p}^{31}$, this has the same form as $L_{n k p}^{1}$, which is zero when $k-n+1 \leq p \leq n-k-3$, which is the rest of the range that we need to be zero. For $L_{n k p}^{32}$, more work is involved.

We start with the case where $n+k$ is odd, and so $p$ is even. Using the parity properties of the angular functions, we may then write

$$
L_{n k p}^{32}=\int_{0}^{\pi} \mathrm{d} \theta \sin \theta d_{n} d_{k} \tilde{u}_{p+1}
$$

Using Eq. E.42, this may be written (note that $p+1$ here is odd)

$$
L_{n k p}^{32}=\int_{0}^{\pi} \mathrm{d} \theta \sin \theta d_{n} d_{k} \mathcal{P}_{|p+1|}(\cos \theta)
$$

and then by Eq. (5.19) this is zero when $|p+1| \leq n-k-1$, which covers all of the required terms (except the already-accounted for dominant term).

In the case where $n+k$ is even (and $p$ is odd), then by parity we may write

$$
L_{n k p}^{32}=\int_{0}^{\pi} \mathrm{d} \theta \sin \theta d_{n} d_{k} \tilde{e}_{p+1}
$$


E Proof that Leading Terms Make No Contribution in Offset-Sphere Integrals

This may be rewritten using Eq. (E.41) (as $p+1$ is even) to

$$
L_{n k p}^{32}=\int_{0}^{\pi} \mathrm{d} \theta \sin \theta d_{n} d_{k} \mathcal{P}_{|p+1|}(\cos \theta)
$$

and is also zero by Eq. (5.19) when $|p+1| \leq n-k-1$, which is exactly the same condition as for $n+k$ odd. Thus, combined with the result for the dominant terms, all of the required integrals are identically zero.

The last integral is $L_{n k}^{4}$. However, this may be constructed as a linear combination of $L^{1}, L^{2}$ and $L^{3}$, and so a proof here is unnecessary. This completes the proofs for all of the integrals. 


\section{F Demonstration of Problems Near $s=1$}

As mentioned in Section 5.6.2, when computing the function $F_{n k}^{+}=\mathcal{P}^{+}\left(x \chi_{n}(x) \psi_{k}(s x)\right)$, there can be numerical problems when $s$ is close to one. The final method proposed in Section 5.6.2 does not suffer from these problems, but for completeness, a demonstration of the problems is provided here. This follows Appendix $\mathrm{C}$ of [6], where this was first presented.

We here take $n+k$ to be even, with $n \geq k+4$, as these are the terms that exhibit cancellations. We also are working with $s=1$, as that is where the problem manifests. Thus, we are working with

$$
F_{n k}^{+}(s=1, x)=\mathcal{P}^{+}\left(x \chi_{n}(x) \psi_{k}(x)\right)
$$

In the following, we omit the argument $x$ from all functions, and it should be taken as implied.

We begin by defining

$$
\Delta_{n k}=\psi_{n} \chi_{k}-\chi_{n} \psi_{k}
$$

Using this, we will show that $\mathcal{P}^{+}\left(x \chi_{n} \psi_{k}\right)$ contains only powers of $x$ starting at $n+1-k$. A similar equation was written by Waterman as Eq. (28a) in Ref. [72].

Trivially, we note that

$$
\Delta_{n n}=0
$$




\section{F Demonstration of Problems Near $s=1$}

and we may use the Wronskian relation (from Eq. (10.1.31) of Ref. [50]) to see that

$$
\Delta_{n+1, n}=\psi_{n+1} \chi_{n}-\chi_{n+1} \psi_{n}=1
$$

Following on from this, we define

$$
\nu_{p n}=x \Delta_{p+n, n},
$$

and look to find a recursion relation in $p$, for a given $n$. The initial conditions are

$$
\begin{aligned}
& \nu_{0, n}=x \Delta_{n n}=0 \\
& \nu_{1, n}=x \Delta_{n+1, n}=1 .
\end{aligned}
$$

We also note some relations on the Riccati-Bessel functions, namely

$$
\begin{aligned}
\psi_{n+1} \chi_{k}+\psi_{n-1} \chi_{k} & =\frac{2 n+1}{x} \psi_{n} \chi_{k} \\
\chi_{n+1} \psi_{k}+\chi_{n-1} \psi_{k} & =\frac{2 n+1}{x} \chi_{n} \psi_{k}
\end{aligned}
$$

and hence

$$
\Delta_{n+1, k}+\Delta_{n-k, 1}=\frac{2 n+1}{x} \Delta_{n k}
$$

Phrasing this in terms of $\nu_{p n}$, we have

$$
\nu_{p+1, n}=\frac{2 n+2 p+1}{x} \nu_{p n}-\nu_{p-1, n}
$$

From this, we may obtain, by using the initial conditions listed before, for the positive part of $\nu$

$$
\begin{aligned}
\nu_{2 q-1, n}^{+} & =(-1)^{q+1} x \\
\nu_{2 q, n}^{+} & =(-1)^{q+1} q(2 n+2 q+1) .
\end{aligned}
$$


Now, with $n+k$ even, we may write

$$
\left(x \Delta_{n k}\right)^{+}=\nu_{n-k, k}^{+}=(-1)^{(n-k+2) / 2} \frac{n-k}{2}(k+n+1) .
$$

Note also that as we are working with $n \geq k+4$, then $x \chi_{k} \psi_{n}=\left(x \chi_{k} \psi_{n}\right)^{+}$(beware of the swapped order of indices here, this means we are looking above the diagonal of our matrix, where there are no cancellations). From this, we may obtain

$$
x \chi_{n} \psi_{k}=x \chi_{k} \psi_{n}-x \Delta_{n k}=x \chi_{k} \psi_{n}+(-1)^{(n-k) / 2} \frac{n-k}{2}(n+k+1) .
$$

The significance of this is that the first term in the power series expansion of this is $x^{0}$, but the second term is $x^{n-k+1}$. This large gap leads to a lot of coefficients $\gamma_{q n k}$ (from Eq. (5.89) ) that should be identically zero (in the case of $s=1$ ), but instead are affected by numerical errors in the cancellation, and hence the calculated value of $F^{+}$is wrong in this case. 



\section{Bibliography}

[1] W. R. C. Somerville, B. Auguié, and E. C. Le Ru. Simplified expressions of the T-matrix integrals for electromagnetic scattering. Opt. Lett., 36(17):34823484, 2011.

[2] E. C. Le Ru, J. Grand, I. Sow, W. R. C. Somerville, P. G. Etchegoin, M. Treguer-Delapierre, G. Charron, N. Félidj, G. Lévi, and J. Aubard. A scheme for detecting every single target molecule with surface-enhanced Raman spectroscopy. Nano Lett., 11(11):5013-5019, 2011.

[3] W. R. C. Somerville, B. Auguié, and E. C. Le Ru. Severe loss of precision in calculations of T-matrix integrals. J. Quant. Spectrosc. Rad. Transfer, 113(7):524-535, 2012.

[4] W. R. C. Somerville, B. Auguié, and E. C. Le Ru. Distribution of the SERS enhancement factor on the surface of metallic nano-particles. In Nanotechnology (IEEE-NANO), 2012 12th IEEE Conference on, pages 1-4, 2012.

[5] R. Gill, L. Tian, W. R. C. Somerville, E. C. Le Ru, H. van Amerongen, and V. Subramaniam. Silver nanoparticle aggregates as highly efficient plasmonic antennas for fluorescence enhancement. J. Phys. Chem. C, 116(31):1668716693, 2012.

[6] W. R. C. Somerville, B. Auguié, and E. C. Le Ru. A new numerically stable implementation of the $T$-matrix method for electromagnetic scattering by spheroidal particles. J. Quant. Spectrosc. Rad. Transfer, 123:153-168, 2013.

[7] E. C. Le Ru, W. R. C. Somerville, and B. Auguié. Radiative correction in 


\section{Bibliography}

approximate treatments of electromagnetic scattering by point and body scatterers. Phys. Rev. A, 87(1):012504, 2013.

[8] C. F. Bohren and D. R. Huffman. Absorption and scattering of light by small particles. John Wiley \& Sons Inc., New York, 1983.

[9] M. I. Mishchenko, L. D. Travis, and A. A. Lacis. Scattering, absorption and emission of light by small particles. Cambridge University Press, Cambridge, 3rd electronic release edition, 2002.

[10] E. C. Le Ru and P. G. Etchegoin. Principles of surface-enhanced Raman spectroscopy and related plasmonic effects. Elsevier, Amsterdam, 2009.

[11] J. E. Hansen and J. B. Pollack. Near-infrared light scattering by terrestrial clouds. J. Atmos. Sci., 27(2):265-281, 1970.

[12] P. Yang and K. N. Liou. Finite-difference time domain method for light scattering by small ice crystals in three-dimensional space. J. Opt. Soc. Am. A, 13(10):2072-2085, 1996.

[13] I. Mann, H. Kimura, and L. Kolokolova. A comprehensive model to describe light scattering properties of cometary dust. J. Quant. Spectrosc. Rad. Transfer, 89(1):291-301, 2004.

[14] X. Shen, Q. Qin, Y. Hong, and G. Zhang. A matrix inversion approach of computing T-matrix for axially symmetrical particles of extreme shape and dielectrically large dimension. Radio Sci., 47(5):RS5005, 2012.

[15] T. Wessapan, S. Srisawatdhisukul, and P. Rattanadecho. Specific absorption rate and temperature distributions in human head subjected to mobile phone radiation at different frequencies. Int. J. Heat Mass Transfer, 55(1):347-359, 2012.

[16] A. M. K. Nilsson, P. Alsholm, A. Karlsson, and S. Andersson-Engels. T-matrix computations of light scattering by red blood cells. Appl. Optics, 37(13):2735$2748,1998$. 
[17] E. C. Le Ru, E. Blackie, M. Meyer, and P. G. Etchegoin. Surface enhanced Raman scattering enhancement factors: a comprehensive study. J. Phys. Chem. C, 111:13794-13803, 2007.

[18] E. Fort and S. Grésillon. Surface enhanced fluorescence. J. Phys. D: Appl. Phys., 41(1):013001, 2008.

[19] J. D. Jackson. Classical Electrodynamics. Wiley, New York, 2nd edition, 1975.

[20] S. Y. Matrosov. Modeling backscatter properties of snowfall at millimeter wavelengths. J. Atmos. Sci., 64(5):1727-1736, 2007.

[21] D. A. Long. The Raman effect: A unified treatment of the theory of Raman scattering by molecules. John Wiley \& Sons Ltd, Chichester, 2002.

[22] M. Fleischmann, P. J. Hendra, and A. J. McQuillan. Raman spectra of pyridine adsorbed at a silver electrode. Chem. Phys. Lett., 26(2):163-166, 1974.

[23] P. G. Etchegoin and E. C. Le Ru. Single-molecule SERS. Annu. Rev. Phys. Chem., 63(1):65-87, 2012.

[24] S. Nie and S. R. Emory. Probing single molecules and single nanoparticles by surface-enhanced Raman scattering. Science, 275:1102-1106, 1997.

[25] K. Kneipp, Y. Wang, H. Kneipp, L. T. Perelman, I. Itzkan, R. R. Dasari, and M. S. Feld. Single molecule detection using surface-enhanced Raman scattering (SERS). Phys. Rev. Lett., 78:1667-1670, 1997.

[26] E. C. Le Ru, M. Meyer, and P. G. Etchegoin. Proof of single-molecule sensitivity in surface enhanced Raman scattering (SERS) by means of a two-analyte technique. J. Phys. Chem. B, 110:1944-1948, 2006.

[27] E. C. Le Ru, S. A. Meyer, C. Artur, P. G. Etchegoin, J. Grand, P. Lang, and F. Maurel. Experimental demonstration of surface selection rules for SERS on flat metallic surfaces. Chem. Commum., 47:3903-3905, 2011.

[28] C. Artur, E. C. Le Ru, and P. G. Etchegoin. Temperature dependence of the homogeneous broadening of resonant Raman peaks measured by 


\section{Bibliography}

single-molecule surface-enhanced Raman spectroscopy. J. Phys. Chem. Lett., 2(23):3002-3005, 2011.

[29] W. E. Doering and S. M. Nie. Single-molecule and single nanoparticle SERS: Examining the roles of surface active sites and chemical enhancement. J. Phys. Chem. B, 106:311-317, 2002.

[30] A. Otto, A. Bruckbauer, and Y. X. Chen. On the chloride activation in SERS and single molecule SERS. J. Mol. Struct., 661:501-514, 2003.

[31] M. Moskovits. Surface roughness and the enhanced intensity of Raman scattering by molecules adsorbed on metals. J. Chem. Phys., 69:4159-4161, 1978.

[32] E. C. Le Ru and P. G. Etchegoin. Rigorous justification of the $|E|^{4}$ enhancement factor in surface enhanced Raman spectroscopy. Chem. Phys. Lett., 423(1):63-66, 2006.

[33] F. M. Kahnert. Numerical methods in electromagnetic scattering theory. $J$. Quant. Spectrosc. Rad. Transfer, 79-80:775-824, 2003.

[34] G. Mie. Beiträge zur optik trüber medien, speziell kolloidaler metallösungen. Ann. Phys., 25(3):377-445, 1908.

[35] D. W. Mackowski. Discrete dipole moment method for calculation of the $T$ matrix for nonspherical particles. J. Opt. Soc. Am. A, 19(5):881-893, 2002.

[36] T. A. Nieminen, H. Rubinsztein-Dunlop, and N. R. Heckenberg. Calculation of the $T$-matrix: general considerations and application of the point-matching method. J. Quant. Spectrosc. Rad. Transfer, 79:1019-1029, 2003.

[37] F. M. Kahnert, J. J. Stamnes, and K. Stamnes. Application of the extended boundary condition method to homogeneous particles with point-group symmetries. Appl. Optics, 40(18):3110-3123, 2001.

[38] COMSOL AB. http://www. comsol.com, 2011.

[39] K. Yee. Numerical solution of initial boundary value problems involving Maxwell's equations in isotropic media. IEEE Trans. Antennas Propagat., 14(3):302-307, 1966. 
[40] E. M. Purcell and C. R. Pennypacker. Scattering and absorption of light by nonspherical dielectric grains. Astrophys. J., 186:705-714, 1973.

[41] B. T. Draine and P. J. Flatau. Discrete-dipole approximation for scattering calculations. J. Opt. Soc. Am. A, 11(4):1491-1499, 1994.

[42] W.-H. Yang, G. C. Schatz, and R. P. Van Duyne. Discrete dipole approximation for calculating extinction and Raman intensities for small particles with arbitrary shapes. J. Chem. Phys., 103(3):869-875, 1995.

[43] P. J. Flatau and B. T. Draine. Fast near field calculations in the discrete dipole approximation for regular rectilinear grids. Optics Express, 20(2):1247-1252, 2012.

[44] A. M. Kern and O. J. F. Martin. Surface integral formulation for 3D simulations of plasmonic and high permittivity nanostructures. J. Opt. Soc. Am. A, 26(4):732-740, 2009.

[45] B. Gallinet, A. M. Kern, and O. J. F. Martin. Accurate and versatile modeling of electromagnetic scattering on periodic nanostructures with a surface integral approach. J. Opt. Soc. Am. A, 27(10):2261-2271, 2010.

[46] A. M. Kern and O. J. F. Martin. Excitation and reemission of molecules near realistic plasmonic nanostructures. Nano Lett., 11(2):482-487, 2011.

[47] P. C. Waterman. Symmetry, unitarity, and geometry in electromagnetic scattering. Phys. Rev. D, 3:825-839, 1971.

[48] P. C. Waterman. New formulation of acoustic scattering. J. Acoust. Soc. Am, 45(6):1417-1429, 1969.

[49] P. C. Waterman. Matrix formulation of electromagnetic scattering. Proc. IEEE, 53:805-812, 1965.

[50] M. Abramowitz and I. A. Stegun, editors. Handbook of Mathematical Functions. Dover, New York, 1972. 


\section{Bibliography}

[51] P. Barber and C. Yeh. Scattering of electromagnetic waves by arbitrarily shaped dielectric bodies. Appl. Optics, 14(12):2864-2872, 1975.

[52] A. Lakhtakia, M. F. Iskander, and C. H. Durney. An iterative extended boundary condition method for solving the absorption characteristics of lossy dielectric objects of large aspect ratios. IEEE Trans. Microwave Theory Tech., 31(8):640-647, 1983.

[53] A. Doicu and T. Wriedt. Calculation of the T matrix in the null-field method with discrete sources. J. Opt. Soc. Am. A, 16(10):2539-2544, 1999.

[54] V. L. Y. Loke, T. A. Nieminen, N. R. Heckenberg, and H. Rubinsztein-Dunlop. T-matrix calculation via discrete dipole approximation, point matching and exploiting symmetry. J. Quant. Spectrosc. Rad. Transfer, 110(14):1460-1471, 2009.

[55] V. L. Y. Loke, T. A. Nieminen, S. J. Parkin, N. R. Heckenberg, and H. Rubinsztein-Dunlop. FDFD/T-matrix hybrid method. J. Quant. Spectrosc. Rad. Transfer, 106(1-3):274-284, 2007.

[56] B. Peterson and S. Ström. T-matrix formulation of electromagnetic scattering from multilayered scatterers. Phys. Rev. D, 10:2670-2684, 1974.

[57] B. Peterson and S. Ström. T matrix for electromagnetic scattering from an arbitrary number of scatterers and representations of $\mathrm{E}(3)$. Phys. Rev. D, 8(10):3661-3678, 1973.

[58] A. Doicu, T. Wriedt, and Y. A. Eremin. Light scattering by systems of particles. Springer, Berlin, 2006.

[59] P. C. Waterman and R. Truell. Multiple scattering of waves. J. Math. Phys., 2(4):512-537, 1961.

[60] M. I. Mishchenko. Light scattering by randomly oriented axially symmetric particles. J. Opt. Soc. Am. A, 8(6):871-882, 1991. 
[61] M. I. Mishchenko. Light scattering by size-shape distributions of randomly oriented axially symmetric particles of a size comparable to a wavelength. Appl. Optics, 32(24):4652-4666, 1993.

[62] W. Vargas, L. Cruz, L. F. Fonseca, and M. Gómez. T-matrix approach for calculating local fields around clusters of rotated spheroids. Appl. Optics, 32(12):2164-2170, 1993.

[63] A. Moroz. Improvement of Mishchenko's T-matrix code for absorbing particles. Appl. Optics, 44(17):3604-3609, 2005.

[64] M. I. Mishchenko and L. D. Travis. T-matrix computations of light scattering by large spheroidal particles. Opt. Commun., 109(1-2):16-21, 1994.

[65] D. J. Wielaard, M. I. Mishchenko, A. Macke, and B. E. Carlson. Improved Tmatrix computations for large, nonabsorbing and weakly absorbing nonspherical particles and comparison with geometrical-optics approximation. Appl. Optics, 36(18):4305-4313, 1997.

[66] R. F. Millar. Rayleigh hypothesis in scattering problems. Electron. Lett., 5(17):416-417, 1969.

[67] V. Farafonov. Applicability of the $T$-matrix method and its modifications. Opt. Spectrosc., 92:748-760, 2002.

[68] A. Doicu and T. Wriedt. Near-field computation using the null-field method. J. Quant. Spectrosc. Rad. Transfer, 111(3):466-473, 2010.

[69] C. Forestiere, G. Iadarola, L. Dal Negro, and G. Miano. Near-field calculation based on the T-matrix method with discrete sources. J. Quant. Spectrosc. Rad. Transfer, 112(14):2384-2394, 2011.

[70] A. Doicu and T. Wriedt. Extended boundary condition method with multipole sources located in the complex plane. Opt. Commun., 139(1-3):85-91, 1997.

[71] T. Wriedt and A. Doicu. Formulations of the extended boundary condition method for three-dimensional scattering using the method of discrete sources. J. Mod. Optic., 45(1):199-213, 1998. 


\section{Bibliography}

[72] P. C. Waterman. The T-matrix revisited. J. Opt. Soc. Am. A, 24(8):2257$2267,2007$.

[73] P. C. Waterman. T-matrix methods in acoustic scattering. J. Acoust. Soc. Am, 125(1):42-51, 2009.

[74] GMP, the GNU Multiple Precision Arithmetic Library. http://gmplib.org/, 2010.

[75] L. Fousse, G. Hanrot, V. Lefèvre, P. Pélissier, and P. Zimmermann. MPFR: A multiple-precision binary floating-point library with correct rounding. $A C M$ Trans. Math. Software, 33(2):13:1-13:15, 2007.

[76] MPC, version 0.9. http://multiprecision.org/, 2011.

[77] M. Nakata. The MPACK (MBLAS/MLAPACK); a multiple precision arithmetic version of BLAS and LAPACK, version 0.6.7. http://mplapack. sourceforge.net/, 2010.

[78] J. A. Gaunt. The triplets of helium. Philos. Trans. R. Soc. Lond., A, 228:151$196,1929$.

[79] D. Petrov, Y. Shkuratov, and G. Videen. Optimized matrix inversion technique for the T-matrix method. Opt. Lett., 32:1168-1170, 2007.

[80] V. Strassen. Gaussian elimination is not optimal. Numer. Math., 13:354-356, 1969.

[81] L. Li. Comment on "Optimized matrix inversion technique for the T-matrix method". Opt. Lett., 33(12):1366, 2008.

[82] Y. Fang, N.-H. Seong, and D. D. Dlott. Measurement of the distribution of site enhancements in surface-enhanced Raman scattering. Science, 321:388-391, 2008.

[83] P. G. Etchegoin, E. C. Le Ru, and M. Meyer. An analytic model for the optical properties of gold. J. Chem. Phys., 125:164705, 2006. 
[84] E. C. Le Ru, M. Meyer, E. Blackie, and P. G. Etchegoin. Advanced aspects of electromagnetic SERS enhancement factors at a hot-spot. J. Raman Spectrosc., 39:1127-1134, 2008.

[85] E. C. Le Ru, E. Blackie, M. Meyer, and P. G. Etchegoin. Surface enhanced Raman scattering enhancement factors: a comprehensive study. J. Phys. Chem. C, 111:13794-13803, 2007.

[86] P. D. Maker, R. W. Terhune, M. Nisenoff, and C. M. Savage. Effects of dispersion and focusing on the production of optical harmonics. Phys. Rev. Lett., 8(1):21-22, 1962.

[87] S. B. Chaney, S. Shanmukh, R. A. Dluhy, and Y.-P. Zhao. Aligned silver nanorod arrays produce high sensitivity surface-enhanced Raman spectroscopy substrates. Appl. Phys. Lett., 87(3):031908, 2005.

[88] R. Gupta and W. A. Weimer. High enhancement factor gold films for surface enhanced Raman spectroscopy. Chem. Phys. Lett., 374(3):302-306, 2003.

[89] L. Rivas, S. Sanchez-Cortes, J. V. Garcia-Ramos, and G. Morcillo. Growth of silver colloidal particles obtained by citrate reduction to increase the Raman enhancement factor. Langmuir, 17(3):574-577, 2001.

[90] J. Fang, Y. Yi, B. Ding, and X. Song. A route to increase the enhancement factor of surface enhanced Raman scattering (SERS) via a high density Ag flower-like pattern. Appl. Phys. Lett., 92(13):131115, 2008.

[91] K. Kneipp, H. Kneipp, G. Deinum, I. Itzkan, R. R. Dasari, and M. S. Feld. Single-molecule detection of cyanine dye in silver colloidal solution using nearinfrared surface-enhanced Raman scattering. Appl. Spectrosc., 52:175-178, 1998.

[92] R. Boyack and E. C. Le Ru. Investigation of particle shape and size effects in SERS using T-matrix calculations. Phys. Chem. Chem. Phys., 11:7398-7405, 2009. 


\section{Bibliography}

[93] A. G. Brolo, D. E. Irish, and B. D. Smith. Applications of surface enhanced Raman scattering to the study of metal-adsorbate interactions. J. Mol. Struct., 405(1):29-44, 1997.

[94] M. D. Porter, R. J. Lipert, L. M. Siperko, G. Wang, and R. Narayanan. SERS as a bioassay platform: fundamentals, design, and applications. Chem. Soc. Rev., 37(5):1001-1011, 2008.

[95] S. Shanmukh, L. Jones, J. Driskell, Y. Zhao, R. Dluhy, and R. A. Tripp. Rapid and sensitive detection of respiratory virus molecular signatures using a silver nanorod array SERS substrate. Nano Lett., 6(11):2630-2636, 2006.

[96] F. Inscore, C. Shende, A. Sengupta, H. Huang, and S. Farquharson. Detection of drugs of abuse in saliva by surface-enhanced Raman spectroscopy (SERS). Appl. Spectrosc., 65(9):1004-1008, 2011.

[97] M. Sanles-Sobrido, L. Rodríguez-Lorenzo, S. Lorenzo-Abalde, Á. GonzálezFernández, M. A. Correa-Duarte, R. A. Alvarez-Puebla, and L. M. Liz-Marzán. Label-free SERS detection of relevant bioanalytes on silver-coated carbon nanotubes: the case of cocaine. Nanoscale, 1(1):153-158, 2009.

[98] S. Botti, L. Cantarini, and A. Palucci. Surface-enhanced Raman spectroscopy for trace-level detection of explosives. J. Raman Spectrosc., 41(8):866-869, 2010.

[99] A. Chou, E. Jaatinen, R. Buividas, G. Seniutinas, S. Juodkazis, E. L. Izake, and P. M. Fredericks. SERS substrate for detection of explosives. Nanoscale, $4(23): 7419-7424,2012$.

[100] H. Wackerbarth, C. Salb, L. Gundrum, M. Niederkrüger, K. Christou, V. Beushausen, and W. Viöl. Detection of explosives based on surfaceenhanced Raman spectrosscopy. Appl. Optics, 49(23):4362-4366, 2010. 